\title{
Abstracts of the XXXVIII Congress of the Spanish Society of Physiological Sciences (SECF), 13-16 September 2016, Zaragoza, Spain
}

\section{Dear colleagues,}

For the Organising Committee is an honour to host the XXXVIII Congress of the Spanish Society of Physiological Sciences (SECF) in Zaragoza. We are proud to welcome all of the participants to our city during this event that will take place between 13th and 16th September 2016.

Throughout the different meetings, the SECF Congress has been considered an important instrument in attaining one of the main objectives of the Society, which is "to generate interest, stimulate research and disseminate knowledge of Physiology and its applications". We hope that this Congress, like all the previous ones, will offer us the opportunity to learn all about the current research activities of our colleagues in the field of physiology, encouraging contact between scientists and facilitating the exchange of information.

Different studies will be presented in the form of plenary sessions, symposia and posters and will include superb scientific material that was carefully selected by the Scientific Committee. These studies, originating from laboratories from all over Spain, assure that the meeting will be a major scientific event. Topics cover a wide range of subjects, mainly related to Cell and Molecular Physiology, Neurophysiology, Endocrinology, Cardiovascular Physiology, Respiratory Physiology, Gastrointestinal Physiology, Renal Physiology, Nutrition, metabolism and obesity, Chronobiology, Bioengineering, Teaching of Physiology and Sports Physiology.

As President of the Organising Committee, I greatly appreciate the effort of the contributing authors, and especially of the invited speakers, the chairpersons and colleagues from the Organising and Scientific Committees. Last but not least, I would like to express my gratitude to the commercial and institutional sponsors, without whose support it would have been impossible to organise this Congress.

We hope this meeting will be fruitful, not only from the scientific point of view but also as a social event. Enjoy the hospitality of Zaragoza, its weather, the taste of its gastronomy and its rich history.

Ignacio Giménez President of the Organising Committee 


\section{Meeting of the Spanish Society for Physiological Sciences}

Zaragoza, September 13-16, 2016

\begin{tabular}{|c|c|c|c|c|}
\hline \multicolumn{5}{|c|}{ Tuesday, September 13, 2016} \\
\hline Hour/Room & Aula Magna & Pilar Sinués & Joaquín Costa & Exhibition room \\
\hline $15: 30$ & \multicolumn{4}{|c|}{ Secretary/Registry opens } \\
\hline $16: 00$ & \multirow{3}{*}{$\begin{array}{l}\text { S1-Applications of emerging } \\
\text { technologies in Physiology }\end{array}$} & & \multirow{3}{*}{$\begin{array}{l}\text { S2- Homeostatic deviations } \\
\text { in rare diseases }\end{array}$} & \\
\hline $16: 30$ & & & & \\
\hline $17: 00$ & & & & \\
\hline $17: 30$ & \multirow{4}{*}{\multicolumn{4}{|c|}{$\begin{array}{l}\text { Opening Session. L1-Challenges for Physiology in a technological future. Micro/nano technologies to fuel } \\
\text { Physiological research. Dra. Rosa Villa. Centro Nacional de Microelectrónica de Barcelona. }\end{array}$}} \\
\hline $18: 00$ & & & & \\
\hline $18: 30$ & & & & \\
\hline 19:00 & & & & \\
\hline $19: 30$ & \multirow{2}{*}{\multicolumn{4}{|c|}{ Welcome Reception }} \\
\hline $20: 00$ & & & & \\
\hline
\end{tabular}

\begin{tabular}{|c|c|c|c|c|}
\hline \multicolumn{5}{|c|}{ Wednesday, September 14, 2016} \\
\hline Hora/Sala & Sala Aula Magna & Sala Pilar Sinués & Sala Joaquín Costa & Sala Pósters (JAB) \\
\hline 9:00 & \multirow{4}{*}{$\begin{array}{l}\text { S3- Epithelial Transport. } \\
\text { Symposium homage to } \\
\text { Professor Anabel Alcalde. }\end{array}$} & \multirow{4}{*}{$\begin{array}{l}\text { S4-Cardiovascular } \\
\text { adaptations to long term and } \\
\text { high intensity exercise: } \\
\text { physiological or } \\
\text { pathological? }\end{array}$} & \multirow{4}{*}{$\begin{array}{l}\text { Oral Session 1: } \\
\text { Cell \& Molecular Physiology }\end{array}$} & \multirow{4}{*}{ Physiology Quiz - 1st Round } \\
\hline 9:30 & & & & \\
\hline $10: 00$ & & & & \\
\hline $10: 30$ & & & & \\
\hline $11: 00$ & \multirow{2}{*}{\multicolumn{4}{|c|}{$\begin{array}{c}\text { Coffe Break/Exhibitions } \\
\text { Poster Sessions: Cell \& Molecular Physiology, Neurophysiology, Cardiovascular, Gastrointestinal, Endocrinology, } \\
\text { Teaching. }\end{array}$}} \\
\hline $11: 30$ & & & & \\
\hline $12: 00$ & \multirow{4}{*}{\multicolumn{4}{|c|}{$\begin{array}{l}\text { SECF Annual Meeting. } \\
\text { Roberto Gallego Teaching in Physiology Award }\end{array}$}} \\
\hline $12: 30$ & & & & \\
\hline 13:00 & & & & \\
\hline 13:30 & & & & \\
\hline $14: 00$ & \multirow{2}{*}{\multicolumn{4}{|c|}{ Lunch Break }} \\
\hline $14: 30$ & & & & \\
\hline $15: 00$ & \multirow{2}{*}{\multicolumn{4}{|c|}{ Physiology Trainee Workshop }} \\
\hline $15: 30$ & & & & \\
\hline $16: 00$ & \multirow{4}{*}{$\begin{array}{l}\text { S6- New insights on the role } \\
\text { of ion channels in health and } \\
\text { disease }\end{array}$} & \multirow{4}{*}{$\begin{array}{l}\text { S5- A new role for the } \\
\text { carotid body in Pathology }\end{array}$} & \multirow{4}{*}{$\begin{array}{l}\text { Oral Session 2: } \\
\text { Gastrointestinal }\end{array}$} & \\
\hline $16: 30$ & & & & \\
\hline $17: 00$ & & & & \\
\hline $17: 30$ & & & & \\
\hline $18: 00$ & \multicolumn{4}{|c|}{ Break } \\
\hline $18: 30$ & \multirow{3}{*}{\multicolumn{4}{|c|}{$\begin{array}{l}\text { L2- Advances in Cell and Molecular Physiology Lecture. Optoelectrical dynamics of ion channels in } \\
\text { subcellular calcium nanodomains Teresa Giraldez, Universidad de La Laguna. }\end{array}$}} \\
\hline 19:00 & & & & \\
\hline 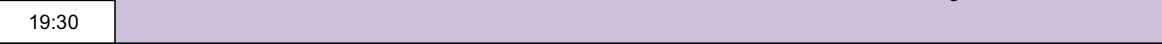 & & & & \\
\hline \multicolumn{5}{|l|}{ 20:00 } \\
\hline \multicolumn{5}{|l|}{$20: 30$} \\
\hline 21:00 & \multirow{2}{*}{\multicolumn{4}{|c|}{ Social Program (optional): Guided Tour to Aljaferia Palace }} \\
\hline $21: 30$ & & & & \\
\hline $22: 00$ & \multirow{2}{*}{\multicolumn{4}{|c|}{ Social Program (optional): Casual dinner (tapas in historic downtown) }} \\
\hline $22: 30$ & & & & \\
\hline
\end{tabular}




\begin{tabular}{|c|c|c|c|c|}
\hline \multicolumn{5}{|c|}{ Thursday, September 15, 2016} \\
\hline Hora/Sala & Sala Aula Magna & Sala Pilar Sinués & Sala Joaquín Costa & Sala Pósters (JAB) \\
\hline 9:00 & \multirow{4}{*}{$\begin{array}{l}\text { Oral Session 3: } \\
\text { Sports Physiology }\end{array}$} & \multirow{4}{*}{$\begin{array}{l}\text { Oral Session 4: } \\
\text { Neurophysiology }\end{array}$} & \multirow{4}{*}{$\begin{array}{l}\text { Oral Session 5: CV, } \\
\text { Respiratory, Teaching }\end{array}$} & \multirow{4}{*}{$\begin{array}{l}\text { Physiology Quiz - 2nd } \\
\text { Round }\end{array}$} \\
\hline 9:30 & & & & \\
\hline $10: 00$ & & & & \\
\hline $10: 30$ & & & & \\
\hline $11: 00$ & \multirow{2}{*}{\multicolumn{4}{|c|}{$\begin{array}{c}\text { Coffe Break/Exhibitions } \\
\text { Poster Session: Neurophysiology, Nutrition \& Metabolism, } \\
\text { Renal Respiratory, Sports Physiology, Chronobiology, Bioengineering }\end{array}$}} \\
\hline $11: 30$ & & & & \\
\hline $12: 00$ & \multirow{4}{*}{ S7- Teaching of Physiology. } & \multirow{4}{*}{ Round Table on Funding } & \multirow{4}{*}{$\begin{array}{l}\text { Oral Session 6: } \\
\text { Cardiovascular }\end{array}$} & \\
\hline $12: 30$ & & & & \\
\hline 13:00 & & & & \\
\hline \multicolumn{2}{|l|}{$13: 30$} & & & \\
\hline $14: 00$ & \multirow{2}{*}{\multicolumn{4}{|c|}{ Lunch Break }} \\
\hline $14: 30$ & & & & \\
\hline $15: 00$ & \multirow{2}{*}{\multicolumn{4}{|c|}{ Physiology Trainee Workshop }} \\
\hline $15: 30$ & & & & \\
\hline 16:00 & \multirow{4}{*}{$\begin{array}{l}\text { S8- Gastrointestinal } \\
\text { Physiology: functions of } \\
\text { intestinal mucose and } \\
\text { smooth muscle }\end{array}$} & \multirow{4}{*}{$\begin{array}{l}\text { S9- Biological rhythms, light } \\
\text { and health }\end{array}$} & \multirow{4}{*}{$\begin{array}{l}\text { S10-High throughput } \\
\text { technologies applied to } \\
\text { nutritional and } \\
\text { cardiovascular research }\end{array}$} & \\
\hline $16: 30$ & & & & \\
\hline $17: 00$ & & & & \\
\hline $17: 30$ & & & & \\
\hline 18:00 & \multicolumn{4}{|c|}{ Break } \\
\hline $18: 30$ & \multirow{3}{*}{\multicolumn{4}{|c|}{$\begin{array}{c}\text { L3-Advances in Applied Physiology. "Chronodisruption and melatonergic dysfunction in aging and disease". } \\
\text { Rudiger Hardeland, University of Goettingen, Germany }\end{array}$}} \\
\hline 19:00 & & & & \\
\hline ( & & & & \\
\hline \multicolumn{5}{|l|}{ 20:00 } \\
\hline \multicolumn{5}{|l|}{$20: 30$} \\
\hline 21:00 & \multirow{3}{*}{\multicolumn{4}{|c|}{ Social Program (optional): Conference Dinner }} \\
\hline $21: 30$ & & & & \\
\hline 22:00 & & & & \\
\hline
\end{tabular}

\begin{tabular}{|c|c|c|c|c|}
\hline \multicolumn{5}{|c|}{ Friday, September 16, 2016} \\
\hline Hora/Sala & Sala Aula Magna & Sala Pilar Sinués & Sala Joaquín Costa & Sala Pósters (JAB) \\
\hline $9: 00$ & \multirow{3}{*}{$\begin{array}{l}\text { Oral Session 7: } \\
\text { Endocrinology, } \\
\text { Chronobiology, Nutrition }\end{array}$} & & \multirow{3}{*}{$\begin{array}{c}\text { Oral Session 8: } \\
\text { Renal, Cell \& Mol Physiology }\end{array}$} & \\
\hline $9: 30$ & & & & \\
\hline $10: 00$ & & & & \\
\hline $10: 30$ & \multirow{2}{*}{\multicolumn{4}{|c|}{$\begin{array}{l}\text { Coffe Break/Exhibitions } \\
\text { Poster Session/Physiology Trainee Workshop: Cardiovascular, Cell \& Molecular Physiology }\end{array}$}} \\
\hline $11: 00$ & & & & \\
\hline $11: 30$ & \multirow{3}{*}{$\begin{array}{l}\text { S11-Vascular dysfunction, } \\
\text { obesity and insulin } \\
\text { resistance }\end{array}$} & & \multirow{3}{*}{$\begin{array}{l}\text { S12-Neuropathic pain: a } \\
\text { problem with difficult } \\
\text { diagnosis and therapy }\end{array}$} & \\
\hline $12: 00$ & & & & \\
\hline $12: 30$ & & & & \\
\hline $13: 00$ & \multirow{4}{*}{\multicolumn{4}{|c|}{$\begin{array}{l}\text { Closing ceremony. SECF's } 2016 \text { Juan Negrín Award Lecture An } \\
\text { unfolding connection between yoga and muscle contraction } \\
\text { Julio Fernández, Single Protein Mechanics and Engineering Laboratory } \\
\text { Columbia University, US }\end{array}$}} \\
\hline $13: 30$ & & & & \\
\hline $14: 00$ & & & & \\
\hline $14: 30$ & & & & \\
\hline
\end{tabular}




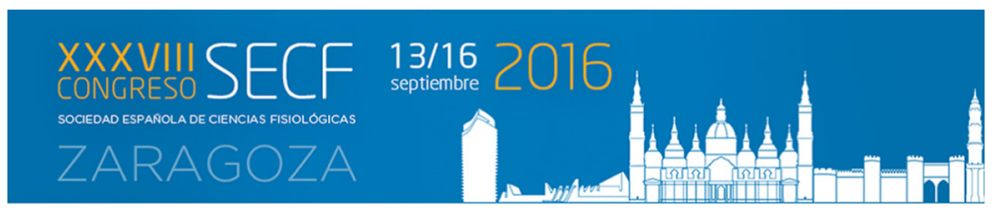

\section{Congress Committees}

ORGANIZING COMMITTEE

- Ignacio Giménez López

Universidad de Zaragoza

- Javier Miana Mena

Universidad de Zaragoza

- Lorena Fuentes Broto

Universidad de Zaragoza

- José Manuel Lou Bonafonte

Universidad de Zaragoza

- Guillermo Álvarez de Toledo Naranjo

SECF President, U. Sevilla

- Alfonso Mate Barrero

SECF Secretary, U. Sevilla

- Antonio Felipe Campo

SECF Treasurer, U. Barcelona

- Beatriz Puisac Uriol

Universidad de Zaragoza

- Laura Grasa López

Universidad de Zaragoza

- Marta Castro López

Universidad de Zaragoza

\author{
SCIENTIFIC COMITTEE \\ - Rüdiger Hardeland \\ Gottingen University, Germany \\ - Javier Cudeiro Mazaira \\ SECF Past President, U. de A Coruña \\ - Casto Rivadulla Fernández \\ SECF Vocal, U.d de A Coruña \\ - Paloma Alonso Magdalena \\ SECF Vocal, U. Miguel Hernández \\ - Emilio Martínez de Victoria \\ SECF Elect President, U. de Granada \\ - Jesús Escanero Marcén \\ Universidad de Zaragoza \\ - José Octavio Alda Torrubia \\ Universidad de Zaragoza \\ - Pilar Arruebo Loshuertos \\ Universidad de Zaragoza \\ - José Joaquín García García \\ Universidad de Zaragoza \\ - Ma Jesús Rodriguez Yoldi \\ Universidad de Zaragoza \\ - Juan Pie Juste \\ Universidad de Zaragoza \\ - José Emilio Mesonero Gutiérrez \\ Universidad de Zaragoza \\ - Miguel Ángel Plaza Carrión \\ Universidad de Zaragoza
}

\section{LOCAL COMITTEE}

- Manuel Guerra Sánchez

Universidad de Zaragoza

- Marisol Soria Aznar

Universidad de Zaragoza

Instituto Aragonés de Ciencias de la Salud

- Desireé Pereboom Maicas

Universidad de Zaragoza

- Laura Martínez Gimeno

Instituto Aragonés de Ciencias de la Salud
GRUPOPACIFICO

CONGRESS TECHNICAL SECRETARY
The power of meeting 


\section{Sponsors}
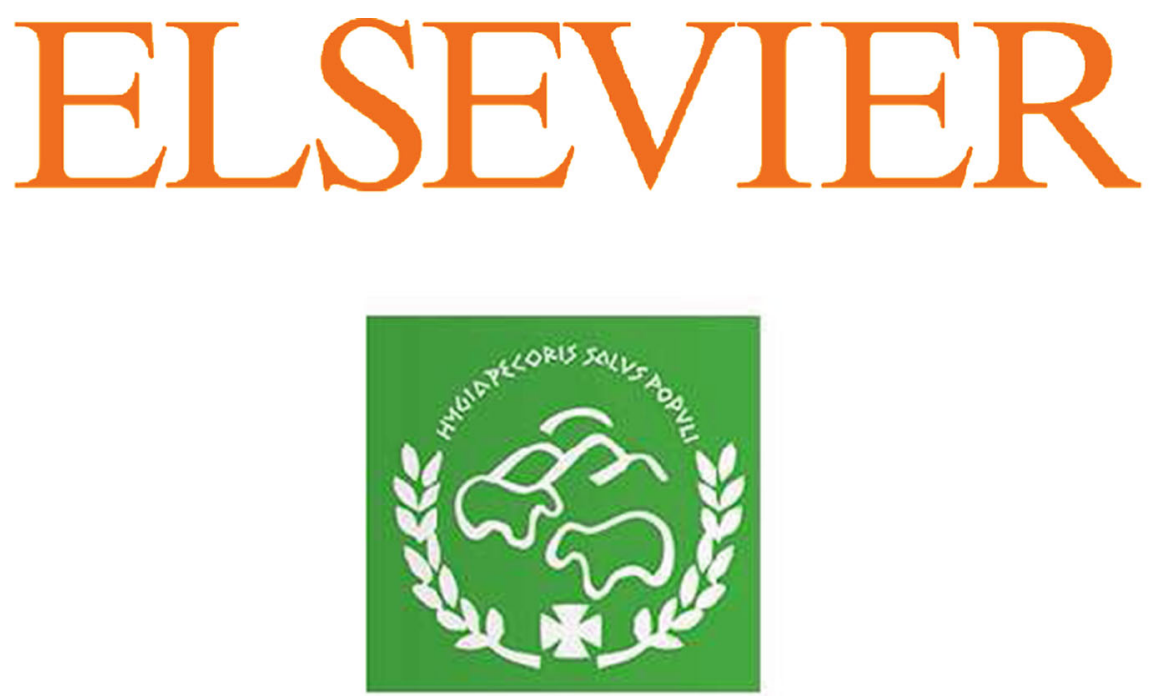

Consejo General de Colegios de Veterinarios

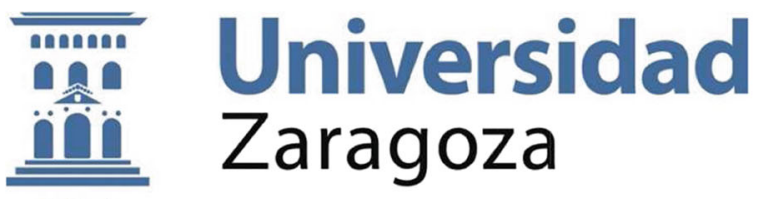

1542

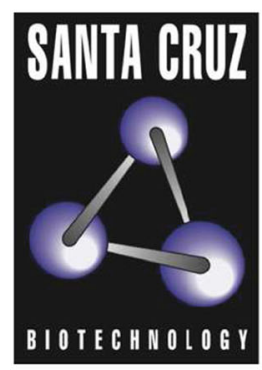

The Power to Question

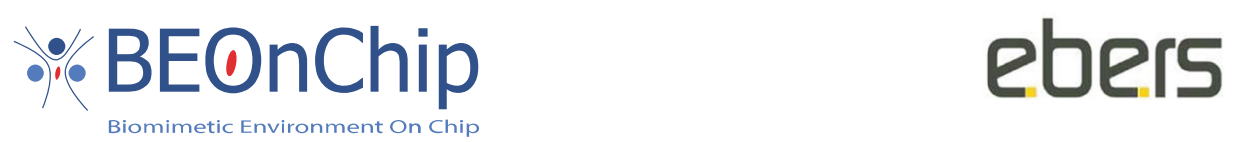




\section{KEYNOTE LECTURES}

L1

\section{MICRO/NANOTECHNOLOGIES TO FUEL PHYSIOLOGICAL RESEARCH}

\section{Rosa Villa Sanz}

Instituto de Microelectrónica de Barcelona, Bellaterra (Barcelona), Spain

Micro- and nanotechnologies are strongly associated with the design and manufacture of the integrated circuits that are present in the chips of our computer or cell phone, being the basis of the actual technological revolution. Besides being so common in the devices of our daily life, in the near future they are foreseen to interconnect many everyday objects in what is called the Internet of things.

These technologies are also an opportunity for many advances in medicine, not only for enhance the communications but to achieve smaller devices with many different features. Among the large amount of devices, it is noteworthy to highlight the pacemakers or cochlear implants.

Micro and nanotechnology fabrication processes have achieved astonishing advances in manufacturing chips. I will show that these technologies are more accessible than what is believed and its implementation is possible to produce innovative and disruptive devices that may have a great potential in the area of physiological sciences.

I will describe two examples in which we are working that combine the use of micro- and nanotechnology fabrication processes and the use of novel materials with the needs identified by physiologists. This strategy allows the creation of new tools for cellular and molecular science.

In particular, I will expose how a new material such as graphene can have a crucial paper on the future of neurosciences; and the advances in using new microdevices called "organ on a chip", which will play a crucial role for future studies in drug discovery and disease modeling and treatment.

Graphene is a 2D material formed by a monolayer of carbon atoms (discovered by A. Gueim and K. Novosiólov, Nobel prize in 2010) which has recently attracted a high interest by both the scientific community and the mass media due to its excellent properties. In fact, its transparency, flexibility and outstanding electrical properties are expected to revolutionize the electronics. European flagship projects such as the Graphens Flasgship are developing a large activity to implement graphene in different fields, including the biomedical. Our group is now working on the development of flexible neuroprobes with integrated graphene transistors that are capable to register multiple neural signals. Our preliminary results demonstrate the possibilities of these active devices which allow the possibility of multiplexing the signals; thus, increasing the density of the registers which will definitely increase the knowledge of the nervous system.

The second example is not directly based on the use of new material but in the ease of combining different micro- and nanotechnology processesto to develop different microstructures. The "organs on a chip" are microfluidic devices in which cells can be cultured in an environment that is engineered in such a way that it better replicates the in vivo microenvironment of an specific tissue. Our experience is related to the development of two devices: the Liver on chip and BBB (blood brain barrier) on Chip. The results show significant changes compared to the standard static cell cultures. In particular, cells show a much similar response to the physiological conditions. However, its ease of use is still one of the challenges for incorporating these devices in laboratories routinely.

New materials and new micro- and nanotechnology processes are already available for life scientists and its success depends on the involvement of physiologists and engineers from the design to the final application. This is a multidisciplinary field where collaboration will certainly pave the way for advancing our understanding of complex biological systems.

Micronanotechnologies, microfluidics, graphene, organ-on-achip, Neural interfaces,

\section{L2}

OPTOELECTRICAL DYNAMICS OF CALCIUM- AND VOLTAGE-GATED ION CHANNELS IN SUBCELLULAR CALCIUM NANODOMAINS

\section{Teresa Giraldez}

Universidad de La Laguna, La Laguna, Spain

In neurons, intracellular $\mathrm{Ca}^{2+}$ constitutes a major trigger of diverse yet crucial signaling events and reaction cascades. In order to carefully manage the complex set of $\mathrm{Ca}^{2+}$-dependent signals, intracellular $\mathrm{Ca}^{2+}$ concentration $\left(\left[\mathrm{Ca}^{2+}\right]_{\mathrm{i}}\right)$ is increased only for short periods of time in spatially restricted domains, where proteins mediating $\mathrm{Ca}^{2+}$ entry to the cell (mainly voltage-dependent $\mathrm{Ca}^{2+}$ channels, VDCC) are localized in close proximity to $\mathrm{Ca}^{2+}$ targets. Further diffusion of $\mathrm{Ca}^{2+}$ ions is efficiently limited by a variety of $\mathrm{Ca}^{2+}$ buffer systems. In many neuron types, it has been proposed that VDCC form $\mathrm{Ca}^{2+}$ nanodomains with large conductance voltage- and calcium-dependent potassium channels (BK, SLO1 or KCa1.1) coupling $\mathrm{Ca}^{2+}$ signaling to membrane potential. The interaction between $\mathrm{Ca}^{2+}$ influx and $\mathrm{BK}$ activation is involved in essential neuronal processes such as repolarization and hyperpolarization following the action potential (AP), dendritic $\mathrm{Ca}^{2+}$ spikes, and neurotransmitter release. Inherited defects in BK channels function lead to seizure and epilepsy, indicating that this coupling mechanism is crucial to regulate neuron excitability in the healthy brain. Similar processes occur in other tissues where BK channels play vital roles, such as endocrine and smooth muscle cells.

How does this coupling occur? There is a big knowledge gap on the intriguing structural mechanisms underlying the regulation of $\mathrm{BK}$ by $\mathrm{Ca}^{2+}$ and voltage, as well as how these effectors are coupled to pore opening. Functionally, BK channels are membranespanning alpha subunits homotetramers (which can assemble with various accessory subunits) with a "modular" structure: a transmembrane region that contains the voltage sensor, and a large $\mathrm{C}$ - 
terminal region that binds divalent cations including $\mathrm{Ca}^{2+}, \mathrm{Mg}^{2+}$, $\mathrm{Cd}^{2+}$ and $\mathrm{Ba}^{2+}$ at three different binding sites with distinct properties. Each alpha subunit contributes a tandem of Regulator of Conductance for $\mathrm{K}^{+}$(RCK1 and RCK2) domains to the channel tetramer, forming a "gating ring" structure. Despite the effort of many research groups, no structure of the whole channel is still available; thus, the study of BK channel function has been addressed by studying either the pore function (using electrophysiology) or the isolated gating rings (using biochemistry and, more recently, X-ray crystallography). During the past years, our group has been working extensively to study the specialized behavior of this channel in the whole protein complex, at the membrane, in order to determine the complete range of structures and movements critical to its in vivo function. I will present the results of our work in which, by using a combination of genetic approaches, fluorescence microscopy and electrophysiology we have been able to measure for the first time molecular motions of the gating ring in intact channels, in response to different cations and voltage, thus providing unprecedented insights in the function of these channels.

Ion channels, cellular excitability, local calcium signalling

L3

\section{CHRONODISRUPTION AND MELATONERGIC DYSFUNC- TION IN AGING AND DISEASE}

\section{Rüdiger Hardeland}

Faculty of Biology and Psychology, University of Goettingen, Goettingen, Germany

The pineal gland and its hormone melatonin are part of the circadian system, which is composed of countless cellular oscillators present in central and peripheral organs, in a partially hierarchical relationship, with a variable degree of autonomy. In mammals, retinal light/dark information is transmitted via the hypothalamic pacemaker, the suprachiasmatic nucleus ( $\mathrm{SCN})$, through a neuronal pathway to the pineal. In this gland, melatonin is preferentially synthesized at night and released to the circulation and, via the pineal recess, directly to the third ventricle. Although melatonin is also produced in numerous extrapineal sites and although their overall amount of melatonin exceeds by orders of magnitude the levels in pineal and blood, pineal melatonin is privileged as a circadian signal of darkness, because other organs contribute poorly to circulating concentrations. Melatonin does not only transmit this information to peripheral organs and oscillators, but also to the SCN, where it exerts effects of phase resetting and, in humans, sleep induction. Nocturnal melatonin levels typically decrease by age, however, with high interindividual variability. This variability is partially due to the fact that several pathologies also cause reductions of melatonin, as reported for stress- and painrelated disorders, coronary heart disease/myocardial infarction, some neurological disorders, diabetes type 2, several types of cancer and, most strongly, Alzheimer's disease (AD). Contrary to cases in which a successful therapy may allow return to normal levels, neurodegeneration causes irreversible reductions. In $\mathrm{AD}$, decreases in melatonin levels may not primarily result from pineal degeneration, but from that of the SCN and/or connecting pathways. This is reflected by a progressive breakdown of the circadian order, evident as reductions of circadian amplitudes, temporal imprecision of maxima and, finally, decomposition of circadian patterns and phase relationships between different rhythms. This kind of deterioration is also observed in the melatonin rhythm. Age-related malfunction of circadian oscillators and melatonin secretion are intertwined and may mutually aggravate each other. Even earlier in life, disease-associated reductions of melatonin that weaken signaling to central and peripheral oscillators may have consequences to health. Decreased or inappropriately phased melatonin levels represent one aspect of chronodisruption. This may occur under conditions of light-atnight, which causes a photic shutoff of melatonin synthesis, or after repetitive phase shifts. In addition to this classic type of chronodisruption, several other possibilities exist, which are based on genetic or epigenetic deviations. Various gene polymorphisms concerning components of cellular circadian oscillators as well as those of melatonin formation or signaling have been shown to to be associated with diseases and disorders. On the oscillator side, gene variants can affect the sleep phase in terms of morningness or eveningness, may cause circadian rhythm sleep disorders, and favor mood disorders with an etiology of circadian dysfunction, such as forms of bipolar and seasonal affective disorders. Overwhelming evidence exists for the association of genetically based circadian dysfunction with types of cancer. This is explained by the fact that several core oscillator components are antitumor factors. As another consequence, transformed cells have to epigenetically silence these circadian antitumor genes to be able to exist as cancer cells. Malfunction of melatonergic signaling can be caused by mutations that change agonist affinity of melatonin receptors, reduce membrane expression of these receptors, or lead to imbalances between their major signal transduction pathways, adenylyl cyclase inhibition and ERK1/2 activation. However, lossof-function is not the only possible cause of melatonin-associated health problems. Recent evidence strongly indicates that a diabetes type 2 risk allele (' $G$ allele' carrying SNP rs10830963) of the $M T N R 1 B$ melatonin receptor gene is consistently overexpressed in beta cells, where it excessively reduces cAMP/cGMP levels and, thus, impairs insulin secretion.

Aging, chronodisruption, circadian, melatonin, receptor polymorphism

\section{L4}

\section{AN UNFOLDING CONNECTION BETWEEN YOGA AND MUSCLE CONTRACTION}

\author{
Julio M. Fernández
}

Department of Biological Sciences, Columbia University, New York, USA

We do not know why the mechanical stretching of muscle tissues, such as during yoga, is so beneficial for humans. Current theories of muscle contraction propose that the power stroke of a myosin motor is the sole source of mechanical energy driving the sliding filaments of a contracting muscle. Amazingly, these models exclude titin, the largest protein in the human body, which determines the passive elasticity of muscles and is mechanically unfolded by stretching. During my lecture I will provide evidence at 
the single molecule level, that stepwise unfolding and folding of titin Ig domains occurs in the elastic I band region of intact myofibrils at physiological sarcomere lengths and forces of 6-8 $\mathrm{pN}$. Further, I will show that titin folding does mechanical work at these forces that is up to three times larger than that generated by a myosin motor. Therefore, it appears inescapable that folding of titin Ig domains is an important, but so far unrecognized contributor to the force generated by a contracting muscle. Thus, ancient wisdom was right again: recruiting titin domains to the unfolded state by stretching greatly increases the storage of elastic energy in muscle, which is an essential component of muscle contraction.

\section{SYMPOSIA}

S1-1

\section{DESIGN AND SYNTHESIS OF GOLD NANOPARTICLES} FOR THERAPY AND DIAGNOSIS

Jesús M De La Fuente

Instituto de Ciencia de Materiales de Aragon- CSIC/University of Zaragoza, Zaragoza, Spain

In the last decades, inorganic nanoparticles have been steadily gaining more attention from scientists from a wide variety of fields such as material science, engineering, physics or chemistry. The very different properties compared to that of the respective bulk, and thus intriguing characteristics of materials in the nanometre scale, have driven nanoscience to be the centre of many basic and applied research topics. Moreover, a wide variety of recently developed methodologies for their surface functionalization provide these materials with very specific properties such as drug delivery and circulating cancer biomarkers detection. In this talk we describe the synthesis and functionalization of gold nanoparticles as therapeutic and diagnosis tools against cancer:

- Pseudo-spherical gold nanoparticles derivatized with with fluorescent dyes, cell penetrating peptides and small interfering RNA (siRNA) complementary to the proto-oncogene myc have been tested using a hierarchical approach including three biological systems of increasing complexity: in vitro cultured human cells, in vivo invertebrate (freshwater polyp, Hydra) and in vivo vertebrate (mouse) model. Selection of the most active functionalities was assisted step by step through functional testing adopting this hierarchical strategy. ${ }^{1}$ Merging these chemical and biological approaches lead to a siRNA/RGD gold nanoparticle capable of targeting tumor cells in lung cancer xenograft mouse model, resulting in successful and significant c-myc oncogene downregulation followed by tumor growth inhibition and prolonged survival of the animals. ${ }^{2}$

- Gold nanoprisms (NPRs) have been functionalized with PEG, glucose, cell penetrating and RGD peptides, antibodies and/or fluorescent dyes, aiming to enhance NPRs stability, cellular uptake and imaging capabilities, respectively. ${ }^{3} \mathrm{Cel}-$ lular uptake and impact was assayed by a multiparametric investigation on the impact of surface modified NPRs on mice and human primary and transform cell lines. Under
NIR illumination, these nanoprobes can cause apoptosis. Moreover, these nanoparticles have also been used for optoacoustic imaging and cancer treatment, ${ }^{4}$ as well as for tumoral marker detection using a novel type of thermal ELISA nanobiosensor using a thermosensitive support. ${ }^{5}$

\section{References}

[1] J. Conde, A. Ambrosone, V. Sanz, Y. Hernandez, F. Tian, P. V. Baptista, M. R. Ibarra, C. Tortiglione, J. M. de la Fuente. ACS Nano, 2012, 6, 8316.

[2] J. Conde, F. Tian, Y. Hernández, C. Bao, D. Cui, M. R. Ibarra, P. V. Baptista, J. M. de la Fuente. Biomaterials. 2013, 34, 7744.

[3] a) B. Pelaz, V. Grazú, A. Ibarra, C. Magén, P. del Pino, J. M. de la Fuente. Langmuir, 2012, 28, 8965; b) M. Perez-Hernandez, P. del Pino, S.G. Mitchell, M. Moros, G. Stepien, B. Pelaz, W.J. Parak, E.M. Galvez, J. Pardo, J.M. de la Fuente. ACS Nano, 2015, 9,52

[4] a) C. Bao, N. Beziere, P. del Pino, B. Pelaz, G. Estrada, F. Tian, V. Ntziachristos, J. M. de la Fuente, D. Cui. Small, 2013, 9, 68; b) J. Han, J. Zhang, M. Yang, D. Cui, J.M. de la Fuente. Nanoscale, 2016, 8, 1704.

[5] E. Polo, P. del Pino, B. Pelaz, V. Grazu, J.M. de la Fuente. Chemical Communications, 2013, 49, 3676.

Nanoparticles, Therapy, Diagnosis, Nanomedicine

\section{S1-2}

BIOMIMETIC LAB-ON-A-CHIP MICRODEVICES TO RESEMBLE CELLULAR MICROENVIRONMENT IN PHYSIOLOGICAL CONDITIONS

Luis J Fernandez Ledesma, Jose María Ayuso, María Virumbrales, Guillermo A Llamazares, Alan Viguera, Alodia Lacueva, Marta Olave, Diego De Miguel, Rosa Monge, Manuel Doblare, Luis Martinez-Lostao, Ignacio Ochoa

Universidad de Zaragoza, Zaragoza, Spain

Cells are closely related with other cell types and with chemical and physical gradients generated in their microenvironments inside a normal tissue. It is well known that morphology, structure and function have always been related. Physiological response of cells is affected by their surrounding conditions. The cellular microenvironment has a major impact on the cells behavior and, for example, their response to chemotherapeutic agents. In tissues, the physiological microenvironment is characterized by complex heterotypic cell interactions and changing physiological gradients of nutrients, waste products and oxygen. Despite this, most in vitro cell research still relies primarily on cells grown in $2 \mathrm{D}$, isolated and with non-physiologically - rich conditions of nutrient- and oxygen.

Recently, microfabrication and microfluidics have arisen as promising technologies for the development of high-performance cell 
culture systems to mimic complex scenarios. The versatility of this microfluidic platform allows for different aspects of the microenvironment to be monitored and dissected. In these microdevices it has been able to combine epithelial cells with stromal, endothelial or immune cells embedded in a three-dimensional extracellular matrix following the tissue physiological distribution. Moreover, the microdevices versatility allows self-induced generation of oxygen and nutrient gradients as well as the appearance of a necrotic core, a hypoxic middle layer and a normoxic outer layer. Using this approximation it has been assessed how different tumors (e.g. brain, colon or breast tumors) behave differently in terms of proliferation or apoptosis in biomimetic conditions. Besides, it has been evaluated how different drugs (alkylating agents, TRAIL formulations or hypoxia-activated prodrugs) penetrate the tumor-associated endothelium to reach tumor cells and affect different layers. The device also potentially allows a live visual 'window' into the effects of the microenvironment on cancer cells. A major application of the device would be studying the effects of the microenvironment on drug responses and some data is presented for this indicating the amenability of the device and its potential to enable more physiological in vitro drug screening.

Biomimetic, microfluidic microdeviecs, cell microenvironment, physiological conditions

\section{S1-3}

\section{DEVELOPMENT OF AN IN VITRO EXPERIMENTAL MOD- EL TO STUDY THE MECHANICAL CHARACTERISTICS OF MUSCLE FATIGUE PHENOMENON. BIOENGINEERING APPLICATIONS}

\section{Marta Sierra Arregui, Jorge Grasa, Javier Miana-Mena}

Universidad de Zaragoza, Zaragoza, Spain

Skeletal muscle fatigue may be originated by repeated or sustained contractions of the tissue that diminish its maximum load levels. This phenomenon, known as fatigue, involves multitude of processes and physiological mechanisms that remain being an object of study and analysis. The in vitro mechanical studies allow monitoring muscle behaviour in a controlled environment. These muscle fatigue models provide useful information about development and extent of fatigue from a mechanical point of view. Thus, they are essential to create mathematical models that, in the future, could substitute the animals models in muscle fatigue studies. Computational models have been developed to predict the evolution of the force and to simulate the onset of fatigue. However, many of them only can fit the biological data obtained from the biological experiments. The aim of this work is to obtain the biological data necessary to develop a response surface that incorporates an important advantage in comparison with previous approximations because it allows to obtain new fatigue data from parameters not tested in animals. In order to obtain these biological data, authors have developed an in vitro muscle fatigue model of rabbit Extensor Digitorum Longus (EDL). This study was carry out on 12 male New Zealand White rabbits divided in four groups $(n=4)$ to obtain the EDL force evolution under different stimulation parameters. In all of animals, EDL right and left were used. Functional in vitro test were conducted in a methacrylate organ bath designed by the authors and the evolution of the load was recorded by means of an electro-mechanic universal testing machine. In order to allow the electrical stimulation a pair of platinum plate electrodes running the length of the isolated muscle on either side were connected to a CIBERTEC CS-20 electrical signal generator. Regarding the stimulation protocol, amplitude and duration of electrical signal were fixed to $100 \mathrm{~V}$ and $0,2 \mathrm{~s}$ respectively for all the groups. In the first group, EDL muscles were stimulated with a continuous signal with $100 \mathrm{~V}$ amplitude. The interval between train of pulses was $6 \mathrm{~s}$. All the other groups received the same pulse duration $(1 \mathrm{~ms})$ and the same rest time $(10 \mathrm{~s})$. However, the frequency was $40 \mathrm{~Hz}$ for the second group, $60 \mathrm{~Hz}$ the third group and $100 \mathrm{~Hz}$ the fourth one. All the muscles were stimulated for one hour. The peak maximum force evolution presented no significant differences among groups. Nevertheless, the Group 1 experimented the greatest decline showing at $t=1000 \mathrm{~s}$ a reduction of around a $87.5 \%$. The high precision of the acquisition system allows to register not only the force but also the evolution of each contraction, showing how muscle performance change and decline inside each stimulation. All these biological data allowed the development of a response surface that took into account not only the decay of the maximum repeated peak force, but also the shape evolution of each contraction, muscle weight, electrical input signal and stimulation protocol. This new approach of the fatigue simulation challenge permits to predict, inside the multi-space surface generated, the muscle response considering other stimulation patterns, different tissue weight, etc. Finally, the in vitro model developed here presented important avantages like total elimination of central fatigue or complete electrical activation of all the muscle fibers. Thus, it could be used to deeplier studies of muscle fatigue, including ionic factors, protocol variation, drug analysis, etc.

Skeletal muscle fatigue, in vitro models, mathematical models.

\section{S1-4}

BIOMECHANICAL PROPERTIES OF THE CORNEA IN A RABBIT MODEL OF AMOEBIC KERATITIS: USE OF CORNEAL COLLAGEN CROSSLINKING

Ángel Ortillés, Marta Sierra, Miguel Ángel Ariza-Gracia, María Benito, Jorge Belloc, Pilar Goñi, Begoña Calvo

University of Zaragoza, Zaragoza, Spain

Purpose: Acanthamoeba keratitis (AK) is a severe, painful and potentially sight-threatening infection of the corneal tissue. This disease is caused by a free-living protozoan, ubiquitous in a variety of habitats, and the main risk factor is contact lenses wear. Corneal collagen crosslinking (CXL) is a relatively new treatment option. In vitro studies have shown no amebicidal effect of riboflavin combined with UVA exposure, but some clinical case reports suggest a more promising picture with significant improvement in combination with topical medical therapies. Most of the mechanical tests described to determine the corneal biomechanical properties are performed in in vitro animal models. Corvis ST is the only device that provides the in vivo mechanical corneal response, but it has been described that the corneal deformation 
response to an air puff is dependent on intraocular pressure (IOP) and corneal thickness (CT) and not only of the corneal biomechanical properties. The aim of this study is to assess the corneal mechanical behavior in an AK experimental rabbit model after the infection and its treatment with CXL using a new in vivo indentation test and considering the IOP and CT effect.

Methods: AK was experimentally induced in the left eyes of eight male New Zealand white rabbits using silicone-hydrogel bandage contact lenses infected with trophozoites and cysts, after complete corneal epithelium debridement (right eyes were considered as control). Three strains of Acanthamoeba spp belonging to genotype T4 were used: P31 $(n=4)$ as an environmental amoeba isolated from superficial water, $G$ $(n=4)$ and $L(n=2)$ as clinical strains isolated via corneal scraping from patients with keratitis. After 24 days, a topical treatment with moxifloxacin $0.5 \%$, voriconazole $1 \%$ and clorhexidine $0.02 \%$ tree times a day was applied for 30 days. Furthermore, CXL $\left(3 \mathrm{~mW} / \mathrm{cm}^{2} ; 370 \mathrm{~nm}\right)$ for $30 \mathrm{~min}(5.6 \mathrm{~J} /$ $\left.\mathrm{cm}^{2}\right)$ was performed in three rabbits $(P 31, n=1 ; G, n=1 ; L$, $n=1)$ and topical non-steroidal anti-inflammatory (diclofenac $0.1 \%)$ was added in other three rabbits $(P 31, n=1 ; G, n=1$; $L, n=1)$ on the 20th day, being a rabbit $(L)$ treated with both. In vivo indentation tests were performed twice in each eye (corneal centre) before (Pre) and after beginning the treatment on the 20th (Post-20 day) and 30th day (Post-30d). IOP/CT were measured before each test.

Results and conclusions: After performing in vivo indentation tests previously and on the 20th and 30th day of the treatment, there were no statistically significant differences between infected (treated or untreated) and control eyes. Each rabbit was separately assessed because different treatments were used, and no variations of the mechanical properties of the corneal tissue were observed using CXL or topical non-steroidal anti-inflammatory. A slightly more compliant behaviour was detected in infected (treated or untreated) eyes but significant differences were not found. In conclusion, mechanical properties of the cornea assessed using in vivo indentation tests were not different after $\mathrm{AK}$ infection and its treatment with CXL.

Amoebic keratitis, rabbit model, corneal coliagen crosslinking, mechanical properties

\section{S2-1}

\section{A TSFM MUTATION CAUSES CHILDHOOD-ONSET ATAX- IA AND NON-OBTRUCTIVE CARDIOMYOPATHY}

Sonia Emperador Ortiz ${ }^{(1)}$, M. Pilar Bayona-Bafaluy ${ }^{(2)}$, Ana Fernández-Marmiesse ${ }^{(3)}$, Mercedes Pineda ${ }^{(4)}$, Blanca Felgueroso (5), Ester López-Gallardo (1), Rafael Artuch (4), Iria Roca ${ }^{(3)}$, Eduardo Ruiz-Pesini ${ }^{(6)}$, María Luz Couce ${ }^{(3)}$, Julio Montoya ${ }^{(1)}{ }^{\text {' }}$

(1) CIBERER-Universidad de Zaragoza, Zaragoza, Spain; (2) Instituto de Investigación Sanitaria de Aragón (IIS Aragón). Universidad de Zaragoza, Zaragoza, Spain; ${ }^{(3)}$ Hospital Clínico Universitario de Santiago de Compostela. Instituto de Investigación Sanitaria de Santiago de Compostela (IDIS), Santiago de Compostela, Spain; ${ }^{(4)}$ Hospital San Joan de Deu. Institut de Recerca Pediàtrica (IRP-HSJD), Barcelona, Spain; ${ }^{(5)}$ Hospital Materno Infantil Teresa Herrera., A Coruña, Spain; ${ }^{(6)}$ Fundación ARAID. Universidad de Zaragoza, Zaragoza, Spain

Background: The oxidative phosphorylation system (OXPHOS) is a biochemical pathway involved in many key cellular processes. This system includes electron transport chain (ETC.) respiratory complexes I - IV (CI-CIV) and ATP synthase (CV). OXPHOS complexes include 13 mitochondrial DNA (mtDNA)-encoded polypeptides. Moreover, mtDNA also codes for 22 transfer and 2 ribosomal RNAs required for these polypeptides expression. Mitochondrial translation also depends on many nuclear DNA (nDNA)-encoded proteins. Therefore, mutations in components of mitochondrial translation system can be responsible for different pathologies involving an OXPHOS dysfunction.

Methods: We describe a patient suffering from slowly progressive childhood-ataxia and hypertrophic cardiomyopathy. To unravel the etiologic factor of this phenotype, we used different cell, biochemical and molecular-genetic protocols.

Results: A homozygous missense mutation was identified in the mitochondrial translation elongation factor Ts (TSFM) gene of this patient. We confirmed its pathogenicity by cellular and molecular techniques, including the rescue of a normal phenotype of his fibroblasts by transfecting them with the wild-type allele.

Conclusions: The deficiency in activity of several respiratory complexes with mtDNA-encoded subunits, the altered mitochondrial protein synthesis, the EFTs absence in patient fibroblasts and the correction of the biochemical defect by overexpressing the wild-type EFTs confirm that the reported EFTs mutation is the etiologic factor the for phenotype of this patient.

Several mutations in nuclear-encoded translation elongation factors and in other proteins involved in mitochondrial translation have been recently reported thanks to the Next-generation sequencing methodology. These techniques seem to be an efficient way to find new diseasecausing mutations in mitochondrial patients with variable clinical manifestations.

Mitochondrial disease, OXPHOS, EFTs, Mutation.

\section{S2-2}

HEREDITARY SPASTIC PARAPLEGIA (HSP), AN ENDOPLASMIC RETICULUM DISEASE

Marta Palomo, Rut Fadó, Beatriz Moreno, Rosalía RodríguezRodríguez, Núria Casals

Universitat Internacional de Catalunya, Sant Cugat del Vallés, Spain

Hereditary spastic paraplegias (HSPs) are a group of inherited neurological disorders characterized by slowly progressive weakness and spasticity of the muscles of the legs. Affected individuals will experience progressive difficulty in walking and may eventually require an assistive device such as a cane or a walker, or in severe cases, a wheelchair. The symptoms are caused by a lengthdependent axonopathy that affects mainly corticospinal and lower motor neurons, whose axons are among the longest in the body. To date, more than 50 spastic paraplegia genes (SPGs) have been identified. Despite this genetic heterogeneity, the functions of the 
encoded proteins converge on a small number of common aspects, all related with endoplasmic reticulum (ER), such as lipid metabolism, ER morphology, mitochondrial dynamics, and endosomal trafficking.

We have recently published the case of an Italian family with 5 affected individuals that showed a mutation in carnitine palmitoyltransferase $1 \mathrm{C}(\mathrm{CPT} 1 \mathrm{C})$ gene, causing a pure form, adult onset of dominant HSP, termed SPG73 (Rinaldi et al., JAMA neurology, 2015). This is the first disease-causing Cptlc mutation described in humans to date. Whole exome sequencing of genomic DNA allowed the identification of a single nucleotide substitution c.109C $>$ T (p.Arg37Cys) in Cpt1c of affected individuals. Interestingly, CPT1C deficient mice showed motor deficit symptoms similar to the human ones: severe muscle weakness, impaired coordination and gait, and reduced daily locomotor activity. Moreover, motor deficits appeared at 6-7 weeks of age (young adult mice) and progressively increased with time.

CPT1C is a pseudoenzyme that is expressed exclusively in neurons and localizes in ER of the cells, contrary to the mitochondrial isoforms (CPT1A and CPT1B). Despite not having catalytic activity, CPT1C maintains the ability to bind malonyl-CoA, a fatty acid synthesis intermediary, whose intracellular concentration drops dramatically in energetically deficient situations. This fact suggests that CPT1C can be a malonyl-CoA sensor in neurons that regulates the function of other interacting proteins depending on the energetic status of the neuron.

We have demonstrated the interaction of CPT1C with two other ER transmembrane proteins, Atlastin-1 and Protrudin, which are two of the most frequently mutated proteins causing HSP (SPG3A and SPG33, respectively). Mutation or deficiency of any of these two proteins or CPT1C results in impaired axon growth, suggesting that the three proteins work coordinately in axon elongation.

Atlastin-1 is a GTPase required for homotypic fusion of ER membranes, which is involved not only in ER morphology but also in the regulation of lipid droplet (LD) size. The main function of LDs is to serve as reservoirs of cholesterol and acyl-glycerols for cell membranes formation and maintenance. Their microtubule-mediated movement assures lipid availability in specific regions during membrane expansion. We have demonstrated that CPT1C also promotes triacylglycerol synthesis and LD biogenesis in cultured neurons. Therefore, we postulate that CPT1C binds to and regulates the function of Atlastin-1 in LD dynamics in response to changes in intracellular malonyl-CoA levels.

On the other hand, it has recently been described that Protrudin plays a key role in the regulation of LE transport along the axon through the LE-ER contact sites. Protrudin delivers Kinesin-1 to LE, thereby equipping the LE with the ability to move along microtubules in the plus-end direction (towards the cell periphery), and promoting their fusion with the plasma membrane, resulting in neurite growth. Interestingly, not published results from our group demonstrate the involvement of CPT1C in LE positioning in the cell. Our hypothesis is that CPT1C regulates Protrudin function and controls LE trafficking depending on the metabolic status of the neuron.

The identification of $C p t 1 c$ as a new SPG confirms the relevance of ER in functionality of longer axons.
Axonopathy, carnitine palmitoyltransferase 1C (CPT1C), Atlastin1, Protrudin, corticospinal neurons, malonyl-CoA

THE COHESIN COMPLEX FAILURE MAY CAUSE CORNELIA DE LANGE SYNDROME

Juan Pié Juste

Unit of Clinical Genetics and Functional Genomics, Department of Pharmacology and Physiology, School of Medicine, University of Zaragoza, CIBERER-GCV and IIS-Aragon, Zaragoza, Spain

The cohesin complex is a protein machine that is responsible for maintenance of DNA. Although its origin is found in prokaryotes, is in eukaryotes when it diversifies and produces the complexes of condensin, cohesin and SMC5-SMC6. From a structural point of view is ring-shaped and consists of four proteins: SMC1A / SMC1B, SMC3, RAD21 / REC8 / RAD21L and STAG1 / STAG2 / STAG3 and a large number of regulatory proteins as NIPBL, ESCO2 or HDAC8. The first known function of the complex was the cohesion of sister chromatids during the cell cycle. But at present, it is known that is critical in DNA replication and repair, the compartmentalization of this molecule in the nucleus, and especially in the regulation of gene expression. Alterations of this complex produce a new group of pathologies called cohesionopaties including, among others, Warsaw breakage syndrome, Roberts / SC phocomelia syndrome or Cornelia de Lange Syndrome (CdLS). Although these syndromes have a varied clinic, they all have in common cause alterations of form and intellectual disability. The best known is Cornelia de Lange syndrome that is caused by mutations in genes encoding NIPBL, SMC1A, HDAC8, SMC3 and RAD21 proteins. However, the most common cause are mutations of the regulatory protein NIPBL. Recently, it has been reported that adherina, protein complex formed by NIPBL more MAU2, is responsible for maintaining free of nucleosomes the DNA strand. This action is essential for the cohesin ring can embrace the DNA molecule, and approximates the NIPBL function to other protein complexes chromatin remodelers as RSC (Chromatin Structure Remodeling). Although the clinical syndrome is well known and includes: a characteristic facies, delayed growth pre and postnatal, intellectual disability and impaired limb; the production mechanism of the syndrome is still unclear. Currently, it is believed that the regulation of gene expression fails, especially homeotic genes. Thus in drosophila it has been shown that cohesin produces Polycomb gene silencing, fundamental in the control of genes that regulate the shape. It has also been described that could be a downregulation of $c-M y c$ protooncogene. This gene is one of the keys of cell proliferation and could be one of the first to be affected in these patients.

\section{S2-4}

HUMAN HMG-COA LYASES: TWO GENES, THREE ENZYMES AND ONE PATHOLOGY

Beatriz Puisac Uriol (1), Esperanza Teresa Rodrigo ${ }^{(1)}$, María Hernández Marcos ${ }^{(1)}$, M ${ }^{\mathrm{a}}$ Concepción Gil-Rodríguez ${ }^{(2)}$, Javier Santiago $\operatorname{Arcos}^{(1)}$, Juan Pié Juste ${ }^{(1)}$ 
(1) Universidad de Zaragoza, Zaragoza, Spain; ${ }^{(2)}$ Certest Biotec, Zaragoza, Spain

There are two different genes which encode three different human HMG-CoA lyase enzymes. The first one, discovered in 1993, was the $H M G C L$ gene located on chromosome 1. It contains nine exons and eight introns. The HMGCLL1 gene has been identified in 2012 on chromosome 6 and has eleven exons and ten introns.

The HMGCL gene encodes two isoforms of the HL protein, one mitochondrial and the other peroxisomal, and both exhibit lyase activity. The mitochondrial isoform (mHL) has 298 aminoacids and has been deeply studied. Its structure is a homodimer TIM-barrel, joined by di-sulfur bridges. In this organelle, it catalyzes the cleavage of 3-hydroxy- 3methylglutaryl CoA (HMG-CoA) to acetoacetate and acetyl$\mathrm{CoA}$, a key reaction in ketogenesis and leucine catabolism. Traditionally, ketone bodies have been considered to act as an alternative energy source to glucose, especially for the brain. But recent findings have also linked them to diverse functions as lipogenesis, neuroprotection and longevity regulation through inhibition of histone deacetylases. It is mainly expressed in liver, the ketogenic tissue par excellence, but it has been reported to be expressed and active in other human tissues such as pancreas, kidney or skeletal muscle. It has been proposed that this enzyme and its predecessor in ketogenesis pathway, HMG-CoA synthase, would be regulated by alternative splicing coordinately.

The function of the peroxisomal isoform is still unknown. It has been proposed to play a role in cholesterol synthesis or in long-chain fatty acid degradation. It gets into the peroxisomes due to the CKL signal tripeptide of the C-terminal end. The peroxisomal isoform is larger than the mitochondrial one ( 325 aminoacids) because of the presence in its structure of the mitochondrial signal peptide. Despite the differences in size and amino acid sequence between the two isoforms, their kinetic activity values of $\mathrm{Km}$ and kcat are close and both have homodimeric structure.

The HMGLL1 gene encodes the third HMG-CoA lyase enzyme, the er-cHL. Our group reported in 2012 that this enzyme shows a dual location in cytosol and endoplasmic reticulum. It presents structural homology with the mitochondrial enzyme but its $\mathrm{N}$-terminal sequence suggests a myristoylation site. However, its kinetic characteristics are different, with a highest affinity and lower Vmax than mHL. It also shows differences in tissue expression, with detectable levels in lung, kidney, heart and adult and fetal brain. This specific tissue localization could adressed a function related to lipid synthesis in lung or brain, but its real function still must be demonstrated.

So far, only an inborn error of metabolism by mitochondrial HL deficiency has been described, that appears with hypoglycemic states and is accompanied by hypoketosis and metabolic acidosis. This rare disease is inherited in an autosomal recessive manner and has been described in about 100 patients. The consequences of the deficiency of the other two HL have not been described and new functional or animal model experiments should be performed.

Ketone bodies, lyase activity, endoplasmic reticulum-cytosolic HMG-CoA lyase, mitochondrial HMG-CoA lyase, peroxisomal HMG-CoA
S3-1

\section{THE REELIN IN THE INTESTINE}

Ana Ilundain

Facultad de Farmacia, Sevilla, Spain

Reelin is a secreted extracellular matrix protein critical for neuronal migration in the developing brain. We found that within the mucosa of rodent intestine and human colon, reelin expression is restricted to the myofibroblasts whereas its receptors (apolipoprotein $\mathrm{E}$ receptor 2 (ApoER2) and very low density lipoprotein receptor) and effector protein disabled-1 are in myofibroblasts and epithelial cells [1-3].

The absence of reelin (reeler mice) affects the epithelial renewal processes. It reduces cell proliferation, migration, apoptosis and the number of Paneth cells in the small intestine and that of Goblet cells in the colon. It also expands the extracellular space of the adherens junctions and desmosomes, which might indicate loosening of the junctions [4]. In addition to hamper the defence barrier of the intestine against external agents, the largest number of differentially expressed genes modified by the reeler mutation is related with intestinal immune response, inflammation and tumour development [5].

All these observations prompted us to suggest that reelin may protect the intestine from development of pathologies. To corroborate this hypothesis the expression of the reelin signalling system was measured in the colon of mice treated either with DSS (colitis) or with DSS plus AOM (cancer) and in human colorectal cancer. Reeler mice has higher susceptibility to develop colitis and colon cancer than wild type mice but the response of the reelin differs within the two pathologies. DSS-treatment increases reelin expression within the mucosa to a level much greater than that of the myofibroblasts marker $\alpha$-SMA (smooth muscle actin), indicating that reelin up-regulation results from activation of reelin gene transcription rather than from increased number of myofibroblasts. Activation of reelin transcription in colon differs from that in brain, wherein it is up-regulated by the transcription factors $\mathrm{Sp} 1$ and Tbr1 (T-brain-1)/Cask (calcium/calmodulin-dependent serine protein kinase), because Tbr1 is not expressed by mice colon and colitis down-regulates Sp1 and Cask mRNAs. Up-regulation of reelin is accompanied by increased TGF- $\beta 1$ (transforming growth factor $\beta 1)$ mRNA, a decrease in that of DNMT1 (DNA (Cytosine-5-)Methyltransferase 1) and by hypo-methylation of the reelin promoter region. These findings suggest that activation of reelin expression results from DNMT1-dependent hypo-methylation of its gene promoter region, which in turn may be provoked by the increase in TGF- $\beta 1[3,6,7]$.

Human and mice colon cancer down-regulate reelin, that is accompanied by a slight decrease in $\alpha$-SMA mRNA levels. The reelin down-regulation observed in human occurs without change in Cask or TGF- $\beta 1 \mathrm{mRNA}$ levels, but it is accompanied by increased levels of DNMT1 and ApoER2 mRNAs and decreased levels of HIC1 (Hypermethylated In Cancer 1) mRNA. Based in other reports $[8,9]$, our observations suggest that decreased HIC1 expression activates that of ApoER2, which in turn would repress reelin expression. Therefore, the cancer associated reelin downregulation might result from DNMT1-mediated hypermethylation of its gene promoter and from HIC1-dependent increase in ApoER2 abundance [2 and unpublished observations]. 
Grant from the Junta de Andalucía (CTS 5884)

Key words: reelin, ApoER2, colitis, cancer

1. García-Miranda et al. Exp Physiol, 2010, 95: 498-507

2. García-Miranda et al. Genes Nutr, 2012,1: 51-81

3. Peral et al. J Physiol Biochem, 2013, 69: 670-671.

4. García-Miranda et al. Tissue Eng, 2013, 19: 188-98.

5. García-Miranda et al. J Physiol Biochem, 2012, 68: 205-18

6. Ilundain et al. Acta Physiol, 89, 2014

7. Peral et al. Acta Physiol, 97, 2015

8. Dubuissez et al. Biochem Biophys Res Commun 2013, 440: 424-430.

9. Balmaceda et al. FASEB J, 2014, 28:1543-155

Reelin, intestine, colitis, cancer

S3-2

OMEGA-3 FATTY ACIDS AND THEIR DERIVED LIPID MEDIATORS AS BLOCKERS OF TNF-A-INDUCED DECREASE OF NUTRIENTS TRANSPORT

Rosa Castilla-Madrigal, Marta López De Calle, María Jesús Moreno-Aliaga, María Pilar Lostao

University of Navarra, Pamplona, Spain

Inflammatory Bowel Disease (IBD) is characterized by chronic inflammation of the gastrointestinal mucosa, presenting high levels of tumor necrosis factor-alpha (TNF- $\alpha$ ). During intestinal inflammation, the expression and activity of many nutrients and electrolytes transporters can be modified, which may explain the characteristic malabsorption and diarrhea found in IBD patients. Dysfunctional inflamed mesenteric adipose tissue in obesity could also contribute to the chronic intestinal inflammation in IBD, leading to a more active and severe development of the disease. We have previously demonstrated in Caco-2 cells that TNF- $\alpha$ inhibits sugar uptake by decreasing the amount of the $\mathrm{Na}^{+}$-glucose cotransporter SGLT1 in the brush border membrane. The omega-3 fatty acids (n-3 PUFAs), eicosapentaenoic acid (EPA) and docosahexaenoic acid (DHA) and their lipid mediators, resolvins (Rv), protectins and maresins (MaR), are able to correct intestinal inflammation in models of IBD. However, their ability to counteract the alterations of intestinal absorption of nutrients induced by inflammation remains unknown. GPR120 is a G proteincoupled receptor, widely expressed, that acts as a sensor/receptor of EPA and DHA.

The aim of the present study was to investigate in Caco-2 cells whether EPA, DHA and its derived lipid mediators RvD1, RvD2 and MaR1 are able to block the inhibitory effect of TNF- $\alpha$ on sugar and glutamine transport. The intracellular pathways involved were also studied.

Functional studies were performed using Caco-2 cells grown as a monolayer. Apical uptake of alpha-methyl-glucoside $(\alpha \mathrm{MG})$ or glutamine was measured, after preincubating the cells with TNF- $\alpha$ in the absence or presence of the different n-3 PUFAs or lipid mediators. The expression of the nutrients transporters in the apical membrane of the Caco-2 cells was analyzed by Western blot.
TNF- $\alpha$ inhibits $\alpha M G$ uptake by decreasing SGLT1 expression in the plasma membrane through the activation of ERK1/2 and inhibition of AMPK pathways. EPA prevents the inhibitory effect of TNF- $\alpha$ on $\alpha$ MG uptake through GPR120 and AMPK activation. This effect is accompanied by the lack of modification of SGLT1 expression in the membrane. DHA and RvD1, RvD2 and MaR1 also blocked the decrease of sugar transport induced by TNF- $\alpha$, being DHA effect GPR120-independent. Likewise, EPA and DHA blocked TNF- $\alpha$ induced inhibition of glutamine transport. Similar results were obtained with MaR1 and RvD2.

Basolateral presence of TNF- $\alpha$ also reduces $\alpha \mathrm{MG}$ and glutamine uptake from the apical membrane. Interestingly, Caco-2 cells incubated in the apical side with conditioned medium obtained from Caco- 2 cells preincubated with basolateral TNF- $\alpha$, showed decrease on $\alpha \mathrm{MG}$ and glutamine uptake as well, suggesting TNF- $\alpha$ stimulation of proinflammatory cytokines secretion by the cells. In summary, n3-PUFAs and its derived-lipid mediators can block the decrease on sugar and glutamine uptake induced by TNF- $\alpha$. Therefore, these molecules could be beneficial in patients that suffer malabsorption associated with intestinal inflammation.

Omega-3, TNF- $\alpha$, nutrients transport

S3-3

PENDING QUESTIONS ON THE CONTROL OF INORGANIC
PHOSPHATE HOMEOSTASIS

Víctor Sorribas

Universidad de Zaragoza, Zaragoza, Spain

In spite of the important knowledge obtained for the last 20 years, the complete scenario of phosphate homeostasis control is far from being understood, in spite of the physiological and pathophysiological importance.

Under neutral balance, the intestinal absorption of phosphorus is similar to the phosphorus loss through the urine and other minor routes. A significant part of Pi absorption and most of renal reabsorption takes place transcellularly thanks to the involvement of sodium-coupled Pi transporters in the apical membranes. These transporters belong to two families: Slc34 which include type II transporters NaPi2a, b and c, and Slc20, which include the type III, PiT1 and PiT2. NaPi2a (Slc34a1) and NaPi2c (Slc34a3) are mainly expressed in the proximal tubules of the kidney, while $\mathrm{NaPi} 2 \mathrm{~b}$ (Slc34a2) is most abundant in the small intestine, liver and lung. PiT1 (Slc20a1) and PiT2 (Slc20a2) are ubiquitously expressed, even concomitantly with type II transporters, for unclear reasons. The mechanism of phosphate exit from the cell is also unknown, with the exception of the putative Pi equilibrator XPR1.

All Pi transporters but NaPi2c are rheogenic and $\mathrm{pH}$ dependent, with different characteristics based on the polyprotic dissociation of phosphate: The type II transports $\mathrm{HPO}_{4}{ }^{=}$and therefore the activity increases with alkalinity; type III transporters, however, prefer $\mathrm{H}_{2} \mathrm{PO}_{4}^{-}$, and the activity increases with acidity. The affinities for $\mathrm{Pi}$ are very similar, ranging $0.1-0.2 \mathrm{mM} \mathrm{Pi}$, with the exception of $\mathrm{NaPi} 2 \mathrm{~b}(10-15 \mu \mathrm{M} \mathrm{Pi})$.

In the kidney, most of Pi reabsorption can be explained by NaPi2a, because the characteristics of the total Pi transport are identical to the characteristics of $\mathrm{NaPi}$ a when expressed in Xenopus laevis 
oocytes. The need for NaPi2c, PiT1 and PiT2 expressions in the same cells is unknown. The situation in the intestine is more puzzling because the transport characteristics of the small intestine do not fit the characteristics of the individually expressed transporters.

Pi transporters do not work individually, instead they are connected to other proteins. Type II transporters mainly interact with PDZ domain-containing proteins, such as NHERF1-4, PIST, etc. The interaction with NHERF1 (EBP50) keeps the transporter in the plasma membrane, while the interaction with NHERF3 (PDZK1) is associated with internalization and degradation, and when interacts with PIST the expression in the trans-Golgi is increased. These proteins work as scaffolds, connecting the transporters to the cytoskeleton, and helping to the movement of the transporters towards and from the membrane with unclear mechanisms, because recycling of these transporters does not occur.

Phosphate homeostasis is therefore controlled by adjusting the rate of intestinal absorption and bone mobilization, and mainly, the rate of renal reabsorption and excretion. This control is handled through hormonal and non-hormonal mechanisms. The adaptation to the phosphate concentration of the diet is the main nonhormonal mechanism: Pi deprivation increases intestinal absorption and renal reabsorption of $\mathrm{Pi}$, while the reverse occurs with the high Pi diet. However, the molecular mechanisms of the regulation are still unclear, as well as the nature and location of phosphate sensor. Two hormones act as major phosphaturic agents: the phosphatonin FGF23 synthesized by the osteocytes, and the parathyroid hormone. FGF23 mainly acts in the kidney, but also peripherally with unclear effects, as it is the interrelation with PTH. Klotho is a membrane protein that interacts with the FGF23 receptor to increase sensitivity to FGF23, but a soluble Klotho isoform seems to have multiple effects in other tissues, including direct phosphaturic activity. Thyroid hormone, insulin, corticoids, vitamin $\mathrm{D}_{3}$, etc. are also agents with effects on $\mathrm{Pi}$ homeostasis.

In conclusion, the increasing number of questions and the relationship with several fatal disorders strongly suggest the need for more research in this field.

Inorganic phosphate, transporters, phosphatonin, vascular calcification, intestine, kidney

\section{S3-4}

\section{LPS-SQUALENE INTERACTION ON D-GALACTOSE IN- TESTINAL ABSORPTION}

$M^{\mathrm{a}}$ José Felices, Sara Escusol, Sonia Gascón, Roberto MartínezBeamonte, Cristina Barranquero, Jesús Osada, $\mathrm{M}^{\mathrm{a}}$ Jesús Rodríguez-Yoldi

\section{Fac. Veterinaria. Univ. Zaragoza, Zaragoza, Spain}

Bacteria belonging to the species Escherichia coli are often found in the intestinal flora of humans and animals. However, certain strains may cause significant digestive disorders related to intestinal motility and absorption. It is well known that the bacterial toxins can trigger sepsis syndrome. The septic state can be induced naturally or experimentally and can cause alterations in different physiological functions of the small intestine affecting its most important function which is the absorption of nutrients. Lipopolysaccharide (LPS) is a complex polymer forming part of the outer membrane of Gram negative bacteria.

Olive oil, the main source of the fat in the Mediterranean diet, is a functional food which besides having high amounts of monounsaturated fatty acid contains minor components with biological properties such as squalene. This component is a natural lipid belonging to the terpenoid family and a precursor of cholesterol biosynthesis. Because of its significant dietary benefits, squalene contributes as a protective and preventive agent in cancer treatment (colon, breast, skin), bacterial and fungal infection, coronary heart disease and ageing by inhibiting oxidative stress.

The aim of this work was to study if rabbit feed with a rich squalene diet and/or Caco-2 epithelial intestinal cells treated with this triterpene could change the LPS effect on intestinal absorption of D-galactose.

Rabbit was chosen as experimental model because these animals have a similar monocavitary digestive system to humans. Therefore, the results can be extrapolated. With this model, we used two different ways to infection: acute (intravenous administration of LPS) and chronic (the endotoxin was administrated through intraperitoneal osmotic pump). Animals were divided in two groups: standard or rich squalene diet.

At the same time, we used Caco-2 epithelial intestinal cells to study LPS and squalene effects in the D-galactose intestinal transport without the influence of neural and hormonal signals, which are present in the animal model. Caco-2 cells are a human intestinal cell line that was isolated from a colon carcinoma. These cells differentiate into polarized monolayer such that their phenotype, morphologically and functionally, resembles that of the small intestinal epithelial cells. They develop the morphological characteristics of mature enterocytes.

The results showed that squalene reduces the effects of bacterial sepsis induced by LPS in rabbits regarding processes fever and intestinal galactose malabsorption.

High LPS doses increased D-galactose uptake through paracellular via but also decreased the active sugar transport because the protein levels of SGLT1 were diminished. At the same time, we observed an increased in protein expression of Resistin-like molecule- $\beta$ (RELM- $\beta$ ) which could cause a decrease of the protein levels of SGLT1. Moreover, we used bioinformatics programs which showed a possible interaction between SGLT1 and RELM- $\beta$. We also studied the interaction between LPS and MLCK, protein of paracellular way. The results showed that the endotoxin could activate MLCK and produce an increase of sugar transport through tight junctions. Bioinformatics studies showed a possible interaction between LPS and SGLT1. This interaction and the activity of several kinases could also modify the activity of SGLT1, inducing a reduction in the relative intrinsic activity of SGLT1. It could explain the decrease sugar absorption found at low LPS doses. The effect of LPS on paracellular way seems to be more important than on the transcellular route.

Studies on Caco-2 cells confirmed the results obtained in rabbits. In conclusion, squalene could inhibit the effect of LPS on Dgalactose intestinal absorption through NF-kB factor and MLCK protein.

LPS, squalene, intestinal-absorption, SGLT1, RELM-beta, MLCK 
S3-5

ROLE OF THE SEROTONIN TRANSPORTER IN THE PATHOPHYSIOLOGY INTESTINAL

\author{
Elena Layunta ${ }^{(1)}$, Eva Latorre ${ }^{(2)}$, José Emilio Mesonero ${ }^{(1)}$
}

${ }^{(1)}$ Departamento Farmacología y Fisiología, Facultad de Veterinaria, Instituto de Investigación Sanitaria de Aragón (IIS), Universidad de Zaragoza, Zaragoza. Spain. Instituto Agroalimentario de Aragón IA2- (Universidad de Zaragoza - CITA), Zaragoza, Spain; ${ }^{(2)}$ RNA Mediated Mechanisms of Disease. University of Exeter Medical School, Exeter, United Kingdom

The intestinal epithelium forms a physical barrier, but also acts as an immunological barrier against toxins and microorganisms from the intestinal lumen that helps to maintain homeostasis of the body, and particularly on the intestine. Crosstalk between microbiota and host is mediated by recognition receptors expressed in both immunological and non-immunological host cells. Toll-like (TLRs) and NOD-like (NLRs) receptors belong to this system; they are activated by binding of microorganism associated molecular patterns, and then they trigger different intracellular reactions. Intestinal microbiota-host interaction may be unbalanced either by a deregulation of recognition receptors or by dysbiosis. As consequence of this unbalance, chronic intestinal inflammatory diseases may be triggered.

In addition to the defensive barrier function, an important enteroendocrine activity arises from intestinal epithelium. Among the gastrointestinal hormones, serotonin (5-hidroxytrytamine, 5HT) is an essential intestinal neuromodulator that regulates the whole intestinal physiology including motility by a complex neuro-endocrine-immunological system. 5-HT is mainly synthesized in enterochromaffin (EC) cells in the intestinal epithelium by the tryptophan hydroxylase-1 (TPH1), whereas the isoform TPH2 is used by the serotonergic neurons of the enteric nervous system (ENS) to synthesize small amount of 5-HT. Extracellular 5-HT binds to different specific receptors, widely distributed throughout the gastrointestinal tract, to trigger their regulatory effects; however, 5-HT activity depends on the extracellular 5-HT availability that is mainly modulated by the serotonin transporter (SERT) expressed in the enterocytes, in both apical and basolateral membranes. SERT is responsible for the 5-HT uptake into these cells, thus finishing 5-HT effects. Intestinal serotoninergic system has been shown to have a key role in intestinal homeostasis, but also in the origin and/or consolidation of chronic gastrointestinal diseases such as inflammatory bowel diseases (IBDs). 5-HT level has been shown to be altered in experimental intestinal inflammation and in IBD patients, and high levels of 5-HT have also been described in several inflammatory and diarrheal conditions. Moreover, recent results have shown that pro-inflammatory molecules induced SERT inhibition, whereas IL-10, an anti-inflammatory cytokine increases SERT activity and expression. Furthermore, several TLRs are able to regulate intestinal SERT activity and expression by different mechanisms. Thus, TLR4 activation by LPS inhibits SERT activity by posttranscriptional mechanisms involving PKC, and TLR3, which is an intracellular TLR activated mainly by viral components, was shown to modulate SERT activity by activating the p38-MAPK pathway. Then, SERT inhibition may increase 5HT extracellular levels and contribute to the inflammatory response, acting as a pro-inflammatory molecule.
In summary, intestinal epithelial cells express different TLRs and NLRs in order to recognize microorganisms, and receptors for pro and anti-inflammatory factors, but also express SERT and 5-HT receptors to regulate 5-HT bioavailability and biological effects, respectively. Therefore, interrelationships between these different elements in the enterocytes seems to play an important role in the intestinal immune response, modulating the serotoninergic system, which in the end may contribute to the control of gut physiology or inflammatory pathologies.

Supported by MICINN-ERDF (BFU2009-08149; BFU201018971), DGA-ESF (B61), Zaragoza University (UZ2014-BIO03), and ARAINF (2012/0567)

Footnote: In Memoriam: This work is dedicated to the late Professor Anabel Alcalde.

SERT, 5-HT, INTESTINAL EPITHELIUM, INFLAMMATION, GUT

S4-1

ESSENTIAL ROLE OF $\mathrm{CA}^{2+}$ HOMEOSTASIS DYSREGULATION IN THE ADVERSE CARDIAC REMODELING DUE TO HEART REVASCULARIZATION

Alejandro Dominguez Rodriguez ${ }^{(1)}$, Ignacio Diaz ${ }^{(1)}$, Eva Sánchez De Rojas-De Pedro ${ }^{(1)}$, Jean Pierre Benitah ${ }^{(2)}$, Ana María Gómez ${ }^{(2)}$, Antonio Ordoñez ${ }^{(1)}$, Tarik Smani ${ }^{(1)}$

(1) Instituto de Biomedicina de Sevilla, Sevilla, Spain; ${ }^{(2)}$ Inserm S1180- Université Paris-Sud Châtenay, Paris, France

Acute myocardial infarction (AMI) due to coronary artery occlusion represents a major cause of morbidity and mortality in humans. Adverse ventricular remodeling occurs both at the site of AMI and in remote zone leading to heart failure (HF). HF is characterized by systolic and diastolic dysfunction, abnormalities in the intracellular $\mathrm{Ca}^{2+}$ concentration $([\mathrm{Ca} 2+] \mathrm{i})$ handling and the activation of $\mathrm{Ca}^{2+}$-dependent transcription factors related to adverse cardiac remodeling. However, little is known about $[\mathrm{Ca} 2+] \mathrm{i}$ changes in infarcted and remote zones induced by ischemia and reperfusion $(\mathrm{I} / \mathrm{R})$ injuries.

We analyzed the $\left[\mathrm{Ca}^{2+}\right] \mathrm{i}$ in a model of $\mathrm{I} / \mathrm{R}$ at 1 week in which we ligated the left anterior descending coronary artery (LAD) for $40 \mathrm{~min}$ and then circulation was reestablished. We comprised $\left[\mathrm{Ca}^{2+}\right] \mathrm{i}$ changes in cardiac myocytes isolated from remote and infarcted zone.

To induce the stenosis a 6/0 Prolene ${ }^{\mathrm{TM}}\left(\right.$ Ethicon $\left.^{\mathrm{TM}}\right)$ nylon suture was placed around the LAD reducing the vascular light with the help of a small piece PE-10 tube. Ventricular myocytes were isolated from Sham and $\mathrm{I} / \mathrm{R}$ wistar rats by enzymatic dissociation (collagenase type II, Worthington) using Langendorff perfusion. $\left[\mathrm{Ca}^{2+}\right]_{\mathrm{i}}$ transients and $\mathrm{Ca}^{2+}$ sparks were recorded in intact myocytes loaded with fluorescent $\mathrm{Ca}^{2+}$ dye (Fluo-3 AM). Images were obtained with confocal microscopy (Leica TCS SP5).

We observed that $\mathrm{I} / \mathrm{R}$ treatment produced significant decrease in cytosolic $\left([\mathrm{Ca} 2+]\right.$ cyto) and intranuclear $\left([\mathrm{Ca} 2+]_{\text {nuc }}\right) \mathrm{Ca} 2+$ transients in adult cardiomyocytes in the risk zone. Similar alterations in $[\mathrm{Ca} 2+] \mathrm{i}$ transients were observed in the remote zone. I/R 
treatment induces also a reduction in sarcoplasmic reticulum $\mathrm{Ca} 2+$ content in both zones. The analysis of sparks showed that it frequencies decreased in both zones. These $[\mathrm{Ca} 2+] \mathrm{i}$ changes were associated with an increase in several classical hypertrophic genes such as ANF, BNP, SKA and alpha-MHC and with an alteration in the expression of several TRP channels.

The calcium homeostasis undergoes significant changes during $\mathrm{I} / \mathrm{R}$, not only in the ischemic area, but also occurs in the remote area. These calcium changes may contribute to the development of cardiac hypertrophy and heart failure.

$\mathrm{Ca} 2+$ homeostasis, Ischemia/Reperfusion, TRP channels

\section{S4-2}

\section{ROLE OF SARAF IN THE REGULATION OF $\mathrm{CA}^{2+}$ ENTRY}

Juan Antonio Rosado Dionisio

Department of Physiology, Cell Physiology Research Group, University of Extremadura, Cáceres, Spain

Receptor-operated $\mathrm{Ca}^{2+}$ entry is a major mechanism for $\mathrm{Ca}^{2+}$ influx that might occur via receptor-activated channels, second messenger-operated channels or store-operated channels. The latter consist either of Orail subunits, forming $\mathrm{Ca}^{2+}$ release-activated $\mathrm{Ca}^{2+}$ (CRAC) channels, or by Orai1 and TRPC1 subunits, which form cation permeable store-operated $\mathrm{Ca}^{2+}$ (SOC) channels (Desai et al., 2015). CRAC and SOC channels are activated by the intraluminal $\mathrm{Ca}^{2+}$ sensor STIM1 and its homolog STIM2 upon discharge of the intracellular $\mathrm{Ca}^{2+}$ stores.

A new STIM regulatory protein has been identified (Palty et al., 2012). The store-operated $\mathrm{Ca}^{2+}$ entry (SOCE)-associated regulatory factor (SARAF) is a 339 amino acid endoplasmic reticulum (ER)-resident protein that interacts with STIM to facilitate slow $\mathrm{Ca}^{2+}$-dependent inactivation of SOCE, prevent STIM1 spontaneous activation and induce STIM1 deoligomerization upon $\mathrm{Ca}^{2+}$ store refilling. Furthermore, the interaction of SARAF with STIM proteins has been suggested to modulate both cytosolic and ER $\mathrm{Ca}^{2+}$ concentrations (Palty et al., 2012). In addition, we have recently reported that SARAF is able to interact to and modulate Orail and TRPC1 function independently of STIM1. Using proximity ligation and co-immunoprecipitation assays we have found a dynamic and reciprocal interaction of SARAF with STIM1 and Orail, so that $\mathrm{Ca}^{2+}$ store discharge leads to SARAF dissociation of STIM1 reaching a minimal association about $30 \mathrm{~s}$ after store depletion, which paralleled the increase in SARAF-Orail interaction, followed by reinteraction of SARAF with STIM1 and dissociation of Orail (Albarran et al., 2016a). As determined using different Orail constructs, SARAF interacts with the C-terminal region of Orail and, in cells with a low STIM1 expression, enhances the activation of the channel (Albarran et al., 2016a). By contrast, SARAF is a negative modulator of TRPC1 channel function that is able to interact with this channel in the absence and presence of STIM1.

Finally, using siRNA-based and overexpression approaches we have reported that SARAF negatively regulates the arachidonateregulated $\mathrm{Ca}^{2+}$ (ARC) channels (Albarran et al., 2016b), consisting of Orai1 and Orai3 subunits and plasma membrane (PM)-resident
STIM1. We have reported the expression of SARAF in the ER, as well as in the PM, where this protein interacts with Orai proteins. Summarizing, SARAF is a key regulatory protein that modulates receptor-operated $\mathrm{Ca}^{2+}$ entry, including SOCE and $\mathrm{Ca}^{2+}$ influx via ARC channels.

Supported by MINECO (BFU2013-45564-C2-1-P) and Junta de Extremadura-FEDER (GR15029).

Albarran L, Lopez JJ, Amor NB, Martin-Cano FE, Berna-Erro A, Smani T, Salido GM, Rosado JA (2016a) Dynamic interaction of SARAF with STIM1 and Orai1 to modulate store-operated calcium entry. Sci Rep 6: 24452

Albarran L, Lopez JJ, Woodard GE, Salido GM, Rosado JA (2016b) Store-operated Ca2+ entry-associated regulatory factor (SARAF) plays an important role in the regulation of arachidonate-regulated Ca2+ (ARC) channels. J Biol Chem 291: 6982-6988

Desai PN, Zhang X, Wu S, Janoshazi A, Bolimuntha S, Putney JW, Trebak M (2015) Multiple types of calcium channels arising from alternative translation initiation of the Orail message. Science signaling 8: ra74

Palty R, Raveh A, Kaminsky I, Meller R, Reuveny E (2012) SARAF inactivates the store operated calcium entry machinery to prevent excess calcium refilling. Cell 149: 425-438

SARAF, Store-operated calcium entry, ARC channels, Orail, STIM1

\section{S4-3}

FUNCTIONAL COUPLING OF GABA A/B $_{\text {B }}$ RECEPTORS AND TRPV4 CHANNEL MEDIATES RAPID PROGESTERONE SIGNALING IN THE OVIDUCT

Miguel Angel Valverde

Universidad Pompeu Fabra, Barcelona, Spain

Progesterone (P4) modulates the transport of gametes and embryos in the oviduct through the regulation of ciliary beat frequency $(\mathrm{CBF})$ and/or peristalsis. Medium- to long-term exposure to $\mathrm{P} 4$ reduces $\mathrm{CBF}$ through a still largely unknown mechanism. We now report a close functional interaction between the TRPV4 cationic channel and the $\mathrm{GABA}_{\mathrm{A} / \mathrm{B} 1}$ receptor complex to promote rapid responses to $\mathrm{P} 4$ characterized by oscillatory $\mathrm{Ca}^{2+}$ signals and increased $\mathrm{CBF}$ in oviductal ciliated cells. This rapid response was insensitive to classical and membrane $\mathrm{P} 4$ receptors inhibition but was prevented by inhibition of TRPV4, GABA $\mathrm{A}_{\mathrm{B}}, \mathrm{G}_{\mathrm{i} / \mathrm{o}}$, phospholipase $\mathrm{C}$, inositol trisphosphate receptor $\left(\mathrm{IP}_{3} \mathrm{R}\right)$ or genetic ablation of TRPV4. Analysis of the $\mathrm{Ca}^{2+}$ signals under selective pharmacological inhibition of $\mathrm{GABA}_{\mathrm{A} / \mathrm{B}}$ receptors indicated that activation of $\mathrm{GABA}_{\mathrm{A}}$ is upstream of $\mathrm{GABA}_{\mathrm{B}}$. Besides, the use of fluorescence and bioluminescence resonance energy transfer techniques in heterologous expression systems demonstrated the close association of $\mathrm{GABA}_{\mathrm{A}}$ with $\mathrm{GABA}_{\mathrm{B} 1}$ receptor and the activation of $\mathrm{G}_{\mathrm{i} / \mathrm{o}}$ proteins in response to $\mathrm{P} 4$ and GABA agonists. P4mediated $\mathrm{Ca}^{2+}$ signals were also recreated in HEK293-T cells overexpressing $\mathrm{GABA}_{\mathrm{A} / \mathrm{B}}$ and TRPV4-WT but not in cells expressing a TRPV4 lacking the $\mathrm{IP}_{3} \mathrm{R}$ binding site. We propose that 
basal TRPV4-mediated $\mathrm{Ca}^{2+}$ entry in close proximity to $\mathrm{IP}_{3} \mathrm{R}$ is required to initiate and maintain the $\mathrm{Ca}^{2+}$ oscillations triggered by $\mathrm{P} 4$ binding to $\mathrm{GABA}_{\mathrm{A}}$ and transactivation of $\mathrm{G}_{\mathrm{i} / \mathrm{o}}$ protein-coupled $\mathrm{GABA}_{\mathrm{B}}$ receptors. In summary, short-term exposure to $\mathrm{P} 4$ induced rapid $\mathrm{Ca}^{2+}$ oscillations and accelerated $\mathrm{CBF}$ via a mechanism involving $\mathrm{GABA}_{\mathrm{A} / \mathrm{B}}$ receptors, while long-term $\mathrm{P} 4$ exposure reduced $\mathrm{CBF}$ via a classical $\mathrm{P} 4$ receptor.

TRPV4, piezo, calcium, cilium

\section{S4-4}

\section{$\mathrm{CA}^{2+}$ CHANNEL REMODELING IN COLON CANCER}

\section{Carlos Villalobos}

Instituto de Biología y Genética Molecular (IBGM). Consejo Superior de Investigaciones Científicas (CSIC) y Universidad de Valladolid, Valladolid, Spain

$\mathrm{Ca}^{2+}$ is involved in critical cancer processes including proliferation, invasion and survival. Recently, interest is emerging on $\mathrm{Ca}^{2+}$ channel remodeling in cancer. Nevertheless, the molecular and functional mechanisms of intracellular $\mathrm{Ca}^{2+}$ remodeling in colon cancer are unknown. We have investigated the molecular basis of intracellular $\mathrm{Ca}^{2+}$ handling in human colon adenocarcinoma cells and its contribution to cancer features. Fluorescence $\mathrm{Ca}^{2+}$ imaging, patch-clamp electrophysiology, conventional and quantitative PCR, western-blotting, gene silencing and cell proliferation and survival assays were employed for this purpose. We found that storeoperated $\mathrm{Ca}^{2+}$ entry (SOCE), $\mathrm{Ca}^{2+}$ release and $\mathrm{Ca}^{2+}$ entry induced by agonists, and $\mathrm{Ca}^{2+}$ release-activated $\mathrm{Ca}^{2+}$ current ( $\left.\mathrm{I}_{\text {CRAC }}\right)$ are larger in human adenocarcinoma colon cells (HT29) compared to normal human colonic mucosa cells (NCM460). Furthermore, tumor cells display a nonselective store-operated current $\left(\mathrm{I}_{\mathrm{SOC}}\right)$ that is missing in normal cells. In addition, $\mathrm{Ca}^{2+}$ stores are partially depleted in tumor cells relative to normal cells. Enhanced SOCE and depleted $\mathrm{Ca}^{2+}$ stores correlate with increased proliferation and survival characteristic of tumor cells. Moreover, tumor cells display enhanced expression of TRPC1, TRPC4, ORAI1, ORAI2 and ORAI3, and loss of expression of TRPC7, TRPV6 y TRPM8. In addition, STIM2 protein expression is decreased in tumor cells compared to normal cells. Gene silencing experiments show that changes in SOCE and $\mathrm{I}_{\mathrm{CRAC}}$ could be mediated by differences in ORAI1 expression whilst the change in $\mathrm{I}_{\mathrm{SOC}}$ could be mediated by enhanced expression of TRPC1. In addition, $\mathrm{Ca}^{2+}$ store depletion and acquisition of cell death resistance is mediated, at least in part, by changes in the ratio STIM1 to STIM2. We conclude that colon cancer cells undergo a profound intracellular $\mathrm{Ca}^{2+}$ channel remodeling that contributes to cancer hallmarks.

This work was supported by grants BFU2012-37146, BFU201570131R and BIO/VA46/14 from Ministerio de Economía y Competitividad, Spain and Junta de Castilla y León, Spain.

Store-operated calcium entry, colon cancer, calcium, TRPC1, Stim2
S5-1

GASES AND ION CHANNELS - VITAL INTERACTIONS IN CHEMORECEPTION AND BEYOND

Chris Peers

University of Leeds, Leeds, United Kingdom

Gasotransmitters are endogenous, enzymatically generated gases which are increasingly recognised as important signalling molecules. Ion channels are a large family of target proteins which are modulated by gasotransmitters, either directly (e.g. via Snitrosylation by $\mathrm{NO}$ or S-sulfhydration by $\mathrm{H}_{2} \mathrm{~S}$ ) or indirectly (e.g. via altered generation of reactive oxygen species or guanylyl cyclase activation by $\mathrm{CO}$ ), and the consequences of such modulation are physiologically important (see e.g. (Peers et al., 2015; Peers et al., 2012). Within the carotid body, enzymes generating $\mathrm{NO}, \mathrm{CO}$ and $\mathrm{H}_{2} \mathrm{~S}$ have been identified, and these gases exert important influences on carotid body chemoreception. NO and $\mathrm{CO}$ are considered inhibitory, whilst evidence suggests $\mathrm{H}_{2} \mathrm{~S}$ is excitatory, and these effects are at least in part mediated by modulation of ion channels (reviewed in (Prabhakar \& Peers, 2014)). For example, in normoxia, CO derived from heme oxygenase- 2 activates the $\mathrm{O}_{2}$-sensitive maxi-K channel but under hypoxic conditions $\mathrm{CO}$ formation is reduced, channel activity declines and this leads to excitation of the organ (Williams et al., 2004). CO also suppresses $\mathrm{H}_{2} \mathrm{~S}$ generation by cystathionine glyase in normoxia, but in hypoxia this inhibition is relieved, and the elevated $\mathrm{H}_{2} \mathrm{~S}$ levels cause depolarization of glomus cells and carotid body excitation possibly via inhibition of TASK-like $\mathrm{K}^{+}$ channels (Buckler, 2012).

In addition to these important influences of gasotransmitters on carotid body chemotransduction, modulation of ion channels is of physiological and pathological importance in numerous other tissues. For example, $\mathrm{CO}$ inhibition of the delayed rectifier $\mathrm{K}^{+}$channel Kv2.1 provides protection of neurons (Dallas et al., 2011) and certain cancers (Al-Owais et al., 2012) from apoptosis. Both CO and $\mathrm{H}_{2} \mathrm{~S}$ inhibit T-type $\mathrm{Ca}^{2+}$ channels, and in the case of $\mathrm{CO}$ this suppresses vascular smooth muscle proliferation (Duckles et al., 2015; Elies et al., 2014). Of pathological importance is the effect of $\mathrm{CO}$ in the heart; $\mathrm{CO}$, acting via formation of $\mathrm{NO}$, increases the late $\mathrm{Na}^{+}$current in cardiac myocytes, thereby inducing early afterdepolarization type (LQT-3-like) arrhythmias (Dallas et al., 2012). As our understanding of the (patho)physiological importance of gasotransmitters continues to develop, the potential to use these gases as therapeutic interventions also grows. However, our understanding remains incomplete, and further evaluation of gasotransmitter targets - including ion channels - is required before we can exploit their actions for clinical benefit.

Supported by the British Heart Foundation, the MRC, Alzheimer's Research UK and the Alzheimer's Society.

\section{References}

Al-Owais,M.M. et al. (2012). CO mediates the anti-apoptotic effects of heme oxygenase- 1 in medulloblastoma DAOY cells via $\mathrm{K}+$ channel inhibition. J Biol Chem 287, 24754-24764.

Buckler,K.J. (2012). Effects of exogenous hydrogen sulphide on calcium signalling, background (TASK) K channel activity and 
mitochondrial function in chemoreceptor cells. Pflugers Arch 463, 743-754.

Dallas,M.L. et al. (2011). Carbon monoxide protects against oxidant-induced apoptosis via inhibition of Kv2.1. FASEB J 25, 1519-1530.

Dallas,M.L. et al. (2012). Carbon Monoxide Induces Cardiac Arrhythmia via Induction of the Late $\mathrm{Na}+$ Current. Am J Respir Crit Care Med 186, 648-656.

Duckles,H. et al. (2015). Heme oxygenase-1 regulates cell proliferation via carbon monoxide-mediated inhibition of T-type Ca2+ channels. Pflugers Arch 467, 415-427.

Elies,J. et al. (2014). Hydrogen sulfide inhibits Cav3.2 T-type Ca2+ channels. FASEB J 28, 5376-5387.

Peers,C. et al. (2012). Modulation of ion channels by hydrogen sulfide. Antioxid Redox Signal 17, 95-105.

Peers,C. et al. (2015). Diverse mechanisms underlying the regulation of ion channels by carbon monoxide. Br J Pharmacol 172, 1546-1556.

Prabhakar,N.R. \& Peers,C. (2014). Gasotransmitter regulation of ion channels: a key step in o2 sensing by the carotid body. Physiology (Bethesda) 29, 49-57.

Williams,S.E. et al. (2004). Hemoxygenase-2 is an Oxygen Sensor for a Calcium-Sensitive Potassium Channel. Science 306, 20932097.

Gasotransmitter, ion channel, carbon monoxide, hydrogen sulfide, nitric oxide

\section{S5-2}

FROM CAROTID BODY OXYGEN SENSING TO CHRONIC INTERMITTENT HYPOXIA EFFECTS ON SPONTANEOUS TUMORIGENESIS. THE JOURNEY OF CONSTANCIO'S GROUP

M.teresa Gallego-Martin (1), M.asuncion Rocher ${ }^{(1)}$, M Teresa Agapito ${ }^{(1)}$, Angela Gomez-Niño ${ }^{(1)}$, Ricardo Rigual ${ }^{(1)}$, Sara Yubero ${ }^{(1)}$, Elvira Gonzalez-Obeso ${ }^{(2)}$, Elena Olea ${ }^{(1)}$, Inmaculada Docio $^{(1)}$, Ana Obeso ${ }^{(1)}$

(1) Facultad de Medicina. IBGM, Universidad de Valladolid-CSIC. CIBER de Enfermedades Respiratorias, ICiii., Valladolid, Spain; ${ }^{(2)}$ Hospital Clinico Universitario, Valladolid, Spain

The carotid body $(\mathrm{CB})$ is a singular organ which senses variations of the arterial blood $\mathrm{PO}_{2}, \mathrm{PCO}_{2}$ and $\left[\mathrm{H}^{+}\right]$. In the middle of 1980 s, based on our experimental data, our laboratory proposed the membrane hypothesis of acute hypoxic chemoreception formulating that the chemoreceptor cells decrease in arterial $\mathrm{PO}_{2}$ is detected by an oxygen sensor, and the reduction in the opening probability of $\mathrm{O}_{2}$-sensitive $\mathrm{K}^{+}$channels (Lopez-Barneo et al., 1988) depolarizes chemoreceptor cells. Activation of voltage dependent $\mathrm{Na}^{+}$and $\mathrm{Ca}^{2+}$ channels increases intracellular free $\left[\mathrm{Ca}^{2+}\right]$ promoting release of catecholamines and other neurotransmitters, which augment the activity of the carotid sinus nerve producing hyperventilation (Gonzalez et al., 1994). Second messengers, such as cAMP, EPAC, and hydrogen sulphide, are capable to modulate the flow of information in the chemoreception transduction cascade (Rocher et al., 2009; Gallego-Martin et al., 2015). Nowadays, oxygen sensor identity remains unknown.
Physiological systemic effects of $\mathrm{CB}$ activation have long been studied. In recent years, CB involvement in several pathological processes turns into a field of high interest. Some of these pathological processes are respiratory diseases that involve hypoxic situations, such as chronic sustained hypoxia in chronic obstructive pulmonary disease and chronic intermittent hypoxia $(\mathrm{CIH})$ in obstructive sleep apnea (OSA). As a consequence of $\mathrm{CIH}$, repetitive $\mathrm{CB}$ activation produces sympathetic overstimulation and redox imbalance, with high levels of reactive oxygen species (Agapito et al., 2009). CB overexcitation in OSA patients could be related to the appearance of cardiovascular pathologies (endotelial dysfunctions, hypertension, cardio- and cerebrovascular accidents), hepato-metabolic alterations (obesity, insulin resistance, non-alcoholic hepatic steatosis), and neuropsychiatric diseases (anxiety, depression, dementia).

Recently, it has been proposed a relationship between $\mathrm{CIH}$, the main constitutive element of OSA, and cancer. Limited studies have evidenced that $\mathrm{CIH}$ augments growth and metastasis rate of implanted tumors in mice (Almendros et al. 2013). In OSA patients, although with some discrepancies, an association between OSA and cancer incidence/mortality has been reported (Nieto et al., 2012). Discrepancies could be due to the large number of OSA-linked co-morbidities. Trying to simplify this complex pathological human situation, $\mathrm{CIH}$ as a single variable has been used to evaluate its effects on spontaneous tumorigenesis. In an old outbreed murine model, two intensities of $\mathrm{CIH}$ were applied (12\% $\mathrm{O}_{2}$, moderate, and $7.5 \% \mathrm{O}_{2}$, severe) mimicking two stages of OSA patients pathological situation. We have observed that long term (3 months) severe $\mathrm{CIH}$ augments spontaneous lung tumor incidence. These tumors are lung typical carcinoids, a type of neuroendocrine tumor. These findings could help to interpret cancer incidence in OSA patients and, on the other hand, they alert about the necessity of further designed human studies to evaluate if OSA could augment only specific types of cancer incidence.

Funding: BFU2015-70616-R, MINECO/FEDER, UE; CIBERCB06/ 06/0050, CIBERES; APRO-I Valladolid, Asociación Española Contra el Cáncer

Carotid body, oxygen sensing, intermittent hypoxia, tumorigenesis

\section{S5-3}

\section{CHEMORECEPTION AND METABOLIC DISEASES}

\section{Silvia Conde}

CEDOC, NOVA Medical School, Faculdade de Ciências Médicas, Lisboa, Portugal

Metabolic diseases affect millions of individuals globally, contributing to increasing morbidity and mortality worldwide. This represents an alarming public health problem, with economic and social repercussions, reinforcing the urgency to identify treatment interventions that can effectively stalk this epidemic, particularly in an early phase of the diseases.

The carotid bodies (CB) are peripheral chemoreceptors that classically sense hypoxia, hypercapnia and acidosis, responding through an increase in action potential frequency in their sensory 
nerve, the carotid sinus nerve (CSN). CSN activity is integrated in the brainstem to induce respiratory responses aimed, primarily, to normalize blood gases, via hyperventilation (Gonzalez et al., 1994), and to regulate blood pressure and cardiac performance, via sympathetic nervous system activation (Marshall, 1994). In the last decade the $\mathrm{CB}$ has been described as a major metabolic sensor implicated in the control of energy homeostasis (Koyama et al., 2000; Wehrwein et al., 2010). In the same line of evidence, we have recently described that carotid body (CB) overactivation is involved in the etiology of insulin resistance (IR) and arterial hypertension (HT) in animal models of metabolic syndrome (Ribeiro et al., 2013). Additionally, we have demonstrated that $\mathrm{CB}$ activity is increased in IR animal models, and that carotid sinus nerve (CSN) resection prevents the development of IR and HT induced by hypercaloric diets (Ribeiro et al., 2013). This preclinical work is also supported by clinical data: type 2 diabetes patients submitted to hyperbaric oxygen therapy, that functionally suppresses $\mathrm{CB}$ activity, showed an ameliorated glucose tolerance and a significant reduction of plasma glucose levels (Peleg et al., 2013; Vera-Cruz et al., 2015). In the present talk, new data demonstrating that the abolishment of CB activity displays therapeutic potential in pre-existing metabolic diseases will be showed as well as the mechanisms of CB-mediated pathogenesis of metabolic diseases. We have observed that chronic bilateral CSN denervation fully restored insulin sensitivity, fasting plasma glucose, fasting plasma insulin, blood pressure and lipid profile to physiological levels, also leading to a restoration of insulin signalling pathways in skeletal muscle and adipose tissue in hypercaloric diet rats. Moroever, CSN denervation improved glucose uptake by the liver and by the mesenteric adipose tissue concomitantly with the restoration of the autonomic imbalance in these animals. Another important fact was that the metabolic improvement induced by CSN surgical ablation was unremitting throughout time, even in animals continuously submitted to hypercaloric diets. Altogether, the data generated shed light on the mechanisms of CB-mediated pathogenesis of metabolic diseases and show that CSN denervation restores glucose and lipid homeostasis establishing a sustained and effective intervention for metabolic diseases.

Carotid body, insulin action, glucose homeostasis

\section{S5-4}

CONTRIBUTION OF CAROTID BODY CHEMORECEPTORS TO THE HYPERTENSION AND AUTONOMIC CHANGES INDUCED BY INTERMITTENT HYPOXIA IN AN EXPERIMENTAL MODEL OF OBSTRUCTIVE SLEEP APNEA

\section{Rodrigo Iturriaga}

Pontificia Universidad Católica de Chile, Santiago, Chile

The carotid body (CB) is the main peripheral chemoreceptor that senses arterial $\mathrm{PO} 2, \mathrm{PCO} 2$ and $\mathrm{pH}$. The current paradigm considers the $\mathrm{CB}$ as the main peripheral hypoxic sensor, which initiates the reflex cardiorespiratory adjustments to hypoxia and mediates the ventilatory acclimation to high altitude. However, a growing body of new evidence supports the novel idea that an abnormally enhanced $\mathrm{CB}$ chemosensory input to the central nervous system contributes to sympathetic hyperactivation and consequent cardio-metabolic pathologies. The obstructive sleep apnea (OSA) syndrome, a growing worldwide sleep-breathing disorder is recognized as an independent risk factor for hypertension. OSA is characterized by repeated episodes of complete or partial airflow detention during sleep. Among the disturbances produced by OSA, the chronic intermittent hypoxia $(\mathrm{CIH})$ is considered the main factor for the development and progression of the hypertension. Although the link between OSA and hypertension is well established, the pathogenic mechanisms responsible for the hypertension are not entirely understood. It has been proposed that $\mathrm{CIH}$ produces oxidative stress, inflammation, and sympathetic hyperactivity, which led to endothelial dysfunction and hypertension. However, recent experimental evidence supports the proposal that the CB plays a pivotal role in the development of the hypertension. Indeed, $\mathrm{CIH}$ selectively enhances the $\mathrm{CB}$ chemosensory discharges in normoxia and hypoxia, which in turn potentiates the sympathetic outflow to the arterial blood vessels.

We studied the role of oxidative stress on the enhanced $\mathrm{CB}$ chemosensory responses to hypoxia and the hypertension in male Sprague Dawley rats exposed to $\mathrm{CIH}(5 \% \mathrm{O} 2,12$ times $/ \mathrm{h} 8 \mathrm{~h} /$ day $)$ for 21 days. We found that ascorbic acid treatment during $\mathrm{CIH}$ reduced the increased systemic and local $\mathrm{CB}$ oxidative stress, the potentiation of $\mathrm{CB}$ chemosensory responses and prevents the hypertension. Taken together the available evidences strongly suggests that oxidative stress is the key mediator of the enhanced $\mathrm{CB}$ chemosensory responses to hypoxia and the hypertension induced by $\mathrm{CIH}$, but the actions of oxidative stress on the arterial blood pressure regulation in humans and animals exposed to $\mathrm{CIH}$ may occur in multiple sites of the chemoreflex pathway, including cardiovascular brainstem nuclei and arterial vessels.

To assess the contribution of the $\mathrm{CB}$ to the hypertension and autonomic changes induced by $\mathrm{CIH}$, the $\mathrm{CBs}$ were ablated once rats became hypertensive following $\mathrm{CIH}$. Male Sprague Dawley rats $(250 \mathrm{~g})$ were exposed to sham conditions for 7 days and then to $\mathrm{CIH}$ for 28 days. At 21 days of $\mathrm{CIH}$ exposure, both $\mathrm{CBs}$ were ablated (CBA) and rats were exposed for 7 additional days to CIH. Arterial blood pressure (BP) and ventilatory chemoreflex response to hypoxia (VHR) were measured in conscious rats by radio-telemetry and plethysmography, respectively. In addition, cardiac autonomic balance, cardiac baroreflex gain and arrhythmia score was assessed during the experiments. $\mathrm{CIH}$ induced hypertension (Mean BP $\sim 10 \mathrm{mmHg}$ in 3-4 days), enhanced VHR, induced autonomic imbalance towards sympathetic preponderance, reduced cardiac baroreflex gain, and increased the number of arrhythmias. CBA promptly normalized BP, reduced VHR and restored cardiac autonomic and baroreflex function, and reduced the number of arrhythmias. Systemic oxidative stress was unaffected by CBA. Present results show that autonomic alterations induced by $\mathrm{CIH}$ depend on the enhanced $\mathrm{CB}$ responsiveness support a main role for the $\mathrm{CB}$ in the $\mathrm{CIH}$ induced hypertension in OSA patients.

\section{Supported by FONDECYT 1150040}

Autonomic dysfunction, carotid body, intermittent hypoxia, hyperetnsion, sleep apnea 
S6-1

STRENUOUS ENDURANCE EXERCISE: CARDIOHEALTHY OR 'CARDIOTOXIC'?

\section{Helios Pereja-Galeano}

Instituto de Investigación Hospital 12 de Octubre ('i + 12’), Madrid. Universidad Europea Madrid, Madrid, Spain

The health benefits of regular physical activity are irrefutable (particularly, regarding a reduced risk of cardiovascular morbidity and mortality). Yet a contrary belief is the prevailing one (since the start of last century) regarding elite (or professional) sports. In fact, some authors have introduced the concept of 'over exercise', where performing strenuous endurance activities (such as running) for more than $1 \mathrm{~h}$ /day could increase the risk of 'cardiotoxicity', particularly in the form of atrial fibrillation. Yet, if existing, the 'cardiotoxic' effects of strenuous endurance exercise only affect a really small percentage of the population. The real problem is actually 'under exercise', with approximately $1 / 3$ of adults worldwide being currently inactive, that is, not even engaging in $150 \mathrm{~min}$ of moderate physical activity (such as brisk walking) per week. Our intent is to provide an unbiased, balanced overview of the cardiovascular effects of strenuous endurance exercise.

\section{S6-2}

\section{NO EVIDENCE OF ADVERSE CARDIAC REMODELING IN FORMER ELITE ENDURANCE ATHLETES}

\section{Enrique Serrano Ostáriz}

Universidad de Zaragoza, Zaragoza, Spain

Low to moderate intensity aerobic or endurance exercise (eg, brisk walking daily for 30-60 min) has well-documented beneficial impacts on cardiovascular morbidity and mortality.

Whilst many people follow this type of exercise prescription, a sizeable number of amateur "athletes" engage in strenuous training and competitive activity whose exercise intensity and duration clearly exceed the standards programs established by various organizations for healthy physical activity. Whether the elevated exercise volume completed by amateur "athletes" provides additional health benefits or, controversially, increased health risk is currently not clear.

An increasing number of studies have shown that prolonged strenuous exercise promotes the elevation of cardiac biomarkers. Post-exercise values for serum troponins (cTnI and cTnT) and N-terminal pro BNP (NT-proBNP), which are accepted parameters for the identification of cardiac damage and dysfunction, are often higher than the population upper reference limit (URL) in a high percentage of athletes in these strenuous competitions. Although the clinical significance of the increase in cardiac-specific biomarkers after strenuous endurance exercise in healthy athletes is unclear, it is of interest to determine the importance and role of factors that may promote biomarker release.
The debate about the cardiovascular health consequences of strenuous exercise completed over many years of training and competition has largely arisen because of the common observation of elevated cardiac biomarkers after competitive prolonged endurance exercise. However, the effects of much higher exercise loads (eg, training for and competing in marathon running events) on a previously healthy heart remain controversial, and some authors warn of the potentially deleterious cardiac effects of long-term strenuous endurance exercise (6-9). The concept of 'cardiac overuse injury' (or 'over exercise') has been recently reported to group the potential negative effects of strenuous endurance exercise.

Middle-aged/older athletes with a lifelong history of training/ competition at the highest possible level (i.e., international) are the paradigm of humans whose hearts are most likely to suffer the potential deleterious effects of long-term endurance exercise. Veteran endurance athletes who have performed regular strenuous endurance exercise over more than 30 years show a cardiac remodeling pattern characterized by larger LV, RV and LA cavities compared with non-athletic healthy controls, with the greater LV size matched by an increased LV myocardial mass and with no evidence of permanent major cardiac damage or fibrosis assessed by imaging or blood biomarkers. At least in resting conditions (ie, with no prior bout of exhaustive exercise), both RV systolic function and cardiac biomarkers seem unaffected by such an athletic lifestyle, corroborating the notion that any alterations detected in these variables are usually transient in nature.

Endurance exercise, cardiac remodeling, cardiac fibrosis.

S6-3

\section{SUDDEN CARDIAC DEATH IN PROFESSIONAL CYCLING}

Alejandro Santos Lozano

Universidad Europea Miguel de Cervantes (UEMC), Valladolid, Spain

The popularity of long-term strenuous activities has contributed to an increase in sudden cardiac deaths during the last decade, intensifying the debate about security in such events. One of the paradigms of long-term strenuous activity is the professional cycling, especially the Tour of France, where ciclists accumulate more than $4 \mathrm{~h}$ of intense activity for 3 weeks, sometimes under extreme environmental conditions. After analyzing the number of deaths in the history of men's professional cycling during competition, the incidence of sudden death was defined in the Tour of France as 0.007:100,000 participant-years and 0 participant-years for cyclists medically evaluated before the competition. Likewise, there was an incidence of sudden death of 0 participant-years in professional competitions from 2009 to 2015. After analyzing the data of sudden cardiac death in professional cyclists during competition, this seems to be a very rare phenomenon, even showing a lower incidence than other professional sports.

Health, sports, cardiovascular events 
S7-1

\section{DEVELOPING PRACTICAL PHYSIOLOGY WITH ARDUINO}

\author{
Guillermo Álvarez De Toledo, Guillermo Álvarez De Toledo \\ Tabares
}

Facultad de Medicina. Universidad de Sevilla, Sevilla, Spain

Practical teaching of Physiology is fundamental and a must for the optimal in-depth assimilation of physiological knowledge by students. Practical teaching provides a step by step learning process, leading to a solid understanding of key physiological concepts, and how physiological variables are measured with instruments. Traditionally, the equipment required for practical teaching of physiology has not been cheap, on the contrary, either lab equipment itself, or lab adapted apparatus has been used to teach practical physiology. The development of open source and inexpensive platforms, such as Arduino, offers the opportunity to create hardware and software applications oriented towards Physiology teaching, making almost a quasi personalized set up of physiology experiments. Particularly, the Arduino M0 Pro, an advanced version of the popular Arduino UNO, provides a microcontroller with reasonable speed (48 MHz), 6 analog inputs (12 bit), 1 DAC (10 bit) and many programmable I/O digital pins, which are suitable for the acquisition of properly, conditioned physiological variables. Careful design of data acquisition subroutines in Arduino, can match, reasonably, standards of professional level interfaces. Basically, this microcontroller is as powerful as computer interfaces were 25 years ago, with a price of around $30 €$. We have created circuits to connect different pressure (high and low), airflow, thermistors, microphone (stethoscope) and humidity sensors with Arduino to monitor blood and respiratory pressures, airflow, temperature, heart sounds or electrical skin resistance. Also, we have developed a pulse oximeter and several voltage followers and amplifiers to measure ECG, EEG, EMG, or EOG. The software controlling the acquisition of physiological variables with Arduino was written in Java, and provides a step by step guide for each experiment described in this platform. This platform is easy to adapt to any new experiment on demand.

Arduino, practical physiology, sensors, blood pressure, ECG, EEG

S7-2

\section{CONTINUOUS ASSESSMENT IN PHYSIOLOGY}

Jordi Palés

Facultad de Medicina y Ciencias de la Salud, Universidad de Barcelona, Barcelona, Spain

One of the main challenges of the Bologna reform is to implement the paradigm of outcome-based medical education that implies the assessment of these outcomes. The acquisition of learning outcomes along a training program is an on-going process and therefore checking whether the learning outcomes are achieved must also be progressive. Continuous assessment is based on this concept.
Continuous assessment implies to develop throughout the course different assessment activities, by using several tools and performing multiple measurements at different times of the training process. In this sense it is often said that an individual measure is not a measure, and the experts in medical education advocate for the need to have different inputs of student performance at different times obtained by some assessment tools to have a clear idea of the progress of the students.

A continuous assessment system has advantages for both students and teachers. By a continuous assessment, students assimilate knowledge and acquire the intended learning outcomes gradually. Moreover, continuous assessment with a strong formative component throughout the course, allows students to receive feedback on their strengths and weaknesses, about their own learning pace and gives opportunities to correct their mistakes, offering remedial possibilities and the reorientation their learning if it is necessary. Thus we are moving from the paradigm of the assessment of learning to assessment for learning. Continuous assessment also largely eliminates anxiety afflicting the students when they have to face a final exam, facilitates continuous learning and not intensive at the end of the process. A wellplanned continuous assessment with clear goals, well-used tools and a logical temporal sequence can make unnecessary the final exam. Good planning requires also a correct description of the activities that will be evaluated, instruments to be used and especially the evaluation criteria to be followed. Moreover it has been reported that a system of continuous assessment improves student performance and the number of these that succeed in the course.

For teachers, undoubtedly continuous assessment represents more work but certainly it allows them to check in a more reliably manner the student's learning, provides information on the acquisition of the intended learning outcomes and gives a feed-back on the quality of the own teaching and the quality of the training program.

Continuous assessment can use different tools and activities. Among them we can consider practical approach of cases, multiple choice questions, case discussion in teamwork, problem based learning, oral presentations of topics or case studies, technical skills, development of portfolios, etc. The use of online resources that allow immediate interaction between teachers and student's audience and immediate feedback are very useful in continuous assessment.

All these methods and activities are suitable for use in different disciplines and logically Physiology. In the presentation we will describe the system used in the course of Medical Physiology at the Faculty of Medicine of the University of Barcelona.

Assessment, Continuous Assessment, outcome-based learning

TOOLS FOR E-LEARNING/TEACHING (E-L/T) OF PHYSIOLOGY

Emilio J. Sánchez Barceló, Javier De La Hera Fernández, Paula Hoz San Bartolomé, Dolores Mesiavilla Aguado

Universidad de Cantabria, Santander, Spain 
The technologies of information and communication (TICs) have drastically changed the retrieval of scientific information in only a couple of decades. What the TICs have given us is virtually an unlimited access to global sources of knowledge and empowerment of autonomous or remote training.

The so-called "e-learning" is gaining relevance in the context of the general methods for learning/teaching and the L/T of physiology is not the exception. At present, in most of our universities, the "classical methods" based on the "magisterial class" as the pivotal teaching activity is still coexisting with an incipient development of e-L/T. The increase in the use of $\mathrm{e}-\mathrm{L} / \mathrm{T}$ in our universities will depend on factors such as: a) the formation of teachers in TICs; b) the presence, in the university departments, of TICs technicians as part of the nonteaching staff; c) the universal access to internet and TIC technologies in the campus; and, d) improving the English skills of students and teachers

The e-learning requires the use of educational programs and contents through electronic media such as computers, information networks (internet or intranet), smart phones, tablets, media players (MP3, MP4, MP5, etc.), interactive digital television, etc. In summary, a set of tools that let distance learning.

It is important to emphasize that e-L/T does not meant "without teachers"; on the contrary, it means "with many teachers", with a practically unlimited number of professors. E-learning as a training tool in the medical sciences can be combined with other forms of traditional teaching focused on classroom teaching, forming what is known as b-learning (blended learning). The role of the "local" professor will be to guide the students to get information wherever it is, whatever this site; to assess the quality of this information; to understand its meaning; to elaborate global concepts from partial information.

In this presentation, we will review some of the TIC tools used in the field of medical education.

E-learning, physiology,technologies of information and communication (TICs)

\section{S7-4}

CASE STUDIES FOR SMALL GROUP DISCUSSIONS ON MEDICAL PHYSIOLOGY: A COLLECTION ARRANGED BY THE GRUPO INTERUNIVERSITARIO DE INNOVACIÓN DOCENTE EN FISIOLOGÍA (GIDF)

\section{Diego Sanchez Romero}

Universidad de Valladolid, Valladolid, Spain

Alberola A. ${ }^{(7)}$, Arribas S. ${ }^{(1)}$, Álvarez D ${ }^{(4)}$, Conde M.V. ${ }^{(1)}$, Cózar, I. $^{(8)}$, Echevarría E. ${ }^{(5)}$, Escanero J.F. ${ }^{(9)}$, Fernández-Torre J.L. ${ }^{(3)}$, Ganfornina L. ${ }^{\left({ }^{8}\right)}$, Giménez I. ${ }^{(9)}$, Giráldez T. ${ }^{(4)}$, González C. ${ }^{(1)}$, Gual A. (2), Guerra M. ${ }^{(9)}$, Mediavilla M.D. ${ }^{(3)}$, Montoro R.J. ${ }^{(6)}$, Palés J. ${ }^{(2)}$, Pié J. ${ }^{(9)}$, Sánchez D. ${ }^{(8)}$, y Sánchez-Barceló E. ${ }^{(3)}$

Universities: ${ }^{(1)}$ Autónoma de Madrid, ${ }^{(2)}$ Barcelona, ${ }^{(3)}$ Cantabria, ${ }^{(4)}$ La Laguna, ${ }^{(5)}$ País Vasco, ${ }^{(6)}$ Sevilla, ${ }^{(7)}$ Valencia, ${ }^{(8)}$ Valladolid y ${ }^{(9)}$ Zaragoza
The Inter-university Group for Innovative Physiology Teaching (GIDF) has been working over the last decade to educate teachers in methods for more effective teaching of Medical Physiology. This is particularly relevant after the launching of the European Higher Education Area and the Medical Degree in our Universities, whose goal is to train future doctors to provide quality clinical care in the realm of evidence-based Medicine. Because of this importance, the GIDF has been recently recognized by the University of Valladolid as a Multicenter Group in Innovative Teaching.

For the last 4 years, the GIDF has been working on a compendium of Case Studies on Physiology to be used by 2nd year Medical School students in Small Group Discussion sessions. The cases have been designed using a problem-based learning paradigm, and represent real-life situations where students must learn how to identify, think about and use key physiological concepts. Each case is presented by the teacher, followed by a series of sequential questions to be discussed by the students, while allowing them to use printed or web-based information retrieval. The discussions are followed by a knowledge sharing sessions moderated by the teachers.

To achieve our goal, several working groups of 2-4 teachers met to determine key concepts and competences to explore in each physiology system. The cases outlined by each working group were reviewed and homogenized by a different group of teachers. Finally, the case studies were assessed for relevance to student curricula and assess their expected class time.

The final collection is composed of 56 case studies that will be published in 2016 by the Publishing Service of the University of Valladolid. We hope our work will help both students and teachers to achieve a better learning outcome in Medical Physiology in Spanish Universities.

Teaching, Case study, Problem-based learning

S8-1

DIETARY SPRAY-DRIED ANIMAL PLASMA SUPPLEMENTS PREVENT INTESTINAL AND PERIPHERAL INFLAMMATION IN MURINE MODELS

Miquel Moretó, Lluïsa Miró, Anna Pérez-Bosque

Facultad de Farmacia, Universidad de Barcelona, Barcelona, Spain

Epidemiological studies indicate that farm animals supplemented with bovine or porcine plasma proteins have a reduced morbidity and mortality. These effects are especially relevant around weaning, when young animals are exposed to stressful conditions, such as switching to a solid diet and mother separation. During this period, there is activation of the gut-associated lymphoid tissue (GALT) that results in intestinal inflammation and increased intestinal permeability, which contributes to progression of inflammation. Spray-dried plasma (SDP) is a protein-rich product obtained from the industrial fractionation of blood from porcine and bovine animals slaughtered for human consumption. Blood is centrifuged to separate blood cells and spray-dried using high 
pressure and a temperature over $80{ }^{\circ} \mathrm{C}$ for a very short period of time. With this procedure, most biological activity of the proteins is preserved. SDP contains over $90 \%$ protein, more than $50 \%$ of which consists of immunoglobulins, mainly IgG with broad spectrum of specificity against a variety of pathogens and antigens, but doesn't content specific antibodies developed against particular pathogen or antigen. It also contains a complex mixture of functional and biologically active components such as growth factors and cytokines. The effect of SDP supplementation has been studied in two models of inflammation: first, a model of acute induction of a mild intestinal inflammation using the $S$. aureus enterotoxin B (SEB), in rats and mice and, second, a genetic model of spontaneous colitis, using mice lacking the mdr1 gene. Plasma proteins reduce the degree of GALT activation by a mechanism involving modulation of Treg lymphocytes and an attenuation of changes that take place in the pro- / anti-inflammatory cytokine profile. In the colitis model, SDP supplementation reduces the severity of mucosal markers during the onset of colitis, and modulates the immune response of the intestinal mucosa and restores the barrier function of the epithelium, and these effects that are also observed in the SEB-induced acute intestinal inflammation model. These effects of SDP are accompanied by changes in defensins expression, tight-junction permeability and mucosal cytokine production. In both intestinal models, the effects of SDP are mediated by an increased Treg response and enhanced release of antiinflammatory cytokines that contribute to mucosal homeostasis. In peripheral tissues, such as the lung mucosa, dietary SDP enhances anti-inflammatory mediators that control the immune pulmonary response in a model of acute lung inflammation; in the uterine mucosa, SDP improves pregnancy rates by attenuating the production of inflammation and stress mediators in a rat model of pregnancy impairment. These results support the view that the mechanism of action of SDP involves extensive communication between GALT and peripheral lymphoid tissues through the common mucosal immune system.

Supported by TRA2009-0317 from the Spanish Ministry of Science and Innovation and grant RDITCRD07-1-0008 from CIDEM and SGR2014-1221 (Government of Catalonia); APC Europe S.A. (Granollers, Barcelona), EnteraHealth Inc. and APC Inc. (Ankeny, USA) also partially funded these studies.

GALT, inflammation models, spray-dried plasma

\section{S8-2}

INTERACTION BETWEEN INHIBITORY NEURONS AND PACEMAKER FUNCTION COORDINATES MYOGENIC MOTOR PATTERNS IN THE COLON

\section{Noemi Mañé, Marcel Jimenez}

Universitat Autonoma de Barcelona, Bellaterra, Spain

The Enteric Nervous System modulates the contractile state of smooth muscle through inhibitory and excitatory motor neurons. Inhibitory neurotransmitters hyperpolarize and therefore relax the smooth muscle while excitatory motor neurons cause smooth muscle contraction. Furthermore, motor neurons interact with pacemaker cells to enhance or inhibit their activity. The aim of this work is to set forth how we characterised enteric neuromuscular transmission and to hypothesise how neurons cooperate with pacemaker cells to orchestrate different motility patterns.

Inhibitory motor neurons elicit inhibitory junction potentials in smooth muscle that consist of a fast (IJPf) followed by a slow (IJPs) hyperpolarization. This is a general mechanism of nerve mediated relaxation in the gastrointestinal tract that occurs in the human small intestine and colon as well as in laboratory and domestic animals. As an example, the mechanism exists even in the colon of horses where nerve mediated interactions are crucial to understand highly prevalent colonic motor disorders. The IJPf is mediated by ATP or a related purine. The purine receptor subtype was identified by using orthoesteric antagonists of the $\mathrm{P}_{2} \mathrm{Y}_{1}$ receptor such as MRS2179, MRS2279 and MRS2500 which block the IJPf in a concentration dependent manner. Recently we have shown that allosteric blockers, such as BPTU, have a similar effect. Accordingly, non-competitive binding sites can be considered a new pharmacological target to modulate propulsive function. $\mathrm{P}_{2} \mathrm{Y}_{1} \mathrm{KO}$ animals lack IJPf and the corresponding purinergic relaxation which reinforces the role of this receptor in purine inhibitory responses. In contrast, the IJPs is blocked by the NOS inhibitor L-NNA and the Guanylil cyclase inhibitor ODQ revealing a nitrergic nature.

Using different frequencies and voltages of electrical field stimulation we have been able to characterise both electrophysiological and mechanical responses to purine-NO co-transmission. Single pulses mimicking single action potentials progressively increase the amplitude of both the IJPf and IJPs when more inhibitory neurons are recruited with higher voltages. When frequency of stimulation increases the IJPf is exponentially reduced whereas the IJPs exponentially increases. This shows that inhibition of muscular activity is possibly dependent of the firing frequency of inhibitory neurons. ATP is predominantly acting at low firing frequencies whereas $\mathrm{NO}$ is predominant at high frequencies. Using these experimental approaches no other inhibitory neurotransmitters or receptors have been functionally identified.

Interstitial Cells of Cajal (ICC) located near the submucous plexus (ICC-SMP) generate slow wave activity. Slow wave activity generates small rhythmic contractions that are the physiological basis of ripples identified with manometric studies in humans. Slow waves can be slightly hyperpolarized by inhibitory neurons but the rhythm is probably omnipresent causing a constant mixing of content. A second myogenic rhythm probably originated in ICC located near the myenteric plexus (ICC-MP) generates cyclic depolarizations of a lower frequency and higher amplitude. This second rhythm can be strongly modulated by either excitatory (mainly cholinergic) and inhibitory neurons. Interaction between slow waves and cyclic depolarizations can induce a "Wax and wane" pattern where the amplitude of slow waves increase and decrease at the same frequency than cyclic depolarizations. The "wax and wane" pattern can be the origin of segmentation described in humans.

We conclude that myogenic motor patterns are intrinsically present in the gut wall. Enteric neurons modulate this activity. Depending on the level of neurogenic excitation, propulsive contractions, segmentation or mixing occurs. Cholinergic contractions possibly potentiate cyclic depolarizations causing propulsion whereas activation of nitrergic inhibitory neurons inhibits cyclic depolarizations and the predominant motor pattern is mixing. An intermediate state of excitability might be responsible for single motor patterns or segmentation.

ICC, purines, Nitric Oxide, smooth muscle 
S8-3

AGE-RELATED CHANGES IN CONTRACTILITY, CA2+ HOMEOSTASIS, SENESCENCE PATHWAY AND MITOCHONDRIAL DYNAMICS IN HUMAN COLON

$\mathrm{M}^{\mathrm{a}}$ José Pozo ${ }^{(1)}$, Pedro J Camello ${ }^{(1)}$, Cristina Camello (1), Francisco E. Martín-Cano ${ }^{(1)}, \mathrm{M}^{\mathrm{a}}$ Teresa Espín ${ }^{(2)}$, Francisco J De Santos ${ }^{(3)}$

(1) Facultad de Enfermería y Terapia Ocupacional, Cáceres, Spain; (2) Hospital Infanta Cristina, Badajoz, Spain; (3) Hospital San Pedro de Alcántara, Cáceres, Spain

Colonic motility is the result of the orchestrated activation of several cellular types located in the different layers of the colon, similar to the motor activity of other parts of the digestive tract. Compared to colonic studies in laboratory animals, human colon contractility papers are scarce specially reporting longitudinal muscle contractility and studies on the effects of age in colonic function are lacking. The aim of this work is to study the effects of age on circular and longitudinal muscle contraction, calcium homeostasis and mTOR pathways and mitochondrial dynamics.

When comparing the myogenic response between colonic muscle layers, the response to depolarization was significantly higher in the longitudinal layer that also showed smaller $\mathrm{EC}_{50}$ values. Longitudinal muscle was more sensitive to bethanechol-induced responses but the amplitude of the responses did not differ significantly between layers. The response to substance P did not differ between layers. Neurogenic responses induced by EFS (electrical field stimulation) showed different shape responses: while the response in the longitudinal muscle was contractile at all tested frequencies, the same stimuli caused relaxation in the circular muscle followed by a rebound contraction. Circular strips presented a decay in their tension after the EFS, while longitudinal strips did not change, suggesting an inhibitory predominant innervation in the circular layer. The pharmacological dissection revealed that longitudinal muscle is mainly innervated by cholinergicadrenergic terminals. On the contrary, circular muscle innervation seems to be predominantly inhibitory. In NANC conditions, the blockade of NO and ATP in circular muscle revealed a reciprocal innervation between nitrergic and purinergic neurons that was not present in the longitudinal layer. The correlation between the myogenic response and age was positive for all stimuli in the circular muscle but negative in the longitudinal one. On the contrary, neurogenic responses were correlated with age in the longitudinal muscle but not in the circular layer. Regarding the contribution of the different neurotransmitters, no one correlated significantly with age.

$\mathrm{Ca}^{2+}$ responses to depolarization and $\mathrm{ACh}$ in intact tissue from patients 50-85 years old responded to depolarization slow and homogenously while ACh responses were faster and occurred in waves that traveled through the tissue. Regarding correlation with age, neither depolarization nor ACh responses were significantly correlated with age, although depolarization showed a slight decrease. The $\mathrm{Ca} 2+$ responses to the neurotransmitter ACh (an activator of the metabotropic IP3 pathway) and to caffeine (which releases intracellular stored $\mathrm{Ca} 2+$ through RyR receptors) showed a slight negative correlation with age. Piecewise linear regression showed that beyond the age of 65 the responses declined. In keeping with this, both responses were significantly smaller in cells from $65+$ year old patients. A similar pattern was also present in the $\mathrm{Ca} 2+$ influx mediated by voltage-activated channels induced by $\mathrm{KCl}$ depolarization.

mTOR constitutive activity in human colonic muscle was not agedependent but there was a significant positive correlation in the mucosa. The phosphorylation of the kinase substrate 4EBP1 was negatively correlated with age, but there were no changes in the phosphorylation of kinase p70-S6, indicating that the negative effects of mTOR activation in the mucosa did not have deleterious effects. Regarding mitochondria dynamics, aging correlated with increases in the mitochondrial fission pathway (indicated by phosphorylation of Drp1) both in muscle and mucosa, whereas the expression of the nuclear receptor Rev-Erb and the PGC- $1 \alpha$, associated to mitochondria biogenesis, had a negative correlation with age in muscle but did not change in the mucose. Mitochondrial fusion had a negative correlation with age (quantified by mitofusine).

Human colon, contractility, Ca2+ homeostasis, mTOR pathway, mitochondria

\section{S8-4}

INFLUENCE OF THE MICROBIOTA ON THE MOUSE INTESTINAL MOTOR FUNCTION: ROLE OF TOLL LIKE RECEPTORS

\section{Laura Grasa}

Universidad de Zaragoza- Facultad de Veterinaria, Zaragoza, Spain

The intestine hosts a complex ecosystem comprising about 1000 different bacterial species. This gut commensal microbiota is essential for the normal development of the immune system and therefore plays a critical role in the maintenance of gut homeostasis. Alterations in the composition of this microbiota (dysbiosis) may result in immune dysregulation and be a pathogenic component of functional gastrointestinal alterations, such as irritable bowel syndrome. Microbiota could influence gut motor function through three identified mechanisms: (1) through the release of bacterial substances or end-products of bacterial fermentation, (2) via intestinal neuroendocrine factors, and (3) indirectly through the effects of mediators released by the gut immune response.

The interaction between gut microbiota and the host is known to be mediated through pattern recognition receptors (PRRs). Tolllike receptors (TLRs) are PRRs that recognize microbial molecules, called microbial-associated molecular patterns (MAMPs), and play a key role in innate immune responses. TLRs (1-9) are expressed throughout the whole intestinal tract by a wide variety of cell types, including intestinal epithelial cells and several immune cells. Recently, expression of TLRs has been found in enteric neurons, glia, and smooth muscle cells of the intestinal wall, structures of the intestinal motor apparatus.

The influence of microbiota and TLRs on intestinal motor activity has been confirmed in our mouse model of intestinal dysbiosis induced by oral administration of antibiotics. Mice with microbiota depletion have less Peyer's patches, enlarged ceca, and mild gut inflammation. Antibiotics decrease fecal output, delay the whole gut and colonic transit, and reduce the spontaneous 
contractions and the responses to the muscarinic agonist acetylcholine (ACh) in ileum and colon. Treatment with antibiotics increases the expression of TLR4, TLR5, and TLR9 in ileum and TLR4 and TLR6 in colon, and it reduces the expression of TLR2 and TLR6 in ileum and TLR2 and TLR9 in colon. Activation of TLR4 by lipopolysaccharide (LPS) reverts the reduction of the spontaneous contractions induced by antibiotics in the ileum. Activation of TLR4 by LPS and TLR5 by flagellin reduces the response to ACh in ileum from control mice.

We have also shown that intestinal microbiota may modulate the intestinal motility by affecting neuroendocrine factors such as serotonin (5-HT). 5-HT is synthesized in the enterochromaffin cells of the intestinal mucosa and in the serotoninergic neurons of the enteric nervous system, and acting as a neurotransmitter, seems to be critical in regulating constitutive gastrointestinal motility. The actions of 5-HT on intestinal motility are transduced by $5-\mathrm{HT}_{2}, 5-\mathrm{HT}_{3}, 5-\mathrm{HT}_{4}$ and $5-\mathrm{HT}_{7}$ receptors, which are expressed in enteric nerves, smooth muscle, and interstitial cells of Cajal. Using our models of TLR $2^{-/-}$, TLR $4^{-/-}$and TLR2/4 double knock-out mice, we have demonstrated that TLR2 and TLR4 modulate the spontaneous contractions and the serotonin contractile responses in mouse ileum and colon, by modifying the expression of the serotonin receptors $5-\mathrm{HT}_{2 \mathrm{~A}}, 5-\mathrm{HT}_{2 \mathrm{~B}}, 5-\mathrm{HT}_{2 \mathrm{C}}, 5-\mathrm{HT}_{3}, 5-\mathrm{HT}_{4}$, and $5-\mathrm{HT}_{7}$.

In conclusion, our results confirm the influence of the microbiota on the regulation of motor function and TLRs expression in mouse intestine. Moreover, TLR2 and TLR4 modulate the motor responses to serotonin in mouse ileum and colon, by affecting several serotonin receptors.

Funding by Gobierno de Aragón (B61/2015) and University of Zaragoza (JIUZ-2013-BIO-08).

Intestine, motor function, microbiota, toll like receptors, serotonin

\section{S9-1}

EFFECT OF LIGHTING IN THE ONTOGENY OF CIRCADIAN RHYTHM

\section{Antoni Diez Noguera \\ Facultad de Farmacia, Basrcelona, Spain}

The circadian rhythm in the rat evolves gradually since the moment of birth changing from a pattern with abundant ultradian components to the absolute dominance of the circadian pattern, a fact that takes place around 2 months of age. There are some unique differences in the evolutionary pattern of males and females. On the other hand, in rats, the exposure to constant light causes the disappearance of the circadian rhythm, but in several experiments a few years ago we discovered that exposure to constant light during the lactation supress this behaviour and allow the animals express the circadian rhythm despite being under constant light. This effect lasts the animal's lifetime, so that exposure to constant light during lactation causes the animals are "resistant" to the effect of light throughout the entire life. Through numerous and complex experiments we were able to stablish a temporal window of sensitivity to the light, and it was located between postnatal days 12 and 20, which coincide with critical moments in the development of the nervous system. Numerous experiments have been conducted to try to determine what change occur in this process, but does not yet have a clear answer, although now we know some features that accompany this phenomenon. There is evidence that several changes in the sensitivity to light take place during this process, although this is not sufficient for an explanation. Currently, the hypothesis that epigenetic changes occur, is being confirmed in recent experiments studying more than two generations of animals.

Circadian rhythm, ontogeny, lighting, rat

\section{S9-2}

OCTODON DEGUS AS AN IDEAL ANIMAL MODEL TO UNDERSTAND HUMAN CIRCADIAN SYSTEM FUNCTIONALITY Beatriz Baño Otálora ${ }^{(1)}$, Maria Angeles Bonmatí Carrión ${ }^{(2)}$, Juan
Antonio Madrid Pérez ${ }^{(2)}$, Maria Angeles Rol De Lama ${ }^{(2)}$

${ }^{(1)}$ University of Murcia. Current address: University of Manchester, Murcia. Current address: Manchester, Spain; ${ }^{(2)}$ University of Murcia, Murcia, Spain

The circadian system generates near $24 \mathrm{~h}$ rhythms and orchestrates endogenous rhythmicity in physiology and behaviour, allowing organisms to anticipate and predict daily changes in their environment. To maintain a stable phase-relationship with the external world, the activity of the master circadian clock in the Suprachiasmatic Nuclei (SCN) is reset daily by the light/dark cycle. Although light intensity and timing are important to synchronise $\mathrm{SCN}$ activity, light spectra containing blue-wavelength also play a fundamental role.

The importance of maintaining a synchronised circadian system in the human brain and body is highlighted by recent studies associating circadian rhythm disruptions (chronodisruption, CD) with different pathological states, which are becoming a public health issue. CD can result from modern Western society lifestyles, including rotational shift work and excessive blue light at night, which are steadily forcing a misalignment between our internal temporal organisation and environmental time cues. Therefore, it is important $i$ ) to understand the effects and the mechanisms involved in CD in order to develop useful therapies and treatments; ii) to design lighting strategies that reduce the disruptive effects of nocturnal blue light in humans without compromising visual acuity. These call for appropriate animal models to be used.

Unlike most animal species used in chronobiology, as humans, the Octodon degus is primarily diurnal with photopic vision. This makes the degu a well suited animal model in which to investigate the effects of lights with different spectra on circadian system functionality. Interestingly, the degu shows chronotype heterogeneity (from strictly diurnal to nocturnal), with some individuals having the ability to shift their activity-phase preference from the day to the dark phase. Likewise, a chronotype continuum, ranging from morning (larks) to night-type (owl), also exists in the human population. The degu, therefore, is also an ideal animal model in which to study the complexity of the human circadian system functionality, and how it can be disrupted by environmental perturbations, such as rotating shift work.

Here, we show that substituting blue-light for shorter-wavelengths could be a good strategy to provide illumination at night with 
minimal disruption to the circadian system in diurnal species. We also establish that a shifting light/dark schedule that mimics lighting conditions experienced by humans during shift work is an experimental condition that causes disruption in the degu's circadian system. Melatonin treatment can be used as an effective therapeutic strategy to enhance circadian rhythmicity under these conditions.

Supported by RETICEF (RD12/0043/0011), MINECO (AYA201571542-REDT, SAF2013-49132-C2-1-R SAF2013-4913, including FEDER cofounding to JAM), Fundación Seneca (19701/PD/14) and FPU- AP2009-1051

Chronodisruption, shift work, Octodon degus, diurnal, light at night, circadian

\section{S9-3}

\section{LIGHT AND BIOLOGICAL RHYTHMS: THE ROLE OF THE RETINA}

Pedro Lax

Universidad de Alicante, Alicante, Spain

The melanopsin system consists of a set of retinal ganglion cells intrinsically photosensitive (ipRGCs), due to the expression of the photopigment melanopsin. In mammals, this photosensitive system provides light information to the suprachiasmatic nuclei of the hypothalamus (the master circadian clock) through the retinohypothalamic tract, allowing entrainment of circadian rhythms to the light-dark cycle. Although melanopsinexpressing retinal ganglion cells (mRGCs) do not require synaptic inputs to generate light-induced signals, they receive inputs from bipolar and amacrine cells, making rod and/or cone signals able to modify the animal's intrinsic response to light. Aging and pathological processes are associated with alterations in circadian rhythms. Ocular pathologies and blindness are thereby associated with circadian disorders that depend on the degree to which light perception is impaired. The higher the degree of light perception a subject has, the more likely their circadian system is to be entrained with normally phased circadian rhythms.

Retinitis pigmentosa (RP) is a heterogeneous group of retinal degenerative disorders of a polymorphic hereditary origin that cause a progressive loss of retinal function and represent a major cause of blindness. Approximately $20-25 \%$ of patients with autosomal dominant RP have a mutation in the rhodopsin gene, one of the most common rhodopsin mutations being the $\mathrm{P} 23 \mathrm{H}$. $\mathrm{P} 23 \mathrm{H}$ transgenic albino rats suffer from a progressive rod degeneration initially associated with normal cone function, which is consistent with the clinical findings in $\mathrm{P} 23 \mathrm{H}$ patients. In this context, it has been demonstrated that retinal degeneration in $\mathrm{P} 23 \mathrm{H}$ rats positively correlates with the occurrence of circadian dysfunctions, that melanopsin-containing ganglion cells degenerate in advanced stages of the disease, and that age-related changes in photosensitive melanopsin-expressing retinal ganglion cells correlate with circadian rhythm impairments in sighted and blind rats. These results indicate that circadian disorders in ocular pathologies and blindness are at least partially due to alterations in the number and morphology of mRGCs.
Parkinson's disease (PD) patients often exhibit sleep disorders, such as excessive daytime somnolence and nighttime sleep fragmentation. Moreover, PD patients exhibit alterations in various circadian patterns, such as in melatonin and cortisol secretion, core body temperature, and heart rate variability, among others. Rotenone is a natural compound, widely used as a broad-spectrum insecticide and pesticide, which causes neuropathological and biochemical defects similar to those found in PD patients, including degeneration of dopaminergic neurons in the substantia nigra. It has been demonstrated that long-term rotenone administration to rats induces loss of vision by promoting photoreceptor cell death and impairment of the dopaminergic retinal system. Moreover, it has been determined that rotenone-induced parkinsonism is associated with alterations in circadian rhythms. These results suggest that circadian disorders in rotenone-treated animals are at least partially due to morphological and functional abnormalities in the retina.

All together, these results demonstrate that degeneration of photoreceptors and inner retinal neurons associated to neurodegenerative diseases have degenerative effects on the number and morphology of mRGCs and causes disturbances in the circadian rhythms.

Melanopsin, ganglion cells, circadian rhythms, retinitis pigentosa, rotenone

\section{S9-4}

\section{CHRONODISRUPTION, MELATONIN AND CANCER}

Juan Carlos Mayo

University of Oviedo, Oviedo, Spain

Most organisms have evolutionary adapted to a 24-hour light:dark rhythm. Suprachiasmatic nucleus (SCN) appear as the master circadian regulator and light is the major 'zeitgeber' working as a circadian entrainment. Additionally, other peripheral regulators occur in most of organism cells. Such adaptation to daily rhythms prepare organism to any environmental change. Among multiple circadian-regulated endocrine parameters, nightly rhythm of pineal melatonin secretion is one of the most important, since this indole itself controls other physiological parameters and exert immunomo dulatory, antioxidant or antitumor activity.

There are a number of physiological parameters that are compromised due to light:dark alterations. This circadian alteration is generally termed as 'chronodisruption', affecting basic processes including sleep:awake rhythms, metabolic disorders or even more serious pathologies such as cancer. In October 2007, The International Agency for Research on Cancer, IARC, recognized the circadian disruption caused by shift-work as a probably carcinogenic to humans, mainly based on accumulated epidemiological evidences highlighting a higher cancer incidence among shiftworkers. Pioneering studies from Schernhammer's lab focused on breast and colon cancer, supported by other, almost simultaneously, publishes studies by other groups, have greatly elevated the interest on this field. These studies prompted other groups to work in the same direction and rapidly new data supported previous findings, thus suggesting that chronodisruption seems to have a major impact on tumor progression. Though clock genes are 
likely involved in the tumorigenic role of circadian disruption, some authors have shown a reduction of night melatonin secretion as a key to explain the critical cue

Interestingly, with some exceptions, not many animal studies mimicking shift-work have tried to elucidate the role of clock genes or melatonin in the chronodisruption-induced tumorigenesis. We have recently shown that melatonin treatment is able to significantly slow down the tumor growth in a prostate cancer transgenic murine model (TRAMP). This model faithfully reproduces the major histopathological features of human prostate cancer. Transgenic TRAMP mice usually show a maximum life span of 42-44 weeks, while melatonin treatment delays the tumor onset and the life span in 12 weeks. These data as well as prostate cancer-cell culture models indicate that melatonin exert an oncostatic activity through the regulation of the IGF pathway, mainly the IGFBP-3. Melatonin is a well-known modulator of insulin secretion, therefore participating in glucose regulation. Additionally, we have reported that melatonin can be intracellularly internalized through GLUT transporters. Regarding metabolic pathways, we have shown that melatonin alters metabolomic pathways in androgen dependent cancer cells.

Since night-shift workers seem to show a higher incidence of prostate cancer and display lower secretory levels of melatonin, and considering that our murine prostate cancer model shows a clear inhibition by the indole, we propose melatonin as one of the keys in the higher incidence of chronodisruption-mediated tumor growth, likely through the regulation of glycolytic metabolism. Furthermore, additional studies focused on the interaction of these metabolic clues, melatonin and clock genes should be taken into consideration.

LAN, chronodisruption, melatonin, cancer

S10-1

METABOLOMICS IN THE STUDY OF HOST-GUT MICROBIOTA INTERACTIONS IN CARDIOMETABOLIC DISEASE

\section{Daniel Monleón}

Fundación para la Investigación del Hospital Clínico Universitario de Valencia, Valencia, Spain

The World Health Organization (WHO) announced in 2013 that cardiometabolic disease is the leading cause of death worldwide. They represent an enormous burden on society, through the loss of health and quality of life and the expenditure of health system resources. The clinical management of these diseases is very complex because they can be present for years before becoming clinically apparent. Understanding its pathogenesis has become increasingly important. The development of specific biomarkers for early detection of cardiometabolic disease and for characterizing new subgroups of patients likely to respond to specific therapies seems essential in risk and patient management. The hostintestinal microbiota co-metabolism appears to be involved in the disease progression. Although the modulation of the microbiota has been postulated as a highly effective therapeutic approach, it is not clear whether all patients can benefit from it and which markers can predict the success of such therapies. The metabolic impact of gut microbiota changes through dietary interventions is highly modulated by the immune system response. Myeloid differentiation primary-response gene 88 (MyD88) is the key signaling adaptor for most TLRs and plays a role in obesity and diabetes. The organ specific deletion of MyD88 has a wide range of diet dependent effects. In this talk, we review the current state of the art in the study of host- gut microbiota metabolic interactions in cardiometabolic disease and the role of metabolomics for its investigation and for identifying early disease biomarkers.

\section{S10-2}

A NOVEL DATA-INDEPENDENT MASS SPECTROMETRY APPROACH ALLOWS HIGHLY DETAILED CHARACTERIZATION OF THE MITOCHONDRIAL INTERACTOME AND REVEALS MECHANISMS OF RESPIRATORY SUPERCOMPLEX ASSEMBLY

\section{Enrique Calvo, José Antonio Enríquez, Jesús Vázquez}

Centro Nacional de Investigaciones Cardiovasculares, Madrid, Spain

The fine-tune stoichiometry of oxidative phosphorylation (OxPhos) mitochondrial respiratory chain components is essential to maintain an efficient metabolism and to minimize ROS production. However, the molecular mechanisms by which the protein components are assembled to form complexes and supercomplexes are still not completely understood.

We have developed a proteomics approach that combines Blue-Native separation of mitochondrial complexes and a newly-developed data-independent mass spectrometry scanning method (Blue-DiS) to perform a highly detailed structural mapping of mitochondrial components. Blue-DiS methodology represents an important improvement in sensitivity for shotgun proteomics and allows the construction of complete fragmentation maps and comprehensive monitoring of selected ions with a very high dynamic range. Computational analysis of the information stored in these unbiased maps using novel in-house algorithms allowed us a high-throughput identification of the protein composition of the OxPhos complexes and supercomplexes, giving a molecular insight on how these proteins dynamically gather together to form the mature complexes. We have also uncovered differences in protein composition from selected tissues and cell lines, suggesting that some proteins behave distinctly in a tissue-specific way.

Correlation analysis of Blue-DiS data also allowed to study novel interactions, confirming several partners that have been recently discovered, as well as a novel candidate to interact with complex I.

Application of Blue-DiS to the study of rat models lacking the protein SCAFI allowed us to discover a novel SCAFI-mediated mechanism of supercomplex assembly.

Finally, preliminary analysis of specific PTMs revealed that these modifications are not evenly distributed across tissues and depend on the size and nature of the supercomplexes, suggesting a post-translational regulation of complex and supercomplex formation.

Mitochondria, mass spectrometry, respiratory complexes 
$\mathrm{S} 10-3$

TRANSCRIPTOMIC ANALYSES TO EXPLORE MECHANISMS OF NUTRIENT ACTION

Jesús De La Osada García

Universidad de Zaragoza, Zaragoza, Spain

Diet influence on atherosclerosis has been well proved in the last century although the defined mechanisms of this association are far away to be understood. In one hand, the nutrient complexity of diets and on the other, the different response in function of a specific genetic makeup make this field an open arena with enormous possibilities. In an attempt to better understand the interactions between nutrients and gene expression, high throughput gene expression using DNA chips has been introduced and applied to analyze transcriptomes. Engineered mouse models of accelerated development of atherosclerosis are providing the ideal battle field where to prove diets of varying nutrient composition and assess the global transcriptional changes in all tissues. With this approach, our lab and others have found a dietary dependent modulation of proteins involved stress-response, cell adhesion, calcium signaling, immune-modulation or metabolic processes not previously related to atherosclerosis. These studies open new opportunities to unveil how diets enriched in a specific component modulate the atherosclerotic process, to realize how dynamic the atherogenic process is, and to understand that the presence of minor components in diets may dramatically change their biological effects. Using this approach, we have explored fatty liver development by using trans-10, cis-12-conjugated linoleic acid (CLA)-enriched diets to promote it and cis-9, trans-11-CLA to ameliorate this effect, suggesting involvement of multiple genes. As an initial screening of candidate genes, only twelve with remarkably modified expression between both CLA isomers were considered and confirmed. Ten genes (Fsp27, Aqp4, Cd36, Ly6d, Scd1, Hsd3b5, Syt1, Cyp7b1 and Tff3) showed significant associations among their expressions and the degree of hepatic steatosis. In hyperhomocysteinemic mice lacking Cbs gene, only Fsp27, $C d 36, S c d 1, S y t 1$ and $H s d 3 b 5$ hepatic expressions were associated with steatosis. In Apoe-deficient mice consuming olive- enriched diet displaying reduction of the fatty liver, only Fsp 27 and Syt 1 expressions were found associated. Expression of these genes is highly associated with hepatic steatosis in a genetic disease such as Cbs deficiency and in two situations such as Western diets containing CLA isomers or a Mediterranean-type diet. Thereby, new processes are involved in lipid handling in liver, and they will help to understand the complex human pathology providing new proteins and new strategies to cope with hepatic steatosis.

Transcriptomics, gene expression, nutrients, microarrays, RNAseq

S10-4

TARGETING MICRORNA FUNCTION THROUGH ENVIRONMENTAL AND LIFESTYLE FACTORS

Alberto Davalos

IMDEA Food Institute, Madrid, Spain
Environmental and lifestyle factors are key players in human health and disease. Dietary factors represent major environmental determinants of human health not only throughout life, but also before conception. Physical activity constitutes another important lifestyle factor with clear effects on health. However, all the mechanisms that underlines the biological effects of these environmental factors remains not fully characterized. Potential mechanisms could involve the interaction of environmental factors with our genome, which is a process largely controlled by epigenetic mechanisms. In fact, several lines of evidence support that environmental factors such as diet and physical activity influence epigenetic mechanisms including small RNA expression, DNA methylation and histone modification. However, the mechanisms by which diet remodel the human epigenome related to small RNA function is poorly understood.

microRNAs (miRNAs) are small noncoding RNAs that post transcriptionally regulate gene expression. By targeting multiple genes, sometimes functionally related, they can control entire biological pathway that control tissue homeostasis. This unique characteristic offers a new therapeutic opportunity to treat a variety of disorders by modulating miRNA function. miRNAs can be released to systemic circulation and are found in almost all biological fluids. Indeed, circulating miRNAs (c-miRNAs) have been shown to participate in cell-to-cell communication and are valuable biomarkers for a wide range of diseases.

Emerging evidence suggest that our diet, through their minor dietary components (i.e. micronutrients), can modulate the expression of miRNAs. Thus, through this mechanism, some dietary components could explain their beneficial effects. In this presentation, we will discuss the novel therapeutic opportunities arising from miRNA modulation by dietary components. We will present specific examples of how certain dietary polyphenols or polyunsaturated fatty acids could modulate the expression of miRNAs and thus regulating different aspects of cellular metabolism. We will also show how nutrition, during critical time of development, could influence offspring health status through miRNAs. Targeting miRNA levels by specific dietary components or lifestyle factors might be a viable option to accompany current pharmacological therapy to target miRNA function.

MicroRNA, Diet, polyphenols, offspring

\section{S11-1}

HYPOTHALAMIC CONTROL OF ENERGY BALANCE IN INSULIN RESISTANCE OBESE MODELS

\section{Cristina Contreras, Miguel López}

CIMUs P0L2 Univ Santiago de Compostela-IDIS, Santiago de Compostela, Spain

Obesity and its associated pathologies, such as diabetes, cardiovascular disease, or some cancer types, have an increasing prevalence, being considered a disease by the HWO. Obesity is caused by a positive energy imbalance in which energy intake is higher energy expenditure. This situation induces the physiological lipid storage in the adipose tissue, however fat can be pathologically 
stored in other organs and tissues, where induces damage, process known as lipotoxicity.

To central level, the energy balance is mainly controlled in the hypothalamus which is composed of several interconnected populations of neurons called nuclei, which receive and integrate a variety of peripheral signals informing to the body energy status to give an afferent response, overexpressing orexigenic peptides as AgrP, NPY, MCH u OXs, which stimulates the food intake, or anorexigenic peptides as POMC y CART that inhibit the food intake. Furthermore, the hypothalamus controls the glucose and lipid metabolism in the peripheral tissues such as liver, pancreas, muscle, white adipose tissue and brown adipose tissue through the autonomic nervous system, either sympathetic or parasympathetic nervous system.

Regarding the brown adipose tissue (BAT), it is mainly present in hibernating mammals and human newborns. However several years ago it was demonstrated its presence in human adults. BAT is characterized by multi-locular lipid droplets and wide presence of mitochondria, produces heat through non-shivering thermogenesis (NST), On the other hand white adipose tissue (WAT) is responsible of energy storage for which accumulates large lipid droplets. BAT and WAT have opposite functional and anatomic properties. The mitochondria use the surrounding fat as fuel to dissipate heat through uncoupling protein 1 (UCP1), process known as thermogenesis.

In the last years, the brown fat and the thermogenesis activation since the hypothalamus have been investigated as possible therapeutic target against obesity and its associated metabolic alterations as insulin resistance.

Obesity, Insulin resistance, Hypothalamus, Brown adipose tissue

\section{S11-2}

\section{ENDOTHELIAL DYSFUNCTION AND VASCULAR AND METABOLIC COMPLICATIONS OF OBESITY}

\section{Dolores Prieto}

Facultad de Farmacia, Universidad Complutense de Madrid, 28040 Madrid, Spain

Obesity is a public health problem of increasing prevalence worldwide and a major risk factor for the development of metabolic and cardiovascular disease including insulin resistance (IR), type II diabetes and hypertension. Obesity is characterized by an excess fat accumulation affecting various cell types including adipocytes, hepatocytes, and immune cells but also vascular smooth muscle (VSM) and endothelial cells. Among adverse cellular reactions to the excess of nutrients (oxidation of non-esterified free fatty acids -FFA- and glucose in the mitochondria and others), increased production of reactive oxygen species (ROS) or oxidative stress occurs and leads to an inflammatory activation of adipose tissue. Hypertrophied adipocytes acquire a pro-inflammatory phenotype and secrete higher amounts of cytokines, adipokines and FFA promoting a low-grade inflammation that causes IR not only in skeletal muscle cells and hepatocytes but also in endothelial cells. The latter promotes endothelial dysfunction and predisposes to both vascular and metabolic dysfunction. Obesity and overweight are associated with impaired endothelium-dependent vasodilatation, a hallmark of endothelial dysfunction early reported in clinical studies showing blunted vasodilator responses correlated with body mass index (BMI) and augmented volume of visceral adipose tissue. Obesity is linked to endothelial dysfunction through indirect mechanisms such as IR and association to risk factors like diabetes mellitus, dyslipidemia and hypertension, or through direct mechanisms including unbalanced production of adipokines and pro-inflammatory cytokines and elevated levels of FFA. Metabolic and flow- or agonist-induced vasodilatation are reduced in obese individuals and experimental models of genetic and diet-induced obesity, compromised bioavailability and abnormal signaling of endothelium-derived nitric oxide (NO) being key pathogenic factors in obesity-associated endothelial dysfunction. Resistance to endothelial NO synthase (eNOS) phosphorylation and NO production in response to flow, growth factors or $\beta$-adrenoceptors underlie impaired NO-mediated arterial relaxations in obese animals. Obesity also blunts prostacyclin-mediated dilatations and augments both COX1- and COX2-dependent vasoconstriction. However, VSM relaxation induced by endothelium-derived hyperpolarization (EDH), which accounts for a major part of vasodilator responses to flow and agonists in small arteries, is preserved or even augmented in obesity and has been suggested to compensate for the reduced $\mathrm{NO}$ and $\mathrm{PGI}_{2}$ bioavailability. Increased plasma levels of endothelin-1 (ET-1) and of endogenous ET-mediated vasoconstrictor activity correlated with BMI have been reported in obese and metabolic syndrome individuals, and obesity enhances expression of arterial $\mathrm{ET}_{\mathrm{A}}$ and $\mathrm{ET}_{\mathrm{B}}$ receptors linked to oxidative stress in both endothelial and VSM cells. Inflammation and oxidative stress are key pathogenic factors in the development of obesity-associated endothelial dysfunction. Inflamed adipose tissue is responsible for abnormal profile of adipokines secretion and elevated release of pro-inflammatory cytokines. Sources of enhanced vascular ROS production in obesity include mitochondria, NADPH and xanthine oxidase and uncoupled eNOS that contribute to superoxide generation and inactivation of NO by forming peroxynitrite. Obesity is one of the main causes of IR that develops in the vasculature well before this response is detected in liver, muscle or adipose tissue. Blunted sensitivity to the NO-mediated vasodilator actions of insulin contributes to the phenotype of the insulin resistant states and its termed "vascular IR". The pathogenic factors responsible for endothelial dysfunction in obesity also deteriorate insulin signaling pathways in endothelial cells, blunt vasodilatation and impair hormone-dependent capillary recruitment and substrate delivery to target tissues, thus contributing to metabolic IR and predisposing to type II diabetes.

Supported by Grant SAF2012-31631 from MINECO (Spain)

Endothelial dysfunction, obesity, oxidative stress

\section{S11-3}

ROLE OF PERIVASCULAR ADIPOSE TISSUE IN OBESITYRELATED VASCULAR DAMAGE

Marisol Fernandez Alfonso

Facultad de Farmacia UCM, Madrid, Spain 
Most blood vessels are surrounded by adipose tissue. Similarly to the adventitia, perivascular adipose tissue (PVAT) was considered only as a passive structural support for the vasculature and it was routinely removed for isolated blood vessel studies. In 1991 Soltis and Cassis demonstrated for the first time that PVAT reduced contractions to noradrenaline in rat aorta. Since then, an important number of adipocyte-derived factors with paracrine vasoactive effects have been identified. Under physiological conditions PVAT releases a number of adipokines that elicit a beneficial effect on vascular function and are essential for the maintenance of vascular resistance. Moderate increases of PVAT in overweight situations lead to an adaptative overproduction of vasodilator factors in PVAT, probably aimed at protecting vascular function. Consequently, in situations of normal weight and moderate overweight the protective and beneficial role of perivascular adipokines increases in parallel to the amount of PVAT. In obesity, however, PVAT undergoes structural and functional changes losing its anticontractile properties by an increase of contractile, oxidative, and inflammatory factors, thus leading to endothelial dysfuntion. This unbalance towards a predominance of vasoconstrictor and inflammatory factors in obesity could provide the link between obesity, cardiovascular functional and structural alterations and cardiovascular diseases. PVAT has been regarded until now as a homogeneous adipose tissue depot which is different from subcutaneous and other visceral adipose tissues. However, a large body of evidence now suggests regional phenotypic differences among PVAT depending on the specific vascular bed or even within different regions of a same blood vessel. PVAT heterogeneity seems to determine its physiological and pathophysiological relevance to vascular function and structure. The adipose-vascular axis may represent therefore novel targets for pharmacological intervention of vasculopathy in cardio-metabolic disorder. (Funded by BFU2011-25303, GR921645-Santander, Fundación Eugenio Rodriguez Pascual, Fundación Mutua Madrileña, and SESCAMET)

Obesity, vascular disease, perivascular adipose tissue, overweight

\section{S11-4 \\ NOVEL GATING MODULATORS OF KCA3.1 CHANNELS AND THERAPEUTIC PERSPECTIVES}

\section{Ralf Köhler}

Aragonese Agency for Research and Development, IIS Aragon, IACS, Zaragoza, Spain

Intermediate-conductance $\mathrm{KCa} 3.1$ channels are expressed in epithelia and endothelia, red and white blood cell lineages, many proliferating cells and cancer cells. In these tissues, KCa3.1 channels control fluid secretion, calcium homeostasis and immune cell function, and mitogenesis by providing membrane hyperpolarization and K-efflux. Therefore the channels are pursued as a potential target for epithelial disorders and edema, immunosuppression, atherosclerosis, fibro-proliferative disorders, and cancer. Concerning the physiological and pathophysiological roles we here review insights that derived from genetic models as well as from patients' material. In addition, explore pathologically relevant interactions with TRPV4 channels in lung tissue. Moreover, we report on recent developments in discovering new $\mathrm{KCa} 3.1$ - gating modulators and critically discuss their therapeutic utilities as well as cardiovascular and neurological safety issues.

Potassium channels, channel gating, ion channel

\section{S12-1}

THE OBSERVATORY OF PAIN. A RESEARCH GROUP FOR THE STUDY OF PAIN IN OUR SOCIAL ENVIRONMENT

Alejandro Salazar, Inmaculada Failde, María Dueñas, Helena De Sola, Begoña Ojeda

Facultad de Enfermería y Fisioterapia, Cádiz, Spain

Pain is a medical and social problem. Indeed, several studies have related the presence of chronic pain (CP) with the appearance of different situations and social problems that form a circle that continuously feeds back. Some of the dimensions of this process are beyond the diagnosis in clinical studies of any kind, making it difficult or even diverting the interpretation of the problem of pain to the purely medical pole, which, despite being the most important, is not the only one. Indeed, there are other factors to be determined which add suffering to patients and their environment, and should be known to be controlled in both medical and socialhealth actions. These other factors are detectable by instruments involving a greater population extension. In this regard, the "Observatories" have been established as valid tools to detect important variables, while creating a body of opinion.

In order to understand, interpret and, if appropriate, make proposals for socio-health intervention, we have launched the Observatory of Pain (OP) under the External Chair of Pain, created by a non-profit agreement between the University of Cadiz and the Grünenthal Foundation. The OP is under cover of a recent agreement of the same characteristics with the Spanish Pain Foundation. The idea of creating this OP came from another activity of the Chair of Pain: the patients' classroom, which has allowed us to maintain close contact with patients suffering from $\mathrm{CP}$ and their families for 3 years, beyond the traditional healthcare environment which represents a medical consultation. The greater availability of time to exchange feelings and perceptions about pain between patients, physicians and relatives, brought out some problems that are rarely addressed in the consultation, and that certainly reinforce the biopsychosocial model of pain. This circumstance, along with the lack of data in our country about the pain from a social approach, led us to conceive the idea of creating an "Observatory" which, like other observatories in different social and health areas, both national and international, allowed to have an updated and real knowledge of the situation of pain in Spain from a global perspective of this problem.

The OP, conceived from this basic principle, aims to act as an organ of analysis and interpretation of available information on pain, to develop a specific information system about the pain that contributes to improve the knowledge of this problem. Its functions include disseminating information to professional collectives and making the knowledge about the situation of pain in our environment available to all stakeholders.

In this context, we have carried out several projects including national population studies, in both general and specific populations, and a validation of a scale. Our first contribution was a study 
to determine the prevalence of pain and its characteristics, and have a view of its impact on the patient, his family, and the health system. We found a prevalence of CP of $16.6 \%$ in Spanish adult population, pain that produces important limitations in patient's daily activities and that affects their emotional and working lives. Parallel to this, we validated the scale of mild cognitive impairment "Test Your Memory" in patients with CP. In our next project, we focused on the perspective of physicians from different specialties in the treatment of pain, showing that the academic load in pain is very limited in the university period, leading to gaps in the knowledge of pain and lack of adherence to guidelines of pain by professionals. With our latest project, called "ACREOPI Study", we aim to know the beliefs, opinions and attitudes of the general population towards the use of opioids in treating pain.

Observatory, Chronic Pain, Validation, Professionals of pain, Opiophobia.

\section{S12-2}

\section{ANIMAL MODELS OF NEUROPATHIC PAIN}

Gema Vera

Universidad Rey Juan Carlos, Alcorcón, Spain

\section{ANIMAL MODELS OF NEUROPATHIC PAIN}

Neuropathic pain has been described as 'the most terrible of all tortures, which a nerve wound may inflict'. It is characterized by sensory abnormalities such as dysesthesia (unpleasant abnormal sensation), hyperalgesia (an increased response to painful stimuli), and allodynia (pain in response to a non-noxious stimulus).

Commonly used painkillers, such as NSAIDS and opiates, have a limited success for treatment of neuropathic pain. Consequently, it is necessary to explore novel therapeutic modalities. Also, the mechanisms involved in its pathophysiology are not entirely understood. Animal models may contribute to the knowledge of neuropathic pain pathophysiology, as well as to evaluate the analgesic potential of novel pharmacotherapies for treating neuropathic pain.

The concept of neuropathic pain includes many clinical situations. Lesions in the central or peripheral nervous system caused by mechanical trauma, metabolic diseases (diabetes), chemotherapeutic drugs, neurotoxic drugs (ethanol), infection (i.e., herpes and HIV) or tumour invasion.

It is very difficult to mimic in an animal model all symptoms and aetiologies of neuropathic pain. The ideal models should result in reproducible sensory deficits such as allodynia, hyperalgesia and spontaneous pain over a sustained period.

Despite these limitations, animal models may help to improve the knowledge of neuropathic pain and provide new therapeutic tools that could be successively translated to effective pain management in the clinical setup.

Animal, neuropathic, pain
S12-3

DRUGS AND OTHER STRATEGIES FOR MANAGEMENT OF NEUROPATHIC PAIN. WHERE ARE WE NOW? WHERE ARE WE GOING?

\section{Raquel Abalo}

Universidad Rey Juan Carlos, Alcorcón, Spain

Neuropathic pain (NP) is one of the most severe types of chronic pain. The International Association for the Study of Pain defines $\mathrm{NP}$ as "pain caused by a lesion or disease of the somatosensory nervous system". It may be associated to different conditions with different anatomical manifestations. Thus, NP may occur symmetrically in diabetes or cancer, due to medications (antiretroviral, chemotherapeutic drugs) or alcohol abuse, to genetic (CharcotMarie-Tooth), autoimmune (amyloidosis), or infectious (postherpetic, HIV) diseases, to vitamin deficiency $\left(\mathrm{B}_{12}\right)$; or may be localized to particular anatomical areas due to trauma or nerve entrapment, radiculopathy, on a limb or facial structures (trigeminal neuralgia, ocular NP)...

Whenever possible, the first measure is to try to remove or control the cause of the problem, once this has occurred, or, ideally, to try to prevent the damage that is expected. However, damage to the nerves may be unavoidable. Neuroinflammation and neural plasticity (peripheral and central sensitization) seem to play a pivotal role in the pathogenesis of NP, leading to increased excitability of sensory neurons and enhanced pain sensitivity.

The complexity of NP explains why clinical treatment options are still limited and unsatisfactory, thus debilitating millions of people worldly and imposing a heavy burden on society. Currently, several treatment modalities exist for the management of NP, including physical therapy, pharmacologic therapy, behavioural medicine, neuromodulation, minimally invasive interventions, and surgery.

Different drugs are presently employed for the management of NP, including anti-depressants, anti-convulsants, topical and local anaesthetics. However, the existing pharmacotherapy has marginal efficacy and significant side effects. Therefore, other agents have been suggested to have potential to attenuate NP in experimental models. These include NSAIDs, corticosteroids, ion channel blockers $\left(\mathrm{Ca}^{2+}, \mathrm{Na}^{+}, \mathrm{K}^{+}\right.$, and TRP channel); ion exchange modulators (NCE and $\mathrm{NHE}$ ); ion/molecule transport modulators (NKCC-1 and glycine); receptor modulators (kinin, histamine, 5$\mathrm{HT}_{1 \mathrm{~A}}$, dopamine, cannabinoid, alpha and beta adrenergic, purinergic, excitatory amino acid, sigma, ORL1, endothelin, melanocortin, ephrin, PAR); enzyme inhibitors (cytosolic kinase, metalloproteinase, protease, vasopeptidase, D-amino acid oxidase, fatty acid amide hydrolase, aldose reductase and sorbitol dehydrogenase) or activators (mTOR); other ligands (modified opioids, AGE, RAGEs, neuropeptides, neurotrophic factors, complement cascade, cytokine, glial cell \& gap junction, nitrous oxide, growth factors, cell adhesion molecules and neuronal sprouting molecules).

With many medications available, it is very common for patients to use multiple agents to try to achieve reasonable pain control. The 
occurrence of adverse effects is indeed very frequent and may contribute to changes in therapy.

Pluridisciplinary approaches may be more effective than drug-only strategies. Thus, management of NP may benefit from the addition of interventional nerve blocks, physical therapy methods (acupuncture, different kinds of electrical nerve stimulation...), psychological aids (learning coping skills), or even optimal nutritional advice, including nutraceuticals and functional foods. Some advanced therapeutic approaches such as neuronal cell transplantation, stem cell therapy, anti-sense oligonucleotide and recombinant therapy have also been evaluated.

Existing therapies for peripheral NP mask the symptoms but they do not affect the underlying problem of nerve loss and dysfunction. At present there is no therapy that can cure or reverse the damage to nerves in peripheral neuropathy in humans. However, as shown in basic research, regeneration can occur provided that there is removal of the underlying trigger, although endogenous regeneration may be incomplete or misdirected.

Finally, some preventive strategies have been tested in models of chemotherapy-induced peripheral pain and NP of traumatic origin. The success of some of these strategies in animal models suggests that prevention of NP development may be achievable, although little clinical evidence supports this at the moment.

We will try to summarize the most promising findings obtained recently with the different strategies mentioned here.

Neuropathic pain, treatment strategies, cannabinoid

\section{ORAL COMMUNICATIONS}

O1-01

ESTROGEN DETRIMENT DURING MENOPAUSE ALTERS ESTROGEN RECEPTOR SIGNALING PLATFORMS LOCATED IN LIPID RAFTS OF CORTICAL BRAIN AREAS, A PHENOMENON INDUCING TOXIC SIGNALLING AND ENSUING ALZHEIMER'S DISEASE

Raquel Marín (1), Ana Canerina-Amaro (1), David QuintoAlemany ${ }^{(2)}$, Fátima Mesa Herrera ${ }^{(2)}$, Carla Ferri ${ }^{(2)}$, José Antonio Pérez ${ }^{(3)}$, Verónica Casañas Sánchez ${ }^{(3)}$, Mario Diaz ${ }^{(2)}$

(1) Laboratory of Cellular Neurobiology, Department of Basic Medical Sciences, Universidad de La Laguna. Associate Research Unit ULL- CSIC "Membrane Physiology and Biophysics in Neurodegenerative and Cancer Diseases"., La Laguna, Spain; ${ }^{(2)}$ Laboratory of Membrane Physiology and Biophysics, Department of Animal Biology, Universidad de La Laguna. Associate Research Unit ULL- CSIC "Membrane Physiology and Biophysics in Neurodegenerative and Cancer Diseases"., La Laguna, Spain; ${ }^{(3)}$ Department of Genetics, University Institute of Tropical Diseases and Public Health, Universidad de La Laguna., La Laguna, Spain.

Estrogens trigger rapid membrane intracellular responses to protect neurons against Alzheimer's disease (AD) pathological events. Consequently, fall in estrogen levels occurring during menopause increases $\mathrm{AD}$ incidence. At the neuronal membrane, estrogens rapidly interact with estrogen receptors (mER) found within lipid rafts. Lipid rafts are microstructures with a particular lipid composition, thus allowing numerous signaling proteins to interact and initiate signal transduction. In these microdomains, mERs appear to aggregate in protein clusters, or signalosomes, anchored to raft scaffolding caveolin-1, complexed with other proteins that participate in neuroprotective responses. Our data have demonstrated that mERsignalosome is disturbed during $\mathrm{AD}$, likely by alterations in the lipid composition of these microstructures. In particular in brain cortex, mER-signalosomes are affected during menopause, observing $\mathrm{mER}$ dissociation of a voltage-dependent anion channel (VDAC1) involved in extrinsic apoptosis and $\mathrm{AD}$ progression, caveolin-1 and other signaling receptors involved in neuroprotection, such as insulin-like growth factor 1 receptor beta. Moreover, mER-VDAC1 dissociation induces channel dephosphorylation, making it more prone to gating, thus enhancing neurotoxicity. Indeed, signalosome molecular disarrangements and VDAC1 dephosphorylation are exacerbated in $\mathrm{AD}$ cortex. Interestingly, lipid rafts from $\mathrm{AD}$ brains exhibit alterations in, both, lipid classes and fatty acids, increasing viscosity and liquid-ordered than in agematched controls. Overall, these data suggest that menopause disrupts lipid raft structure, altering the functionality of $\mathrm{mER}$ and other neuroprotectors integrated in these microdomains, in line with neuropathological events during $\mathrm{AD}$ development.

Supported by SAF2014-52582-R and SAF2014-6144-EXP. A.C.A. holds a fellowship from ACIISI.

\section{References from the Research Group}

1. Marin R. Signalosomes in the brain: relevance in the development of certain neuropathologies such as Alzheimer's disease. Front Physiol. 2011; 2:23-33.

2. Marin R, et al. Estrogen receptors in lipid raft signalling complexes for neuroprotection. Front Biosci (Elite Ed). 2012;4:1420-33.

3. Marin R, et al. Lipid raft disarrangement as a result of neuropathological progresses: a novel strategy for early diagnosis? Neuroscience. 2013;245:26-39.

4. Marin R, et al. Oestrogens as modulators of neuronal signalosomes and brain lipid homeostasis related to protection against neurodegeneration. J Neuroendocrinol. 2013;25(11):1104-15.

5. Marin R. Front Physiol. 2013;4:188.

6. Fernandez-Echevarria C, et al. Neuroscience. 2014;278:354-66.

7. Díaz M, et al. Hippocampal Lipid Homeostasis in APP/PS1 Mice is Modulated by a Complex Interplay Between Dietary DHA and Estrogens: Relevance for Alzheimer's Disease. J Alzheimers Dis. 2015;49(2):459-81.

8. Marin R, et al. Lipid Raft Alterations in Aged-Associated Neuropathologies. Curr Alzheimer Res. 2016 Mar 14.

Estrogens, lipid rafts, Alzheimer's disease, menopause

O1-02

ALTERED SYSTEMIC INFLAMMATORY AND STRESS RESPONSES IN AGED PATIENTS WITH OSTEOARTHRITIS. IMMUNONEUROENDOCRINE DYSREGULATION

Isabel Gálvez ${ }^{(1)}$, María Dolores Hinchado ${ }^{(1)}$, Jorge Guerrero ${ }^{(2)}$, Silvia Torres-Piles ${ }^{(3)}$, Eduardo Ortega ${ }^{(1)}$ 
(1) Departamento de Fisiología (Grupo Inmunofisiología). Facultad de Ciencias. Universidad de Extremadura, Badajoz, Spain; ${ }^{(2)}$ Departamento de Enfermería. Facultad de Medicina. Universidad de Extremadura, Badajoz, Spain; ${ }^{(3)}$ Dpto Terapéutica Médico-Quirúrgica (Grupo Inmunofisiología). Facultad de Medicina. Universidad de Extremadura, Badajoz, Spain

Introduction: Although osteoarthritis (OA) has predominantly been considered a non-inflammatory degenerative arthropathy leading to loss of cartilage, a growing number of evidences consistently suggest that several inflammatory and immunological processes might mediate in the initiation, progression and burden of the disease. In OA, inflammatory mediators are released from various sources and have a deleterious effect on cartilage, bone and synovium. The purpose of the present investigation was to study if this inflammatory response is also reflected in an alteration of the systemic inflammatory and stress responses.

Methods: Eleven patients diagnosed with primary OA according to the American College of Rheumatology (ACR) criteria and eight age-matched healthy volunteers (control group) were enrolled in the study. Serum inflammatory cytokine levels (IL-1 $\beta$, TNF- $\alpha$, IL-8, IL-6, IL-10 and TGF- $\beta$ ) were evaluated by the BioPlex Luminex system. Circulating cortisol levels, as a "neuroendocrine-stress" biomarker, was measured by ELISA.

Results: OA patients showed an inflammatory state accompanied by an altered stress response. This was manifested by high circulating levels of the pro-inflammatory cytokines IL- $1 \beta$, TNF- $\alpha$ and IL8 ; the anti-inflammatory cytokine TGF- $\beta$ and the regulatory cytokine IL-6. There were also decreased systemic levels of cortisol.

Conclusion: An immunoneuroendocrine dysregulation affecting systemic inflammatory and stress mediators underlies OA. This reflects an altered feedback between the inflammatory and stress responses in this pathology.

This investigation has been partially supported by Gobierno de Extremadura-FEDER (GR 15041) and an agreement UEX-“Balneario el Raposo" (036/15).

Osteoarthritis, Immunology, Stress, Inflammation, Cytokines, Cortisol.

\section{O1-03}

\section{CHARACTERIZATION OF VASCULAR SMOOTH MUSCLE CELL PHOSPHATE TRANSPORT}

Luis Hortells, Cecilia Sosa, Víctor Sorribas

Universidad de Zaragoza, Zaragoza, Spain

Hyperphosphatemia plays an important role in the progression of medial aortic calcification. Vascular smooth muscle cells (VSMC) are the most common cell type of the media, and the involvement of increased Pi uptake in the pathogenesis of calcification with hyperphosphatemia was suggested using cultures of VSMC as an in vitro model of the disease.

To understand the role of Pi transport in vascular calcification, the uptake of ${ }^{32} \mathrm{Pi}$ in VSMC has been assayed in different conditions. Total Pi uptake was kinetically characterized, confirming previous michaelian results: $\mathrm{K}_{\mathrm{m}}=0.15 \mathrm{mM} \mathrm{Pi} ; \mathrm{V}_{\max }=440 \mathrm{pmol} / \mathrm{mg}$ prot*min. The pattern of inhibition by several substrates was also studied, including phosphate, bicarbonate, sulfate, arsenate, and phosphonoformic acid. Na-dependent Pi uptake was inhibited by arsenate only, while the Na-independent was inhibited by all of them. In addition, 4,4-diisothiocyanatostilbene-2,2'-disulfonate (DIDS) and 4-acetamido-4'-isothiocyanato-2,2'-stilbenedisulfonate (SITS) partially inhibited both Pi transport components. The effect of $\mathrm{pH}$ in the range 6.0-8.5 was representative of type III Pi transport in the presence of sodium, ie. it decreased with increasing $\mathrm{pH}$, while in the absence of sodium the $\mathrm{pH}$ did not affect Pi uptake. In absence of $\mathrm{Cl}^{-},{ }^{32} \mathrm{P}$ accumulation was higher than in control conditions, suggesting the presence of $\mathrm{a} \mathrm{Cl}^{-}$dependent, Pi efflux system.

In order to identify a Na-independent transport system, several transporters of sulfate and bicarbonate from the families SLC4 and SLC26 were successfully amplified by PCR: SLC4A3 and SLC4A7, as well as SLC26A2, SLC26A6, SLC26A8, SLC26A10 and SLC26A11 (variants 1, 2 and 4). Finally, due to the phenotypic plasticity of the VSMC, real time PCR and Western Blot techniques revealed high expression of Pitl associated with the contractile phenotype, while PiT2 expression was constant. The higher expression of PiT1 was correlated with a higher Pi transport rate in contractile than in proliferative VSMC.

In summary, we have shown that, while the Na-dependent Pi transport in VSMC is due to PiT1 and PiT2 transporters, the Naindependent $\mathrm{Pi}$ uptake is $\mathrm{pH}$-independent, and it is mediated by a transporter with characteristics compatible with the Slc4 and/or Slc26 families of sulfate and bicarbonate transporters. The efflux of Pi from the cells is dependent of chloride in the uptake solution, working most likely in exchange with intracellular Pi.

VSMC, Phosphate transport, Rat.

O1-04

KCNE4 FORMS FUNCTIONAL OLIGOMERIC KV1.3 COMPLEXES IN LEUKOCYTES VIA ASSOCIATION WITH THE CARBOXY TERMINAL DOMAIN OF THE CHANNEL

Sara R Roig ${ }^{(1)}$, Laura Solé ${ }^{(2)}$, Albert Vallejo-Gracia ${ }^{(1)}$, Daniel Sastre ${ }^{(1)}$, Antonio Serrano-Albarrás ${ }^{(1)}$, Ramón Martínez-Mármol (3), Michael M Tamkun ${ }^{(2)}$, Antonio Felipe ${ }^{(1)}$

(1) Molecular Physiology Laboratory, Departament de Bioquímica i Biomedicina Molecular, Institut de Biomedicina (IBUB), Universitat de Barcelona, Barcelona, Spain; ${ }^{(2)}$ Department of Biomedical Sciences, Colorado State University, Fort Collins, Colorado, United States; ${ }^{(3)}$ Clem Jones Centre for Ageing Dementia Research, Queensland Brain Institute, The University of Queensland, Brisbane, Queensland, Australia

The voltage-dependent potassium channel Kv1.3 plays crucial roles in the immune system. We have previously described that KCNE4, present in leukocytes, physically interacts with Kv1.3 acting as a dominant negative subunit. KCNE4 inhibits $\mathrm{K}^{+}$currents and impairs membrane targeting. Although canonical $\mathrm{KCNE}-\mathrm{Kv} 7$ interactions have been under intense investigation, the molecular determinants implicated in other essential KCNE interactions remain unknown. Thus, structural interacting signatures between Kv7 and Kv1 channels display enormous differences. Our results point to the carboxy terminal of Kv1.3 as an essential and sufficient anchoring domain for KCNE4. However, 
similar to what described for the Kv7.1 association, this motif apparently is not implicated in the Kv1.3 gating modulation. In addition, we describe that two independent and synergic mechanisms potentiate the KCNE4-dependent intracellular retention of the Kv1.3/KCNE4 complex. First, KCNE4 association masks the YMVIEE signature at the C-terminal domain of the Kv1.3, which is crucial for the channel surface targeting; and second, we identify a potent basic endoplasmic reticulum retention motif in KCNE4. This signature is transferred to the channelosome that further limits cell surface expression and forces an ER localization. Our results define specific molecular determinants which play a crucial role in the physiological function of Kv1.3 in leukocytes.

Supported by MINECO, Spain (BFU2014-54928-R and BFU201570067-REDC).

\section{O1-05}

MELATONIN REGULATES THE SPHINGOSINE KINASE 1/SPHINGOSINE 1-PHOSPHATE SIGNALING PATHWAY IN A MICE MODEL OF DIETHYLNITROSAMINE-INDUCED HEPATOCELLULAR CARCINOMA

D.I. Sánchez ${ }^{(1)}$, Bárbara González-Fernández ${ }^{(1)}$, Beatriz SanMiguel ${ }^{(1)}$, Irene Crespo ${ }^{(2)}$, Juan Ortíz De Urbina ${ }^{(3)}$, Javier González-Gallego $^{(2)}$, María J Tuñón ${ }^{(2)}$

(1) Institute of Biomedicine (IBIOMED), University of León, León, Spain; ${ }^{(2)}$ Institute of Biomedicine (IBIOMED), University of León; Centro de Investigación Biomédica en Red de Enfermedades Hepáticas y Digestivas (CIBERehd), León, Spain; (3) Pharmacy Service, Complejo Asistencial Universitario de León, León, Spain

Hepatocellular carcinoma (HCC) is the third leading cause of cancer-related deaths globally characterized by high malignancy, aggressive progression, and limited therapeutic options owing to the poor understanding of HCC development mechanism. Metabolites of sphingolipids have emerged as key signaling molecules in cancer progression. Thus, the modulation of sphingolipids metabolism has been shown to have potential as a cancer therapeutic target. Besides, melatonin, a product of the pineal gland, is reported to be able to alleviate promotion and progression on a variety of tumors including HCC. In this regard, we investigated whether melatonin ameliorates HCC progression in mice treated with diethylnitrosamine (DEN) by regulating the sphingosine kinase 1/sphingosine 1-phosphate (SphK1/S1P) axis. Mice in treatment groups received DEN (35 mg/kg body weight i.p) once a week for 8 weeks. Melatonin was given at 5 or $10 \mathrm{mg} / \mathrm{kg} /$ day i.p. beginning 4 weeks after the onset of DEN administration and ending at the sacrifice time per each group (10, 20, 30 and 40 weeks). To determine whether melatonin treatment alleviates the marked upregulation of the hepatic sphingosine pathway in mice with DENinduced HCC, qRT-PCR, western blot, immunohistochemistry, and ELISA test were performed. Our data showed that the SphK1 increased significantly the expression of mRNA, protein concentration, and liver immunostaining in DEN-treated groups. S1P levels, a cancer-related biomolecule catalyzed by SphK1, were obtained from ELISA test that showed values which were significantly higher in DEN-treated mice when compared with control groups and groups receiving melatonin. On the contrary, sphingosine phosphate lyase 1 (SGPL1), the only enzyme that can irreversibly degrade the bioactive sphingolipid S1P, mRNA and protein levels were markedly diminished. We, also, evaluated SphK receptors (S1PRs) owing to their implication in the activation of downstream signaling pathways that modulate cell differentiation, growth and proliferation, inflammation, and apoptotic signals, along with cancer cells invasiveness. As expected, S1PR1 and S1PR3 mRNA levels, and the protein expression, were significantly upregulated in DEN-treated mice from the early onset of the experiment. The mRNA levels and protein expression of S1PR2, a receptor expressed in tumor cells that mediates inhibition of cell migration and invasion, decreased in mice receiving DEN. Noteworthy, melatonin inhibited S1P production, lowered expression of SphK1, S1PR1, S1PR3, and increased the expression of SGPL1 and S1PR2 in a dose-dependent manner. The data obtained suggest that melatonin might protect the liver from DENinduced injury and HCC by regulating SphK1/S1P signaling pathway. Supported by grants from the AECC.

Melatonin, sphingosine kinase 1, sphingosine 1-phosphate, diethylnitrosamine, hepatocellular carcinoma.

01-06

AGING AND SARCOPENIA. ROLES OF THE INNATE IMMUNITY AND MELATONIN THERAPY

Marisol Fernández Ortiz, Ramy K. Sayed, Juan Ángel Rodríguez Pozo, Paula Aranda Martínez, Darío Acuña Castroviejo

Centro de Investigación Biomédica, Parque Tecnológico de Ciencias de la Salud, Avda. del Conocimiento s/n, Granada, Spain

Inflammaging describes the low-grade, chronic, asymptomatic, systemic inflammation in aging, in the absence of overt infection. The large variety of the stimuli fuelling inflammaging apparently converges on few basic mechanisms and pathways based on innate immunity, such as activation of NF-kB and NLRP3 inflammasome, which are responsible for the production of inflammatory molecules. Inflammaging is a highly significant risk factor for both morbidity and mortality in the elderly people and increasing evidences support that this subclinical inflammation during aging is associated with chronic diseases and sarcopenia, which is characterized by loss of strength and muscle mass. On the other hand, extrapineal melatonin is known for its antioxidant activity, its immunomodulating properties and its physiological role on mitochondria. Due to its antiinflammatory and antioxidant properties, we hypothesized that melatonin could be used to improve the symptoms of sarcopenia. To assess the age-related role of innate immunity on the development of sarcopenia and to study the effects of melatonin on this immunosenescent process, 3 and 12 months-old wild type (WT) and NLRP3 deficient $\left(\mathrm{NLRP}^{-/}\right)$mice were studied. The latter were treated with $10 \mathrm{mg} / \mathrm{kg}$ melatonin in the diet for $2 \mathrm{months}$, from 10 to 12 months of age. Locomotor activity (total distance, resting time, mean speed, and maximal speed) was studied using a Smart Video Tracking System, and speed and fatigue were tested with a treadmill system (running distance and time of exhaustion). All experiments were conducted in accordance with the University of Granada's Ethical Committee; the Spanish Protection Guide for Animal Experimentation (R.D. 53/2013), and the European 
Convention for the Protection of Vertebrate Animals used for Experimental and Other Scientific Purposes (CETS \# 123). The results showed that $\mathrm{NLRP}^{-/-}$mice behaved better than WT mice at the same age in terms of resting time, maximal speed, running distance, and time to exhaustion. In both animal models, administration of melatonin significantly improved all parameters of muscle efficiency, even above the performances measured in 3 months old mice. It is concluded that NLRP 3 exerts a type of brake in the motor activity of mice, which may have important consequences when it is activated during inflammaging. Moreover, we also concluded the high efficacy of melatonin to improve skeletal muscle performance. Both findings can have clinical interest in preventing sarcopenia associated to frailty in aged people.

Supported in part by grants no. PI13-00981 and RD12/0043/0005

Aging, sarcopenia, inflammaging, innate immunity, melatonin.

\section{O1-07}

NLRP3 INFLAMMASOME AFFECTS MITOCHONDRIAL MORPHOLOGY IN SKELETAL MUSCLE OF MICE

Ramy K. Sayed, Marisol Fernández Ortiz, Ibtissem Rahim, Darío Acuña Castroviejo

Centro de Investigación Biomédica, Parque Tecnológico de Ciencias de la Salud, Avda. del Conocimiento s/n, Granada, Spain

Pathogens in vertebrates are recognized and eliminated by both innate and adaptive immune systems. Once the former one is activated, it can sense a wide range of pathogens through pattern-recognition receptors (PRRS). Nod-like receptors (NLRs) are a family of PRRs that expressed in cytosol, and recognize intracellular pathogen and danger-associated molecular patterns (PAMPs and DAMPs). Certain NLRs including NLRP3 form large cytoplasmic complexes, termed inflammasomes, which intern activate caspase cascades resulting in proteolytic activation of proinflammatory cytokines. Interestingly, innate immune responses are regulated by mitochondria through reactive oxygen species (ROS)-dependent activation of NLRP3 inflammasomes. To investigate the effect of NLRP3 inflammasome on the morphology and ultrastructure of the gastrocnemius muscle and mitochondria, 3 months-old wild type (WT) and NLRP3 deficient $\left(\mathrm{NLRP}^{-/-}\right)$mice, were studied. All experiments were conducted in accordance with the University of Granada's Ethical Committee; the Spanish Protection Guide for Animal Experimentation (R.D. 53/2013), and the European Convention for the Protection of Vertebrate Animals used for Experimental and Other Scientific Purposes (CETS \# 123). Animals were anaesthetized by intraperitoneal injection ofequithesin, transcardially perfused by trump's fixative, and then the gastrocnemius muscle was dissected and processed for light and transmission electron microscopical (TEM) analysis. Different morphometrical measurements were studied on the acquired digital images. The results showed that gastrocnemius muscle fibers of $\mathrm{NLRP}^{-1-}$ mice had larger cross section area (CSA) than that of the wild type mice at the same age, with the fibers closed to each other and showed less collagenous connective tissue infiltrations in epimysium and endomysium. WT mice revealed few small sized vacuolated mitochondria with damaged cristae, In contrast, $\mathrm{NLRP}^{-/-}$mice revealed a significant increase in the number of intermyofibrillar (IMF), and CSA, perimeter and diameter of both IMF and subsarcolemmal (SS) mitochondria with numerous cristae, while number of SS mitochondria was significantly reduced. No significant changes in the length of sarcomeres and A-, I-, and H-bands were detected. The results here reported indicate that NLRP3 inflammasome may affect negatively mitochondrial morphology, leading to their dysfunction and damage after its activation by inflammatory signals. These changes may reflect on the efficacy of the skeletal muscle and ultimately loss of its strength.

Supported in part by grants no. PI13-00981 and RD12/0043/0005, by grants from the Egyptian Cultural Bureau in Madrid, Egyptian Ministry of Higher Education, Egypt

CSA, Mitochondria, Muscle fibers, NLRP3 inflammasome.

O1-08 STUDY OF ULTRAVIOLET RADIATION EFFECT ON
FIBROBLASTS AND QUERCETIN PROTECTION

Alvaro Casanova Flor De Lis, Desiree Pereboom, Jose Octavio Alda

Universidad de Zaragoza, Zaragoza, Spain

Summary: Most solar adverse effects are due to the action of ultraviolet radiation (UV) on the skin, causing cellular apoptosis and necrosis, skin redness and ocular inflammations. Actual protection against these forms of radiation uses external barriers, such as solar filters or photo blocks, so that achieving effects at a cellular level would multiply this protection without blocking the physiological functions of solar radiation.

Since one of the sources of this cell death originates in free radicals and since quercetin flavonoid is an excellent free radicals scavenger, this research is aimed at quantifying the possible protective effects of flavonoid against the damage produced by ultraviolet radiations.

Methods: The study model is human fibroblast primary cell culture. These model has been used for mechanism of action radiations study and for quercetin treatment and irradiation study.

Oxidative stress and the different cells deaths determinations were performed by image cytometry (ImageStream X, Amnis) with cFDA (Carboxyfluerescein diacetate)/IP(Propidium iodide), HE (Hidroetidine), DHR123(Dihydrorhodamine 123), ADPA (Anthracenedipropionic acid) and Annexin V-IP Kit.

Results: UV DL ${ }_{50}$ (lethal dose $50 \%$ ) was determined of $6.97 \mathrm{~J} /$ $\mathrm{cm}^{2}$ and $1.269 \mathrm{~J} / \mathrm{cm}^{2}$ for UVB and UVC respectively with a significant increase of superoxide anion, hydrogen peroxide and oxygen singlet. The most part of the damage was observed in early apoptosis for UVB and late apoptosis for UVC.

Also the results showed how very low doses of quercetin (1-5 $\mu \mathrm{M})$ proved able to reduce the damage produced by ultraviolet radiation ( $\pm 60 \%$ UVB and $\pm 25 \%$ UVC), decreasing the mortality, and mostly reducing the apoptosis. Damaging species such as superoxide radical, singlet oxygen and peroxide hydrogen were also reduced (until $90 \%$ ). 
Quercetin proved able to reduce the damage of mitochondrial membrane potential.

Conclusion: The results showed how very low doses of quercetin are able to reduce the damage produced by UV radiation, decreasing mortaliy (mostly reducing the apoptosis) and oxidative stress. Quercetin proved able to selectively killed senescent and damaged cells.

Ultraviolet radiation, skin, oxidative stress, apoptosis, quercetin and image cytometry

$\mathrm{O} 2-01$

\section{MUSCLE DAMAGE AND OXIDATIVE STRESS DURING BENCH PRESS AND SQUAT EXERCISES}

Jerónimo Aragón Vela ${ }^{(1)}$, Yahira Barranco Ruiz ${ }^{(2)}$, Cristina Casals Vázquez ${ }^{(1)}$, Jesús F. Rodríguez Huertas ${ }^{(1)}$, Julio Plaza Díaz ${ }^{(1)}$, Luis Fontana Gallego ${ }^{(1)}$, Rafael Casuso Perez ${ }^{(1)}$

(1) Institute of Nutrition and Food Technology "José Mataix", Biomedical Research Centre, Department of Physiology, Faculty of Sport Sciences, University of Granada, Granada, Spain., Granada, Spain; ${ }^{(2)}$ School of Physical Culture, Faculty of Health Sciences, National University of Chimborazo Riobamba, Ecuador, Chimborazo, Ecuador

Background: Resistance exercise, especially when it consists in eccentric contractions, can induce skeletal muscle damage due to high mechanical stimuli and increased oxidative stress. Moreover, the amount of muscle mass engaged during resistance test may impact on the exercise-induced muscle damage and oxidative stress.

Aim: To test skeletal muscle damage and oxidative stress in response to resistance exercises with different amounts of involved muscle mass.

Methods: Ten healthy males experimented in strength training ( $22 \pm 0.77$ years old) performed two maximum incremental strength test (bench press vs squat) both consisted of five incremental intensities: 20, 40, 60, 80 and $100 \%$ of the one repetition maximum. The first three intensities consisted of five repetitions interspersed with $3 \mathrm{~min}$ of recovery, only two repetitions were performed in the $80 \%$ intensity and one repetition in the $100 \%$ with 5 min of recovery between sets. Prior to test, the one repetition maximum was calculated for each participant. Blood samples were taken at rest, 15-min post-exercise, and $24 \mathrm{~h}$ post-exercise. Serum creatine kinase, lactate dehydrogenase, alpha-actin and hydroperoxides were analysed. Statistical comparisons were determined using a mixed two-way analysis of variance followed by Bonferroni post-hoc test. Data are expressed as mean \pm standard error of the mean. The level of significance was set at $p<0.05$.

Results: Serum creatine kinase levels, 15-min after exercise, were significantly higher in bench press $(26.43 \pm 1.94 \mathrm{U} / \mathrm{L})$ than in squat exercise $(19.09 \pm 2.04 \mathrm{U} / \mathrm{L})(p=0.017)$. Accordingly, lactate dehydrogenase values were higher in response to bench press exercise $(368.26 \pm 24.22 \mathrm{U} / \mathrm{L})$ compared with squat exercises (306.27 $\pm 18.48 \mathrm{U} / \mathrm{L})(p=0.057)$. However, alpha-actin values were not shown significant changes between bench press $(107.06 \pm 3.12 \%)$ and squat exercise $(103.76 \pm 3.12 \%)(p=0.497)$. Moreover, lipid peroxidation responses were similar between tests $(p=0.526)$.

Conclusion: At equivalent workloads, the bench press exercise generates greater parameter of muscle damage (CK and $\mathrm{LDH})$ than squat exercise; probably because the latter has a higher muscle recruitment inducing lower muscle-specific workload and, consequently, lower muscle damage. Nevertheless, the parameters of oxidative stress assessed were similar between tests.

Muscle groups, creatine kinase, lactate dehydrogenase, resistance exercise and muscle involvement

$\mathrm{O} 2-02$

SPRINT INTERVAL TRAINING INDUCES A WEAK OXIDATIVE-RELATED TRANSCRIPTIONAL RESPONSE WITHIN THE TRICEPS BRACHII OF TRAINED SWIMMERS

Jesus R Huertas ${ }^{(1)}$, Julio Plaza-Diaz ${ }^{(1)}$, Jerónimo Aragon-Vela ${ }^{(1)}$, Daniel Camiletti-Moiron ${ }^{(2)}$, Francisco J Ruiz-Ojeda ${ }^{(1)}$, Cándido Robles-Sanchez ${ }^{(1)}$, Alberto Ruiz-Ariza ${ }^{(3)}$, Antonio MartínezAmat ${ }^{(4)}$, Marina Hebberecht ${ }^{(5)}$, Carlos Melero-Romero ${ }^{(1)}$, Luis M Salmeron ${ }^{(5)}$, Rafael A Casuso ${ }^{(1)}$

(1) Institute of Nutrition and Food Technology "José Mataix", University of Granada, Armilla (Granada), Spain; ${ }^{(2)}$ Department of Physical Activity, University of Cadiz, Cádiz (Spain), Spain; ${ }^{(3)}$ Department of Physical Activity, University of Jaén, Jaén (Spain), Spain; ${ }^{(4)}$ Department of Health Sciences, University of Jaén, Jaén (Spain), Spain; ${ }^{(5)}$ San Cecilio University Hospital, Granada (Spain), Spain

Sprint interval training (SIT) has been recommended to athletes as a time-efficient training stimuli in order to improve skeletal muscle oxidative and glycolytic metabolism. While SIT has been tested for running and cycling, little is known about the molecular pathways triggered by SIT in swimmers. We aimed to compare the molecular mechanisms induced by SIT and moderate intensity (MOD) swimming in the skeletal muscle of highly trained swimmers.

For that purpose we recruited nine swimmers enrolled in swimming competition for at least 8 years. They had an average age of 23.0 year (range 19-26 years) and a maximal swimming speed of $2.0 \mathrm{~m} / \mathrm{s}$ (range $1.88-2.10 \mathrm{~m} / \mathrm{s}$ ). All the swimmers underwent three experimental days. The first day they attended to the laboratory where a biopsy from the long head of the triceps brachii was taken to stablish the basal transcriptional activity. Then the swimmers performed two swimming session on separate days; MOD consisted in $10 \times 200 \mathrm{~m}$ separated by $40 \mathrm{~s}$ at a constant speed trying to maintain the highest speed average. SIT consisted in $10 \times 50 \mathrm{~m}$ all out swimming every $4 \mathrm{~min}$. A muscle sample was obtained immediately after swimming $(0 \mathrm{~h})$ and after $3 \mathrm{~h}$ of recovery $(3 \mathrm{~h})$. Statistical significance was set at $p<0.05$. The duration of the session was similar between SIT (36 min) and MOD ( $32 \mathrm{~min})$. Blood lactate within SIT was higher at every time-point examined but peaked $7 \mathrm{~min}$ post exercise $(13.8 \pm 0.77)$ while MOD peaked 3 min post exercise $(9.4 \pm 2.5)$. In response to SIT there was a significant increase of the mRNA levels of the master regulator of the mitochondrial biogenesis PGC- $1 \alpha$ at $3 \mathrm{~h}$. In 
addition the AMP-related kinase AMPK and the regulator of cellular growth S6K1 increased in response to SIT at $0 \mathrm{~h}$. MOD induced a greater transcription of PGC- $1 \alpha$ at $3 \mathrm{~h}$ than SIT, and the angiogenic factor VEGF increased at $3 \mathrm{~h}$ in response to MOD. The regulator of ribosome biogenesis c-Myc began its transcription at $0 \mathrm{~h}$ and further increased at $3 \mathrm{~h}$ in response to both swimming protocols.

Our results suggest that SIT and MOD induce a similar activation of the cellular growth pathway, but both protocols differ on the timing of the signal as $\mathrm{S} 6 \mathrm{~K} 1$ is induced at $0 \mathrm{~h}$ post SIT while in response to MOD S6K1 increase at $3 \mathrm{~h}$. The greater transcription of PGC- $1 \alpha$ induced by MOD along with the effect on VEGF suggest that SIT compromise the angiogenic and mitochondrial remodelling response in swimmers.

Swimming, skeletal muscle, mitochondria, angiogenesis

\section{MECHANISMS INVOLVED IN THE ORIGIN OF SKELETAL MUSCLE DAMAGE AFTER A SUBMAXIMAL TEST IN EN- DURANCE PRACTITIONERS}

Yaira Barranco-Ruiz ${ }^{(1)}$, Cristina Casals ${ }^{(2)}$, Jerónimo Aragon-Vela (2), Antonio Martínez-Amat ${ }^{(3)}$, Rafael A. Casuso ${ }^{(2)}$, Jesús R. Huertas ${ }^{(2)}$

(1) Universidad Nacional de Chimborazo, Riobamba, Ecuador; ${ }^{(2)}$ Universidad de Granada, Granada, Spain; ${ }^{(3)}$ Universidad de Jaén, Jaén, Spain

Background: Endurance training may generate metabolic stress with an important impact on skeletal muscle function. Skeletal muscle damage is usually attributed to mechanical stress; however, metabolic stress can also contribute to generate it.

Aim: To determine whether the skeletal muscle damage generated after a submaximal incremental test on endurance practitioners is primarily due to the metabolic or mechanical stress, and what role the volume of endurance practice plays.

Methods: Forty nine healthy males $(37.58 \pm 1.82$ years old) with different volume of endurance practice (hours/week) underwent the submaximal and incremental test PWC 170. Blood samples were collected at rest and after the test. The alpha-actin release in serum was analyzed by Western Blot as a skeletal muscle damage marker of sarcomeric origin. Immunoassays were performed to determine the serum cardiac troponin I concentrations (TnI). Blood lactate concentrations were measured as a metabolic stress marker. Mechanical work performed in the submaximal test was also measured. Multiple linear regression analyses (stepwise method) were conducted to explore the relationships between study variables. The model 1 included the blood lactate concentrations post-effort and the mechanical work performed during the submaximal test as predictors of the release of alpha-actin post-effort. A linear regression with the hours of endurance practice per week as predictor of alphaactin concentration post-effort was also performed. Model 2 incorporated blood lactate concentrations post-effort and mechanical work as predictor of volume of endurance practice.

Results: Serum cardiac troponin I concentrations were in normal values and below the immunoassay detection limits values both baseline and after submaximal test (baseline $=0.078$ $\pm 0.0019 \mathrm{ng} / \mathrm{ml}$; after test $=0.011 \pm 0.0023 \mathrm{ng} / \mathrm{ml})$; thus alphaactin concentrations represented the skeletal muscle damage. The mechanical work significantly predicted the alpha-actin levels post-effort $(\mathrm{R} 2=0.227, p=0.001)$. Non-significant association between the blood lactate post-effort concentration and alphaactin levels post-effort was found. The hours of endurance training predicted the alpha-actin concentrations $(\mathrm{R} 2=0.706, p<0.001)$. The mechanical work significantly predicted the hours of training $(\mathrm{R} 2=0.359, p<0.00)$.

Conclusions: The data reinforce the mechanical stress prevalence over the metabolic stress as a predictor of skeletal muscle damage in submaximal incremental tests on endurance practitioners. In addition, endurance training hours practiced per week are exclusively predicted by the mechanical work developed in submaximal test. Thus, it seems that for the same metabolic stress (same blood lactate concentration post-effort) the endurance practitioners with higher volume generate more alpha-actin than the rest.

Metabolic stress, mechanical stress, blood lactate concentrations, alpha-actin, aerobic training, skeletal muscle damage

\section{O2-04}

PRO-INFLAMMATORY CYTOKINES (IL-6 AND TNF-A) AND THEIR SOLUBLE RECEPTORS IN ELITE JUDO ATHLETES

Cristina Casals ${ }^{(1)}$, Raquel Escobar-Molina ${ }^{(2)}$, Yaira BarrancoRuiz ${ }^{(3)}$, Jeronimo Aragon-Vela ${ }^{(1)}$, Silvia Rosillo ${ }^{(1)}$, Jesús R Huertas ${ }^{\text {(1) }}$

(1) Institute of Nutrition and Food Technology, Biomedical Research Centre, Faculty of Sport Sciences, University of Granada, Granada, Spain; ${ }^{(2)}$ Department of Physical Education and Sports, Faculty of Sport Sciences, University of Granada, Granada, Spain; (3) School of Physical Culture, Faculty of Health Sciences, National University of Chimborazo, Riobamba, Ecuador

Inflammatory biomarkers are widely used in sport research to evaluate the elite athlete and adapt the training program. Nevertheless, some receptors of pro-inflammatory cytokines are not commonly assessed in athletes and might be relevant. Thus, the aim of the study was to describe pro-inflammatory cytokines and their soluble receptors in elite athletes at rest and after an exercise test. Twenty judo athletes from the Spanish National Team (10 males, 10 females) participated in this study during a competitive period $(24.1 \pm 3.2$ years old). All athletes performed a specificjudo test (the Special Judo Fitness Test) consisted in a shortduration, high-intensity intermittent effort. Blood samples were collected from the antecubital vein at rest and 15 -min after the test, the samples were centrifuged and storage at $-80{ }^{\circ} \mathrm{C}$ until further analysis. Plasma interleukin-6 (IL-6) and tumour necrosis factor $(\mathrm{TNF}-\alpha)$ levels, as pro-oxidant cytokines, and their respective soluble receptors (sIL-6R $\alpha$ and sTNF-R1), as anti-inflammatory markers, were determined by Immunology Multiplex Assay (HSTCMAG-28SK and HSCRMAG-32 K, Merck Millipore, Darmstadt, Germany) and the Luminex ${ }^{\circledR} 200^{\mathrm{TM}}$ System (Luminex Corp., Austin, TX, USA) according to the manufacturer's instructions. Normality of distribution and homoscedasticity were checked with Shapiro-Wilk and Levene tests. Statistical 
differences between moments were analysed by the pairedsamples $T$ test for pre-post comparisons. Our results showed similar IL-6 and sIL-R $\alpha$ levels before and 15-min after the effort; however, TNF- $\alpha$ and sTNF-R1 significantly increased in response to exercise $(0.21 \pm 0.33 \mathrm{pg} / \mathrm{mL}, p=0.011 ; 194.87 \pm 274.20 \mathrm{pg} / \mathrm{mL}$, $p=0.005$; respectively). For that, sTNF-R1 can be an interesting marker for assessed inflammatory responses to short duration, high-intensity intermittent efforts in elite judo athletes when TNF- $\alpha$ is determined; although to our best knowledge, there is no study reporting this receptor in athletes. Moreover, the mean values of inflammatory markers were $0.32 \pm 0.12 \mathrm{pg} / \mathrm{mL}$ for IL-6, $10249.36 \pm 6346.47 \mathrm{pg} / \mathrm{mL}$ for sIL-6R $\alpha, 0.94 \pm 0.31 \mathrm{pg} / \mathrm{mL}$ for TNF- $\alpha$, and $524.39 \pm 229.02 \mathrm{pg} / \mathrm{mL}$ for sTNF-R1. Therefore, all judokas presented high plasma concentrations of sIL-6R $\alpha$ and STNF-R1; and the relation IL-6/sIL-6R $\alpha$ was $1: 100,000$ and the TNF- $\alpha /$ sTNF-R1 was $1: 1,000$. These results suggest that elite judo athletes have developed beneficial adaptations to exercise and presented high-performance level during the competitive period despite the demanding training loads and the high-intensity effort.

Inflammation, martial arts, exercise, performance

\section{O2-05}

EFFECTS OF AEROBIC EXERCISE ON THE ELECTROPHYSIOLOGICAL HETEROGENEITY OF THE MYOCARDIUM DURING VENTRICULAR FIBRILLATION IN A MODEL OF ISOLATED RABBIT HEART. AN EXPERIMENTAL STUDY

Javier Simón-Grima ${ }^{(1)}$, Carlos Soler ${ }^{(1)}$, Luis Such-Miquel ${ }^{(1)}$, Irene Del Canto ${ }^{(2)}$, Manuel Zarzoso ${ }^{(1)}$, Óscar Julián Arias-Mutis ${ }^{(1)}$, Patricia Genovés ${ }^{(1)}$, Francisco Javier Chorro ${ }^{(1)}$, Luis Such ${ }^{(1)}$, Antonio Manuel Alberola ${ }^{(1)}$

(1) Universitat de València (E. G.), Valencia, Spain; ${ }^{(2)}$ Fundación de Investigación, Hospital Clínic Universitari de Valencia (INCLIVA), Valencia, Spain

Introduction: Aerobic exercise can modify the electrophysiological properties of the heart by reducing the heterogeneity of the myocardium. It is known the protective effect of exercise against sudden cardiac death, among other circumstances, for its demonstrated effects on cardiac electrophysiology.

Purpose: To investigate the effect of aerobic training ventricular fibrillation (VF) on two cardiac electrophysiological parameters of heterogeneity which are the dominant frequency (DFr) and the spectral concentration $(\mathrm{SpC})$. The study was carried out in a model of isolated rabbit heart immersed in a thermostatic bath as a prelude to global myocardial ischemia normothermic studies.

Methods: 15 New Zealand White male rabbits were divided into two groups (8 controls and 7 trained-on a treadmill-). After heparinization and euthanasia (thiopental $200 \mathrm{mg} / \mathrm{kg}$, according to European ethical guidelines), the hearts were removed and placed in a Langendorff setup. Stimulation electrodes and a recording multielectrode (256 electrodes) were placed on the epicardium of the left ventricle. VF (with nonstop perfusion) was triggered by means of pacing the left ventricle at increasing frequencies. The heart was totally immersed from the beginning in Tyrode into a thermostatic bath at $37.6{ }^{\circ} \mathrm{C}$. VF recordings were made during $5 \mathrm{~min}$ to observe the temporal evolution of the DFr and the $\mathrm{SpC}$. DFr and SpC were analyzed by spectral methods. Significance was reached when $p<0.05$.

Results: DFr was lower in the trained group than the control group $(18.5 \pm 1.5$ vs $23.3 \pm 1.4 \mathrm{~Hz}, p<0.05)$. SpC also was higher in the trained group than the control group $(30.1 \pm 1.8$ vs $24.8 \pm 1.7 \%$, $p<0.05)$. Regarding the evolution of DFr in the trained group, the values were different during VF versus the initial time of VF, being lower ( $\mathrm{p}<0.05)$, except at $180 \mathrm{~s}$.

$\mathrm{SpC}$ showed no significant differences during VF in the trained group with respect to the onset of VF.

Conclusion: Chronic exercise decreases dominant frequency and increases spectral concentration, in perfused and immersed bath, in the myocardium during ventricular fibrillation, thus decreasing the ventricular electrophysiological heterogeneity.

Chronic physical exercise, ventricular fibrillation, electrophysiological heterogeneity, spectral analysis

O2-06

HIGH FREQUENCY ELECTRICAL STIMULATION INDUCES HYPERTROPHY AND IMPROVES THE CONTRACTILE PROPERTIES OF MICE SKELETAL MUSCLE

Andresa Evelem De Melo Aroeira (1), Pedro L. Valenzuela ${ }^{(1)}$, Laura Ramírez $^{(1)}$, Joan R. Torrella ${ }^{(2)}$, Pedro De La Villa ${ }^{(1)}$

(1) Departamento de Biología de Sistemas, Facultad de Medicina Universidad de Alcalá, Madrid, Spain; ${ }^{(2)}$ Departamento de Fisiología e Inmunología, Facultad de Biología, Universidad de Barcelona, Madrid, Spain

Background: Due to the important functions of muscle mass for health (e.g. metabolism, locomotion) it is necessary to understand the physiological mechanisms inherent to the hypertrophic process as well as to develop tools to improve the musculoskeletal system and to slow the atrophic process. Most animal models used to study the hypertrophic process do not allow a precise control of the stimuli and require of negative reinforcements in the case of voluntary methods or of surgical procedures in the case of involuntary ones. It has been previously reported that high frequency electrical stimulation (EMS) induces muscle hypertrophy similarly to the active strength training, and therefore our hypothesis was that it could produce similar adaptations.

Objective: To determine the validity of high frequency EMS as an animal model to induce hypertrophy and to improve the contractile properties of skeletal muscle.

Methods: The structural, functional and biochemical changes of the Tibialis Anterior of C57BL/6J mice $(n=17)$ after 8 EMS sessions were evaluated. The left limb of the mice was stimulated whereas the right one was kept as an internal control. The muscle mass, fiber cross-sectional area and contractile and oxidative properties of both TA were compared.

Results: Our results show that EMS induced a significant $(p<0.001)$ increment of muscle mass $(19.3 \%)$ and FCSA (23.1\%), also improving contractile properties with a significant $(p<0.01)$ increase of maximal tetanic force $(20.3 \%)$ and of the rate of force development ( $23 \%$ ). EMS training did not induce any change in the oxidative capacity of skeletal muscle. 
Conclusions: Attending to the observed improvement of the muscle structure and function after EMS training, this technique should be taken into account as an animal model to study the hypertrophic process, as it enables a precise control of the stimuli (amplitude, frequency, duration, stimulation-rest ratio, etc.) and is less cruel than other methods traditionally used that require of negative reinforcement or surgical procedures.

Neuromuscular electrical stimulation, hypertrophy, animal model, muscle force, muscle mass

O3-01

\section{APOPTOTIC MECHANISMS OF ALKYNYL GOLD(I) COM- PLEXES IN COLORECTAL CARCINOMA}

Cristina Sánchez, Inés Marmol, Sonia Gascón, Jesús Osada, Ma Jesús Rodriguez-Yoldi, Elena Cerrada

Universidad de Zaragoza, Zaragoza, Spain

Cancer is one of the main causes of death and, in particular, colorectal adenocarcinoma is the second most prevalent carcinoma in Western countries. In the beginning, most chemotherapeutic agents used for cancer treatment were organic molecules, but after the discovery of the antiproliferative properties of cisplatin, a vast library of metal complexes have been synthesized as anticancer agents. In this context, gold-derivate drugs have recently emerged as new chemotherapeutic agents. Thus, here we studied the cytotoxic activity of the gold complex $[\mathrm{Au}(\mathrm{C} \equiv \mathrm{CPh})(\mathrm{PTA})]$ towards human colon cancer cell line Caco-2/TC7 through MTT assay. Then, we analyzed by flow cytometry its capacity to induce apoptosis using an Annexin V and Propidium Iodide staining. Finally we determined by flow cytometry the levels of the proapoptotic protein BAX, the anti-apoptotic protein BCL-2, cytochrome $\mathrm{c}$ and caspases 3 and 9.

We found that $[\mathrm{Au}(\mathrm{C} \equiv \mathrm{CPh})(\mathrm{PTA})]$ reduced cancer cells viability without affecting normal enterocytes. This reduction of cell viability was triggered by an induction of apoptosis whereas the contribution of necrosis was insignificant.

Once inside the cell, $[\mathrm{Au}(\mathrm{C} \equiv \mathrm{CPh})(\mathrm{PTA})]$ targets selenoproteins involved in ROS balance maintenance, triggering an increase in ROS levels as well as an alteration of anti-apoptotic and proapoptotic protein levels. The combination of these three hits stimulates the $\mathrm{Ca}^{2+}$ release from mitochondria, activation of $\mathrm{Ca}^{2+}$ dependent endonuclease(s) and oxidation of mitochondrial Permeability Transition Pore (mPTP) proteins such as VDAC, ANT and cyclophilin D. Oxidative modifications of $\mathrm{MPTP}$ proteins modify mitochondrial anion fluxes and $\mathrm{mPTP}$ reaches a high-conductance state that deregulates the entry of small solutes into the mitochondrial matrix. This phenomenon is called Mitochondrial Permeability Transition (MPT) and results in immediate dissipation of the mitochondrial membrane potential, osmotic swelling of the mitochondrial matrix and collapse of MMP resulting in the dissipation of the Mitochondrial Membrane Potential (MMP) and osmotic swelling of the mitochondrial matrix. We found that those structural changes in mitochondria stimulated the redistribution and release of cytochrome $\mathrm{c}$ from the mitochondria to the cytoplasm. In the cytoplasm, cytochrome $\mathrm{c}$ induced an increase of active caspases 9 and capsase- 3 levels in treated cells compared to control. Once capase-3 has been activated, it triggers the proteolytic cleavage of a large number of essential proteins for apoptosis. All this facts suggest that $[\mathrm{Au}(\mathrm{C} \equiv \mathrm{CPh})(\mathrm{PTA})]$ can disrupt the ROS balance and activate the mitochondrial apoptotic pathway, which will end up in a controlled cancer cell death.

Alkyne Gold(I), BCL-2, BAX, Cytocrome c, Caspases, Caco-2 cells

O3-02

TARGETING THIOREDOXIN REDUCTASE: USE OF ALKYNYL GOLD COMPLEXES IN COLORECTAL CANCER TREATMENT

Inés Mármol ${ }^{(1)}$, Cristina Sánchez ${ }^{(1)}$, Sonia Gascón ${ }^{(2)}$, Jesús Osada ${ }^{(3)}, \mathrm{M}^{\mathrm{a}}$ Jesús Rodríguez-Yoldi ${ }^{(2)}$, Elena Cerrada ${ }^{(4)}$ '

(1) Departamento de Farmacología y Fisiología, Unidad de Fisiología, Facultad de Veterinaria, Universidad de Zaragoza, Zaragoza, Spain; ${ }^{(2)}$ Departamento de Farmacología y Fisiología, Unidad de Fisiología, Facultad de Veterinaria, Universidad de Zaragoza, CIBERobn, Zaragoza, Spain; (3) Departamento de Bioquímica y Biología Molecular, Unidad de Bioquímica, Facultad de Veterinaria, Universidad de Zaragoza, CIBERobn, Zaragoza, Spain; ${ }^{(4)}$ Departamento de Química Inorgánica, Instituto de Síntesis Química y Catálisis Homogénea-ISQCH, Universidad de Zaragoza-CSIC, Zaragoza, Spain

Thioredoxin reductase (TrxR) is part of the thioredoxin system, involved in redox homeostasis. TrxR is overexpressed in some tumor cells, playing an important role in cancer development and progression. Gold derivatives such as auranofin can trigger cancer cells death by binding thioredoxin reductase because of the selenocysteine in its active site. This complex even produces an increase of reactive oxygen species (ROS) which leads to intrinsic apoptosis.

Herein, we used an alkynyl gold(I) derivative $[\mathrm{Au}(\mathrm{C} \equiv \mathrm{CPh})(\mathrm{PTA})]$ $(\mathrm{PTA}=1,3,5$-triaza-7-phosphaadamantane $)$ in order to inhibit thioredoxin reductase activity inducing cell death. Firstly we investigated changes in ROS production using the dichlorofluorescein (DCF) assay; as it was found an increase after treatment, we assumed that redox homeostasis was interrupted. Then, we investigated TrxR activity by 5,5-dithio-bis-(2-nitrobenzoic acid) (DTNB) reduction using both an enzyme produced in E. coli and from cell lysates. In each case was observed an inhibition which can correlate with previous ROS increase. We also studied the activity of glutathione reductase (GR), another enzyme involved in redox homeostasis. GR activity was measured by NADPH oxidation, and as treated cells showed no difference between mock-treated cells, it was confirmed our gold(I) derivative specificity to TrxR. Finally, we analyzed changes in TrxR mRNA expression with the purpose of elucidating cell response to treatment using qRT-PCR. We observed a surprisingly increase in TrxR expression after treatment, which was assumed as a cell mechanism for death escaping.

Alkyne gold(I), cancer, thioredoxin reductase, reactive oxygen species, Caco-2 cells. 
O3-03

ANTIBIOTIC-INDUCED DEPLETION OF MICROBIOTA INDUCES CHANGES IN THE MOTOR RESPONSE TO SEROTONIN IN MOUSE ILEUM

R. Forcén (1), E. Layunta ${ }^{(1)}$, A.C. Susunaga-Notario ${ }^{(1)}$, J.E. Mesonero (1, 2, 3), L. Grasa ${ }^{(1,2,3)}$

(1) Departamento de Farmacología y Fisiología, Facultad de Veterinaria, Universidad de Zaragoza, Zaragoza. Spain.

(2) Instituto de Investigación Sanitaria de Aragón (IIS-Aragón), Zaragoza. Spain. ${ }^{(3)}$ Instituto Agroalimentario de Aragón - IA2(Universidad de Zaragoza - CITA), Zaragoza. Spain

Background: Serotonin (5-HT) is a neurotransmitter and an important gastrointestinal signaling molecule implicated in multiple functions, such as intestinal motility. Recently, the 5-HT has been proposed to be a pro-inflammatory molecule and regulate the immune system. The actions of 5-HT on gastrointestinal motility are transduced by a large family of 5-HT receptors. Furthermore, the serotonin reuptake transporter (SERT) modulates the intestinal 5-HT availability. The gut microbiota has influence on the development of the immune system and it can be altered by different factors, for example the use of antibiotics for a long time. Intestinal dysbiosis has been associated with diseases related to gastrointestinal motor disturbances, such as Irritable Bowel Syndrome (IBS). Objectives: We evaluated the effects of the microbiota depletion evoked by the oral administration of neomycin and bacitracin on the motor response mediated by 5 -HT in mouse ileum. We also studied the gene expression of the $5-\mathrm{HT}_{2}, 5-\mathrm{HT}_{3}, 5-\mathrm{HT}_{4}$ and 5$\mathrm{HT}_{7}$ receptors, and SERT in mouse ileum.

Methods: Segments of ileum were suspended in an organ bath in the longitudinal direction of the smooth muscle cells. We studied the motor responses induced by 5-HT and fluoxetine, an inhibitor of SERT, in the ileum from mice orally treated with vehicle (sterile distilled water) or the antibiotics neomycin $(20 \mathrm{mg})$ and bacitracin $(20 \mathrm{mg})$, for 7 days. The mRNA expression levels of 5-HT receptors, and SERT in the intestine was determined by real time-PCR.

Results: Serotonin $(0.3-100 \mu \mathrm{M})$ induced a concentrationdependent contractile response, while fluoxetine (1-1000 nM) induced a concentration-dependent relaxation. Contractions evoked by 5-HT were increased in antibiotic treated mice. Relaxations evoked by fluoxetine were decreased in antibiotic treated mice respect to vehicle. The mRNA expression levels of $5-\mathrm{HT}_{3}$ receptors resulted increased, while the levels of $5-\mathrm{HT}_{2 \mathrm{~B}}, 5-\mathrm{HT}_{2 \mathrm{C}}$, $5-\mathrm{HT}_{3}, 5-\mathrm{HT}_{4}$ and $5-\mathrm{HT}_{7}$ receptors and SERT resulted decreased in antibiotic treated mice compared with vehicle.

Conclusion: These results suggest that intestinal microbiota modulates the serotoninergic system and the motor response to 5-HT in mouse ileum. Alterations in the microbiota cause an increase in the motor response induced by exogenous 5-HT, probably due to variations in the most representative 5-HT receptors implicated in the 5-HT motor response in mouse ileum. In addition, intestinal microbiota regulates the availability of endogenous 5-HT by modulating the SERT expression in mouse ileum.

SEROTONINERGIC SYSTEM, SERT, MICROBIOTA, ANTIBIOTICS, GASTROINTESTINAL MOTILITY, INTESTINE
O3-04

ACTIVATION OF TOLL-LIKE RECEPTOR 5 MODULATES THE INTESTINAL SEROTONIN TRANSPORTER IN CACO2 CELLS. COMPARATIVE STUDY WITH OTHER TOLLLIKE RECEPTORS

Eva Latorre ${ }^{(1)}$, Elena Layunta ${ }^{(2)}$, Carmen Mendoza ${ }^{(3)}$, Nyurky Matheus (3), Santiago Garcia (4), Fernando Gomollón ${ }^{(5)}$, Jose Emilio Mesonero (6)

(1) RNA - Mediated Mechanisms of Disease. University of Exeter Medical School. Exeter, Exeter, United Kingdom; (2) Departamento Farmacología y Fisiología, Facultad de Veterinaria, Instituto de Investigación Sanitaria de Aragón (IIS), Universidad de Zaragoza. Instituto Agroalimentario de Aragón IA2- (Universidad de Zaragoza - CITA), Zaragoza, Spain; (3) Departamento Ciencias Básicas. Decanato de Ciencias Veterinarias. UCLA-Venezuela, Barquisimeto, Venezuela;

(4) Servicio de Sistema Digestivo. Hospital Universitario "Miguel Servet". Instituto de Investigación Sanitaria de Aragón (IIS), Zaragoza, Spain; ${ }^{(5)}$ Servicio de Sistema Digestivo. Hospital Clínico Universitario "Lozano Blesa". Instituto de Investigación Sanitaria de Aragón (IIS), Zaragoza, Spain; ${ }^{(6)}$ Departamento Farmacología y Fisiología, Facultad de Veterinaria, Instituto de Investigación Sanitaria de Aragón (IIS), Universidad de Zaragoza. Instituto Agroalimentario de Aragón - IA2- (Universidad de Zaragoza CITA), Zaragoza, Spain

Introduction: Serotonin (5-HT) is a neuromodulator involved in the regulation of intestinal physiology, and its bioavailability is mostly determined by 5-HT uptake, carried out in the enterocyte by the serotonin transporter (SERT). Alteration of the intestinal serotonergic system is involved in gastrointestinal pathologies, like inflammatory bowel diseases (IBD), where SERT expression is modified and 5-HT levels are increased. Toll-like receptors (TLRs) recognize microorganisms and trigger innate immune responses. Deregulated TLRs activity and expression has been shown in inflamed mucosa of IBD patients. TLR5 recognizes flagellin from motile bacteria and its expression is required to maintain gastrointestinal homeostasis,

Aims \& Methods: The aim of this study was to analyze the effect of TLR5 activation on SERT activity, as a possible way to modulate the inflammatory response against flagellated intestinal bacteria. Caco-2/TC7 cell line was used as intestinal epithelial cell in vitro model. SERT activity was determined by 5-HT uptake measurement and apical-basal and basal-apical fluxes were measured using bicompartmental chambers.

Results: TLR5 activation on cells cultured in plastic support did not affect 5-HT uptake. Then, TLR5 effect was checked in cells cultured in bicompartmental chambers. Flagellin treatment at the basal border decreased 5-HT basolateral-apical flux and 5-HT accumulation; however, flagellin added to the apical border did not affect 5-HT flux. The intracellular pathway implicated in TLR5 effect was analyzed using specific inhibitors for PKA, PKC, JNK, and MEK-ERK1/2 pathways. Only PD 98059, a specific inhibitor of MEK reversed the 5-HT flux inhibition caused by flagellin.

Conclusion: Analysis of TLR5 effect on SERT activity have shown that only basal flagellin treatment reduced 5-HT basal to 
apical fluxes. Comparing the results of TLR5 activation on SERT activity, with those previously obtained after TLR3 or TLR4 activation, we can see that all of them decrease SERT activity from different cell location and using varied intracellular pathways. Thus, whereas TLR5 appears to activate the MEK-ERK1/2 intracellular pathway, TLR3 does through p38 MAPK, and TLR4 by stimulating PKC pathway. These results highlight the complex SERT regulation and the critical relationship between SERT and TLRs. This study contributes to clarify the serotonergic function under physiological and pathological conditions and how intestinal microorganisms may affect intestinal homeostasis.

In Memoriam: This work is dedicated to the late Professor Anabel Alcalde.

Supported by MICINN-ERDF (BFU2010-18971), DGA-ESF (B61), Zaragoza University (UZ2014-BIO-03), and ARAINF (2012/0567).

TLRs, SERT, 5-HT, ENTEROCYTES

\section{O3-05}

\section{INTERRELATIONSHIP BETWEEN NOD AND TLR RECEP-} TORS IN THE INTESTINAL TRACT OF MICE

Elena Layunta ${ }^{(1)}$, Eva Latorre ${ }^{(2)}$, Raquel Forcén ${ }^{(3)}$, Laura Grasa ${ }^{(1)}$, Marta Castro ${ }^{(1)}$, Julián Pardo ${ }^{(4)}$, Jose Emilio Mesonero ${ }^{(1)}$

(1) Departamento Farmacología y Fisiología, Facultad de Veterinaria, Instituto de Investigación Sanitaria de Aragón (IIS), Universidad de Zaragoza, Zaragoza. Spain. Instituto Agroalimentario de Aragón - IA2- (Universidad de Zaragoza CITA), Zaragoza, Spain., Zaragoza, Spain; ${ }^{(2)}$ RNA - Mediated Mechanisms of Disease. University of Exeter Medical School. Exeter., Exeter, United Kingdom; ${ }^{(3)}$ Departamento Farmacología y Fisiología, Facultad de Veterinaria, Instituto de Investigación Sanitaria de Aragón (IIS), Universidad de Zaragoza, Zaragoza. Spain., Zaragoza, Spain; ${ }^{(4)}$ Departamento Bioquímica y Biología Molecular y Celular, Facultad de Ciencias, Instituto de Investigación Sanitaria de Aragón (IIS), Universidad de Zaragoza, Zaragoza. Spain., Zaragoza, Spain

Introduction: The Immune system can recognize a large variety of microorganisms and their molecules through different receptors expressed in immune cells, but also in intestinal epithelial cells. TLRs and NODs are pattern recognition receptors (PPRs), which detect specific fragments that are known as microbial-or pathogenassociated molecular patterns (MAMPs or PAMPs, respectively), and are located on the cell surface or in the cytosolic compartment. Several studies have shown that these PRRs contribute to intestinal responses to microbiota and alter intestinal homeostasis/inflammation, e.g. in inflammatory bowel diseases (IBD), developing either a tolerant or a defensive response. IBD are the consequence of an incorrect defensive response to the microbiota due, in part, to the dysregulation of NODs and TLRs.

Aims \& Methods: The aim of present work was to study a possible cross-talk between NOD1 and NOD2 and both TLR2 and TLR4 in ileum and colon of mice. Control C57BL6 mice (WT), mice treated chronically with DSS (Dextran sodium sulphate), and mice deficient for TLR2 $\left(\mathrm{TLR}^{-/}{ }^{-}\right.$), TLR4 $\left(\mathrm{TLR}^{-/}{ }^{-}\right.$), and for TLR2 and TLR4 $\left(\mathrm{TLR} 2,4^{-/}\right.$) were used. Handling, equipment used and sacrifice of animals were in accordance with the European Council legislation concerning experimental protection. Ileum and colon were removed and used for RNA and protein extraction. mRNA expression was measured by real-time PCR and protein expression was quantified by western blot.

Results: The analysis of NOD1, NOD2, TLR2, and TLR4 in mice intestinal tract have shown that in ileum of DSS mice these PRRs did not seem to be modified compared with WT. In contrast, colon of DSS mice showed a NOD2 and TLR2 mRNA increased. In addition, in TLR2 $2^{-/}$mice, NOD1 expression was not modified, but NOD2 expression was increased in both ileum and colon. TLR $4^{-/}$mice showed no changes in NOD1 or NOD2 expression. However, in ileum of TLR $2,4^{-/-}$mice NOD1 was increased whereas NOD2 did not modified; on the contrary, in ileum of TLR $2,4^{-/-}$mice NOD2 expression was significantly increased and NOD1 expression resulted diminished.

Conclusion: The expression of NOD1 and NOD2 in intestinal tissue may be modulated by TLR2 and TLR4, which confirms a cross-interaction between several components of the innate immunity system. In addition, expression in DSS mice showed that only NOD2 and TLR2 are increased in colon.

In Memoriam: This work is dedicated to the late Professor Anabel Alcalde, who started this research line.

Supported by MICINN-ERDF (BFU2010-18971), DGA-ESF (B61), Zaragoza University (UZ2014-BIO-03), and ARAINF (2012/0567)

NOD1, NOD2, TLR2, TLR4, COLITIS, INTESTINE

\section{O3-06}

\section{EXPRESSION AND REGULATION OF GLUT12 IN SMALL INTESTINE AND ADIPOSE TISSUE}

\section{Eva Gil Iturbe, Carla Valriberas, $\mathrm{M}^{\mathrm{a}}$ Pilar Lostao Crespo}

Dept. Nutrition, Food Science and Physiology, University of Navarra, Pamplona, Spain; Centre for Nutrition Research, University of Navarra, Pamplona, Spain; IdiSNA, Navarra Institute for Health Research, Pamplona, Spain, Pamplona, Spain

The human facilitative glucose transporter GLUT12 was isolated from the breast cancer cell line MCF-7. It is expressed in human crude membranes of adipose tissue, small intestine and skeletal muscle, where it seems to act as a secondary insulinsensitive transporter. We have demonstrated in Xenopus laevis oocytes that hGLUT12 can transport $\alpha$-methyl-glucoside $(\alpha \mathrm{MG})$, a specific SGLT substrate, and that this transport is enhanced in the presence of $\mathrm{Na}^{+}$. GLUT12 substrate selectivity was: D-glucose $>\alpha \mathrm{MG}>$ 2-deoxy-glucose (2-DOG) $>$ Dgalactose $>$ D-fructose. Based on this information, the aim of the present work was to investigate the location and function of GLUT12 in small intestine and adipose tissue.

In small intestine, GLUT12 location was investigated by immunohistochemical methods, using an antibody anti-GLUT12 at $1: 100$, in samples fixed in bouin. GLUT12 appeared in the apical 
cytoplasm, below the brush border membrane of rat and human enterocytes; in the perinuclear region of human enterocytes and in rat basolateral cytoplasm. Western blot analysis, using the same antibody at 1:1000, demonstrated GLUT12 expression in brush border membrane vesicles of Caco- 2 cells. This expression was upregulated after $30 \mathrm{~min}$ incubation of the cells with glucose, galactose, fructose or $\alpha \mathrm{MG}$. Functional assays performed in the absence of $\mathrm{Na}^{+}$showed that $5 \mathrm{mM} \alpha \mathrm{MG}$ uptake $(15 \mathrm{~min})$ was inhibited by $50 \mathrm{mM}$ 2-DOG or fructose. Pre-incubation of Caco-2 cells with $0.1 \mu \mathrm{M}$ PMA (PKC activator) increased $5 \mathrm{mM} \alpha \mathrm{MG}$ uptake whereas pre-incubation with $10 \mathrm{mM}$ forskolin (PKA activator) did not modify it. Uptake was also increased by 10 or $25 \mathrm{ng} / \mathrm{ml}$ of TNF $\alpha$. This increase was accompanied by translocation of GLUT12 to the apical membrane.

GLUT12 location was observed in the perinuclear region of mouse white adipocytes and adipose neurons. GLUT12 was also expressed in homogenate of $3 \mathrm{~T} 3-\mathrm{L} 1$ cells. In this cell line, $0.1 \mathrm{mM}$ 2-DOG uptake in the absence of $\mathrm{Na}^{+}$was inhibited by $20 \mathrm{mM} \alpha \mathrm{MG}$, demonstrating functional expression of GLUT12. Moreover, $10 \mathrm{~min}$ pre-incubation of the cells with $50 \mathrm{nM}$ insulin increased $5 \mathrm{mM} \alpha \mathrm{MG}$ uptake.

These results demonstrate the expression, functional activity and regulation of GLUT12 in the apical membrane of enterocytes and in adipocytes, opening new perspectives to investigate its role in physiological and pathophysiological conditions in the intestine and adipose tissue.

GLUT12, Caco-2, 3 T3.L1, $\alpha \mathrm{MG}$

O4-01

THE CEREBROSPINAL FLUID OF PARKINSONIAN PATIENTS PRESENTS ELEVATED ATP13A2 CONTENT, REDUCED ATPASE ACTIVITY, AND INDUCES ATP13A2DEPENDENT RETRACTION OF NIGRAL NEURON NEURITES

Emilio Fernandez-Espejo ${ }^{(1)}$, Fatima Damas-Hermoso ${ }^{(2)}$, Jose Manuel Garcia-Moreno ${ }^{(2)}$

(1) Universidad de Sevilla, Facultad de Medicina, Sevilla, Spain;

(2) Hospital Macarena de Sevilla-SAS, Sevilla, Spain

Loss of function of ATP13A2, also known as PARK9, is causative of Kufor-Rakeb syndrome, a recessive genetic form of Parkinson's disease (PD), as well as juvenile and young-onset forms of idiopathic PD. ATP13A2 has an ATPase domain that participates in lysosomal autophagy and mitochondrial ATPase activity. ATP13A2 loss-of-function has been associated with lysosomal stress and cytotoxicity of substantia nigra neurons. The cerebrospinal fluid (CSF) is a good witness of central nervous system disturbances, and the role of CSF ATP13A2 in PD is unknown. The objectives of this study were: a) to measure ATP13A2 content and ATPase activity in PD CSF; and 2) to discern if CSF from PD patients is cytotoxic for dopamine substantia nigra neurons. CSF was obtained from PD patients and healthy controls. Informed consent forms under a protocol approved by the University of Seville and Macarena Hospital internal ethics and scientific boards were obtained from all the subjects. The findings revealed that ATP13A2 content was significantly enhanced in CSF relative to controls (ELISA, $p<0.04$; blot, $p<0.05$, Student), and that ATPase activity was found to be reduced in patients $(p<0.02)$. No correlation was found between both parameters. TH-positive nigral neurons which were exposed to Parkinsonian CSF media showed significant retraction of neurites relative to cells exposed to Neurobasal or healthy control CSF. This shrinkage effect was abolished after adding anti-ATP13A2 antibody into the culture media. To sum up, ATP13A2 is overexpressed in CSF of sporadic PD patients, a fluid that otherwise presents reduced ATPase activity. CSF from PD patients shows low-grade cytoxicity over dopamine neurons which is dependent on overexpressed ATP13A2.

Supported to EFE by Junta de Andalucia (BIO127) and Sociedad Andaluza de Neurología (SUBAIA2015/006; SAN).

ATP13A2, CSF, Parkinson, culture

O4-02

THE ROLE OF HUMORAL IMMUNE RESPONSE AND OXIDATIVE STRESS IN THE PHYSIOPATHOLOGY OF MULTIPLE SCLEROSIS

Úrsula Muñoz ${ }^{(1)}$, Cristina Sebal ${ }^{(1)}$, Esther Escudero ${ }^{(1)}$, Carolyn Sloan ${ }^{(2)}$, María De La Cruz Sádaba ${ }^{(1)}$

(1) Universidad CEU San Pablo, Madrid, Spain; ${ }^{(2)}$ John Radcliffe Hospital, Oxford, United Kingdom

The etiology of multiple sclerosis remains unknown. The main feature is the existence of inflammatory infiltrates of $\mathrm{T}$ lymphocytes, macrophages and B cells in the central nervous system. Data from our group showed that humoral immune response (IgG and $\operatorname{IgM}$ ) could play a main role in the initiation of demyelination and axonal damage. Immunoglobulin deposits in normal appearing white matter (NAWM) were detected without any further indication of inflammation. However, other authors demonstrated mitochondrial alterations and oxidative damage in lesions. Based on this background, we aimed to study the relationship between oxidative stress and humoral immune response.

Immunochemistry and immunofluorescence analyze were carried out in brain tissue samples from MS patients, and patients without neurological diseases (control group).

They were donated by University of Oxford and Universidad San Pablo CEU. To analyze immunoglobulin deposits we used antiIgG and anti-IgM antibodies EO6 antibody were used to detect oxidative stress and anti-APP antibody (Amyloid beta Precursor Protein) to analyze axon damage. TUNNEL assay was also developed to study oligodendrocyte apoptosis.

IgM or IgG deposits and oxidative stress were not detected in control group. However, we observed IgM in about (40\%), IgG and EO6 in $80 \%$ of MS patients. IgG, IgM and EO6 were present in acute, chronic active and chronic inactive lesions. We could detect $\operatorname{IgG}$ and IgM deposits but not EO6 on oligodendrocytes located in NAWM. In addition, most of TUNNEL positive oligodendrocytes showed IgG or IgM deposits. However, apoptotic oligodendrocytes were EO6 negative. IgM but not IgG or EO6 co-localized with APP on axons in demyelinating areas. 
Our work draws attention to the main role of $\operatorname{IgM}$ and $\operatorname{IgG}$ in oligodendrocyte damage. In addition, IgM is the main responsible of axon damage in patients showing IgM deposits. Further experiments will be necessary to identify the different mechanisms involved in axon damage in patients without IgM antibodies.

Multiple Sclerosis, Oxidative stress, Humoral immune response, demyelination

O4-03

ACTIONS OF THE LOCAL ANESTHETIC BENZOCAINE ON MUSCLE-TYPE NICOTINIC RECEPTORS MICROTRANSPLANTED TO THE XENOPUS OOCYTE MEMBRANE

Raúl Cobo, Armando Alberola-Die, Isabel Ivorra, Andrés Morales

Dpto. Fisiología, Genética y Microbiología. Universidad de Alicante, Alicante, Spain

Local anesthetics (LAs) are characterized by their ability to block voltage-dependent $\mathrm{Na}^{+}$-channels, and hence to preclude the action potential generation in nerve fibers. Most LAs, including lidocaine, are amine compounds which are partially protonated at physiological $\mathrm{pH}$. Recently, it has been proposed that the heterogeneity of actions of lidocaine on nicotinic acetylcholine receptors (nAChRs) are related to the presence of both the uncharged (hydrophobic) and the protonated (hydrophilic) species of this molecule in physiological solutions (Alberola-Die et al., Front. Mol. Neurosci., 9:12, 2016). By contrast, benzocaine (Bzc) is a hydrophobic LA that is not protonated at physiological $\mathrm{pH}$, in spite that it also has a tertiary amine group. Therefore, the aim of this study is to assess the effects of Bzc on nAChRs and to compare their actions with those elicited by lidocaine.

nAChRs from Torpedo electroplaques were microtransplanted to Xenopus oocytes and currents elicited by $\mathrm{ACh}\left(I_{A C h} \mathrm{~s}\right)$, either alone or with Bzc, recorded at a holding potential of $-60 \mathrm{mV}$. Coapplication of $10 \mu \mathrm{M}$ ACh with increasing concentrations of Bzc showed that Bzc reversibly blocked nAChRs, in a dose-dependent manner. The dose-inhibition curve presented an $\mathrm{IC}_{50}$ of roughly $500 \mu \mathrm{M}$ and a Hill coefficient of 1 , indicating that a single Bzc molecule accounts for the nAChR blockade. Besides, coapplication of ACh and Bzc slightly enhanced nAChR desensitization, although this effect was only evoked at high Bzc concentrations $\left(\mathrm{IC}_{50}\right.$ or higher). To assess the voltage dependence of nAChR blockade by Bzc, short voltage pulses from -120 to $+60 \mathrm{mV}$ (in $20 \mathrm{mV}$ steps) were applied during the $I_{A C h}$ plateau elicited by $10 \mu \mathrm{M}$ ACh, either alone or co-applied with $500 \mu \mathrm{M}$ Bzc. The corresponding $\mathrm{i} / \mathrm{v}$ curves showed a slight voltagedependence of the nAChR blockade by Bzc, this increasing when the oocyte was hyperpolarized, which suggests an open-channel blockade. Further evidence of Bzc molecules binding into the channel pore arises from the large current rebound elicited when Bzc was rinsed. Finally, we tested the effect of Bzc on resting nAChR by pre-applying $500 \mu \mathrm{M} \mathrm{Bzc}$, for $12 \mathrm{~s}$, just before ACh $(100 \mu \mathrm{M})$ application. Pre-application of Bzc caused a nAChR inhibition similar to that obtained by its co-application with $\mathrm{ACh}$, but the rate of $\mathrm{nAChR}$ desensitization was slowed down, instead of enhanced.

In conclusion, these results indicate that Bzc inhibits $\mathrm{nAChRs}$ by causing both open- and closed-channel blockade.

This work was partially supported by grant BFU2012-31359 from the MINECO (Spain).

Nicotinic acetylcholine receptors, Local anesthetics, Benzocaine, Xenopus oocytes, Allosteric modulation

O4-04

POSTNATAL DEVELOPMENT OF OXYGEN CONSUMPTION AND ELECTROPHYSIOLOGICAL PARAMETERS (ECG, EMG, EEG) DURING SLEEP-WAKE CYCLE IN EAR2 MICE

Ángeles Prados, Eduardo Domínguez Del Toro

Universidad Pablo de Olavide, Sevilla, Spain

Thanks to numerous researches, we know that some neurological disorders affecting considerably the population, such as ADHD, epilepsy or Parkinson's disease concern a nucleus of the brainstem: The Locus Coeruleus. This fact motivates the study of the noradrenergic system, its normal functions and how different lesions are involved in diverse diseases.

During postnatal development, noradrenergic system has been proposed as possible regulators of different processes involved in cortical maturation. Ear2 mutant mice are born and survive with the absence of more than $70 \%$ Locus Coeruleus' neurons. These mice have been demonstrated to present a functional impairment of the forebrain clock during adulthood, as well as other function like nociception, controlled by the noradrenergic projection.

Considering that this area is involved in the regulation of the sleep-wake cycle also in young animals, the aim of this work is to study how the respiratory and sleep parameters are affected by neuronal loss of the above mentioned region during early postnatal development. For such purpose, oxygen consumption, the electrical activities at the neck muscles, heart and brain of Ear2 mutant mice have been registered during the two first postnatal weeks.

Body weight and oxygen consumption (tested by an oxygen sensor during $5 \mathrm{~min}$ ) were measured daily. On the day of electrophysiological recordings, at P3, P7, P10 or P14 mice were removed from the litter. Under anaesthesia (hypothermia) the pup was implanted with two EMG hook recording electrodes aimed to the nuchal muscle, with two ECG recording electrodes in the chest and two EEG electrodes (only at P14). The electrodes were fixed to the skin with the help of Cyanoacrylate adhesive. After recovery for at least $1 \mathrm{~h}$ in a humidified incubator maintained at thermoneutrality $\left(35^{\circ} \mathrm{C}\right)$, electrodes (together with ground ones) were connected to differential amplifiers (Biopac MP35) and signals were recorded for $1 \mathrm{~h}$. EMG, ECG and EEG signals were digitized at $1 \mathrm{kHz}$ with BSL 3.7 software. The EMG and EEG 
signals for each subject helped us to distinguish between REM sleep, no-REM sleep and wakefulness.

Our results demonstrate that Locus Coeruleus' neuronal loss affects the sleep-wake cycle maturation. As a result, at P14 we observe a reduction of time spent in NoREM sleep and an increase in time spent in REM sleep, together with an increase in Heart Rate and in oxygen consumption. We conclude that noradrenergic system controls these activities during the second postnatal week.

\section{O4-05}

PRENATAL TREATMENT WITH RETINOIC ACID MODIFIES POSTNATAL SLEEP-WAKE CYCLE IN MICE

\author{
Sandra Prieto Soler, Amparo Martínez Pérez, Eduardo Domínguez \\ Del Toro
}

División de Neurociencias, Universidad Pablo de Olavide, Sevilla, Spain

During postnatal development, noradrenergic system has been proposed to play an essential role in cortical maturation. Brainstem structures organize during early embryonic development, and retinoic acid (RA) treatment on gestational day 7 (E7) has been shown to modify them, so affecting some neonatal functions, like breathing. These RA-treated mice have been demonstrated to present morphological modifications in the rostral pons, near the Locus coeruleus.

Considering that this area is involved in the regulation of the sleepwake cycle also in young animals, the aim of this work is to study how sleep parameters are affected by functional modifications at the above mentioned region during early postnatal development. For such purpose, the electrical activities at the neck muscles, heart and brain of RA-treated mice have been registered during the two first postnatal weeks.

CD-1 pregnant females were orally administered with RA $(0.5 \mathrm{mg} / \mathrm{kg}$ of maternal bodyweight dissolved in DMSO and olive oil) on E7. On the day of electrophysiological recordings, at P3, P7, P10 or P14 mice were removed from the litter. Under anaesthesia (hypothermia) the pup was implanted with two EMG hook recording electrodes aimed to the nuchal muscle, with two ECG recording electrodes in the chest and two EEG electrodes (only at P14). The electrodes were fixed to the skin with the help of Cyanoacrylate adhesive. After recovery for at least $1 \mathrm{~h}$ in a humidified incubator maintained at thermoneutrality $\left(35^{\circ} \mathrm{C}\right)$, electrodes (together with ground ones) were connected to differential amplifiers (Biopac MP35) and signals were recorded for $1 \mathrm{~h}$. EMG, ECG and EEG signals were digitized at $1 \mathrm{kHz}$ with BSL 3.7 software. The EMG and EEG signals for each subject helped us to distinguish between REM sleep, nonREM sleep and wakefulness. At P14 noradrenergic agents were administered and animals were recorded an additional hour.

Our results demonstrate that prenatal RA treatment affects the sleep-wake cycle maturation. As a result, at P14 we observe a reduction of time spent in nonREM sleep and an increase in time spent in REM sleep, together with an increase in Heart Rate. At P14 noradrenergic agents restore normality. We conclude that noradrenergic system controls these activities during the second postnatal week.

Sleep-wake cycle, heart rate, EEG, postnatal retinoic Mice

O4-06

EPIGALLOCATECHIN GALLATE AND MELATONIN ORAL TREATMENTS IMPROVE VISUAL ACUITY, CONTRAST SENSITIVITY AND ELECTRORETINOGRAM RESPONSE IN A RAT MODEL OF RETINAL DEGENERATION

Lorena Perdices $^{(1)}$, Lorena Fuentes-Broto ${ }^{(2)}$, Francisco Segura ${ }^{(1)}$, Gema Insa $^{(1)}$, Elvira Orduna ${ }^{(3)}$, Isabel Pinilla ${ }^{(4)}$

(1) Institute for Health Research of Aragón (IIS Aragón), Zaragoza, Spain; ${ }^{(2)}$ Institute for Health Research of Aragón (IIS Aragón); Department of Pharmacology and Physiology, University of Zaragoza, Zaragoza, Spain; (3) Hospital Universitario Miguel Servet, Zaragoza, Spain; (4) Institute for Health Research of Aragón (IIS Aragón); Department of Surgery, University of Zaragoza; Universitary Hospital Lozano Blesa, Zaragoza, Spain

Retinitis pigmentosa (RP) is a heterogeneous group of inherited conditions that lead to blindness. Most RP cases are due to rhodopsin mutations, which cause rod degeneration and oxidative stress. This oxidative stress may be responsible for the progressive anatomical and functional loss of the rest of retinal cells. Melatonin and epigallocatechin gallate (EGCG) have been reported to exhibit antioxidant, antiapoptotic and neuroprotective effects. Thus, we evaluated the synergistic effects of melatonin and EGCG in an animal model of RP, the $\mathrm{P} 23 \mathrm{H}$ rat.

Heterozygotes $\mathrm{P} 23 \mathrm{H}$ line 1 rats were obtained as offspring of P23H Sprague-Dawley(SD)-background crossed with Long Evans (LE) rats. In this study 20 P23HxLE were used and compared to $20 \mathrm{SD}$ crossed with LE rats (SDxLE), the reference group. Vehicle, or $10 \mathrm{mg} / \mathrm{kg} /$ day of Melatonin and/or EGCG were administered in the drinking water from 30 to 180 postnatal days. Visual function was evaluated by a monthly optomotor test that measures visual acuity (VA) and contrast sensitivity (CS) and by electroretinogram (ERG) recordings at the end of the treatment.

A decline in the ERG responses of P23HxLE rats was consistent with the gradual loss of photoreceptors. Treatment with either antioxidant increased b-wave amplitude in rats, not only in P23HxLE group but also in the reference group. VA and CS were also progressively lost with age In P23HxLE rats. Both parameters were always worse in animals with retinal degeneration than those of reference group. SDxLE rats treated with melatonin or EGCG, after 60 days of treatment ( 90 days old), maintained higher visual function parameters than those without treatment and even higher than young animals. P23HxLE rats treated with melatonin or EGCG showed better VA and CS than those treated with vehicle in all measurements done after 30 days of treatment, slowing the disease progression.

We can conclude that oral treatment of melatonin or EGCG improved vision in wild type animals and delayed vision loss in $\mathrm{P} 23 \mathrm{H}$ rats, stimulating cell function and surviving. 
Funded by Carlos III Health Institute, FIS Grant PI13/01124 Predoctoral Grant of Aragon Government (L. Perdices, F. Segura)

\section{O4-07}

SYNAPTOTAGMIN-7 CONTROLS THE SIZE OF THE RESERVE POOL OF SYNAPTIC VESICLES IN HIPPOCAMPAL NEURONS

María Angeles Montes ${ }^{(1)}$, Elisa Durán ${ }^{(1)}$, Imane Jemal ${ }^{(1)}$, Rachel Satterfield ${ }^{(2)}$, Samuel Young ${ }^{(2)}$, Guillermo Álvarez De Toledo ${ }^{(1)}$

(1) Dpto. Fisiología Médica y Biofísica. Facultad de Medicina. Universidad de Sevilla, Sevilla, Spain; ${ }^{(2)}$ Research Group Molecular Mechanisms of Synaptic Function, Max Planck Florida Institute, Florida, United States

Neurotransmitter release is a fundamental process for normal brain function, including motor, sensory, learning or behavioral activity. The lack of fine tuning of transmitter release leads to imprecise neuron to neuron communication and ultimately, to disease. Therefore, proper understanding of transmitter release might provide new insights in how we understand disease. Neurotransmitter release depends on synaptic vesicle availability, the number of vesicles fused with the presynaptic terminal and recycled during activity. We studied the role of Synaptotagmin-7 (Syt7) in the control of synaptic vesicle pools in hippocampal neurons grown in primary cultures and transfected with a genetically encoded fluorescence probe (SynaptophysinpHluorin), from wild type and knock-out mice of Syt-7. Experiments were carried out in cultures after 12 days "in vitro", in an inverted microscope equipped with epifluorescence, a monochromator and a CCD camera. Synaptic vesicles pools were determined by field stimulation at $20 \mathrm{~Hz}$. The "ready releasable pool" (RRP) was quantified by application of 40 stimuli, or the application of a sucrose pulse. The reserve pool was determined by application 600 stimuli and the resting pool by application of $\mathrm{NH}_{4} \mathrm{Cl}$. Our results show that, although Syt-7 does not participate as the fast $\mathrm{Ca}^{2+}$ sensor for neurotransmitter release as Syt-1, Syt-7 KO animals reveal an increase in the size of the reserve pool of vesicles with a proportional decrease in the resting vesicles. These data suggest that Syt7 plays a significant role in vesicle translocation from the reserve to the resting pool, making them unavailable for synaptic transmission. Also, the protein may facilitate reuse of vesicles near the plasma membrane. This effect could explain basal synaptic activity but promote depression on high levels of synaptic stimulation.

Synapse, vesicle pools, synaptotagmin, exocytosis, synaptic depression

\section{O4-08}

TRANSGENIC ALZHEIMER'S DISEASE MICE EXHIBIT VISUAL AND BEHAVIOURAL DYSFUNCTIONS

Oksana Kutsyr ${ }^{(1)}$, Pedro Lax ${ }^{(1)}$, Laura Campello ${ }^{(1)}$, Emilio De Juan $^{(1)}$, Victoria Maneu ${ }^{(1)}$, Luis Gandía ${ }^{(2)}$, Nicolás Cuenca ${ }^{(1)}$
(1) Universidad de Alicante, Alicante, Spain; ${ }^{(2)}$ Universidad Autónoma de Madrid, Madrid, Spain

Alzheimer's disease (AD) is a chronic neurodegenerative disorder characterized by progressive cognitive impairment. Besides, pathological changes underlying AD involve visual pathways. Here we aimed to assess alterations in visual and motor functions in transgenic $\mathrm{AD}$ mice (Tg-AD), which exhibit the essential pathological features of the human disease. Retinal responsiveness was evaluated by electroretinography (ERG) in Tg-AD and wild type mice. Additionally, visual acuity and contrast sensitivity were determined by optomotor test. Motor and behavioural activities were evaluated by rotarod test and spontaneous locomotor activity recording. Electroretinographic results evidenced normal scotopic $\mathrm{a}$ - and b-wave amplitudes in the Tg-AD animals as compared to controls. Oscillatory potentials and scotopic threshold responses (STR) also showed no statistically significant differences between groups. However, Tg-AD mice showed significantly lower contrast sensitivity in the optomotor test as compared to control animals. Regarding visual acuity, Tg-AD animals responded only to high-speed stimuli and with a diminished response, indicating possible deficiencies in visual motion perception. Moreover, this mouse model showed better latency time in motor tasks and a pronounced nocturnal hyperactivity. We can conclude that, in addition to motor and behavioural alterations, Tg-AD mice exhibit visual dysfunctions, as occurs in Alzheimer's disease patients.

Support: MINECO-FEDER (BFU2015-67139-R, SAF201344108-P), ISCIII-RETICS (RD12/0034/0010), FUNDALUCE, ONCE.

Neurodegeneration, electroretinogram, optomotor test, rotarod, locomotor activity

O5-01

EFFECTS OF HIGH-FAT AND HIGH-SUCROSE DIET ON VENTRICULAR REPOLARIZATION IN ISOLATED RABBIT HEART

Óscar Julián Arias-Mutis ${ }^{(1)}$, Conrado Calvo ${ }^{(2)}$, Luis Such-Miquel (3), Antonio Guill ${ }^{(2)}$, Álvaro Tormos ${ }^{(2)}$, José Millet ${ }^{(2)}$, Francisco Javier Chorro ${ }^{(1)}$, Antonio Alberola (3), Luis Such (3), Manuel Zarzoso ${ }^{(3)}$

(1) Research Foundation Clinic Hospital of Valencia (INCLIVA), Valencia, Spain; ${ }^{(2)}$ ITACA. Universitat Politècnica de València, Valencia, Spain; ${ }^{(3)}$ Universitat de València, Valencia, Spain

Introduction: Obesity and metabolic syndrome (MeS) have become a major public health concern due to its increased prevalence and link with cardiovascular disease and sudden cardiac death (SCD). Obese and diabetic patients show electrocardiographic changes that reflect a pathological remodeling associated with arrhythmias and SCD. Among those, the increase in ratecorrected QT interval and its dispersion are the most common, but the underlying mechanisms are not well understood. Our working hypothesis is that the increase in QT interval is produced by intrinsic changes in ventricular repolarization.

Methods: Twenty-five NZW rabbits were randomly assigned to a control $(n=12)$ or a MeS group $(n=13)$, fed during 28 weeks with 
high-fat (10\% hydrogenated coconut oil and $5 \%$ lard), high-sucrose (15\% dissolved in water) diet. After 28 weeks, their hearts were isolated and perfused in a Langendorff system and epicardial optical mapping was performed using two synchronized EMCCD cameras focused on the left (LV) and right (RV) ventricles. The field of view was $2.5 \times 2.5 \mathrm{~cm}(128 \times 128$ pixels $)$ and the sampling frequency 330 frames/second. Blebbistatin (7.5 $\mu \mathrm{M}$, electromechanical uncoupler) and di-4-ANBDQPQ (potentiometric dye) were used. The hearts were paced at a frequency $10 \%$ faster than sinus cycle length (SCL) and at 250, 225, 200, 175 and $150 \mathrm{~ms}$ cycle length. We analyzed, in a region of interest of $80 \times 80$ pixels, the action potential duration at $30 \%$ (APD30), $50 \%$ (APD50), $80 \%$ (APD 80 ) and $90 \%$ (APD90) of repolarization in each pacing frequency. A mixed model ANOVA was used for statistical analysis $(p<0.05)$.

Results: We did not find any modifications in APD30 and APD50 between control and MeS groups. APD80 and APD90 decreased in the RV of MeS animals $(p<0.05)$, whereas no differences were observed in the LV between groups. When comparisons were made between ventricles, APD30 and APD50 increased in the LV $(p<0.05)$ within both control and MeS groups. Regarding APD80 and APD90, we did not find differences between RV and LV.

Conclusion: High-fat and high-sucrose diet administration during 28 weeks did not modify the characteristics of ventricular repolarization in the LV, but we did find a shortening of APD80 and APD90 in the RV. Since no changes in LV repolarization were observed in isolated heart, other extrinsic nervous system or humoral influences must be implied. In addition, simultaneous optical mapping of both ventricles allowed us to find differences in early repolarization between LV and RV.

Supported by grants UV-INV-PRECOMP14-206372, GV2015062 and RD12/0042/0048 (ISCIII-FEDER funds).

Metabolic syndrome, optical mapping, ventricular repolarization, action potential duration

\section{O5-02}

\section{EARLY EVENTS IN UREMIC VASCULAR CALCIFICATION IN 5/6 NEPHRECTOMIZED RATS}

Luis Hortells, Cecilia Sosa, Ángel Millan, Víctor Sorribas

Universidad de Zaragoza, Zaragoza, Spain

Medial vascular calcification (MVC) is a complex active process of arterial wall hardening, and the most common ectopic calcification associated to chronic kidney disease (CKD)-mineral bone disorder. Our aim has been to study the very early structural and genomic changes that affect vascular smooth muscle cells (VSMC) and initiate the pathogenesis of MVC.

For this purpose, we have used 5/6 nephrectomized rats fed with a high phosphate diet $(1.2 \%)$. Aorta $\mathrm{Ca}^{2+}$ contents were quantified at different time points by colorimetry. $\mathrm{Ca} / \mathrm{P}$ deposition was studied by Scanning Electron Microscopy (SEM), and stained with Alizarin red and von Kossa for optical microscopy. Inorganic pyrophosphate (PPi) was measured by fluorimetry. Gene expressions were studied using Real Time PCR and confirmed by immunohistochemistry. Tissue-Nonspecific Alkaline Phosphatase (TNAP) activity in the artery wall was studied by NBT and fast blue RR salt staining. Several blood plasma parameter concentrations were also determined: urea, creatinine, Pi and $\mathrm{Ca}^{2+}$ (colorimetry); PPi (fluorimetry) and TNF-a, PTH, FGF-23, Klotho and Dkk1 (ELISA).

The earliest changes that we have determined in the aortic wall were increased aortic TNAP activity and Dkk1 and TNAP RNA expressions, 11 weeks after nephrectomy. Aortic $\mathrm{Ca}^{2+}$ rise and $\mathrm{Ca} /$ $\mathrm{Pi}$ deposition were only noticed after 12 weeks. Increased mRNA expressions of calcification-related genes (RUNX2, BMP2, Pit1, Pit2, HOXA10, PHOSPHO1, Fetuin-A, ANKH, OPN, ENPP1 and Klotho) were also found after the first changes. Increased plasma urea, creatinine, FGF-23, PTH and Pi were also noticed, while blood or aortic PPi were still unchanged.

Not much is known about the early stages of MVC pathogenesis, which is mandatory to determine the order of events and the development of efficacious therapies. Our results underpin a role of TNAP and Dkk1 as, most likely, the earliest players to trigger the calcification process. However, the TNAP specific role has still to be determined because PPi depletion has not been observed. On the other hand, Dkk1 could play a double role by inducing dedifferentiation and inhibiting osteodifferenciation of VSMC. The agents responsible for TNAP and Dkk1 overexpression are still unknown, but they could involve, most likely, uremic toxins and/ or growth factors that should be identified in order to provide the medical community with new pharmacological targets to treat and/ or reverse this prevalent disease.

Medial vascular calcification, Chronic kidney disease, Nephrectomized rat, Early molecular changes

O5-03

\section{MELATONIN IS AVAILABLE IN PLASMA AFTER KIDNEY} BEAN SPROUT INGESTION IN RATS

Silvia Magdalena Arribas Rodríguez (1), Pilar Rodríguez Rodríguez ${ }^{(1)}$, Ángel Luis López De Pablo León ${ }^{(1)}$, Yolanda Aguilera $^{(2)}$, Miguel Rebollo Hernanz ${ }^{(2)}$, Teresa Herrera ${ }^{(2)}$, Laura Tábata Cayuelas ${ }^{(2)}$, María De Los Ángeles Martín Cabrejas ${ }^{(2)}$

(1) Universidad Autónoma de Madrid, Madrid, Spain; ${ }^{(2)}$ CIAL, Madrid, Spain

Melatonin is a hormone with a wide range of cellular and physiological actions, including potent antioxidant properties, both as a direct free radical scavenger and through its ability to stimulate endogenous antioxidant enzymes. Melatonin is present in plants and it has been demonstrated that germination of legumes increases the plant levels of this hormone, making sprouts a suitable food source of melatonin. However, information on its bioavailability after consumption is lacking. We aimed to evaluate in rats the effect of kidney bean sprout intake on the plasma levels of melatonin and metabolically related compounds (serotonin, 6sulfatoxymelatonin), total phenolic compounds and total antioxidant capacity. In addition, we compared the plasma bioavailability derived from kidney bean sprouts versus synthetic melatonin intake. Kidney beans were germinated for 6 days and an extract was prepared in water. Male young Sprague Dawley rats were used. After weaning (day 21), the rats were kept for 23 days under changed light/dark cycle to change the pattern of melatonin secretion. The rats were administered kidney bean 
sprout extract via a gavage and blood and urine samples were obtained before and after $90 \mathrm{~min}$ of administration. The plasmatic melatonin levels increased after sprout ingestion $(16 \%, p<0.05)$. This increment correlated with the urinary 6 -sulfatoxymelatonin content, the principal biomarker of plasmatic melatonin levels $(p<0.01)$. Nevertheless, the phenolic compounds and antioxidant capacity levels did not exhibit any significant variation. The comparison of the bioavailability between the melatonin contained in the kidney bean sprouts and in a synthetic solution evidenced slightly higher levels of plasmatic melatonin $(17 \%)$ in rats fed with the solution of synthetic melatonin, but no difference in total antioxidant capacity. We conclude that: 1) melatonin is available in the plasma after kidney bean sprout ingestion, which indicates that it is readily absorbed; 2) the lack of increased plasma free radical scavenging capacity of kidney bean extract or pure melatonin is likely due to the masking effects of diet derived phenolic compounds. In summary, kidney bean sprouts could be a good source of dietary melatonin and other bioactive compounds known to have health benefits.

Acknowledgements. This work was supported by MINECO/ FEDER (FEM2012-37634-C03-0) and UAM-Santander 2015/EEUU/01.

Keywords. Melatonin, bean sprouts, plasma bioavaliability, rats

Melatonin, bean sprouts, plasma bioavaliability, rats

O5-04

FEMALE RATS ARE NOT PROTECTED FROM FETAL PROGRAMMING OF VENTRICULAR DYSFUNCTION

Pilar Rodríguez Rodríguez ${ }^{(1)}$, Ángel Luis López De Pablo ${ }^{(1)}$, Concepción Fernández García-Prieto ${ }^{(2)}$, Beatriz Somoza Hernández ${ }^{(2)}$, Begoña Quintana Villamandos ${ }^{(3)}$, José Juan Gómez De Diego ${ }^{(4)}$, Perla Yareli Gutiérrez Arzapalo ${ }^{(1)}$, Cynthia Guadalupe Reyes Hernández ${ }^{(1)}$, David Ramiro Cortijo ${ }^{(1)}$, María Del Carmen González García ${ }^{(1)}$, Silvia Magdalena Arribas Rodríguez ${ }^{(1)}$

(1) Universidad Autónoma de Madrid, Madrid, Spain; (2) Universidad San Pablo CEU, Madrid, Spain; (3) Hospital Universitario Gregorio Marañón, Madrid, Spain; ${ }^{(4)}$ Hospital Universitario San Carlos, Madrid, Spain

Background: Fetal undernutrition is a risk factor for hypertension and heart disease and has a sex-dependent pattern, being females less affected. In a rat model induced by maternal undernutrition during gestation (MUN), we have previously demonstrated that males: 1) exhibit a reduced global antioxidant capacity at weaning and 2) show increased oxidative damage and hypertension in adult age.

Hypothesis: We hypothesize that females might be protected against cardiac dysfunction through a better cardiac oxidative balance.

Material and Methods: We assessed the development of cardiac alterations, hypertension and heart oxidative stress-related enzymes in young (21 days old), adult ( 6 months old) and aged (18 months old) male and female offspring from MUN and controls rats. The rat model of maternal undernutrition (MUN) was induced by restriction of the diet to $50 \%$ of control intake during the second half of gestation, returning to ad libitum diet during lactation. In the offspring we assessed blood pressure (intra-arterial, anesthetized rats or tail-cuff); heart structure and function (histology, echocardiography) and protein expression of NADPH-oxidase, xanthine-oxidase, catalase, $\mathrm{Cu} / \mathrm{Zn}$-SOD, MnSOD, Ec-SOD (Western Blot).

Results: 21-day old MUN males were normotensive, exhibited left ventricular hypertrophy (LVH) and increased NADPHoxidase expression. Age-matched females were normotensive, without LVH and showed reduction of NADPH-oxidase and catalase expression. 6-month old MUN males were hypertensive, had smaller left ventricular mass (LVM), no differences in ejection fraction and increased xanthine-oxidase expression. Adult MUN females remained normotensive, showed smaller end-diastolic diameter, no differences in ejection fraction and lower expression of all antioxidant enzymes. 18-month old MUN males remained hypertensive, showed increased LVM and a tendency towards smaller ejection fraction. Aged MUN females remained normotensive but exhibited increased LVM and end-diastolic diameter and reduced ejection fraction.

Conclusion: We conclude that females exposed to fetal undernutrition are protected against hypertension but develop ventricular dysfunction at advanced age, even to a larger extent compared to males. Some of these alterations might be linked to disbalance in heart oxidative status.

Acknowledgements. This work was supported by MINECO/ FEDER (FEM2012-37634-C03-0 y FEM2015-63631-R).

Fetal programming, sex, heart failure, hypertension, oxidative stress

O5-05

EARLY REGRESSION OF THE THORACIC AORTA REMODELING AFTER TREATMENT WITH ESMOLOL IN AN EXPERIMENTAL RAT MODEL OF PRIMARY HYPERTENSION

Begoña Quintana-Villamandos ${ }^{(1)}$, M. Carmen González ${ }^{(2)}$, M. Jesús Delgado-Martos ${ }^{(3)}$, Luis Condezo-Hoyos ${ }^{(2)}$, Rainer H Böger ${ }^{(4)}$, Nicole Lüneburg ${ }^{(4)}$, Laia Pazó-Sayós ${ }^{(1)}$, Perla Yareli Gutiérrez-Arzapalo $^{(2)}$, Emilio Delgado-Baeza ${ }^{(3)}$

(1) Departamento Anestesiología y Reanimación Hospital General Universitario Gregorio Marañón, Madrid, Spain; ${ }^{(2)}$ Departamento Fisiología Facultad de Medicina UAM, Madrid, Spain; (3) Laboratorio Biología Molecular, Unidad de Medicina y Cirugía Experimental Hospital General Universitario Gregorio Marañón, Madrid, Spain; (4) Department of Clinical Pharmacology and Toxicology, University Medical Center Hamburg-Eppendorf, Alemania, Germany

Background: Esmolol produces early regression of left ventricular hypertrophy and improves coronary artery remodeling $[1,2]$, although the impact of short-term treatment with this beta-blocker on remodeling in thoracic aorta has not yet been studied. We hypothesized that even a short (48 h) course of esmolol might alter remodeling of the aorta in the spontaneously hypertensive rat (SHR).

Methods: Fourteen-month-old male SHRs were treated intravenously with vehicle (SHR) or esmolol (SHR-E). Age-matched, vehicle-treated male Wistar-Kyoto rats (WKY) served as controls. After $48 \mathrm{~h}$, we studied the structure, volume density of elastic fibers, and passive mechanical properties of the aorta. 
Determination of asymmetrical dimethylarginine concentrations in the aorta was analyzed.

Results: Esmolol significantly attenuated abnormal aortic wall thickness, cross-sectional area, wall-to-lumen ratio, volume density of elastic fibers, and wall stiffness.

Conclusions. Esmolol produces early regression thoracic aorta remodeling. The protective effect of esmolol could be related to a decrease in asymmetrical dimethylarginine levels.

\section{References}

1. Quintana-Villamandos B, Delgado-Martos MJ, Sánchez JJ, et al. Hypertens Res 2013; 36(5):408-13. 2. Arnalich-Montiel A, González MC, Delgado-Baeza E, et al. Biomed Res Int 2014;2014:531087.

Acknowledgements. This study was supported by a grant from the Spanish Health Ministry (Fondo de Investigaciones Sanitarias) under contract FIS 13/01261 and Fondos Feder.

Asymmetrical dimethylarginine, esmolol, hypertension, spontaneously hypertensive rats, vascular remodeling

\section{O5-06}

ROLE OF ENDOTHELIUM-DERIVED HYPERPOLARIZATION IN THE VASODILATATION OF RAT INTRARENAL ARTERIES

Estéfano Pinilla, Ana Sánchez-Pina, Mercedes Muñoz Picos, Albino García Sacristán, Dolores Prieto, Luis Rivera

Department of Physiology. Facultad de Farmacia. Universidad Complutense de Madrid, Madrid, Spain

Background and purpose: Endothelium-dependent vasodilation plays an important role in the regulation of vascular tone in different vascular beds. Besides the release of prostacyclin $\left(\mathrm{PGI}_{2}\right)$ and nitric oxide (NO), the endothelium mediates vasodilation through endothelium-derived hyperpolarization (EDH). EDH is defined as the hyperpolarizing current through myoendothelial gap junctions that hyperpolarizes and relaxes the underlying vascular smooth muscle. $\mathrm{EDH}$ has been shown to play a major role in the modulation of preglomerular tone in the renal circulation. Here, we studied the functional expression of the different $\mathrm{K}^{+}$channels involved in the $\mathrm{EDH}$ of renal arteries and its correlation with renal vasodilator responses.

Material and methods: Segmental and interlobar arteries from the kidney of male Wistar rats were used in these experiments. Freshly isolated endothelial cells were obtained by scraping the luminal surface of the artery after enzymatic digestion with trypsin, and $\mathrm{K}^{+}$currents were measured using the whole cell configuration of the patch clamp technique (Oliván-Viguera et al., Basic Clin. Pharmacol. Toxicol. Doi: 10.1111/bcpt.12560, 2016). Moreover, changes in isometric wall tension of the vessels were also studied using wire myography. Interlobar arteries were precontracted with phenylephrine (Phe) and acetylcholine (ACh) concentration-response curves were constructed under conditions of NO synthase- and cyclooxygenase blockade, in the absence and the presence of various $\mathrm{K}_{\mathrm{Ca}}$ channel blockers.

Key results: Under Voltage-clamp conditions a change in the morphology of the currents -induced by ramps from $-100 \mathrm{mV}$ to $160 \mathrm{mV} ; 600 \mathrm{~ms}-$ was observed in response to the addition of $\mathrm{ACh}$, evidencing a selective activation of channels sensitive to increases in intracellular $\mathrm{Ca}^{2+}$ concentrations $\left(\left[\mathrm{Ca}^{2+}\right]_{\mathrm{i}}\right)$. Blockers of $\mathrm{K}_{\mathrm{Ca}}$ channels, Iberiotoxin, TRAM-34 and UCL 1648, were able to inhibit different components of those currents. Moreover, in the presence of L-NAME and indomethacin, Iberiotoxin and TRAM34 inhibited ACh-induced vasodilatation in interlobar arteries mounted in wire myographs.

Conclusions and implications: The results show that $\mathrm{K}_{\mathrm{Ca}}$ channels play a major role in the $\mathrm{EDH}$ of preglomerular renal circulation. $\mathrm{BK}_{\mathrm{Ca}}, \mathrm{IK}_{\mathrm{Ca}}$ and $\mathrm{SK}_{\mathrm{Ca}}$ are functionally expressed in the endothelial cells of segmental and interlobar arteries. Stimulation of endothelial cells with $\mathrm{ACh}$ has an effect mainly increasing $\mathrm{IK}_{\mathrm{Ca}}$ currents, while basal levels of $\left[\mathrm{Ca}^{2+}\right]_{\mathrm{i}}$ may allow $\mathrm{SK}_{\mathrm{Ca}}$ currents to be active in the absence of $\mathrm{ACh}$. $\mathrm{BK}_{\mathrm{Ca}}$ and $\mathrm{IK}_{\mathrm{Ca}}$ currents have an essential role in the vasodilatation induced by $\mathrm{EDH}$, while $\mathrm{SK}_{\mathrm{Ca}}$ currents might be mainly involved in the release of NO.

Keywords: Renal arteries, $\mathrm{EDH}, \mathrm{K}_{\mathrm{Ca}}$ channels.

Renal arteries, EDH, KCa channels

O5-07

MINERALOCORTICOID RECEPTOR ANTAGONISM PREVENTS HUMAN AORTIC ENDOTHELIAL DYSFUNCTION AND MODIFIES ION CHANNEL REMODELING INDUCED BY UREMIC SERUM

Violeta Cazaña-Perez ${ }^{(1)}$, Teresa Giraldez ${ }^{(1)}$, Juan Navarro-Gonzalez ${ }^{(2)}$, Kristina Kusche-Virhog ${ }^{(3)}$, Diego Alvarez De La Rosa ${ }^{(1)}$

(1) Universidad de La Laguna, La Laguna, Spain; ${ }^{(2)}$ Hospital Universitario Nuestra Señora de Candelaria, Spain; ${ }^{(3)}$ University of Muenster, Muenster, Germany

Patients with chronic kidney disease (CKD) have a markedly increased incidence of cardiovascular events and cardiovascular disease (CVD) mortality compared with the age-matched general population. The high concentration of circulating uremic toxins in CDK patients may trigger vascular inflammatory responses, thereby inducing endothelial dysfunction, which is associated with CVD development and progression. In addition, plasma aldosterone levels are increased in CKD, and aldosterone has been found to increase vascular inflammation and fibrosis. The aim of our study was to analyze the influence of CKD in the expression of endothelial ion channels and its potential modification by mineralocorticoid receptor (MR) antagonism, which would consequently affect endothelial dysfunction. To that end we used primary human aortic endothelial cells (HAECs) cultured in medium supplemented with pooled sera from healthy subjects or patients at end-stage renal disease, as well as both groups in the presence of spironolactone. HAECs were found to express MR under the culture conditions used. Uremic serum potently up-regulated MR expression; this effect was preventable by MR antagonist spironolactone. We next have used atomic force microscopy to measure HAEC cortical stiffness, a parameter inversely correlated to endothelial nitric oxide production. Cortical stiffness was significantly increased by uremic serum suggesting functional impairment in these cells. Spironolactone prevented the increase in cortical stiffness induced by uremic serum. Since cortical stiffness is controlled by membrane potential and $\mathrm{Na}+$ influx, we used lowdensity Taqman arrays to investigate the influence of uremic serum 
and pharmacological MR antagonism on the expression of 92 ion channel subunits important in vascular physiology. We detected up-regulation of the epithelial $\mathrm{Na}+$ channel $(\mathrm{ENaC}) \alpha$ subunit by uremic serum. $\alpha \mathrm{ENaC}$ is a known transcriptional target of MR; increased $\mathrm{ENaC}$ activity promotes cortical stiffness and endothelial dysfunction, making it an ideal candidate as an MR target gene implicated in vascular adverse effects of CKD.

Chronic kidney disease, aldosterone, ENaC, mechanical stiffness, atomic force microscopy

\section{O5-08}

ROLE OF SOLUBLE ENDOGLIN IN THE PHYSIOPATHOLOGY OF PREECLAMPSIA

Lucía Pérez Roque ${ }^{(1)}$, Elena Núñez Gómez ${ }^{(1)}$, Claudia Ollauri Ibáñez ${ }^{(1)}$, Laura Ruíz Remolina ${ }^{(1)}$, Carmelo Bernabeu ${ }^{(2)}$, Fernando Pérez Barriocanal ${ }^{(1)}$, Alicia Rodríguez Barbero ${ }^{(1)}$, Miguel Pericacho ${ }^{(1)}$, José Miguel López Novoa ${ }^{(1)}$

(1) Departamento de Fisiología de la Universidad de Salamanca, Salamanca, Spain; ${ }^{(2)}$ CIB, CSIC, Madrid, Spain

Preeclampsia is a pregnancy-specific disease characterized by hypertension, proteinuria and endothelial dysfunction. It is the major cause of maternal, fetal and neonatal mortality in developing nations. Although several studies on this topic have been performed, the precise causes and the molecular pathogenesis of this disease are not completely known. Strong experimental evidence suggests that placental ischemia, resulting from the inappropriate remodeling of the maternal spiral arteries, stimulates the release of soluble factors causing hypertension and renal damage. In preeclampsia, the concentration of soluble endoglin (sEng) is increased in the maternal circulation and it has been related with the clinical manifestation and proposed as useful prognostic biomarkers. We have shown that rodents exposed to high circulating levels of sEng show severe preeclampsia-like symptoms (Valbuena-Diez et al., Circulation 2012,126:2612). The purpose of this study is to evaluate if sEng plays some role in the development of preeclampsia.

Transgenic mice overexpressing human sEng (sEng+) were generated. Wild-type (WT) female mice were crossed with sEng+ (sEng+ pregnant) or with WT male (WT pregnant). We measured plasma levels of human sEng and mice sEng (ELISA), arterial pressure (tail cuff), proteinuria, fetal mass, the histological structure of the placentas at 18th day of pregnancy, and the effect of sEng in the proliferating (MTT) and invading (transwell) ability of trophoblasts in culture.

Plasma levels of human sEng increased in plasma of sEng+ pregnant from the 11th day of pregnancy, with a peak on day 13 . Levels of mice sEng also was increased in sEng+ pregnant compared with WT pregnant. Systolic pressure was higher in sEng+ than in WT pregnant mice from the 13th day of gestation and remained increased until delivery. Proteinuria was higher in sEng+ than in control pregnant mice. Total fetal weight was lower in sEng+ than in WT pregnant mice and the number of aborts was increased in sEng+ pregnant mice than in control. Placentas from sEng+ pregnant showed marked alteration in the structure with degeneration areas, edemas and cysts. On the other hand incubation with sEng reduced the proliferation and invasiveness of cultured human trophoblasts.

In conclusion, our data suggest that soluble endoglin plays a major role in preeclampsia shown by WT female carrying sEng+ fetuses.

Soluble endoglin, preeclampsia, hypertension, placenta

O6-01

RYANODINE RECEPTOR TYPE-II ANTAGONIST VK-II-86 PREVENTS INTRINSIC ELECTROPHYSIOLOGICAL MODIFICATIONS ON REFRACTORINESS PRODUCED BY LOCAL VENTRICULAR STRETCH IN ISOLATED RABBIT HEART

Irene Del Canto ${ }^{(1)}$, Laura Santamaría ${ }^{(2)}$, Patricia Genovés ${ }^{(1)}$, Luis Such-Miquel $^{(3)}$, Manuel Zarzoso ${ }^{(3)}$, Carlos Soler ${ }^{(2)}$, Óscar Julián Arias-Mutis ${ }^{(1)}$, Antonio Alberola ${ }^{(2)}$, Francisco Javier Chorro ${ }^{(4)}$, Luis Such ${ }^{(2)}$

(1) INCLIVA, Valencia, Spain; (2) Departamento de Fisiología, Universitat de València, Valencia, Spain; ${ }^{(3)}$ Departamento de Fisioterapia, Universitat de València, Valencia, Spain; ${ }^{(4)}$ Hospital Clínico Universitario de Valencia, Universitat de València, INCLIVA, Valencia, Spain

Introduction: Acute regional ventricular stretch (ARVS) occurs under certain pathophysiological conditions, originating arrhythmogenic effects through the mechanoelectrical feedback. Mechanical effects of stretch originate calcium-related changes as sarcoplasmic recticulum $\mathrm{Ca}^{2+}$ overload that can trigger $\mathrm{Ca}^{2+}$ diastolic leaks, also known as store-overloadinduced $\mathrm{Ca}^{2+}$ release (SOICR), mediated by the cardiac ryanodine receptor (RyR2). This SOICR seems to be implicated in the mechanisms underlying stretch-induced arrhythmias. VK-II-86 (a novel selective RyR2 antagonist) acts on the intracellular $\mathrm{Ca}^{2+}$ dynamics and could modulate these effects. The aim of this investigation was to study the effects of VK-II-86 on the changes in ventricular refractoriness produced by AVRS, which is directly related with reentrant phenomenon appearance and life-threatening arrhythmias.

Methods: Eleven adult male New Zealand White rabbits (3$3.5 \mathrm{~kg}$ ) were heparinized (2500 IU) and euthanized by intravenous injection of sodium thiopental $(100 \mathrm{mg} / \mathrm{kg})$, according to European Ethic Guidelines. The hearts were excised, isolated and perfused in a Langendorff system. A pacing electrode and a recording multielectrode (121 electrodes) were placed on the left ventricle epicardium. The ARVS was produced by an "ad hoc" device, introduced into the left ventricle. The ventricular effective and functional refractory periods (VERP, VFRP) were determined by the ventricular extrastimulus test with a basic cycle length of $250 \mathrm{~ms}$, previously and at the third minute of ARVS, in control conditions and under VK-II-86 $(0,1 \mu \mathrm{M})$ infusion. The pacing threshold was determined for each situation and the stimulus amplitude was twice the diastolic threshold. An ANOVA test of repeated measures was used. Significance was reached when $p<0.05$. 
Results: Myocardial stretch reduced VERP and VFRP with respect to pre-stretch values under control conditions (VERP: 117 \pm 19 vs $97 \pm 13$ ms, VFRP: $125 \pm 17$ vs $115 \pm 12 \mathrm{~ms}, p<0.05$ ). No stretch-induced modifications of refractoriness were observed under VK-II-86 action (VERP: $111 \pm 22$ vs $115 \pm 22$ ms, VFRP: 127 \pm 18 vs $130 \pm 17 \mathrm{~ms}$, ns). Before stretching, there were no differences between control and VK-II-86 situations, whereas during stretch VERP and VFRP were significantly higher under VK-II-86 perfusion than in control conditions (VERP: $97 \pm 13$ vs 115 $\pm 22 \mathrm{~ms}$, VFRP: $115 \pm 12$ vs $130 \pm 17 \mathrm{~ms}, p<0.05$ ).

Conclusion: The ryanodine receptor type 2 antagonist, VKII-86, abolishes the intrinsic electrophysiological modifications on refractoriness produced by myocardial acute local stretch.

This work was supported by the Grant RD12/0042/0048 co-founded by the ISCIII and FEDER funds.

Cardiovascular electrophysiology, stretch, ryanodine receptor, rabbit heart

\section{O6-02}

\section{ELECTRICAL REMODELING PRODUCED IN AN EXPERI- MENTAL MODEL OF METABOLIC SYNDROME. A STUDY ON ISOLATED RABBIT HEART}

Óscar Julián Arias-Mutis ${ }^{(1)}$, Luis Such-Miquel ${ }^{(2)}$, Conrado Calvo (3), Antonio Guill (3), Álvaro Tormos (3), Germán Parra (2), Francisco Javier Chorro ${ }^{(1)}$, Antonio Alberola ${ }^{(2)}$, Luis Such ${ }^{(2)}$, Manuel Zarzoso ${ }^{(2)}$

(1) Research Foundation Clinic Hospital of Valencia (INCLIVA), Valencia, Spain; ${ }^{(2)}$ Universitat de València, Valencia, Spain; ${ }^{(3)}$ ITACA. Universitat Politècnica de València, Valencia, Spain

Introduction: The epidemic of metabolic syndrome (MeS) due to sedentary lifestyle and overconsumption of high-fat and high-sucrose diet is increasing worldwide and has been linked to an enhanced risk of cardiovascular disease and mortality. Epidemiological studies have shown that the increase in the risk of SCD is greater than the increase in the risk of myocardial infarction, suggesting that arrhythmic events are increased more than coronary events in obese and diabetic patients. Indeed, the components of MeS have been associated with an increased incidence of arrhythmias, probably related to structural and/ or electrical remodeling, but the underlying mechanisms are not completely known. Thus, our aim was to investigate the intrinsic electrical remodeling of some cardiac electrical properties produced in an experimental model of MeS.

Methods: Sixteen NZW rabbits were randomly assigned to a control $(n=8)$ or a MeS group $(n=8)$, fed during 28 weeks with high-fat $(10 \%$ hydrogenated coconut oil and $5 \%$ lard), highsucrose ( $15 \%$ dissolved in water) diet. After 28 weeks, their hearts were isolated and perfused in a Langendorff system. Pacing and recording electrodes were positioned on the left atria (LA) and ventricle (LV). Refractoriness was assessed by means of atrial and ventricular extrastimulus test with different pacing cycle lengths (10\% shorter than sinus cycle length, 250 and $200 \mathrm{~ms}$ ). We measured PP and AV intervals, and effective (ERP) and functional (FRP) refractory periods, both in LA and LV. A mixed model
ANOVA and unpaired $t$-test were used for statistical analysis $(p<0.05)$.

Results: PP interval increased in MeS group (267 \pm 28 vs 313 $\pm 40 ; p<0.05$ ) which reflected a decrease on intrinsic heart rate, whereas we did not find differences in AV interval. We found a decrease in both LA ERP and LA FRP at all the pacing frequencies in the MeS group (i.e. ERP: $95 \pm 12$ vs $77 \pm 9$ ms, FRP: $108 \pm 12$ vs $96 \pm 14 \mathrm{~ms}$ with $250 \mathrm{~ms}$ pacing cycle length; ERP: $101 \pm 14$ vs $78 \pm 9$ ms, FRP: $114 \pm 11$ vs $95 \pm 14$ ms with $200 \mathrm{~ms}$ pacing cylce length; $p<0.05$ ). We did not observe any modifications in LV refractoriness since there were no changes in LV ERP and LV FRP. Conclusion: High-fat and high-sucrose diet administration during 28 weeks produced a decrease of intrinsic heart rate and a shortening of left atrial refractoriness. As the electrophysiological modifications were exhibited in hearts not submitted to extrinsic nervous system or humoral influences, they are at least in part, intrinsic modifications.

Supported by grants UV-INV-PRECOMP14-206372, GV2015062 and RD12/0042/0048.

Metabolic syndrome, isolated heart, cardiac electrophysiology, refractoriness

O6-03

EFFECT OF CHRONIC EXERCISE ON THE ELECTRICAL STABILITY AND REFRACTORINESS OF VENTRICULAR MYOCARDIUM IN A MODEL OF ISOLATED AND FIBRILLATING RABBIT HEART

Jorge Peris ${ }^{(1)}$, Carlos Soler ${ }^{(1)}$, Manuel Zarzoso ${ }^{(1)}$, Irene Del Canto ${ }^{(2)}$, Luis Such-Miquel (1), Mariano Muñoz (1), Patricia Genovés ${ }^{(2)}$, Antonio Alberola ${ }^{(1)}$, Luis Such ${ }^{(1)}$, Francisco Javier Chorro ${ }^{(1)}$

(1) Universitat de València, Valencia, Spain; ${ }^{(2)}$ Fundación Hospital Clínico de Valencia (INCLIVA), Valencia, Spain

Introduction: The myocardium is a functional syncytium that exhibits some electrophysiological heterogeneities and electrical instability that underlie the arrhythmogenic processes. It has been reported that physical training can protect against cardiac sudden death, and it has also been proposed as an antiarrhythmic intervention, but the underlying exact mechanisms are not completely understood. We have analyzed in an isolated, perfused and fibrillating rabbit heart, the effect of physical training, on some parameters related to myocardial refractoriness, and its maintenance. We hypothesize that chronic exercise protects increasing refractoriness and maintaining this parameter.

Methods: Seven WNZ rabbits were submitted to a treadmill running program for 6 weeks and seven rabbits were housed during the same period. After training or housing, the rabbits were anaesthetized, euthanized and the hearts excised, isolated and connected to a Langendorff system. After location, the heart was submerged in a thermostatic bath $\left(37^{\circ} \mathrm{C}\right)$ in order to achieve a better maintenance of thermic conditions. A pacing electrode and a plaque with 256 recording electrodes were positioned on the left ventricle for recording. VF was triggered by pacing and maintained during $5 \mathrm{~min}$ maintaining perfusion. A VF analysis in the time domain was 
performed in order to determine the fibrillatory cycle length (VV intervals) and percentile 5 (P5) of the intervals, as a measure of ventricular refractoriness. Determinations were performed after VF onset and each minute after it, during $5 \mathrm{~min}$ in the two groups (control and trained). A two factor mixed design ANOVA with repeated measures on one factor was used for statistical analysis. When appropriate, a Bonferroni post hoc test was used. Statistical significance was considered when $p \leq 0.05$.

Results: P5 VV intervals were higher in almost all times analyzed ( $30 \mathrm{~s}, 1,2,3,4$, and $5 \mathrm{~min})$ in the trained $\left(33.1 \pm 0.5^{*}, 33.2 \pm 0.7^{*}, 34\right.$ $\pm 1^{*}, 33.85 \pm 0.96^{*}, 34.2 \pm 1.3 \#$ y $\left.33 \pm 1 \mathrm{~ms} ; n=7\right)$ than in the control group $(29.7 \pm 0.8,30.28 \pm 0.47,30.42 \pm 0.75,30.28 \pm 0.77,30 \pm 1$ and $31 \pm 1 \mathrm{~ms} ; n=7$ ). Similarly the mean value of the VV intervals was also higher in almost all times analyzed, in the trained group (52.61 $\pm 2.61^{*}, 53.5 \pm 2.4^{*}, 54.1 \pm 2.5^{*}, 54.1 \pm 1.9^{*}, 55.42 \pm 2.86$ and 53.9 $\pm 2,5 \mathrm{~ms} ; n=7)$ respect to the controls $(44.47 \pm 1.78,45.4 \pm 1.5,46.8$ $\pm 2.4,48.1 \pm 3.3,48.5 \pm 3.1$ and $49.26 \pm 3.59 \mathrm{~ms} ; n=7)$. The modifications on refractoriness parameters were maintained across the time period analyzed. ${ }^{*} p \leq 0.05$ and $\# p=0.08$ vs control.

Conclusions: It is confirmed, using our experimental model, that chronic exercise increases intrinsic ventricular refractoriness. The refractoriness modifications were maintained in time.

Chronic exercise, heart refractoriness, rabbits

\section{O6-04}

USE OF A SCORE TO ASSESS GLOBAL ANTIOXIDANT STATUS IN PLASMA AND POTENTIAL VALUE TO PREDICT PREGNANCY COMPLICATIONS

David Ramiro Cortijo ${ }^{(1)}$, Teresa Herrera ${ }^{(1)}$, Pilar Rodríguez Rodríguez ${ }^{(1)}$, Ángel Luis López De Pablo León ${ }^{(1)}$, María De La Calle ${ }^{(2)}$, María Del Rosario López Giménez ${ }^{(1)}$, Ana Isabel Mora Urda $^{(1)}$, Perla Yareli Gutiérrez Arzapalo ${ }^{(1)}$, Rubén Gómez Rioja (2), Yolanda Aguilera ${ }^{(1)}$, María Ángeles Martín Cabreja ${ }^{(1)}$, Luis Condezo Hoyos ${ }^{(1)}$, María Del Carmen González García ${ }^{(1)}$, Pilar Montero ${ }^{(1)}$, Bernardo Moreno Jiménez ${ }^{(1)}$, Silvia Magdalena Arribas Rodríguez ${ }^{(1)}$

(1) Universidad Autónoma de Madrid, Madrid, Spain; ${ }^{(2)}$ Hospital Universitario La Paz, Madrid, Spain

Background: Disbalance between Reactive Oxygen Species (ROS) and antioxidants (oxidative stress) is associated with numerous diseases, including pregnancy complications. The assessment of plasma biomarkers of is useful to predict disease development or to evaluate progression. A key question is which biomarker to assess with good predictive value. We have previously used global scores, based on the combination of multiple individual biomarkers, to evaluate oxidative stress in chronic venous insufficiency (Condezo-Hoyos et al., J Vasc Surg, 2013:57:205-213) and hypertension (Ruiz-Hurtado et al., J Hypertens 2014:32:2082-91). In the first trimester of gestation there is a physiological burst of ROS important for placentation and subsequent increase in endogenous antioxidants.

Hypothesis: Poor antioxidant status in the first trimester of pregnancy might associate with the development of a pregnancy complication; a global score based on the assessment of multiple plasma antioxidants might be useful as predictive tool.
Methods: 98 women with normal pregnancies being attended at the Obstetrics and Gynecology Service of Hospital Universitario La Paz (Madrid) were recruited. A blood sample was obtained at 10 weeks of gestation for the study of plasma biomarkers and women were followed-up at until delivery establishing two groups: Normal pregnancy and Complicated pregnancy. Plasma was used to quantify the following biomarkers: 1) carbonyls (to evaluate the degree of protein oxidative damage) and 2) antioxidants: SOD activity, catalase activity, GSH, thiol groups, polyphenols (fluorimetric and spectrophotometric micro-methods) and melatonin (ELISA). For each woman a global score of plasma antioxidant status (Antiox-S) was calculated by statistical methods (parameter normalization, standardization and sumatory of standardized antioxidants).

Results: At 10 weeks of gestation: 1) plasma carbonyl concentrations were not different between groups and were according to levels reported in the literature for normal pregnancies; 2) in women who developed a pregnancy individual antioxidants tended to be reduced complication without statistical significance, but the global parameter Antiox-S was significantly lower $(p=0.02) ; 3)$ there was a negative and significant correlation between melatonin and carbonyl levels.

Conclusions. A low global antioxidant status at the beginning of gestation associates with later development of a pregnancy complication; 2) melatonin is an important antioxidant in gestation. The global score Antiox-S might be a robust statistical tool to evaluate global antioxidant status in human populations with high biological variability. Larger population studies could help to determine the usefulness of this score as predictive tool.

Antioxidants, obstetric complications, plasma, biomarkers

\section{O6-05}

CYTOCHROME P450 (CYP)-DERIVED HYDROGEN PEROXIDE IS INVOLVED IN THE EDH-TYPE RELAXATIONS OF INTRARENAL ARTERIES

Mercedes Muñoz Picos, Elvira López-Oliva Muñoz, Estéfano Pinilla, María Pilar Martínez, Ana Alejandra Sánchez Pina, Albino García-Sacristán, Medardo Hernández Rodríguez, Luis Rivera De Los Arcos, Dolores Prieto Ocejo

\section{Universidad Complutense de Madrid, Madrid, Spain}

Healthy endothelium is an endocrine organ that contributes to vascular homeostasis by actively secreting vasoactive and trophic molecules. In response to flow-induced shear stress and to chemical signals, endothelial cells release vasodilators such as nitric oxide (NO), cyclooxygenase-derived prostacyclin $\left(\mathrm{PGI}_{2}\right)$ and the so-called endothelium-derived hyperpolarizing factor (EDHF) (Vanhoutte et al., Acta Physiol 196:193, 2009). Reactive oxygen species (ROS) like hydrogen peroxide $\left(\mathrm{H}_{2} \mathrm{O}_{2}\right)$ are involved in the in EDH-type relaxant responses of coronary and mesenteric arterioles (Matoba et al., Arterioscler Thromb Vasc Biol; 23:1224, 2003). The role of ROS in kidney vascular function has mainly been investigated in the context of harmful NADPH oxidase- and mitochondria-derived ROS generation in diabetes and obesityassociated kidney disease (Sharma, Diabetes 64:663, 2015). The present study was sought to investigate whether $\mathrm{H}_{2} \mathrm{O}_{2}$ is involved 
in the endothelium-dependent relaxations of rat intrarenal arteries as well the possible endothelial sources of ROS generation involved in these responses. Renal interlobar arteries isolated from the kidney of Wistar rats were mounted in microvascular myographs to assess function. Superoxide and $\mathrm{H}_{2} \mathrm{O}_{2}$ production were measured by chemiluminescence and fluorescence, respectively, and antioxidant enzymes (CuZn- and MnSOD) and CYP2C11 and CYP2C23 enzymes were detected by Western blotting and by inmunohistochemistry. Under conditions of cyclooxygenase and nitric oxide (NO) synthase blockade, acetylcholine (ACh) induced endothelium-dependent relaxations that were reduced in the presence of catalase suggesting the involvement of $\mathrm{H}_{2} \mathrm{O}_{2}$. ACh relaxations were also blunted by the CYP2C9 inhibitor sulfaphenazole and by the NADPH oxidase inhibitor apocynin. Acetylcholine stimulated both superoxide $\left(\mathrm{O}_{2}{ }^{-}\right)$and $\mathrm{H}_{2} \mathrm{O}_{2}$ production that were reduced by sulfaphenazole and apocynin. Levels of expression of antioxidant enzymes $\mathrm{CuZn}$ and MnSOD were similar in intrarenal arteries and renal cortex. On the other hand, exogenous $\mathrm{H}_{2} \mathrm{O}_{2}$ relaxed renal arteries by decreasing vascular smooth muscle (VSM) intracellular calcium concentration and markedly enhanced endothelial $\mathrm{K}_{\mathrm{Ca}}$ currents in freshly isolated renal endothelial cells. CYP2C11 and CYP2C23 enzymes were highly expressed in interlobar renal arteries and renal cortex, respectively, and were co-localized with eNOS in renal endothelial cells. These results demonstrate both CYP $2 \mathrm{C}$ enzymes are physiologically relevant renal endothelial source of ROS generation and that CYP-derived $\mathrm{H}_{2} \mathrm{O}_{2}$ is involved in the EDH-type relaxant responses of renal arteries.

$\mathrm{H} 2 \mathrm{O} 2$, endothelium, renal arteries, cytochrome P450 (CYP), endothelium-derived hyperpolarization (EDH)

\section{O6-06}

\section{NEMO 3.0: A SIMULATION ENVIRONMENT FOR VIRTUAL EXPERIMENTS ILLUSTRATING THE COUNTERCURRENT GAS EXCHANGE AND OXYGEN TRANSPORT ACROSS FISH GILLS}

\section{Mario Díaz, Belén Martín}

Universidad de La Laguna, La Laguna, Tenerife, Spain

Gas exchange across fish gills is a paramount example of adaptive physiology. Thanks to the appearance of countercurrent systems, where water and blood follow opposite directions, the efficiency of oxygen transport is largely improved in marine and freshwater fish. However, laboratory experiments aimed to illustrate how this particular topology functions is enormously complex and require sophisticated technologies and setups. In NEMO 3.0, we have intended to develop a simulation environment to illustrate how the countercurrent system works at the branquial level in marine and freshwater teleost fish, at the same time that different physiological parameters may be manipulated to assess the contribution of different variables, including those derived from Fick's Law. The simulation environment also allows experimental appraisals of modifications in venous oxygen concentration, blood $\mathrm{pH}$, Bohr effect, haemoglobin concentrations, fractional perfusion, effective gill area, as well as ventilation/perfusion relationships. As oxygen availability depends on environmental $\mathrm{pO}_{2}$ and $\mathrm{O}_{2}$ solubility, which, in turn is affected by temperature, salinity and $\mathrm{pH}$, we have incorporated these variables in a dedicated section of the software. Further, based on real data taken from FishBase database, we have implemented allometric relationships of representative sedentary and active fish which may be used for comparative purposes and also as model species in the simulations. NEMO 3.0 is based on a system of ' $2 n$ ' differential equations which were solved using Mathematica and implemented in a graphical interface unit using Matlab. The software, accessory files and documentation were written in Spanish and English, and are currently available for free distribution amongst academic and university communities upon request to authors.

Computer-assisted learning, fish gills, countercurrent exchange, oxygen transport

O6-07

THE LINK BETWEEN THE CLASSROOM AND THE LATEST RESEARCH: FISIOUPDATE; DEVELOPMENT OF A PROJECT FOR UPDATING SCIENTIFIC CONTENTS

Clara Azpeleta, Beatriz Gal, Elena Velarde, Francisco Javier Pardo, Almudena F. Vaquero

Universidad Europea de Madrid, Villaviciosa de Odón, Madrid, Spain

FisioUpdate is a project developed by some Lecturers teaching in courses related with Physiology at Universidad Europea de Madrid. The aim of this project is to be updated in the most recent discoveries in Physiology, and to approach this knowledge to our students in class. Since its beginning, 5 years ago, the Lecturers have attended weekly meetings, presenting and discussing 3-4 papers in each session. To date, almost 15 teachers, from 2 different Departments and different professional backgrounds, have attended permanent or occasionally, these meetings. The articles analyzed (more than 170) have dealt with varied topics, such as skeletal muscle (types of fibers, satellite cells, fatigue, exercise adaptation, hyperemia...), membrane physiology (gas transport, ionic channels...), neuromuscular junction (proteins involved, medical therapies...), brown, beige and white adipose tissue (leptin, anorexigenic hormones..) The knowledge acquired from these meetings was included and applied in subjects related to Physiology in 1st and 2nd years of many Health Sciences Degrees, such as Medicine, Sports, Physical Therapy, Psychology... Additionally, every year we have included new tools for spreading the existence of the project to other Lecturers and Departments in our University. In this sense, we have published a compendium with the summaries of the articles reviewed, and we have circulated it to our colleagues via mail. Besides, we have created a wiki space, with restricted access to the components of the FisioUpdate project, to function as a database of all the papers reviewed and a short summary of them. When appropriate, we also have uploaded to the wiki supplementary resources, such as graphs, schema, pictures or any other relevant information for a better understanding of the articles analyzed. Based on all these results, we consider that these kinds of activities are highly recommended for Lecturers, as a way to be updated in the most recent contents in a determined area. Currently, the participants on this project are highly motivated to 
continue with it, and working on the incorporation of new Lecturer for next years.

Physiology, Higher education, update, multidisciplinary

\section{O6-08}

\section{HAVE AUSCULTATION CARDIOPULMONARY AND ECHOCARDIOGRAPHY}

Lorena Franco Rubio ${ }^{(1)}$, José Carlos González ${ }^{(1)}$, Marisol Soria Aznar ${ }^{(2)}$, Jesús Escanero ${ }^{(3)}$, Manuel Guerra ${ }^{(3)}$

(1) Estudiante de Fisiología Facultad de Medicina, Zaragoza, Universidad de Zaragoza., Zaragoza, Spain; ${ }^{(2)}$ Departamento de Farmacología y Fisiología, Facultad de Ciencias de la Salud y del Deporte Huesca Universidad de Zaragoza., Huesca, Spain; ${ }^{(3)}$ Departamento de Farmacología y Fisiología. Facultad de Medicina, Zaragoza, Universidad de Zaragoza., Zaragoza, Spain

Introduction: The main purpose of this practice of physiology (Zaragoza) is not only to review the cardiac cycle but also to learn exploration techniques and to acquire an integrative vision of the cardiopulmonary systems.

Material and methods: The whole practice, which lasts for three hours, consists of four different parts:

A. First, students are able to work over the mechanism of the cardiac cycle in an interactive way, getting to draw a graphic of pressure and volume variations as well as electrocardiogram and phonocardiogram curves.

B. Afterwards the different sounds in cardiac and pulmonary auscultation are explained and heard by using a specific simulator Heart sound Lippincott.

Basic knowledge about the use of the stethoscope is given at the beginning: heart sounds, which are caused by turbulent blood flow (including the sound of the closing valves), are graded using Levine's scale from I (lowest) to VI (highest).

The most important areas to explore (mitral, tricuspid, pulmonary and aortic area) have been shown to the students, who have been able of auscultate their mates. In addition, physiological sounds, some pathological sounds such as murmurs or splits they were heard, consequently those students learn to discern between normality and dysfunction.

C. Regarding pulmonary auscultation. The practice students will be able to distinguish vesicular murmur, crackles, wheeze, rhonchi, bronchial sounds and pleural rubs using a lung simulator.

D. The last part is dedicated to echocardiograph, an echo test or heart ultrasound, taking "moving pictures" of the heart with sound waves. Afterwards an easy visual explanation of the different windows to observe the heart, volunteer students have to be explored with the Transthoracic Echocardiogram (TTE).
In long axis parasternal view, students can see left ventricle and atrium, mitral and aortic valves as well as interventricular septum, while in the short axis parasternal view right atrium and ventricle, aortic and pulmonary valves were shown.

Nevertheless the most important and useful is the apical one view, where students are able to identify the four chambers of the heart, as display the ventricular septum in the middle with the right heart displayed on the left side of the screen and the larger left heart on the right of the one. By carrying TTE out, students recognized the useful of this method to evaluate the valves and chambers of the heart from the outside of the body.

Cardiac cycle, auscultation, lung simulator, cardiac simulator, echocardiogram

O7-01

CORTISTATIN INHIBITS INSULIN SECRETION VIA SOMATOSTATIN RECEPTOR 5 IN MOUSE PANCREATIC BETA-CELLS

Sergi Soriano ${ }^{(1)}$, Alex Rafacho ${ }^{(2)}$, Paloma Alonso-Magdalena ${ }^{(3)}$, Manuel Castellano-Muñoz ${ }^{(4)}$, Eva María Bru-Tarí ${ }^{(5)}$, Beatriz Merino ${ }^{(6)}$, Antonia Ruiz ${ }^{(7)}$, Esperanza Irles ${ }^{(7)}$, Melisa Bello ${ }^{(6)}$, Pau Iborra $^{(8)}$, Sabrina Villar ${ }^{(7)}$, Jean Vetorazzi ${ }^{(9)}$, Manuel Nacher ${ }^{(10)}$, Eduard Montanya ${ }^{(11)}$, Ramón Luque ${ }^{(12)}$, Angel Nadal ${ }^{(7)}$, Ivan Quesada (7)

(1) Departament of Physiology, Genetics and Microbiology, University of Alicante, Sant Vicent del Raspeig, Spain; (2) Department of Physiological Sciences, and Multicenter Graduate Program in Physiological Sciences, Center of Biological Sciences, Federal University of Santa Catarina, 88040-900, Florianópolis, Brazil; ${ }^{(3)}$ Departamento de Biología Aplicada and CIBER de Diabetes y Enfermedades Metabólicas Asociadas (CIBERDEM), Universidad Miguel Herández, Elche, Spain; ${ }^{(4)}$ Centro de Investigación Biomédica en Red de Diabetes y Enfermedades Metabólicas Asociadas (CIBERDEM), Spain and Institute of Bioengineering, Miguel Hernández University, Elche, Spain; (5) Centro de Investigación Biomédica en Red de Diabetes y Enfermedades Metabólicas Asociadas (CIBERDEM), Spain. Institute of Bioengineering, Miguel Hernández University, Univeridad Miguel Hernández, Elche, Spain; ${ }^{6)}$ Centro de Investigación Biomédica en Red de Diabetes y Enfermedades Metabólicas Asociadas (CIBERDEM), Spain and Institute of Bioengineering, Miguel Hernández University, Elche, Spain; ${ }^{(7)}$ Centro de Investigación Biomédica en Red de Diabetes y Enfermedades Metabólicas Asociadas (CIBERDEM), Spain and Institute of Bioengineering, Miguel Hernández University, 03202, Elche, Spain., Elche, Spain; ${ }^{(8)}$ Institute of Bioengineering, Miguel Hernández University, Elche, Spain; ${ }^{(9)}$ Department of Structural and Functional Biology, Institute of Biology, Campinas State University, Campinas, Brazil; (10) Bellvitge Hospital, Barcelona, Spain; ${ }^{(11)}$ Centro de Investigación Biomédica en Red de Diabetes y Enfermedades Metabólicas Asociadas (CIBERDEM) and University of Barcelona,, Barcelona, Spain; ${ }^{(12)}$ Departamento de Biología Celular, Fisiología e Inmunología, Universidad de Córdoba, Córdoba, Spain 
Introduction: Cortistatin (CORT-14) is a neuropeptide mainly expressed in the Central Nervous System (CNS) that shows structural homology with somatostatin (SST). It was first discovered by controlling sleep physiology, but besides this central effect it displays many other actions in peripheral tissues including modulation of pancreatic islet function. However the cellular mechanism behind this phenomenon is poorly characterized.

Objectives: The aim of this work was to study the effects of CORT on electrical activity and glucose-stimulated insulin secretion (GSIS) in mouse pancreatic $\beta$-cell.

Materials and Methods: Membrane potential and single-channel recordings were analysed using patch clamp technique, calcium measurements by conventional fluorescence microscopy and hormonal secretion by ELISA.

Results: Application of 100nM CORT-14 hyperpolarized the membrane potential of pancreatic $\beta$-cell reducing glucoseinduced action potentials, calcium oscillations and insulin secretion. Single-channel analysis demonstrated that $\mathrm{K}_{\text {ATP }}$ channel was not involved in cortistatin actions. When CORT-14 was applied within the pipette recording and tolbutamide was used to block $\mathrm{K}_{\text {ATP }}$ channels, activation of small conductance channel was detected. CORT-14 effects on membrane potential and glucosestimulated insulin secretion were blocked in the presence of an antagonist of $\mathrm{G}$ protein-coupled inwardly-rectifying $\mathrm{K}^{+}$channels (GIRK) as well as in the presence of an antagonist of the somatostatin receptor type 5 (SSTR5).

Conclusions: Our results suggest that binding CORT-14 to SSTR5 receptors results in pancreatic $\beta$-cell hyperpolarization due to activation of $\mathrm{G}$ protein-coupled inwardly-rectifying $\mathrm{K}^{+}$ channels.

Funding: BFU2013-42789-P.

Cortistatin, pancreatic Beta-Cell, somatostatin receptor, GIRK channels

\section{7-02}

REGULATION OF ANGIOPOIETINS, TIE-2 AND VASCULAR ENDOTHELIAL GROWTH FACTOR BY MELATONIN IN HUMAN BREAST CANCER AND ENDOTHELIAL CELLS

Alicia González-González, Alicia González, Carolina AlonsoGonzález, Carlos Martínez-Campa, Javier Menendez-Menendez, Samuel Cos

Department of Physiology and Pharmacology, School of Medicine, University of Cantabria and Instituto de Investigación Valdecilla (IDIVAL), Santander, Cantabria, Spain

Endothelial cells represent one of the critical cellular elements in tumor microenvironment playing a crucial role in the growth and progression of cancer through control of angiogenesis. Vascular endothelial growth factor (VEGF) produced from tumor cells is essential for the expansion of breast cancer. The Angiopoietin/Tie2 family plays an important role in regulating vessel stability. Several studies emphasize the complementary and coordinated roles of angiopoietin-2 and VEGF during angiogenesis. Since melatonin regulates tumor angiogenesis by decreasing VEGF secretion and expression in tumor cells, the aim of the present study was to investigate the effects of melatonin on Ang-1, Ang-2, VEGF and tyrosine-protein kinase receptor Tie-2 expression in human breast cancer (MCF-7) and endothelial cells (HUVEC). To accomplish this we used cocultures of MCF-7 with HUVEC cells. The expression of Ang-1, Ang-2, Tie-2 and VEGF were analyzed by quantitative RT-PCR. Measurement of Ang-1, Ang-2 and VEGF protein levels in culture media was carried out by ELISA. The presence of malignant epithelial cells in the cocultures stimulated Ang-1, Ang-2 and VEGF expression and decreased Tie-2 expression in endothelial cell. Melatonin $1 \mathrm{mM}$ added to the coculture counteracted this effect and reduced Ang-1, Ang-2 and VEGF expression and increased Tie- 2 expression. In MCF-7 cells melatonin also decreased Ang-1, Ang-2 and VEGF expression and increased Tie-2 expression. The levels of Ang-1, Ang-2 and VEGF in the coculture media were increased in comparison with those found in the culture of HUVECs alone. The addition of melatonin to the cocultures counteracted the stimulatory effect induced by the presence of malignant cells on the Ang-1, Ang-2 and VEGF levels.

Estradiol (10 nM) increased Ang-1, Ang-2 and VEGF expression and melatonin counteracted the stimulatory effect induced by estradiol in HUVECs. Since tumors shift the angiogenic balance towards a pro-angiogenic state through altering the balance between the angiopoietins we analyzed the influence of melatonin in Ang-1: Ang-2 ratio. Melatonin shifted Ang-1: Ang-2 balance in favour of Ang-1.

All these results suggest that melatonin might play an important role in the paracrine interactions between malignant and endothelial cells by reducing VEGF, Ang-1 and Ang-2 expression in breast cancer and endothelial cells. This modulation of angiopoietins, coordinated with the regulation of VEGF, may be involved in the anti-angiogenic actions of melatonin and represents a new and promising therapeutic approach to the treatment of breast cancer.

This work was supported by grants from the Spanish Science Technology and Innovation Ministry (SAF2013-42012-P) and IDIVAL (APG/12).

\section{O7-03}

\section{TWO DIFFERENT HIGH-FAT DIETS PRODUCE DIFFERENT EFFECTS ON ACTIVITY/INACTIVITY CIRCADIAN RHYTHM IN RATS}

Rafael Bravo (1), Lierni Ugartemendia ${ }^{(1)}$, José María Tena ${ }^{(2)}$, Lourdes Franco ${ }^{(1)}$, Ana B. Rodríguez (1), Javier Cubero (3), Carmen Barriga ${ }^{(1)}$

(1) Department of Physiology. Faculty of Science. University of Extremadura., Badajoz, Spain; ${ }^{(2)}$ Anesthesiology, Resuscitation \& Pain Therapeutics Service. Hospital Don Benito-Villanueva., Don Benito, Spain; ${ }^{(3)}$ Experimental Science Education Area. University of Extremadura., Badajoz, Spain

Introduction: In the last two decades obesity has been studied from a chronobiological point of view. Research carried out on laboratory animals have frequently been focused in obesityinduced animals. 
Objectives: Our aim was to elucidate if two different high-fat diets produce different effects in activity/inactivity circadian rhythm. Materials \& Method: 16 wistar rats were fed a high-fat diet (35\% lipids) for 16 weeks and 42 wistar rats were fed another high-fat diet $(60 \%$ lipids) for 11 weeks. Activity/inactivity circadian rhythm was measured through actimetry both in two groups. Every variable was correlated with body weight and regression lines were compared through a slope comparison test and an intercept comparison test.

Results: Diurnal activity showed an increase in those rats fed the $35 \%$ lipids high-fat diet while rats fed the $60 \%$ lipids high-fat diet did not have any correlation with body weight so regression lines were different depending on body weight. On the other hand, nocturnal activity showed a decrease in both diets and regression lines comparison revealed they were identical depending on body weight. Related to MESOR and Amplitude both of them showed a decrease while rats body weight increased and in both cases regression lines for each diet were different. Acrophase showed a trend to get an advanced phase in $35 \%$ lipids high-fat fed rats and a trend to get a delayed phase in $60 \%$ lipids high-fat fed rats, therefore regression lines were different depending on body weight. Related to non-parametric circadian rhythm variables, interdaily stability showed a decrease and the same regression lines depending on body weight for both groups of rats, but on the other hand, intradaily variability showed an increase and different regression lines which were parallel.

Conclusion: Our results showed that both nocturnal activity and interdaily stability are modified by body weight. On the other hand diurnal activity, MESOR, amplitude, acrophase and intradaily variability were modified by diet composition.

Acknowledgments: Authors want to express their gratitude to Junta de Extremadura (Fondos FEDER: BB021- GR15051).

O7-04

SLEEP AND CIRCADIAN RHYTHM ALTERATIONS IN PATIENTS WITH RETINITIS PIGMENTOSA: EC11-441 CLINICAL TRIAL RESULTS

Lorena Fuentes-Broto ${ }^{(1)}$, Lorena Perdices ${ }^{(2)}$, Pedro Lax ${ }^{(3)}$, Gema Insa ${ }^{(2)}$, Elvira Orduna ${ }^{(4)}$, Francisco Segura ${ }^{(2)}$, Nicolás Cuenca ${ }^{(3)}$, Isabel Pinilla ${ }^{(5)}$

(1) Institute for Health Research of Aragón (IIS Aragón), Zaragoza, Spain; Department of Pharmacology and Physiology, University of Zaragoza, Zaragoza, Spain; ${ }^{(2)}$ Institute for Health Research of Aragón (IIS Aragón), Zaragoza, Spain; ${ }^{(3)}$ Department of Physiology, Genetics and Microbiology, University of Alicante, Alicante, Spain; ${ }^{(4)}$ Hospital Universitario Miguel Servet, Zaragoza, Spain; (5) Institute for Health Research of Aragón (IIS Aragón); Department of Surgery, University of Zaragoza; Universitary Hospital Lozano Blesa, Zaragoza, Spain, Zaragoza, Spain

Retinitis Pigmentosa (RP) is the most frequent cause of retinal degeneration that currently affects 1 in 3,500 people in the developed world. It is a heterogeneous group of retinal degenerative disorders with a polymorphic hereditary basis which cause a progressive loss of retinal function and represent a major cause of blindness in adult people. There is great heterogeneity in RP mechanisms depending on the mutation, however, regardless of the primary mechanism in the RP, increased oxidative stress is seeing to produce a photoreceptor retinal degeneration. This degeneration leads to loss of vision and also alters nonvisual retina functions as synchronization of biological rhythms .

Our research group is currently conducting a clinical trial with melatonin in patients over 18 years affected with Retinitis Pigmentosa in the Hospital Clinico Universitario Lozano Blesa of Zaragoza (Spain). Before the start of treatment, we have record to each patient the Pittsburgh Sleep Quality Index (PSQI) to measure the quality and patterns of sleep in adults; the Sleepiness Epworth scale to measure general level of daytime sleepiness, or their average sleep propensity in daily life; the Horne-Ostberg Questionnaire to determine morningness-eveningness in human circadian rhythms; the State-Trait Anxiety Inventory (STAI) measure anxiety at both poles of the normal affect curve (state vs. trait) and, activity, position, peripheral temperature, ambiental light and temperature by actimetry to measure the rest-activity rhythm.

These RP patients, in advanced stages, present a bad quality sleep and circadian rhythms alterations as high fragmentation, low amplitude, low circadian percentage and phase position altered in the rhythm of peripheral temperature. In the motor activity rhythm they show low circadian percentage and phase position altered.

These measures have allowed us to study objectively the alterations of sleep and circadian rhythms suffered by these patients, allowing us to evaluate the effectiveness of melatonin treatment in this clinical trial.

This clinical trial has the approval of the Ethics Committee for Clinical Research of Aragon, and the Spanish Agency for Medicines and Health Products.

Funded by the Ministry of Health, Social Services and Equality of the Government of Spain.

O7-05

MARESIN 1 INHIBITS TNF-A-INDUCED LIPOLYSIS IN 3 T3-L1 ADIPOCYTES

Laura M Laiglesia ${ }^{(1)}$, Silvia Lorente-Cebrián ${ }^{(2)}$, Miguel LópezYoldi $^{(3)}$, J Alfredo Martínez ${ }^{(4)}$, María Jesús Moreno-Aliaga ${ }^{(5)}$

(1) 1. Dept. Nutrition, Food Sciences and Physiology. School of Pharmacy and Nutrition. University of Navarra. 2. Center for Nutrition Research. University of Navarra., Pamplona, Spain; ${ }^{(2)}$ 1. Dept. Nutrition, Food Sciences and Physiology. School of Pharmacy and Nutrition. University of Navarra. 2. Center for Nutrition Research. University of Navarra. 3. Navarra Institute for Health Research (IdiSNA), Pamplona, Spain; (3) 1. Dept. Nutrition, Food Sciences and Physiology. School of Pharmacy and Nutrition. University of Navarra. 2. Center for Nutrition Research. University of Navarra., Pamplona, Spain; ${ }^{(4)} 1$. Dept. Nutrition, Food Sciences and Physiology. School of Pharmacy and Nutrition. University of Navarra. 2. Center for Nutrition Research. University of Navarra. 3. Navarra Institute for Health Research (IdiSNA). 4. CIBER Physiopathology of Obesity and Nutrition (CIBERobn), Instituto de Salud Carlos III (ISCIII)., Pamplona, Spain; ${ }^{(5)}$ 1. Dept. Nutrition, Food Sciences and Physiology. School of Pharmacy and Nutrition. University of Navarra. 2. Center for Nutrition Research. University of Navarra. 3. Navarra Institute for Health Research (IdiSNA). 4. CIBER Physiopathology of Obesity and Nutrition (CIBERobn), Instituto de Salud Carlos III (ISCIII)., Pamplona, Spain 
Obesity is associated with a chronic low-inflammatory state. Indeed, overweight and obesity are correlated to higher levels of proinflammatory cytokines such as tumour necrosis factor alpha $(\mathrm{TNF}-\alpha)$, which contributes to the inflammation of the adipose tissue. Due to an increased production of pro-inflammatory factors, obesity metabolic disorders may arise. In fact, during obesity, lipolysis levels are increased in adipocytes, and both inflammation and increased levels of free fatty acids have been involved in the development of insulin resistance. Recently, novel lipid mediators derived from omega-3 long-chain polyunsaturated fatty acids, such as maresins, protectins and resolvins have been pointed out to promote resolution of inflammation. Based on their antiinflammatory actions these lipid mediators could exert favourable actions on glucose and lipid metabolism during obesity.

The aim of the present study was to analyze the effects of Maresin 1 (MaR1) on TNF- $\alpha$-induced lipolysis in adipocytes. For this purpose, fully differentiated 3 T3-L1 adipocytes were treated with TNF- $\alpha(10-50 \mathrm{ng} / \mathrm{mL})$ in presence or absence of MaR1 $(1,10$ and $100 \mathrm{nM}$ ) for $48 \mathrm{~h}$. Glycerol released to media was evaluated using Pentra C200 autoanalyzer (Roche Diagnostic, Basel, Switzerland) and the protein expression of the main lipases and lipid dropletassociated proteins involved in the lipolytic pathway were determined by Western blot.

MaR1 inhibited TNF- $\alpha$-induced glycerol release after $48 \mathrm{~h}$, suggesting a decrease in lipolysis rate. Furthermore, MaR1 prevented the inhibition of hormone sensitive lipase (HSL) and Perilipin induced by TNF- $\alpha$. Despite the fact that MaR1 did not significantly modify the effects of TNF- $\alpha$ on adipose triglyceride lipase (ATGL) protein levels, it was capable to modulate the expression of two ATGL co-regulators (CGI58 and G0S2).

In conclusion, the current data suggest that MaR1 can attenuate the lipolytic effect of pro-inflammatory cytokines in adipocytes, which could contribute to the insulin-sensitizing properties of this specialized proresolving lipid mediator.

Maresin, Lipolysis, Obesity

\section{O7-06}

SQUALENE EFFECTS ON LIPID METABOLISM IN RABBIT Roberto Martínez-Beamonte ${ }^{(1)}$, M ${ }^{\mathrm{a}}$ José Felices $^{(2)}$, Sara Escusol ${ }^{(2)}$, Sonia Gascón ${ }^{(3)}$, Joaquín Surra ${ }^{(4)}$, Inés Mármol ${ }^{(2)}$, Jesús De La Osada ${ }^{(5)}, \mathrm{M}^{\mathrm{a}}$ Jesús Rodríguez-Yoldi ${ }^{(6)}$

(1) Departamento de Bioquímica, Unidad de Bioquímica, Facultad de Veterinaria, Universidad de Zaragoza, CIBERobn, Zaragoza, Spain; ${ }^{(2)}$ Departamento de Farmacología y Fisiología, Unidad de Fisiología, Facultad de Veterinaria, Universidad de Zaragoza, Zaragoza, Spain; ${ }^{(3)}$ Departamento de Farmacología y Fisiología, Unidad de Fisiología, Facultad de Veterinaria, Universidad de Zaragoza, CIBERobn, Zaragoza, Spain; ${ }^{(4)}$ Animal Production Units, Facultad de Veterinaria, Universidad de Zaragoza, CIBERobn, Zaragoza, Spain; ${ }^{(5)}$ Departamento de Bioquímica, Facultad de Veterinaria, Universidad de Zaragoza, CIBERobn, Zaragoza, Spain; ${ }^{(6)}$ Departamento de Farmacología y Fisiología, Unidad de Fisiología, Facultad de Veterinaria, Universidad de Zaragoza, CIBERobn, Zaragoza, Spain
Introduction: Squalene is the main unsaponificable component of virgin oive oil, and his consumption is the largest fat on Mediterranean diet, traditionally associated with a less frequency of cardiovascular diseases and lipid disorders.

Objective: The aim of this study is evaluate the differences in rabbit feed with and without squalene addition in diet, and observe the differences in lipoprotein metabolism and accumulation of lipids and cholesterol biosynthesis in liver.

Matherial and methods: Two groups of 6 male New Zealand rabbits were feed during 4 weeks with a chow diet enrichment with $1 \%$ of sunflower oil for the control group, and with $1 \%$ of sunflower oil and $0,5 \%$ of squalene for the squalene group. After this period the rabbits were fasted for $18 \mathrm{~h}$, and samples of serum and liver were obtained. The serum samples were studied for lipid content, total cholesterol and HDL cholesterol. In a second phase, we separate the different lipoproteins by FPLC for study the composition of the lipoproteins. Liver composition were analyzed for total lipids, total cholesterol and free cholesterol, and the precursors of cholesterol biosynthesis were analyzed by HPLC.

Results: The results showed that the rabbits of the squalene group have an increase of total cholesterol in serum $(p<0.001)$, with the same HDL cholesterol amount. After FPLC separation, the squalene group show an increase of VLDL and LDL free cholesterol, with a increase in APOB in these fractions too. We observe the same results in the amount of liver free cholesterol too $(p<0.05)$, with an increase of some precursors of cholesterol biosynthesis in the squalene group.

Conclusions: The enrichment of squalene diet in rabbit showed an increase of free cholesterol in VLDL and LDL lipoproteins, and in liver free cholesterol content.

Free cholesterol, squalene, lipoproteins, liver, virgin olive oil

O8-01

MOLECULAR MECHANISM OF PI DEPRIVATIONINDUCED BICARBONATURIA

Susana Lucea Arnas, Natalia Guillén Monzon, Víctor Sorribas Alejaldre

Universidad de Zaragoza, Zaragoza, Spain

Several conditions that turn into hypophosphatemia, such as dietary Pi deprivation or hyperparathyroidism, also cause renal bicarbonate wasting and decreased excretion of titratable acid and ammonia, which ends in critical disorders like metabolic acidosis, osteomalacia, etc. Interestingly, Pi deprivation and acetazolamide treatment are able to revert vascular calcification in experimental animals, most likely as a consequence of metabolic acidosis, and therefore these findings can open new therapeutic interventions for the renal patience.

Pi depletion-induced bicarbonaturia has been explained by an inhibition of the $\mathrm{Na}^{+} / \mathrm{H}^{+}$exchange, reduced proton secretion and, consequently, less titration of luminal $\mathrm{HCO}_{3}{ }^{-}$to $\mathrm{H}_{2} \mathrm{CO}_{3}$, the substrate of carbonic anhydrase. Also, a decreased ammonium transport has been reported. In our work, we have aimed to understand the molecular mechanisms of NHE inhibition, proton secretion and bicarbonaturia using the proximal tubule cell model 
Opossum Kidney (OK) cells.

OK cells were incubated overnight in DMEM with 1.0 (control) or $0.1 \mathrm{mM} \mathrm{Pi}$ (depletion) in $10 \% \mathrm{CO}_{2}$ (to keep the $\mathrm{pH}$ of medium at 7.4). ${ }^{32} \mathrm{P}$ uptake confirmed the response of the cells to Pi deprivation by increasing two times the sodium-dependent transport rate. The intracellular $\mathrm{pH}$ was decreased compared to control cells ( $1 \mathrm{mM} \mathrm{Pi})$ as measured with the $\mathrm{pH}$-sensitive dye $2^{\prime} 7^{\prime}$ bis(carboxyethyl)-5(6)-carboxyfluorescein (BCECF). The recovery to an acid load was also delayed in Pi-depleted cells.

Western blot of the NHE3 using total cell lysate did not show any change in expression abundance. The use of an antibody against the phospho-S605 NHE3 also revealed no changes. Similar results were obtained using plasma membrane proteins after cell membrane biotinylation. These results therefore are discarding the involvement of NHE3-mediated efflux of protons or $\mathrm{NH}_{4}^{+}$in bicarbonaturia. Nevertheless, a reduced ammoniagenesis as a consequence of inhibition of the phosphate-dependent glutaminase cannot be discarded.

Then, the expression of the second major proton secretor, the vacuolar-type $\mathrm{H}^{+}$-ATPase (V-ATPase) was also analyzed by western blot with the same samples. This revealed no changes in total cell protein of V-ATPase, but a clear reduction in the plasma membrane abundance of V-ATPase in OK cells. The involvement of a $\mathrm{Ba}^{2+}$-sensitive $\mathrm{K}^{+} / \mathrm{NH}_{4}^{+}$antiporter is currently under study.

In conclusion, Pi deprivation-induced bicarbonaturia seems to be a multifactorial process involving several proteins and cellular mechanisms, which need to be disclosed in order to develop safe therapies for either acidosis correction or vascular calcification treatment.

Pi deprivation, bicarbonaturia, metabolic acidosis.

O8-02

HYPERALDOSTERONISM-INDUCED HYPERTENSION AND KIDNEY INJURY ARE POTENTIATED BY EXCESS SERUM- AND GLUCOCORTICOID-INDUCIBLE KINASE 1 (SGK1) ACTIVITY IN MALE MICE

Catalina Sierra-Ramos ${ }^{(1)}$, Guadalberto Hernandez ${ }^{(1)}$, Sergio LuisLima ${ }^{(2)}$, Ana Rodriguez-Rodriguez ${ }^{(2)}$, Mercedes Muro-DeFuentes ${ }^{(3)}$, Esteban Porrini (1), Juan Navarro-Gonzalez ${ }^{(3)}$, Eduardo Salido $^{(1)}$, Diego Alvarez De La Rosa ${ }^{(1)}$

(1) Universidad de La Laguna, La Laguna, Spain; ${ }^{(2)}$ Hospital Universitario de Canarias, La Laguna, Spain; (3) Hospital Universitario Nuestra Señora de Candelaria, Santa Cruz de Tenerife, Spain

The mineralocorticoids aldosterone and deoxycorticosterone acetate (DOCA) stimulate renal tubular salt reabsorption, increase salt appetite, induce extracellular volume expansion, and elevate blood pressure. Mineralocorticoid excess induces hypertension and chronic kidney disease (CKD). The effects of mineralocorticoids on renal tubular $\mathrm{Na}^{+}$reabsorption and salt appetite involve the serum- and glucocorticoid-inducible kinase 1 (SGK1). Furthermore, SGK1 has been implicated in the development of glomerular fibrosis and inflammation. This study explored the involvement of a systemic excess of SGK1 activity in the development of mineralocorticoid/NaCl-induced hypertension and $\mathrm{CKD}$. To that end, we produced mice carrying one additional genomic copy of the mouse $s g k 1$ gene as bacterial artificial chromosome transgene (Tg.SGK1) modified with a mutation rendering the kinase constitutively active. Tg.SGK1 mice and wild-type littermates were uninephrectomized, kept with free access to standard diet and $1 \%$ saline and treated with DOCA $(75 \mathrm{mg} / \mathrm{Kg})$ for 6 weeks. DOCA $/ \mathrm{NaCl}$ treatment raised blood pressure (BP) in both genotypes, with Tg.SGK1 showing significantly higher systolic BP. To functionally evaluate renal injury, glomerular filtration rate (GFR) and urinary albumin excretion were studied by measuring iohexol plasma clearance and albumin/creatinine rate, respectively. DOCA/NaCl-induced albuminuria was significantly higher in Tg.SGK1 mice, which also showed a slight increase in GFR. Histological analysis of kidney cortex sections stained with Sirius Red revealed marked glomerular, perivascular and tubulointerstitial fibrosis in the kidney cortex, which were significantly greater in Tg.SGK1 mice. In the absence of treatment, Tg.SGK1 mice displayed enlarged glomeruli. Treatment-induced glomerular hypertrophy was further exacerbated in Tg.SGK1 mice. In order to evaluate differential gene expression between genotypes in treated animals, we performed transcriptomic analysis using Affymetrix GeneChip Analysis using kidney cortex RNA. A total of 77 significantly up-regulated and 24 significantly down-regulated genes were detected. Changes in the abundance of specific transcripts were validated by quantitative RT-PCR. This analysis uncovered collagen $27 \mathrm{~A} 1$ as a mineralocorticoid- and SGK1-target that may be involved in the development of kidney fibrosis. Our results demonstrate that increased SGK1 expression is sufficient to induce glomerular hypertrophy without added stimuli and potentiates mineralocorticoid/ $\mathrm{NaCl}$ induced hypertension and kidney injury.

Aldosterone, mineralocorticoid receptor, fibrosis

O8-03

GENDER-DEPENDENT DIFFERENCES IN THE ARTERIAL PRESSURE AND RENAL FUNCTION EFFECTS OF OBESITY AT AN EARLY AGE. ROLE OF ANGIOTENSIN II AND INTERLEUKIN-6

Antonio Tapia, Juan Manuel Moreno, F. Javier Salazar, Maria Teresa Llinás

Universidad de Murcia, IMIB, Murcia, Spain

Numerous studies have shown that obesity at an adult age is associated to a deterioration of renal function that seems to be secondary to an activation of the renin-angiotensin system and to an increase of oxidative stress. However, it is not well known to what extent obesity at early age is associated to an arterial pressure elevation and to an alteration in the regulation of renal function, and whether these changes are gender-dependent. It is also unknown the importance of angiotensin II (AngII) and oxidative stress in the arterial pressure and renal function changes 
elicited by obesity at an early age. One objective of this study was to examine the arterial pressure and renal function changes in male and female SD rats with a high fat diet from weaning to 4 months of age and to determine the role of endogenous AngII and interleukin- 6 in these changes. Another objective was to examine whether the renal sensitivity to AngII is enhanced in these rats and whether the possible greater renal sensitivity to AngII is gender-dependent. Arterial pressure was enhanced $(P<0.05)$ in male $(147 \pm 2 \mathrm{mmHg})$ and female $(137 \pm 2 \mathrm{mmHg})$ obese rats when compared to the values found in male (127 $\pm 1 \mathrm{mmHg})$ and female $(119 \pm 2 \mathrm{mmHg})$ control rats. Three days treatment with an $\mathrm{AT}_{1}$ receptor antagonist (candesartan, $7 \mathrm{mg} / \mathrm{Kg}$ ) reduced arterial pressure in obese rats $(37 \pm 4 \mathrm{mmHg}$ in males and $28 \pm 4 \mathrm{mmHg}$ in females $)(P<0.05)$ to control levels. Obesity at an early age led to an increment $(P<0.05)$ of plasma IL-6 levels in both sexes but these levels were greater $(P<0.05)$ in male $(918$ $\pm 82 \mathrm{pg} / \mathrm{ml})$ than in female $(415 \pm 64 \mathrm{pg} / \mathrm{ml})$ obese rats. The sensitivity of renal hemodynamics to Ang II was similarly enhanced in both sexes of obese rats pretreated with a converting enzyme inhibitor. The increment of renal vascular resistance elicited by Ang II in male $(106 \pm 14 \%)$ and female (106 $\pm 17 \%$ ) obese rats was greater than that found in control rats $(52 \pm 7 \%$ in males and $51 \pm 10 \%$ in females). In summary, the results of this study show that obesity at an early age induces a significant increment in arterial pressure and led to a greater renal sensitivity to Ang II in both sexes of SD rats. The elevation of arterial pressure seems to be secondary to an increase of Ang II effects and interlukin-6.

\section{O8-04}

\section{IN VITRO MODELS TO STUDY NKCC2 REGULATION BY AMPK}

Laura Martinez-Gimeno ${ }^{(1)}$, Maria Luisa Bernal-Ruiz ${ }^{(2)}$, Patricia Meade $^{(2)}$, Ignacio Gimenez ${ }^{(1)}$

(1) Instituto Aragones Ciencias Salud-IISAragon, Zaragoza, Spain;

(2) Universidad de Zaragoza, Zaragoza, Spain

The kidney specific Na-K-Cl cotransporter (NKCC2, Slc12A1 gene) is a very specialized protein with a restricted location to the apical membrane in cells of the thick ascending limb (TAL) of the Loop of Henle. NKCC2 plays a very important role in water and electrolyte homeostasis. NKCC2 is the target for loop diuretics, drugs widely-used when reducing blood pressure or extracellular volume is required. Unfortunately, heterologous expression of NKCC2 in mammal cells has proven to be very difficult. This represents a big problem for studying NKCC2 function and regulation in vitro. Here we report our efforts to develop a new in vitro cell model to study NKCC2 regulation and function.

We found that rTAL, a continuous cell line originated from rat TAL did not express NKCC2 under the conditions we used to keep them in culture. Thus, we attempted to use rTAL cells a heterologous expression system for rabbit NKCC2. A Nterminal fusion protein of red fluorescent protein mCherry with rabbit NKCC2 was transfected into rTAL cells using lipofectamine 3000. Transient expression of the fusion protein did no reach levels adequate for functional studies. Cells selected under antibiotic pressure were found to express incomplete transcripts of the fusion protein.

Next we decided to develop a method to study NKCC2 function and regulation directly in primary cell cultures of mouse TAL. We tested different strategies to isolate and grow mouse TAL primary cells. Unfortunately, we found NKCC2 expression was being lost after the first $2-4$ passages. NKCC2 expression in the first 1-2 passages, though, could be useful to perform biochemical studies of $\mathrm{NKCC} 2$ expression and regulation, by exploiting well-characterized phosphospecific antibodies for NKCC.

AMP-activated kinase (AMPK) has been demonstrated as a physiological regulator of $\mathrm{NKCC} 2$, but the relationship of this pathway to other regulatory pathways is not fully understood. TAL primary cells were grown from kidneys of mice with conditional genetic knockdown for both catalytic subunits of AMPK (AMPKalpha). Despite the loss of AMPK compared to control mice, there were not significant changes in NKCC2 expression in this mouse model.

Financial Support: Spain's Ministerio de Economía, grant DPI201128262/C02

Kidney, thick asceding limb, NKCC2, AMPK

O8-05

\section{STORAGE SYSTEMS THAT INCREASE THE LIFE OF PLATELETS}

Patricia Rego (1), Alvaro Casanova ${ }^{(2)}$, Jose Antonio Moreno (3), Pilar Peribañez (4), Daniel Falcon (4), Mercedes Galvez (3), Pedro Latorre (4), Pilar Delgado (4), Desiree Pereboom (2), Manuel Guerra (2)

(1) Estudiante de Medicina, en breve MIR anestesia, Zaragoza, Spain; ${ }^{(2)}$ Facultad de Medicina, Universidad de Zaragoza, Zaragoza Facultad de Medicina, Spain; (3) Hospital Clinico Universitario Lozano Blesa, Zaragoza, Spain; (4) Hospital Universitario Miguel Servet, Zaragoza, Spain

Introduction: Regulation of the BOE-A-2005-15514 establishes that platelet concentrates must be stored up to 5 days with continuous stirring an average temperature of $22^{\circ} \mathrm{C}$.

This fact is limited by a decrease in $\mathrm{pH}$ due to increased lactate result of anaerobic metabolic activity. If the $\mathrm{pH}$ falls below 6 irreversible morphological changes that reduce platelet viability occur. The possibility of bacterial growth is another limitation.

Platelet storage at $4{ }^{\circ} \mathrm{C}$ reduces its metabolic function, the number of contaminants but maintains stable $\mathrm{pH}$ (inhibits glycolysis). However some problems, such as increases in spontaneous aggregation, and do not recover functional activity occur. Also if the storage temperature of the concentrates is below $2{ }^{\circ} \mathrm{C}$ it is necessary to add cryoprotectors.

Objective: Get ways to increase the life of platelets and keep their viability.

Material and methods: In this study the storage method was modified through the use of three preparations:

XR2: Provides sufficient nutrients to produce energy and neutralizes the effects of lactic acid produced in glycolysis. 
XLR2: Is an inhibitor that allows blocking pathways activation of platelets added XR2.

XR3: Contains only a protector membrane.

The intervention was performed on discarded blood bank plateletrich plasma (PRP) and platelet concentrates (CP).

The samples were stored in two ways or at room temperature or frozen at $-21^{\circ} \mathrm{C}$.

The analysis of the samples stored at room temperature was carried on 6th, 7th and 8th day from storage. Analysis of frozen samples was carried at 24 and $48 \mathrm{~h}$ after thawing.

All samples were evaluated using flow cytometry:

- CELL-DYN Sapphire with measurement optical system and bioimpedance.

- Amis: was used to analyze mortality marking the samples with propidium iodide.

Results: The use of preparations (XR2, XLR2, and XR3) improves viability of platelet rich plasma and platelet concentrates after storage at room temperature and the preparations (XR2, XLR2) also after frozen storage.

Platelet Concentrates mortality platelets in the first 24 and $48 \mathrm{~h}$ of thawing is significantly decreased with the use of preparations and XR2 XLR2 over control.

The process of preserving platelets by freezing allows vital recovery levels so far not referenced in the literature.

All samples maintained with conservation methods described meet the standards of $\mathrm{pH}$ and volume required for proper storage, referenced in the literature, whether demonstrate superiority over control.

The use of XR2 and XR3 with platelet rich plasma gets a larger number of viable platelets on the eighth day, keeping them at room temperature.

Platelet, concentrates, storage, freezing, thawing, viable.

08-06

MELATONIN AS ENHANCER OF CHEMOTHERAPY TREATMENT IN HUMAN COLORECTAL ADENOCARCINOMA

Roberto Pariente, Ignacio Bejarano, José Antonio Pariente, Ana Beatriz Rodríguez, Javier Espino

Universidad de Extremadura, Badajoz, Spain

Melatonin has antitumor activity via several mechanisms including its antiproliferative and pro-apoptotic effects. Despite its potent antioxidant actions, recent evidence has indicated that melatonin may perform pro-oxidant actions in tumor cells. This study was intended to evaluate the anticancer effects of melatonin in human colorectal adenocarcinoma HT-29 cells treated with chemotherapeutic drugs.

Cells were treated with $20 \mu \mathrm{M}$ cisplatin (CIS) or1 mM 5fluorouracil (5-FU) in the absence or presence of $1 \mathrm{mM}$ melatonin. MTT assay was performed to evaluate cell citotoxicity and caspase-3activity was determined from the cleavage of its specificfluorogenic substrates. Apoptotic cell death (redistribution of phosphatidylserine in the presence of propidium iodide), activation of caspase-9, production of reactive oxygen species (ROS) and cell cycle were determined by flow cytometry.

We found that both CIS and 5-FU,incubated for 72 h,induced a decrease in HT-29 cell viability. Furthermore, melatonin significantly increased the cytotoxic effect of 5-FU. Consistently, costimulation of HT-29 cells with CIS or5-FU in the presence of melatonin further increased caspase-3 activation. Likewise, simultaneous treatments with melatonin and 5-FU significantly enhanced the ratio of cells with an overproduction of intracellular ROS and substantially augmented the population of apoptotic cells compared to the treatment with 5-FU alone. Nonetheless, melatonin only displayed moderate chemosensitizing effects in CIStreatedHT-29 cells, as suggested by a slight increment in the fraction of early apoptotic cellsthat was observed only after $48 \mathrm{~h}$. Coherently, melatonin showed an enhancing effect of the cytostatic activity of CIS as determined by cell cycle arrest.

Our findings provided evidence that in vitro melatonin strongly enhances 5-FU-induced cytotoxicity and apoptosis in HT-29 cells and, hence, the indoleamine could be potentially applied to colorectal adenocarcinoma treatment as a powerful synergistic agent. Supported by Junta de Extremadura-Fondo Social Europeo de Desarrollo Regional (FEDER) grants IB13072 and GR15051. J. Espino holds a research post-doctoral fellowship from Junta de Extremadura (jointly financed by the European Regional Development Fund (ERDF); ref. PO14011).

\section{POSTERS}

P1-01

NEUROPROTECTIVE EFFECTS IN RETINAL NEURODE-
GENERATION INDUCED BY EXCITOTOXICITY Rima Barhoum Tannous ${ }^{(1)}$, Estrella Calvo Llamas ${ }^{(2)}$, Laura Ramirez $^{(2)}$, Fernando De Abajo ${ }^{(2)}$, Pedro De La Villa Polo ${ }^{(2)}$

(1) FACULTAD DE MEDICINA. UNIVERSIDAD CEU SAN PABLO, MADRID, Spain; ${ }^{(2)}$ UNIVERSIDAD DE ALCALA, MADRID, Spain

In the physiopathology processes involved in neurodegenerative diseases can be identified two clear harmful patterns: a primary and a secondary damage neuronal degeneration (DNS) to the primary lesion. It's described several factors involved in the etiology of DNS, including excitotoxicity process, inflammation and oxidative stress. Understanding these mechanisms is essential for use of neuroprotective strategies in clinical application.

Objective: The first objective was to induce neuronal degeneration in retinal mice with intraocular administration of glutamate agonists. The second objective was to study the possible neuroprotective effect of both antagonists' calcium channel blockers (Nifedipine, Diltiazem) and agonists a-adrenergic (Brimonidine) in the retinal mice following the administration of glutamate agonists.

Material and Methods: The mouse model used was an adult of C57BL/6 J. Glutamate agonist and antagonist calcium channel administration's was by intraocular injection. Brimonidine was administered topically on the eyes of mice. Electroretinographic 
full-field binoculars and immunohistochemical were the two main methods used for functional and histological study.

Results: The electroretinographic and histological results obtained shows that calcium antagonists (Nifedipine, Diltiazem) have no neuroprotective effect on the damaged retina after intraocular administration of NMDA $30 \mathrm{mM} / \mathrm{KA} 10 \mathrm{mM}$, however, Brimonidine seems to have a neuroprotective effect after induction of the damage at concentrations of NMDA $3 \mathrm{mM} / \mathrm{KA} 1 \mathrm{mM}$.

Conclusions: The intraocular injection of high doses of glutamate agonists NMDA30mM / KA10mM causes a total loss of visual function and this damage cannot be inhibited by calcium antagonists. However, the lower NMDA3mM / KA1mM intraocular injection dose induced less structural and functional damage in the retina and this damage can be inhibited by topical administration of Brimonidine and therefore delaying the loss of visual function.

Neurodegeneration, excitotoxicity, neuroprotective, Brimonidine, calcium antagonist, electrorretinogram

P1-02

GALR2/NPYY1R HETERODIMERS INTERACT AT RECEPTOR LEVEL IN THE DENTATE GYRUS OF THE HIPPOCAMPUS IN RATS

Manuel Narváez Peláez ${ }^{(1)}$, Dasiel O. Borroto-Escuela ${ }^{(2)}$, Carmelo Millón Peñuela ${ }^{(1)}$, Antonio Flores-Burgess ${ }^{(1)}$, Belen Gago ${ }^{(1)}$, Luis Santín $^{(3)}$, Kjell Fuxe ${ }^{(2)}$, Jose Angel Narváez Bueno ${ }^{(1)}$, Zaida Díaz-Cabiale ${ }^{(1)}$

(1) Universidad de Málaga, Instituto de Investigación Biomédica de Málaga, Facultad de Medicina, Campus de Teatinos s/n, 29071 Málaga, España, Málaga, Spain; ${ }^{(2)}$ Department of Neuroscience, Karolinska Institute, Stockholm, Sweden, Estocolmo, Sweden; ${ }^{(3)}$ Universidad de Málaga, Instituto de Investigación Biomédica de Málaga, Facultad de Psicología, Campus de Teatinos s/n, 29071 Málaga, España, Málaga, Spain

Previously, we have described Galanin(GAL) and Neuropeptide $Y$ Y1(NPYY1) interactions at behavioural, cellular and receptor levels through GALR2/NPYY1R heterodimers in the amygdala. The aim of this work was to study GAL/NPYY1R interactions in the Dentate Gyrus (DG) of the Hippocampus, using autoradiographic, in situ hybridization and in situ proximity ligation assay (PLA).

Rats $(n=6)$ were sacrificed $15 \mathrm{~min}$ or $5 \mathrm{~h}$ after icv injections of GAL ( $3 \mathrm{nmol}$ ) and DG sections were incubated with NPYY1R agonist $\left[{ }^{125}\right]-\left[\mathrm{Leu}^{31}, \mathrm{Pro}^{34}\right]$ PYY $(25 \mathrm{pM})$ or NPYY1R ${ }^{33}$ PdATP specific probe, for autoradiography and in situ hybridization respectively. Autoradiograms were analyzed using NIH image analysis system and Student's unpaired $t$-test was used. For PLA, DG sections were incubated with anti-GALR2 Rabbit (1:100) and antiNPYY1R Goat (1:200). PLA signals were detected with PLA PLUS or MINUS probes for rabbit or goat/mouse antibodies. PLA signals were visualized by using a confocal microscope Leica TCS-SL confocal microscope (Leica).

We observed that GAL significant increased the NPYY1R agonist $\left[\mathrm{I}^{125}\right]-\left[\mathrm{Leu}^{31}\right.$, Pro $\left.{ }^{34}\right] \mathrm{PYY}$ binding in the DG by $20 \%(p<0,05)$ and the NPYY1R mRNA expression in the granular layer of DG by
$31 \%(p<0,001)$. Moreover, PLA-positive red clusters were found specifically in the polymorphic layer and subgranular zone of the DG. No PLA clusters were observed neither in the molecular layer of the DG nor in the corpus callosum, an area that seems to lack of GALR2 receptor.

These results demonstrate a novel mechanism of interaction between GAL and NPY1R in the DG at receptor level, probably involving the formation of GALR2/NPYY1R heteroreceptor complexes. Study supported by Junta de Andalucia CVI6476.

Galanin Receptor 2, Neuropeptide Y Receptor 1, heterodimers, Hippocampus, depression

P1-03

INFLAMMASOME MARKERS IN THE SKELETAL MUSCLE OF AN AMYOTROPHIC LATERAL SCLEROSIS MOUSE MODEL

Leticia Moreno, Laura Moreno, Miriam De La Torre, Janne Toivonen, Amaya Rando, Samanta Gasco, Pilar Zaragoza, Ana Cristina Calvo, Rosario Osta

Departamento de Anatomía, Embriología y Genética Animal, LAGENBIO-IA2, CITA, Facultad de Veterinaria, Universidad de Zaragoza, España, Zaragoza, Spain

Amyotrophic Lateral Sclerosis (ALS) is a neurodegenerative disease characterized by the atrophy and paralysis of voluntary muscles as consequence of the progressive loss of both upper and lower motor neurons. Nowadays, its etiopathogeny still remains unknown although a wide range of mechanisms that could explain this selective vulnerability of motoneurons has been proposed (oxidative stress, mitochondrial dysfunction, excitotoxicity), among which the neuroinflammation has a great relevance.

Neuroinflammation is mediated by cytosolic protein complexes known as inflammasomes, which act as intracellular sensors for infectious agents as well as for danger signals associated with neurological diseases. The best characterized is the NLRP3 inflammasome and it comprises the NLR protein NLRP3, the adaptor ASC and pro-caspase 1 . After its assembly caspase- 1 is activated, which then cleaves the precursor forms of proinflammatory cytokines IL-1 $\beta$ and IL-18 into their active forms. These cytokines, once activated and secreted, promote innate immune processes associated with infection, inflammation and autoimmunity, playing an important role in the onset of neuroinflammation and subsequent occurrence of neurodegenerative diseases, cognitive impairment and dementia. It has been observed that the NLRP3 inflammasome is implicated in numerous infectious and sterile inflammatory diseases. However, it has not been studied in depth its possible role in ALS and in particular, in a tissue such as skeletal muscle yet. For this reason, our main objective was the study of the NLRP3 inflammasome in skeletal muscle of transgenic animals SOD1G93A, a mouse model of ALS, to evaluate its involvement in the disease progression.

To achieve this objective, gene expression of the key molecular effectors of inflammasome (NLRP3, ASC, caspase-1 and IL-1 $\beta$ ) was assayed by real-time PCR in both B6SJL wild type and 
SOD1G93A mutant mice at the main stages of the disease, in order to study their potential role along disease progression in the animals. Two-tailed t-Student tests were used to assess statistical significance between groups.

Our findings showed deregulated levels of studied markers, mainly at the symptomatic stage of the disease, suggesting that the NLRP3 inflammasome can be involved in the pathogenesis of ALS. In particular, high expression level of pro-inflammatory interleukins like IL- $1 \beta$, mediated by the NLRP3 inflammasome activation, could promote an inflammatory response in this animal model.

\section{Acknowledgements}

This work was supported by Grant PI14/00947 from Fondo de Investigación Sanitaria of Spain and Consolidated Groups from Gobierno de Aragón.

Neuroinflammation, Amyotrophic Lateral Sclerosis, SOD1(G93A), NLRP3 inflammasome

\section{P1-04}

P11 MODULATES INTRINSIC EXCITABILITY AND VULNERABILITY TO EXCITOTOXIC STIMULI OF MOTONEURONS IN A MOUSE MODEL OF AMYOTROPHIC LATERAL SCLEROSIS (ALS)

Victoria García Morales ${ }^{(1)}$, Federico Portillo ${ }^{(1)}$, Laura Gómez Pérez ${ }^{(2)}$, Lucía Molanes Pérez ${ }^{(1)}$, Bernardo Moreno López ${ }^{(1)}$

(1) Facultad de Medicina (Universidad de Cádiz), Cádiz, Spain; (2) Emory University School of Medicine, Cádiz, United States

Increase of intrinsic excitability (increased membrane resistance $(\mathrm{Rm})$ and membrane potential $(\mathrm{Vm})$ depolarization) in neurons might exacerbate intracellular $\mathrm{Ca}^{2+}$ mobilization triggered by excitatory neurotransmitters and lead to loss on neuronal function and subsequent death. Glutamate (Glut)-induced excitotoxicity is a major pathogenic mechanism involved in motor neuron $(\mathrm{MN})$ degeneration at several motor pathologies. Thus, understanding molecular mechanisms involved in the regulation of intrinsic excitability of MNs has a basic and clinical interest. In this line, $\mathrm{p} 11$, a retention factor in reticulum endoplasmic for TASK1 (leak $\mathrm{K}^{+}$channels), could be a key regulator of excitability and hypersensibilization against excitotoxic stimuli of MNs. Pretreatment of primary cultures of embryonic spinal MNs (SMNs) with an small interfering RNA against mRNA for $\mathrm{p} 11\left(\mathrm{siRNA}_{\mathrm{p} 11}\right)$, induced both, a reduction in intrinsic excitability of SMNs (Rm decrease, Vm hyperpolarization) and an increase in survival of SMNs exposedto anexcitotoxic estimulus (Glut $150 \mu \mathrm{M}, 30 \mathrm{~min}$ ) relative to control RNA (cRNA). These effects were TASK1-dependent (absent in SMNs fromtask1 ${ }^{-/}$). p11 up-regulation and TASK1 subunits down-regulation were observed in the spinal cord extracted from the ALS mouse model SOD1 ${ }^{\mathrm{G} 93 \mathrm{~A}}$.p11 deregulation was also observed in SMNs ${ }^{\text {SOD1G93A }} \cdot$ siRNA $_{\text {p11 }}$ attenuated hyperexcitability observed in SMNs ${ }^{\text {SOD1G93A }}$ and reduced vulnerability against Glut. By means of imaging procedures, it was observed that siRNA $\mathrm{p}_{\mathrm{p} 11}$ reduced basal $\left[\mathrm{Ca}^{2+}\right]_{\text {int }}$, attenuated $\left[\mathrm{Ca}^{2+}\right]_{\text {int }}$ increase triggered by Glut and delayed Glut-induced $\left[\mathrm{Ca}^{2+}\right]_{\text {int }}$ deregulationin SMNs and

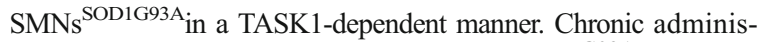
tration of siRNA ${ }_{p 11}$ to pre-symptomatic SOD $1^{\mathrm{G} 93 \mathrm{~A}}$ mice delayed motor deficits onset, increased the number of surviving $\mathrm{MNs}$ in the sacro-lumbar segment, and increased their life spam. Altogether these outcomes indicate a pivotal role of $\mathrm{p} 11$ in the regulation of neuronal excitability via TASK1 subunits, then modulating $\left[\mathrm{Ca}^{2+}\right]_{\text {int }}$ dynamics. The neuroprotective effect observed of reducing p11 levels, which wasTASK1-dependent, suggests that such as TASK1 openersas modulators of p11 expression could be potentially interesting therapeutic strategies in the treatment of several MNs pathologies.

Financial support: MINECO/FEDER (SAF2011-23633; BFU2015-71422-R), CEIC Junta de Andalucía (PAI011CTS-7281).

Motoneurons, excitotoxicity, excitability, TASK channels, p11

P1-05

MULTIMEDIA: A USEFUL TOOL FOR IMPROVING THE LEARNING OF PHYSIOLOGY

Solanye Guerra-Ojeda ${ }^{(1)}$, Eva Serna ${ }^{(2)}$, Soraya Lilian Vallés ${ }^{(1)}$, Martin Aldasoro ${ }^{(1)}$, Jose Vila ${ }^{(1)}$, Maria Dolores Mauricio ${ }^{(1)}$

${ }^{(1)}$ Departamento de Fisiología, Universidad de Valencia, Valencia, Spain; ${ }^{(2)}$ Departamento de Patología, Universidad de Valencia, Valencia, Spain

Introduction: Teaching in Physiology is delivery through thematic units to improve the learning of different topics, but we found that most of the students have difficulties to connect units together and get an overview of the subject. Given the importance of the integration of physiological processes, we believe that methods that allow students to interrelate the functions of different physiological systems help to enhance learning of physiology.

Objectives: The main goal of our project was to integrate two major thematic units of human physiology, the cardiovascular and endocrine system, by developing multimedia material. The material was developed as a research project, with the additional objective to introduce students to scientific method.

Methodology: The development of the multimedia material was supported by the grant "Projectes Innovació Educativa 2015-2016, University of Valencia". At the beginning of the activity we proposed a working hypothesis. The students had to evaluate if the hormonal changes during the menstrual cycle could modify the values of blood flow and their impact on regulatory mechanisms. In order to test this hypothesis, blood flow in the forearm was indirectly measured by venous occlusion plethysmography.

Results: The multimedia material is available to students on: http://mmedia.uv.es/html5/g/cream//45327_pletismografia_cas. mp4. It is divided in three parts; the first one contents an introduction to menstrual cycle and the relationship between estrogen and endothelium, the hypothesis and the experimental design protocol. The second part shows the use of the 
plethysmograph and the third part describes how to interpret results, explaining the physiological mechanisms in which the technique is based, and finally, the conclusion of the study.

Conclusions: The development of multimedia material gives students a novel approach to physiology as a basis for biomedical research. It helps students to reason and not to learn in a rote manner. The activity also reinforces understanding of vascular and endocrine physiology taught in lectures. In addition, the multimedia material provides asynchronous teaching, as it can be consulted at any time.

Multimedia, integrative physiology, asynchronous teaching

P1-06

\section{INTEGRATING PHYSIOLOGY}

Maria Pilar Ribate, Laura Lomba, Estefanía Zuriaga, Beatriz Giner, Cristina B García

Universidad San Jorge, Villanueva de Gállego (Zaragoza), Spain

An innovative teaching activity has been designed to solve problems of social adaptation and to increase communication skills of people with an intellectual and development disability (IDD). ServiceLearning methodology has been applied on Physiology subject in $2^{\text {nd }}$ year of Pharmacy Degree training. It is an educational tool that combines learning of students through service in the community. People with IDD take advantage of the Physiological concepts provided by the university students. Furthermore, they would likely improve their communication abilities with health professionals regarding a better knowledge of body structures and functions.

On the other hand, our students are challenged to use their knowledges acquired in the subject to develop adapted activities to this community. To achieve this goal they have to change their role to teach pain, special senses (taste, smell and touch), bones and muscles. They have to design and carry out a lecture for people with IDD. At the end of this activity it is expected an increase in student's learning and tolerance. Satisfactory results with both, students and people with IDD, have been reached. This experience has been positive for the learning process in Physiology of our students that have been highly motivated as well as implicated teachers.

This type of innovative methodologies can be applied in other different communities such as immigrants, infants or elderly people. Finally, we can conclude that Integrating Physiology has been fully successfully: all the proposed objectives have been achieved, academic and social values.

Service-Learning, people with intellectual and development disabilities, role play.

P1-07

TEACHING PATTERNS IN BREATHING DISORDERS. BOARDING OF ASTHMA AS AN OBSTRUCTIVE RESPIRATORY PATTERN AND EVALUATION OF CRISIS BY CLINICAL PHYSIOLOGY STUDENTS ON A SIMULATIONBASED LEARNING
Gonzalo Tena ${ }^{(1)}$, Isabel Gutiérrez ${ }^{(1)}$, Jose Miguel Torrejón (1), Ángel Aumesquet (2), Ángela Navarro (1), Francisco Puerta (1), Charles Eric Juvin (1), Guillermo Álvarez De Toledo (3)

(1) Facultad de Medicina, Universidad de Sevilla, Sevilla, Spain; ${ }^{(2)}$ Facultad de Medicina, Universidad de Cádiz, Cádiz, Spain; (3) Departamento de Fisiología Médica y Biofísica, Universidad de Sevilla, Sevilla, Spain

Introduction: Medical simulation is been increasingly used as teaching tool not only for clinical training but for its capacity to reproduce faithfully the time course of physiological processes. For medical students, simulation sets the bases for integration of physiology into the clinical practice, for students of different disciplines, medical simulation can provide a realistic scenario of the complex interaction between physiological variables and the consequences of disease. The role of simulation is such that it is considered a valuable method for assessing the ability of students for before graduation by the ECOE test ("Objective Structured Clinical Evaluation"), which has been approach to the American model of vocational training.

Objective: To develop scenarios in SimMan to reproduce Asthma conditions with the purpose of understanding the physiology of the respiratory tract. Students will have to check how increased resistance in the airways affects ventilation, blood gases, and cellular respiration.

Methods: The mannequin software allows us to modify many respiratory parameters $\left(\mathrm{SatO}_{2} ; \mathrm{CO}_{2} ; \mathrm{inO}_{2} ;\right.$ etCO awRR, etc.) to closely reproduce the existing breathing patterns during an asthmatic crisis. The physiological variables are displayed in a UCI monitor previously programmed with the information obtained in patients or simulated by physiological software such as Human. Since the patient's vital signs are scheduled as trends, rather than static parameters, students can observed a quasi real time course of the changes of physiological variables depending on the measures taken by the students, who are receiving constant feedback of information denoting the importance of their decisions.

Prior to the development stage, students are trained to know the basic operation of the mannequin and the material available (stethoscope, identification of auscultation focus, use of intravenous or rescue medication, intubation techniques, types of facial masks, methods of mechanical ventilation noninvasive, etc.), allowing a comprehensive approach.

Results: We have used the SimMan simulation Model (Laerdal Medical Inc.) to adapt the pathophysiology of obstructive respiratory pattern that develops complications secondary to asthma attacks, which is a serious situation that is not hard to happen and it requires immediate resolution to avoid permanent damage and to relieve patient's distress.

Conclusions: The scenario of asthma made in SimMan closely resembles the pathophysiology of this diseases and students better understand the interaction of respiratory parameters in a more comprehensive way. Also, students are introduced to a rationale on how treatment should be conducted in this situation.

Asthma, obstructive respiratory pattern, simulation, SimMan 
P1-08

STUDY OF THE EVOLUTION OF CHRONIC OBSTRUCTIVE PULMONARY DISEASE (EPOC): PATHOPHYSIOLOGYAND RESOLUTION OF CRISIS. A SIMULATION-BASED TEACHING MODEL FOR A CLINICAL APPROACH

Gonzalo Tena ${ }^{(1)}$, Isabel Gutiérrez ${ }^{(1)}$, Jose Miguel Torrejón ${ }^{(1)}$, Ángel Aumesquet ${ }^{(2)}$, Ángela Navarro ${ }^{(1)}$, Francisco Puerta ${ }^{(1)}$, Charles Eric Juvin ${ }^{(1)}$, Guillermo Álvarez De Toledo ${ }^{(3)}$

(1) Facultad de Medicina, Universidad de Sevilla, Sevilla, Spain;

(2) Facultad de Medicina, Universidad de Cádiz, Cádiz, Spain;

(3) Departamento de Fisiología Médica y Biofísica, Universidad de Sevilla, Sevilla, Spain

Introduction: We have created a working group consisting of medical students, residents and university professors, to create clinical scenarios reproducing EPOC in a simulation mannequin. Objectives: Study and clinical practice of EPOC from an integrated comprehensive approach, giving emphasis to physiological concept in respiratory Physiology

Methods: We rely on the knowledge of students on respiratory physiology to develop four scenarios with different evolutions of EPOC. We've taken real unidentified medical records, seeking to identify a common evolution of these patients and individualize their processes in order to develop the scenarios in SimMan.

Due to the limited knowledge of general pathology in second year medical students, we have reduced the information on complementary diagnostic methods and emphasized the evolution of physiological variables.

Results: students practice in team of up to three and are expected to fulfill the medical history with information of the current episode after an exacerbation of EPOC that involves hospital admission due to its severity (blood pressure, identification of respiratory pattern and auscultation with stethoscope, pulmonary saturation levels, degree of congestive heart failure). Similarly we expect the student to explain the state of these parameters from a pathophysiological perspective.

Upon completion of cases, it is proposed to Debrief them, allowing participation of whole class as a clinical session.

Conclusions: These scenarios may serve for training medical students in the diagnosis and therapeutic management of EPOC in a safe environment where they can consolidate their theoretical knowledge and apply them to acquire the necessary confidence to develop their clinical practice where the patient is the main beneficiary.

EPOC, Chronic obstructive respiratory syndrome, Simulation, SimMan

LEARNING PHYSIOLOGY WITH COMPUTATION MODELS: CARDIO-RESPIRATORY ADJUSTMENTS DURING EXERCISE

Asuncion Rocher, Lucia Nunez, Irene Carretero, Angela GomezNiño, F. Javier García-Sancho

Facultad de Medicina, Univ. Valladolid, Valladolid, Spain
This work offers the opportunity to clinch Cardiocirculatory and Respiratory Physiology knowledge by simulation experiences in Human Physiology. We use a Simulation Laboratory located at the Medical School of Valladolid, which is provided with a high fidelity simulator manikin (iSTAN model, Medical Simulator, Germany) and the associated software (MÜSE) to improve learning methodology for medical students. The manikin has breath sounds, heart tones and palpable pulses providing real-time information to students. The manikin also has a monitor that can display EKG, pulse oximeter, blood pressure, arterial and pulmonary wave forms, etc. The MÜSE software integrates a pathophysiological model that we utilize to learn physiology by computerized simulation. This software is versatile and very integrative, but it needs planned sessions and tuning simulated practical examples to acquire competences and skills in Physiology and to promote student's reflective thinking. In accordance with these aims, we have elaborated several practical examples of circulatory and respiratory physiology. Here we present a simulation of physical exercise, their parameters progress after different intensities (light, moderate and intense, $10 \mathrm{~min}$ ) and their physiological adaptations. We focus our analysis mostly on cardiovascular and respiratory parameters: evolution of the mean arterial blood pressure, systolic, diastolic and pulse pressure, heart rate, cardiac output, temperature, oxygen consumption, pulmonary ventilation in exercise, oxygen diffusing capacity and blood gases during the exercise, $\mathrm{SatO} 2$ and recovery of the muscle metabolic systems after exercise (oxygen debt). Simulating exercise episodes causing increasing oxygen consumptions ( 1 to $5 \mathrm{~L} / \mathrm{min}$ ) we obtained relationships between heart rate, blood pressures and respiratory rate, and we can observe how the circulatory and respiratory variables are modified. From the analysis of these variables, the student can generate an overall picture of how the organism responds to moderate or intense exercise. At the end of the simulation, the students are invited to think over on causes and mechanisms for the observed cardio-respiratory adjustments, implications of muscle metabolic systems in exercise and, finally, the effect of training on muscles and muscle performance.

We conclude that the use of manikin simulation and computerbased mathematical models of physiological systems is potentially useful for linking the theoretical fundamentals and the practical teaching of physiology. It reinforces self-learning because the performance includes complementary information about previously discussed topics. It also serves an auto-evaluation function, as it includes multiple-choice questions. Finally, it is safe for the patients as it does only involve virtual ones.

Supported by grant: UVa PID2015-164.

Physiology Learning, Computation Model, Exercise Simulation, Cardio-Respiratory Physiology.

P1-10

\section{PHYSIOLOGY AND BIOSTATISTICS: AN EXAMPLE OF COLLABORATIVE INTERDISCIPLINARITY}

Eduardo Piedrafita, Carlos Valero, Sina Guillaumin, Manuel Gómez-Barrera, Santiago Lamas, Maria Pilar Ribate

Universidad San Jorge, Villanueva de Gállego, Spain 
General Physiology and Biostatistics are taught simultaneously in this Physiotherapy Degree at Universidad San Jorge which allows both subjects to design an activity that can be developed and evaluated. This teaching innovation project attempts to introduce students to research methods in order to help them in their learning process, for applying it to their professional practice. With cooperative work, scientific method will be applied to study physiological variables (heart rate, temperature, etc.) in a particular population. Students will have to establish a work plan including: literary research, data collection questionnaire, informed consent, and statistical analysis. Students will then be able to reach final conclusions, where variables will be justified depending on different environmental factors: age, sex, physical activity level, etc.

Physiological structures and processes will be described in 'Introduction'. Data collection process and information analysis will be included in 'Material \& Methods' and 'Results', respectively. Comparison with current bibliography and justification will be shown in 'Discussion and Conclusions'. Each section will be evaluated with rubrics ('Introduction' and 'Discussion and Conclusions' by General Physiology; 'Material \& Methods' and 'Results' by Biostatistics).

By the end of the activity, students should have acquired the following skills: ability to include scientific research and practice based on evidence as Physiotherapy professional culture, continuously updating knowledge and skills. In the General Physiology activity, students will be able to describe healthy organism systems functions, as well as integration processes for homeostasis. In Biostatistics, a suitable inclusion of methodology, results and conclusions from their research in a scientific article is expected.

Once the literary research and data collection questionnaire designs are done students will be invited to participate in research aims formulation. Then after data collection, statistical analysis stage and results and conclusions explanation will be started.

When students participate in all research stages, we believe their motivation is much higher than when they just participate in analysis stage with non-collected data. Besides, the students enjoy these theoretical concepts. Students could also gain a beneficial ethical perspective by dealing with the problems related to a deficient data collection, the advantages on processes accuracy of each research stage, and the need to be consistent with decisions adopted along the process.

Research stages, teaching innovation project, cooperative work.

P1-11

RESEARCH ABILITIES IN MEDICAL EDUCATION: AIMS, DESIGN AND OUTCOME EVALUATION OF A CORE COURSE IN "BIOMEDICAL RESEARCH AND NEW TECHNOLOGIES" AT UNIVERSITY OF VALLADOLID

D. Sánchez ${ }^{(1)}, M$. Hernández ${ }^{(1)}, N$. Fernández ${ }^{(1)}$, A Moreno $^{(1)}$, E. Arranz ${ }^{(1)}$, Jm Marugán $^{(1)}$, R. López-Izquierdo ${ }^{(1)}$, B. Rubia ${ }^{(2)}$, A. García-Vergara ${ }^{(2)}$, M. Maciel ${ }^{(2)}$, Md Ganfornina ${ }^{(1)}$

(1) Facultad Medicina; Univ. Valladolid, Valladolid, Spain;

(2) Facultad Educación; Univ. Valladolid, Valladolid, Spain
That Medicine is sustained on research-generated knowledge seems an obvious statement. However, the figure of "Physician+Researcher" so well accredited in other countries, is still far from being fully recognized and institutionally promoted in the Spanish Healthcare System.

The course "Biomedical Research and New Technologies" is part of the required courses taken during the second year of Medical School at University of Valladolid. Its global aim is to provide Medical Graduates with a solid education in biomedical research, focusing on practical research abilities to be applied, first during their undergraduate years (either as Research Interns, many times associated to Physiology Departments, or in their "End-of-Degree Project"), and later during their ongoing education as Medical Interns.

The course covers general concepts in research, its methods, and evidence-based medicine. It also covers practical abilities in data mining, critical analysis and communication of research results, critical appraisal, use of reference managers, image analysis, and quantitative data analysis.

In addition to regular evaluations of students learning process, our Teaching Team is carrying out an "Innovation in Teaching Research Project" to assess the long term impact of this course in Medical graduates. We designed a series of web-based screening tools to evaluate (i) the appreciation of research by students, (ii) their of use of research-related tools, and (iii) their competence in research-related tasks. These three domains are explored before they take the course, during their $6^{\text {th }}$ year of Medical School, and during the $1^{\text {st }}$ year of Medical Internship.

We are collecting a longitudinal series (2015-2018), covering graduates from the previous academic program, not including formal education on research, and graduates from the new system. Our exploration of Medical Interns has started in the two Valladolid University Hospitals, but aims at exploring other Universities to compare the outcome of diverse academic programs.

The results obtained during the first 2 years of our longitudinal analysis depict a very interesting scenario. Students entering their second year of Medicine show quite a high interest in research, while those graduating from the previous academic program reveal important deficiencies, both conceptual and ability-related, revealing needs that were not covered in their education.

We can conclude that the effort to introduce courses of this type, where formal education on research is undertaken, is worthwhile and can help significantly to the generation of Medical Doctors with a solid research education, with the foreseen beneficial consequences for the quality and excellence of our Healthcare System.

Biomedical research, education outcome evaluation, training in research abilities

$\mathrm{P} 1-12$

DIFFERENCES IN THE RESPONSE OF MALE AND FEMALE RATS TO MATERNAL UNDERNUTRITION DURING GESTATION

Cynthia Guadalupe Reyes Hernández (1), David Ramiro Cortijo ${ }^{(1)}$, Pilar Rodríguez Rodríguez ${ }^{(1)}$, Perla Yareli Gutiérrez Arzapalo (1), María Del Carmen González García ${ }^{(1)}$, Ángel Luis López De Pablo ${ }^{(1)}$, Silvia Magdalena Arribas Rodríguez ${ }^{(1)}$, Rodrigo Enrique Elizondo Omaña ${ }^{(2)}$, Santos Guzmán López ${ }^{(2)}$ 
(1) Universidad Autónoma de Madrid, Madrid, Spain;

(2) Universidad Autónoma de Nuevo León, Monterrey, Mexico

Introduction: Intrauterine growth restriction (IUGR) and low birth weight have been associated with the development of cardiovascular diseases (CVD) and some of its risk factors in adulthood, particularly hypertension (HTA). There are differences in the presentation of CVD between men and women, men present higher susceptibility to stress during fetal life. Gender differences in fetal abnormalities caused by fetal stress, have been attributed to sex hormones. The placenta has the same sex as the individual, the presence of differences in the ratio placenta: fetus could also help to explain the sexual dimorphism observed in the CVD in adulthood.

Aim: Was to study in an IUGR rat model, the maternal and fetoplacental alterations at the end of gestation, in order to determine whether there is sexual dimorphism.

Material and Methods: A model of IUGR induced by nutritional restriction in pregnant rats was utilized, in this model we restricted the diet to $50 \%$ of control intake during the second half of gestation, returning to ad libitum diet during lactation. At day 20 of gestation, we studied gender differences of the alterations observed in some maternal and fetal parameters.

Results: Pregnant rats subjected to nutritional restriction (R) had lower weight and a decrease in the concentration of total plasma proteins without altering blood glucose levels. R females had a smaller body size (weight and length) than males. The ratio placental weight: fetal weight was higher in $\mathrm{R}$ males than controls. The cord length was smaller in $\mathrm{R}$ males and females compared to their controls. The relative weight of heart and kidney was lower in $\mathrm{R}$ males, whereas in $\mathrm{R}$ females lower weight was only observed in the kidney but not in the heart compared to their respective controls.

Conclusions: Gender differences observed during the intrauterine stage could help to explain the subsequent sexual dimorphism observed in the development of CVD in adulthood; particularly the development of hypertension in men.

Acknowledgements. MINECO/FEDER (FEM2012-37634-C030 y FEM2015-63631-R).

Maternofetal alterations, intrauterine growth restriction (IUGR), sexual dimorphism,fetal programming,gestational undernutrition

P1-13

EARLY REGRESSION OF LEFT VENTRICULAR HYPERTROPHY WITH DRONEDARONE IN HYPERTENSIVE RATS

Begoña Quintana-Villamandos ${ }^{(1)}$, Jose Juan Gómez De Diego ${ }^{(2)}$, M Jesús Delgado-Martos ${ }^{(3)}$, M Carmen Fernández-Criado (4), David Muñoz ${ }^{(4)}$, Emilio Delgado-Baeza ${ }^{(3)}$

(1) Departamento Anestesiología Hospital General Universitario Gregorio Marañón, Madrid, Spain; ${ }^{(2)}$ Departamento Cardiología Hospital Clínico San Carlos, Madrid, Spain; ${ }^{(3)}$ Laboratorio Biología Molecular Unidad de Medicina y Cirugía Experimental Hospital General Universitario Gregorio Marañón, Madrid, Spain; ${ }^{(4)}$ Facultad de Medicina UAM, Madrid, Spain
Background: Regression of left ventricular mass with antihypertensive therapy (angiotensin-converting enzyme inhibitor, angiotensin receptor blocker, beta-adrenergic blocker, calcium channel blocker and diuretic) have been described in the literature after chronic treatments. However, early regression of left ventricular hypertrophy has not been reported following short-term administration of dronedarone. We tested the hypothesis that short-term administration of a new antiarrhythmic agent, dronedarone, produces early regression left ventricular hypertrophy in the SHR (spontaneously hypertensive rat) model of stable compensated ventricular hypertrophy.

Methods: We examined the effect of dronedarone on left ventricular hypertrophy and cardiac function in 11-month-old male SHRD. Age-matched and sex-matched SHR and Wistar Kyoto rats (WKY) were use as controls. After 15 days of treatment, left ventricular morphology and function were assessed from Mmode echocardiograms [left ventricular mass (LVM), ejection fraction $(\% \mathrm{EF})$ and transmitral Doppler [early-to-atrial filling velocity ratio (E/A), E-wave deceleration time (Edec time)]. The parameters were compared single-factor (rat) analysis of variance. A post hoc Bonferroni's correction was applied.

Results: Systolic blood pressure (SBP) was higher in untreated SHR than in the WKY controls. Dronedarone lowered SBP in treated SHR-D with respect to untreated SHR. LVM was significantly increased in untreated SHR when compared with the agematched WKY group. LVM was significantly attenuated in SHR$\mathrm{D}$ with drondarone with respect to untreated SHR. There were no significant changes in \%EF, E/A ratio nor in Edec time between WKY, SHR and SHR-D.

Conclusion: Dronedarone produces early regression left ventricular hypertrophy in the SHR model of stable compensated ventricular hypertrophy.

Acknowledgements: This study was supported by a grant from the Spanish Health Ministry (Fondo de Investigaciones Sanitarias) under contract FIS 13/01261 and Fondos Feder.

Dronedarone, hypertension, left ventricular hypertrophy

P1-14

CENPS AND AU-CENPS DECREASE SENSITIVITY TO NORADRENALINE WITHOUT MODIFYING ENDOTHELIAL FUNCTION

Solanye Guerra-Ojeda ${ }^{(1)}$, Patricia Marchio ${ }^{(1)}$, Marc Gimeno ${ }^{(1)}$, Victor M Victor ${ }^{(2)}$, Susana Rovira-Llopis ${ }^{(2)}$, Jose Raul Herance (3) Constanza Aldasoro ${ }^{(1)}$, Adrian Jordá (1), Alfonso Valverde ${ }^{(4)}$, Martin Aldasoro ${ }^{(1)}$, Soraya Lilian Vallés ${ }^{(1)}$, Jose María Vila ${ }^{(1)}$, María Dolores Mauricio (1)

(1) Departamento de Fisiología, Faculty of Medicine, Universitat de Valencia, Valencia, Spain; ${ }^{(2)}$ Service of Endocrinology, University Hospital Doctor Peset, Foundation for the Promotion of Health and Biomedical Research in the Valencian Region (FISABIO), Valencia, Spain; (3) 3 Vall d'Hebron Research Institute. Medical Molecular Imaging Group, Barcelona, Spain; ${ }^{(4)}$ Departamento de Anatomía y Embriología, Faculty of Medicine, Universitat de Valencia,, Valencia, Spain 
Introduction: Cerium nanoparticles (CeNPs) are used in some biomedical applications due to their size $(\leq 100 \mathrm{~nm}$ in at least one dimension) that creates a large surface area for the attachment of several drugs. CeNPs are also free radical scavengers; moreover, gold nanoparticles supported on CeNPs (Au-CeNPs) have been considered powerful agents against intracellular oxidative stress. Despite their growing pharmaceutical relevance, their effects on cardiovascular system remain unexplored.

Purpose: Fully understanding of how nanoparticles modulate the vascular response is critical for their development as potential therapeutic agents. Therefore, the purpose of this study was to test the vascular effects of CeNPs and Au-CeNPs in response to contractile and vasodilator agonists.

Methods: Rings of carotid artery (3 $\mathrm{mm}$ long) from seven male Sprague-Dawley rats were suspended in organ baths for isometric recording of tension. We studied the responses to acetylcholine $\left(10^{-9}-10^{-5} \mathrm{M}\right)$, and noradrenaline $\left(10^{-9}-3 \times 10^{-5} \mathrm{M}\right)$ in the absence and in the presence of CeNPs $(50$ and $100 \mathrm{mg} / \mathrm{ml})$ or AuCeNPs (50 and $100 \mathrm{mg} / \mathrm{ml})$.

Results: Acetylcholine $\left(10^{-9}-3 \times 10^{-6} \mathrm{M}\right)$ produced concentrationdependent relaxation in segments from rat carotid artery precontracted with noradrenaline. The Emax was similar in the absence and in the presence of CeNPs and Au-CeNPs. The incubation with CeNPs $(50 \mathrm{mg} / \mathrm{ml})$ did not modify noradrenaline curve, while CeNPs $(100 \mathrm{mg} / \mathrm{ml})$ significantly shifted the noradrenaline curve to the right in a parallel manner without changes in Emax. However, both tested concentrations of Au-CeNPs, 50 and $100 \mathrm{mg} / \mathrm{ml}$, diminished the sensitivity to noradrenaline.

Conclusions: Our results provide evidence that both, CeNPs $(100 \mathrm{mg} / \mathrm{ml})$ and Au-CeNPs $(50$ and $100 \mathrm{mg} / \mathrm{ml})$, alter the response to noradrenaline without affecting endotheliumdependent relaxation induced by acetylcholine. Au-CeNPs have more effect than CeNPs in decreasing sensitivity to noradrenaline. This finding demonstrated that nanoparticles could have beneficial effects in the treatment of diseases associated with elevated plasma levels of noradrenaline such as essential hypertension.

Cerium nanoparticles, free radical scavengers, vascular reactivity

$$
\text { P1-15 }
$$

\section{VASCULAR ADRENERGIC EFFECTS INDUCED BY RANOLAZINE}

Constanza Aldasoro ${ }^{(1)}$, Sol Guerra-Ojeda ${ }^{(2)}$, Patricia Marchio ${ }^{(2)}$, Maria Dolores Mauricio ${ }^{(2)}$, Marc Gimeno-Raga ${ }^{(2)}$, Adrián Jordá (2) Mariza Colque-Cano ${ }^{(2)}$, Alicia Bertomeu ${ }^{(2)}$, Martín Aldasoro ${ }^{(2)}$, Jose María Vila ${ }^{(2)}$, Soraya Lilian Vallés ${ }^{(2)}$

(1) Hospital General de Castellón, Castellón, Spain; (2) Departamento de Fisiología, Universidad de Valencia, Valencia, Spain

Introduction: Ranolazine ( $\mathrm{Rn})$ is used as an antianginal drug to treat patients with chronic cardiac conditions and may improve coronary blood flow by reducing compression effects of ischemic contracture, and by improving endothelial function. In addition to its antianginal effects, Rn acts as an anti-inflammatory and antioxidant agent by reducing IL- $\beta$ and TNF- $\alpha$ plasma levels, and promoting antioxidant proteins $\mathrm{Cu} / \mathrm{Zn}-\mathrm{SOD}$ and $\mathrm{Mn}-\mathrm{SOD}$ activity. In this study we investigate the vascular adrenergic effects of $\mathrm{Rn}$ on isolated rat aorta.

Methods: Rat aorta segments (3 mm long) were mounted for isometric tension recording in organ baths containing KrebsHenseleit solution. Electrical field stimulation (EFS) (2, 4 and $8 \mathrm{~Hz}, 20 \mathrm{~V}, 0.25 \mathrm{~ms}$ duration for $30 \mathrm{~s}$ ) was provided by a Grass S88 stimulator via two platinum electrodes on each side and parallel to vascular segment. The experiments were carried out in absence or presence of different Rn concentrations $\left(10^{-7}-10^{-5} \mathrm{M}\right)$. Concentration-response curves for noradrenaline were determined in the absence and presence of $\mathrm{Rn}\left(10^{-7}-10^{-5} \mathrm{M}\right)$. from separate artery preparations and concentration-response curves for $\mathrm{Rn}\left(10^{-7}-10^{-5} \mathrm{M}\right)$ were obtained in a cumulative manner using endothelin-1, noradrenaline, thromboxane $\mathrm{A}_{2}$ and vasopressin as constrictor agents. Western-blot assay was used to determine expression of adrenergic vascular receptors $\left(\alpha_{1}, \alpha_{2}\right.$ and $\left.\beta_{2}\right)$ in absence or presence of $\mathrm{Rn}\left(10^{-7}-10^{-5} \mathrm{M}\right)$.

Results: Contractile responses to EFS were abolished by tetrodotoxin, guanethidine and prazosin, indicating that this effect is due to the action of noradrenaline on $\alpha_{1}$ receptor. $\mathrm{Rn}$ diminished $(p<0.05)$ neurogenic contractions induced by EFS in aortic rings and this effect is not dependent on nitric oxide or prostanoid release. Rn displaced the control curve for noradrenaline to the right in a parallel manner without differences in the maximal tension and produced concentrationdependent relaxation in rings contracted previously with noradrenaline (Emax $86 \pm 6 \% ; p<0.05)$ but not in those with endothelin-1, thromboxane A2 or vasopressin. Neither LNAME $\left(10^{-4} \mathrm{M}\right)$, an inhibitor of nitric oxide synthase, nor indomethacin $\left(10^{-5} \mathrm{M}\right)$, modified the relaxation induced by Rn. Furthermore, Rn decreased $\alpha_{1}$ and $\alpha_{2}$ and increased $\beta_{2}$ adrenergic receptor expression in aortic segments compared with control rat.

Conclusions: $\mathrm{Rn}$ diminished the contractile response induced by adrenergic stimulation, suggesting an $\alpha_{1}$ adrenergic antagonist effect. Relaxant effects of Rn on rat aortic vessels don't depend on the endothelium-derived factors. Rn diminished $\alpha_{1}$ and $\alpha_{2}$ and increased $\beta_{2}$ expression. Given that $\alpha_{1}$ and $\alpha_{2}$ mediate vasoconstriction and $\beta_{2}$ vasodilatation, our assembled results could explain the Rn-induced vascular effects.

Ranolazina, Vascular, Diabetes, Adrenérgico,

\section{P1-16}

DEVELOPMENT AND CHARACTERIZATION OF AN EXPERIMENTAL MODEL OF DIET-INDUCED METABOLIC SYNDROME IN RABBIT

Óscar Julián Arias-Mutis ${ }^{(1)}$, Patricia Genovés ${ }^{(1)}$, Ana Díaz ${ }^{(2)}$, Mariano Muñoz ${ }^{(2)}$, Luis Such-Miquel ${ }^{(2)}$, Irene Del Canto ${ }^{(1)}$, Carlos Soler ${ }^{(2)}$, Antonio Alberola ${ }^{(2)}$, Manuel Zarzoso ${ }^{(2)}$

(1) Research Foundation Clinic Hospital of Valencia (INCLIVA), Valencia, Spain; ${ }^{(2)}$ Universitat de València, Valencia, Spain 
Introduction: Metabolic syndrome $(\mathrm{MeS})$ has become one of the main concerns for public health because of its link to cardiovascular disease and cost in the healthcare system. Murine animal models have been used to study the impact of $\mathrm{MeS}$ on the cardiovascular system but they have serious limitations for studying cardiac electrophysiology. In contrast, the rabbit shows cardiac electrophysiological characteristics similar to human, and its maintenance is not as costly as larger animal models. Our aim was to develop and characterize a diet-induced experimental model of MeS that allows the study of cardiac electrical remodeling and arrhythmogenesis.

Methods: 25 male NZW rabbits were assigned to a control $(n=12)$ or MeS group $(n=13)$, fed during 28 weeks with highfat (10\% hydrogenated coconut oil and $5 \%$ lard), high-sucrose ( $15 \%$ dissolved in water) diet. We measured weight, morphological characteristics (abdominal contour, height, length, BMI), blood pressure and glycemia (fasting glucose and during a glucose tolerance test, GTT), before diet administration and at weeks 14 and 28. A mixed model ANOVA and unpaired $t$-test were used for statistical analysis $(p<0.05)$.

Results: Animals in MeS group showed an 18 and $24 \%$ increase in weight at weeks 14 and 28, respectively. Even though we did not find differences in morphological parameters such as length and height, the abdominal contour and BMI increased in the experimental group at weeks 14 and 28. Systolic, diastolic and mean arterial pressure (MAP) increased in MeS group at week 14 and 28 with respect to control group (i.e. MAP: $78 \pm 8$ vs $82 \pm 8 \mathrm{mmHg}$ at week 14 , $80 \pm 3$ vs $93 \pm 5$ at week $28 ; p<0.05)$. Fasting blood glucose was higher in the MeS group at week $14(115 \pm 10$ vs 102 $\pm 8 \mathrm{mg} / \mathrm{dL} ; p<0.05)$ and $28(116 \pm 10$ vs $100 \pm 9 \mathrm{mg} / \mathrm{dL}$; $p<0.05)$. GTT showed differences between the two groups and the area under the curve increased in the MeS group at weeks $14(1624 \pm 185$ vs $2062 \pm 330$ a.u.; $p<0.05)$ and 28 $(1570 \pm 99$ vs $2020 \pm 381$ a.u.; $p<0.05)$.

Conclusion: Administration of high-fat and high-sucrose diet during 28 weeks induced obesity, glucose intolerance and elevation of systolic, diastolic and mean blood pressure, thus reproducing some of the clinical manifestations of the metabolic syndrome in humans. The experimental model could be used in future studies to investigate the cardiac electrical remodeling produced by this pathological condition.

Supported by grants UV-INV-PRECOMP14-206372, GV2015062 and RD12/0042/0048 (ISCIII-FEDER funds).

Metabolic syndrome, experimental model, high-fat and highsucrose diet, cardiovascular system

\section{P1-17}

CONTRACTILE PROPERTIES OF RAT BASILAR ARTERY IN A MODEL OF SUBARACHNOID HEMORRHAGE: ROLE OF L-TYPE $\mathrm{CA}^{2+}$ CHANNELS, SARCOPLASMIC RETICULUM AND RHO KINASE

María Del Carmen González-Montelongo ${ }^{(1)}$, Juan José EgeaGuerrero ${ }^{(2)}$, Francisco Murillo-Cabezas (2), María Ángeles Muñoz-Sánchez ${ }^{(2)}$, Ángel Vilches-Arenas (3), Cristina PorrasGonzález $^{(1)}$, Antonio Castellano ${ }^{(1)}$, Juan Ureña ${ }^{(1)}$
(1) Instituto de Biomedicina de Sevilla (IBiS), Hospital Universitario Virgen del Rocío/CSIC/Universidad de Sevilla, Departamento de Fisiología Médica y Biofísica, Avenida Manuel Siurot s/n, 41013 Sevilla, Spain, Sevilla, Spain; ${ }^{(2)}$ Instituto de Biomedicina de Sevilla (IBiS), Hospital Universitario Virgen del Rocío/CSIC/Universidad de Sevilla, Unidad de Neurocríticos, Avenida Manuel Siurot s/n, 41013 Sevilla, Spain, Sevilla, Spain; (3) Instituto de Biomedicina de Sevilla (IBiS), Hospital Universitario Virgen del Rocío/CSIC/Universidad de Sevilla, Departamento de Medicina Preventiva y Salud Pública, Avenida Manuel Siurot s/n, 41013 Sevilla, Spain, Sevilla, Spain

Objective: We have previously described thatL-type $\mathrm{Ca}^{2+}$ channels (LTCCs) induce RhoA/Rho-associated Kinase (ROCK) activation through a metabotropic pathway (LTCCs/G protein/phospholipase C (PLC)/sarcoplasmic reticulum (SR) $\mathrm{Ca}^{2+}$ release). We have investigated the role of these routein contractile properties of arterial rings in subarachnoid hemorrhage (SAH).

Methodology: All experiments were conducted in accordance with the Spanish and European Union legislation on protection of experimental animals. The study based, on a new percutaneous subarachnoid hemorrhage model, was conducted using male Wistar rats divided in three experimental groups: control, sham and SAH. Basilar arteries were quickly removed from anesthetized animals, cleaned of the adjacent connective tissue, cut in rings and mounted on a small-vessel myograph. Arterial rings reactivity was studied on days 1 to 5 after surgery in basal conditions and in response to high potassium solutions. Experiments were performed at $30{ }^{\circ} \mathrm{C}$. The $70 \mathrm{~K}$ and $30 \mathrm{~K}$ solutions were obtained by replacing $70 \mathrm{mM}$ or $30 \mathrm{mM}$ of $\mathrm{NaCl}$ with $\mathrm{KCl}$. Before experiments, all segments were normalized to an optimal tension. Drugs were directly added to the chamber while vessel force was monitored. Data are expressed as mean $\pm \mathrm{SEM}$; $\mathrm{n}$ represents the number of arterial rings.

Results: In unstimulated arteries a significant increase of basal tone, measured $60 \mathrm{~min}$ after normalization, was observed on day 5 after SAH. This phenomenon was suppressed with low concentrations of nifedipine and fasudil, LTCCs and ROCK inhibitors, respectively. Depolarization with high $\mathrm{K}$ triggered an isometric contraction that showed an initial fast, transient (phasic), component followed by a slowly rising (tonic) component. Interestingly, there were no significant differences in the phasic component between sham and SAH groups, whereas a significant increase in the sustained contraction was observed on day 5 after SAH. Tonic component was sensitive to fasudil, and selectively reduced by nifedipine, U73122 and ciclopiazonic acid, LTCCs, PLC and SR-ATPase inhibitors, respectively.

Conclusions: Our data suggest that the metabotropic function of LTCCs is potentiated in arterial rings from pathological arteries. Our results could provide a new strategy to optimize the pharmacological treatment of arterial vasospasm present in aneurismal subarachnoid hemorrhage.

Keywords: Subarachnoid hemorrhage; Vasospasm; Basilar arterial rings; L-type $\mathrm{Ca}^{2+}$ channels; RhoA/Rho kinase; Sarcoplasmic reticulum.

Subarachnoid hemorrhage; Vasospasm; Basilar arterial rings; L-type $\mathrm{Ca}^{2+}$ channels; RhoA/Rho kinase; Sarcoplasmic reticulum. 
P1-18

MODIFICATIONS OF THE SPECTRAL CHARACTERISTICS OF VENTRICULAR FIBRILLATION PRODUCED IN AN EXPERIMENTAL RABBIT MODEL OF METABOLIC SYNDROME

Óscar Julián Arias-Mutis ${ }^{(1)}$, Conrado Calvo ${ }^{(2)}$, Luis Such-Miquel (3), Álvaro Tormos ${ }^{(2)}$, Antonio Guill ${ }^{(2)}$, José Millet ${ }^{(2)}$, Francisco Javier Chorro ${ }^{(1)}$, Antonio Alberola (3), Luis Such (3), Manuel Zarzoso ${ }^{(3)}$

(1) Research Foundation Clinic Hospital of Valencia (INCLIVA), Valencia, Spain; ${ }^{(2)}$ ITACA. Universitat Politècnica de València, Valencia, Spain; (3) Universitat de València, Valencia, Spain

Introduction: Metabolic syndrome $(\mathrm{MeS})$ has become a major public health concern due to its increased prevalence, which portends a greater risk of cardiovascular disease such as heart failure and sudden cardiac death (SCD). Indeed, it has been shown that obese and diabetic patients have a higher incidence of arrhythmias that are non-related to ischemic events, suggesting a pro-arrhythmic structural substrate and/or electrical remodeling, but the underlying mechanisms are not completely understood. Our aim was to investigate the cardiac electrical remodeling produced in an experimental model of diet-induced MeS by means of the study of the spectral characteristics of ventricular fibrillation (VF).

Methods: Twenty-five NZW rabbits were randomly assigned to a control $(n=12)$ or a MeS group $(n=13)$, fed during 28 weeks with high-fat (10\% hydrogenated coconut oil and $5 \%$ lard), high-sucrose (15\% dissolved in water) diet. After 28 weeks, their hearts were isolated and perfused in a Langendorff system and epicardial optical mapping was performed using two synchronized EMCCD cameras focused on the left (LV) and right (RV) ventricles. The field of view was $2.5 \times 2.5 \mathrm{~cm}(128 \times 128$ pixels $)$ and the sampling frequency 330 frames/second. We used the electromechanical uncoupler blebbistatin $(7.5 \mu \mathrm{M})$ and the potentiometric dye di-4-ANBDQPQ. VF was induced by pacing at increasing frequency and, once triggered, $10 \mathrm{~s}$ recordings were made each minute, during $5 \mathrm{~min}$ (without interrupting perfusion). We analyzed, in a region of interest of $80 \times 80$ pixels, the dominant frequency (DF) of VF and other parameters related to electrophysiological heterogeneity such as coefficient of variation (CV) of DF, regularity index (RI) and $\mathrm{CV}$ of RI. A mixed model ANOVA was used for statistical analysis $(p<0.05)$.

Results: No differences were found in DF of VF between groups or when comparisons were made within groups between RV and LV. Regarding electrophysiological heterogeneity, we observed an increase in $\mathrm{CV}$ of $\mathrm{DF}$ in the $\mathrm{LV}$ of both experimental groups $(p<0.01)$. Likewise, when comparisons were made within groups, RI and its $\mathrm{CV}$ increased in the LV of MeS and control groups $(p<0.05)$. On the other hand, no differences were found in electrophysiological heterogeneity between control and MeS groups.

Conclusion: High-fat and high-sucrose diet administration during 28 weeks did not modify the spectral characteristics of VF. Simultaneous optical mapping of RV and LV allowed us to find, however, differences in the electrophysiological heterogeneity between both ventricles during VF, being the heterogeneity higher in the LV.

Supported by grants UV-INV-PRECOMP14-206372, GV2015062 and RD12/0042/0048.

Metabolic syndrome, optical mapping, ventricular fibrillation, electrophysiological heterogeneity

P1-19

OVEREXPRESSION OF ENDOGLIN CAUSES VESSEL WALL DEFECTS IN A MURINE TUMOR ANGIOGENESIS MODEL

Claudia Ollauri Ibáñez, Elena Núñez Gómez, Lucía Pérez Roque, Laura Ruiz Remolina, Cristina Egido Turrión, José Miguel López Novoa, Alicia Rodríguez Barbero, Miguel Pericacho Bustos

Facultad de Medicina - Universidad de Salamanca, Salamanca, Spain

Solid tumors are composed by cancerous cells that grow in a rapid and uncontrolled way. As a consequence, great oxygen and nutrient requirements are generated and angiogenesis, the creation of new blood vessels that feed the tumor, becomes necessary. In clinical practice, one of the most used methods to study solid tumor microvessel density is endoglin (ENG) immunohistochemistry, correlating high levels of this protein with poor prognosis. Endoglin is a membrane glycoprotein whose expression is upregulated in tissues undergoing active angiogenesis. Endoglin deficiency causes Hereditary Hemorrhagic Telangiectasia (HHT), a vascular disease that courses with severe epistaxis and arteriovenous malformations. Moreover, $\mathrm{Eng}^{-/}$mice die in the embryonic development with severe problems in vasculogenesis. Furthermore, tumor xenografts developed in $\mathrm{Eng}^{+/-}$mice are smaller and less vascularized than those grown in WT mice. For this reason, it has been traditionally hypothesized that poor prognosis associated to high levels of endoglin is a consequence of an enhanced angiogenesis and thus, a larger tumor size. In this study, we test this hypothesis through the generation of tumor xenografts in mice that ubiquitously overexpress human membrane endoglin $\left(E N G^{+}\right)$. For this purpose, we injected a mixture of Lewis Lung Carcinoma (3LL) cells and Matrigel ${ }^{\circledR}$ in the flank of $E N G^{+}$and wild type (WT) mice. Contrary to expectations, tumors developed in $E N G^{+}$mice were not bigger than those grown in WT mice. No differences were found in the expression of endothelial markers nor in the number of vessels. WT tumor vessels were tortuous and unstable, as already described, but those present in $\mathrm{ENG}^{+}$tumors were even worse, leading to hemorrhages and big extravascular areas occupied by erythrocytes. This evidence explains why $\mathrm{ENG}^{+}$tumors also showed higher hemoglobin content and less concentration of DNA than WT tumors. We also found lower expression of mural cell markers in tumors grown in $E N G^{+}$ mice. In conclusion, overexpression of membrane endoglin leads to vessel wall defects and increased permeability that allow erythrocytes extravasation but also may permit tumor 
cell passage to the circulation. In view of these results, we can now reformulate our hypothesis and put forward that the worse prognosis related to increased endoglin levels would not be caused by the increased tumor growth but to an increased risk of metastasis.

Endoglin, angiogenesis, cancer

P1-20

INFLUENCE OF HYPERTENSION ON MESENTERIC SYMPATHETIC NEUROTRANSMISSION AND ITS MODULATION BY 5-HYDROXYTRYPTAMINE IN RATS

José Ángel García-Pedraza ${ }^{(1)}$, Enrique García-Nieto ${ }^{(2)}$, Laura Centeno ${ }^{(2)}$, Miriam Gómez-Roso ${ }^{(1)}$, Mónica García ${ }^{(1)}$, Asunción Morán ${ }^{(1)}$

(1) Departamento de Fisiología y Farmacología. Facultad de Farmacia. Universidad de Salamanca/Instituto de Investigación Biomédica de Salamanca (IBSAL), Salamanca., Salamanca, Spain; ${ }^{(2)}$ Departamento de Fisiología y Farmacología. Facultad de Farmacia. Universidad de Salamanca., Salamanca, Spain

The mesenteric vascular tone plays an important role in the regulation of the large splanchnic blood volume, highlighting the control by sympathetic neurotransmission. Some diseases can be associated with disturbances in this mesenteric innervation, as hypertension, leading to cardiovascular disorders. It has been reported that endogenous neuromodulators, as 5hydroxytryptamine (5-HT), can modify adrenergic activity, contributing to vascular homeostasis. Our work aimed to determine the influence of sympathetic neurotransmission on mesenteric vascular tone in normotensive and hypertensive rats, and investigate whether 5-HT modulates locally this noradrenergic innervation. Hypertension was induced by L-NAME (nitric oxide synthase inhibitor; $30 \mathrm{mg} / \mathrm{kg} /$ day in drinking water) for 21 days, monitoring body weight, systolic blood pressure and heart rate (HR). A normotensive group was run in parallel to serve as agematched controls. After 21 days, rats were anaesthetized (sodium pentobarbital; $60 \mathrm{mg} / \mathrm{kg}$, i.p.), and prepared for the in situ autoperfused rat mesentery, which allows continuous measurement of systemic blood pressure (SBP), HR and mesenteric perfusion pressure (MPP). Electrical stimulation of mesenteric sympathetic nerves and i.a. noradrenaline (NA) administration induced increases in MPP, without modifying SBP or HR. The electrical-induced increases in MPP were $8.4 \pm 0.8,21.6 \pm 2.1$ and $56.4 \pm 5.3 \mathrm{mmHg}$ for 2,4 and $8 \mathrm{~Hz}$, respectively, and NAevoked increases in MPP were $14.8 \pm 3.2,32.8 \pm 6.7$ and 78.2 $\pm 5.8 \mathrm{mmHg}$ for $0.1,0.3$ and $1.0 \mu \mathrm{g} / \mathrm{kg}$, respectively, in normotensive rats. However, in hypertensive rats the MPP increases were enhanced both by electrical stimulation $(12.3 \pm 1.6,37.5$ \pm 3.4 and $85.8 \pm 5.1 \mathrm{mmHg}$ for 2,4 and $8 \mathrm{~Hz}$, respectively) and by NA administration $(21.8 \pm 2.5,50.8 \pm 13.5$ and 110.3 $\pm 25.1 \mathrm{mmHg}$ for $0.1,0.3$ and $1.0 \mu \mathrm{g} / \mathrm{kg}$, respectively). All these mesenteric vasoconstrictor responses were abolished by pretreatment with prazosin $(250 \mu \mathrm{g} / \mathrm{kg}$; i.v.). Bolus of 5 -HT $(1.0,6.3$, 12.5 and $25 \mu \mathrm{g} / \mathrm{kg}$; i.a.) inhibited dose- and frequencydependently the vasopressor responses by mesenteric nerve electrical stimulation in both groups. However, $5-\mathrm{HT}(25 \mu \mathrm{g} / \mathrm{kg}$; i.a.) only reduced the NA-induced vasoconstrictions in normotensive rats. Our outcomes suggest that hypertension modifies the mesenteric innervation, enhancing mesenteric sympathetic activity. 5-HT reduces this mesenteric sympathetic neurotransmission in both groups: pre and/or postjunctionally in normotensive and exclusively prejunctionally in hypertensive animals. These results on mesenteric sympathetic innervation and its regulation by 5-HT could offer new fronts for knowledge of cardiovascular physiology and possible therapeutic management of hypertension.

5-hydroxytryptamine, Autoperfused rat mesentery, Hypertension, Sympathetic neurotransmission

P1-21

A MECHANISM FOR THE EFFECTIVENESS OF THE BIOREGULATORY MEDICAMENT ENGYSTOL BY STIMULATING THE CHEMOTAXIS OF NEUTROPHILS VIA TLR2

Leticia Martín-Cordero ${ }^{(1)}$, Juan Luis Quevedo ${ }^{(2)}$, María Dolores Hinchado ${ }^{(3)}$, Pedro Manonelles ${ }^{(2)}$, Eduardo Ortega ${ }^{(3)}$

(1) Dpto. Enfermería (Grupo Inmunofisiología). C.U.P. UEx, Plasencia, Spain; ${ }^{(2)}$ Universidad Católica San Antonio de Murcia, Murcia, Spain; ${ }^{(3)}$ Dpto. Fisiología (Grupo Inmunofisiología). Facultad Ciencias. UEx, Badajoz, Spain

The primary objective of most natural complementary therapies is to activate and regulate an organism's endogenous defence by strengthening the natural immune response. In this context, it is necessary to explore how the immune system is affected by multi-target combinations in order to demonstrate the scientific rationale underlying the benefits frequently reported in clinical practice. Engystol (Heel GmbH, BadenBaden, Germany) is reported as a multi-target bioregulatory medication (containing Vicetoxicum hirundinaria and sulphur) that has been used for more than 60 years. The prophylactic and complementary treatment benefits of Engystol against infections, and its safety, have been observed in clinical practice. Since peripheral leukocytes have been proposed as one of the best marker for evaluating complementary and alternative medicine, and that chemotaxis of neutrophils toward the site of infection is a good index for the activation status of phagocytic cells.

Objective: The present investigation was to evaluate the in vitro effect of Engystol on the chemotaxis of neutrophil. In addition, TLR2 has been demonstrated to be one of the most important TLRs on the surface of human neutrophils involved in activation of the innate response. Thus, it has been also investigated the role of TLR2 for mediating the stimulation of chemotaxis. Finally, this study also evaluated the potential chemoattractant effect of Engystol.

Methods: Chemotaxis of isolated neutrophils was evaluated in Boyden Chamber (with fMLP used as chemoattractant), both in the presence or absence of anti-TLR2 in order to evaluate the role of this receptor in the stimulation of neutrophils chemotaxis. The chemoattractant capacity of Engystol was also evaluated in 
Boyden Chambers, Engystol being used to induce chemotaxis of neutrophils.

Results: Engystol significantly stimulated the chemotaxis of neutrophils induced by fMLP (as a chemoattractant agent). Neutrophils pre-incubated with anti-TLR2 showed lower chemotaxis than those incubated only with Engystol and presented no significant differences with respect to the control values. Engystol is also chemoattractant for neutrophils, the observed migration was higher than that obtained with fMLP.

Conclusion: Engystol can contribute to the preventive and therapeutic actions against infections through the stimulation of chemotaxis of neutrophils, and TLR2 appears to be involved in this process. This study constitutes the first investigation involving an innate receptor in the stimulation of neutrophils chemotaxis by a bioregulatory medicament.

This investigation has been partially supported by Gobierno de Extremadura-FEDER (GR 15041) and an agreement UEX-“Laboratorios Heel España S.A.U” (211/14)

Engystol, Neutrophils, Chemotaxis, TLR2

$$
\text { P1-22 }
$$

\section{EFFECT OF HEAT SHOCK PROTEINS ON CELL VIABILITY AND CASPASES ACTIVATION IN HELA CELLS}

Ishak Suat, Roberto Pariente, Ignacio Bejarano, Ana Beatriz Rodríguez, Mustafa Naziroglu, Jose Antonio Llanos

University of Extremadura, Badajoz, Spain

In the present study, we investigated the effect of heat shock proteins (HSP) on apoptotic cell death on human epitheloid cervix carcinoma HeLa cell line.

Cells were heat shocked (HS) for $1 \mathrm{~h}$ at $42{ }^{\circ} \mathrm{C}$, followed by incubation at $37^{\circ} \mathrm{C}$ for $1,2,4,6$ and $24 \mathrm{~h}$. Cell viability was determined using the MTT assay and caspase $-3,-8$ and -9 enzymatic activities were determined by fluorimetric methods using the specific fluorigenic substrates. The chromatin condensation of apoptotic cells was revealed upon vital staining (10 $\mathrm{min}$ in the dark) with Hoechst 33342.

Our results show that the heat shock at $42{ }^{\circ} \mathrm{C}$ of HeLa cells produces a decrease in cell viability which was maintained for $24 \mathrm{~h}$. However, when HeLa cells were preincubated with $100 \mathrm{nM}$ geldanamycin (GA) (HSP90 inhibitor), $100 \mathrm{nM}$ 17-(allylamino)-17-demethoxygeldanamycin (17AAG) (HSP90 inhibitor) or $1 \mu \mathrm{M}$ pifithrin- $\mu$ (PFT- $\mu$ ) (HSP70 inhibitor) for $24 \mathrm{~h}$, cell viability was significantly $(p<0.05)$ lower than that induced by heat shock at all times tested. In addition, the heat shock of HeLa cells induced an increase in enzymatic activity of caspase-3, -8 and -9 , which were maintained for $24 \mathrm{~h}$. However, when the cells were preincubated in the presence of inhibitors of HSP (GA, 17AAG or PTF- $\mu$ ) activities of caspases were significantly $(p<0.05)$ increased. Finally, the inhibition of HSP90 by pretreatment the cells with GA or 17AAG or HSP 70 with PFT- $\mu$, enhanced the loss of HeLa adherence and the increased of nucleus with condensed chromatin. Such events are closely related to apoptotic processes.
Therefore these results support the protective role of HSP90 and HSP70 against the cell damage caused by heat shock.

Our results suggest that HSP evoked by heat shock have protective activities on HeLa cells, and are involved in cell survival. The inhibition of HSP with specific inhibitors induce cell cytotoxicity and activation of caspases.

Supported by Junta de Extremadura-Fondo Social Europeo de Desarrollo Regional (FEDER) grant IB13072 and GR15051.

HSP, caspases, cell viability

P1-23

GENE EXPRESSION AND ANTIOXIDANT SYSTEMS MODULATION IN A MURINE HIPPOCAMPAL-DERIVED CELL LINE SECONDARY TO SUB-TOXIC ETHANOL EXPOSURE

David Quinto-Alemany ${ }^{(1)}$, Verónica Casañas-Sánchez ${ }^{(2)}$, José A. Pérez ${ }^{(2)}$, Fátima Mesa-Herrera ${ }^{(1)}$, Carla Ferri ${ }^{(1)}$, Ana CanerinaAmaro ${ }^{(3)}$, Juan C. Sánchez-Hernández ${ }^{(4)}$, Raquel Marín ${ }^{(3)}$, Mario Díaz ${ }^{(1)}$

(1) Laboratory of Membrane Physiology and Biophysics, Department of Animal Biology, Universidad de La Laguna. Associate Research Unit ULL- CSIC "Membrane Physiology and Biophysics in Neurodegenerative and Cancer Diseases", La Laguna, Spain; ${ }^{(2)}$ Department of Genetics, University Institute of Tropical Diseases and Public Health, Universidad de La Laguna, La Laguna, Spain; ${ }^{(3)}$ Laboratory of Cellular Neurobiology, Department of Basic Medical Sciences, Universidad de La Laguna. Associate Research Unit ULL- CSIC "Membrane Physiology and Biophysics in Neurodegenerative and Cancer Diseases", La Laguna, Spain; ${ }^{(4)}$ Laboratory of Ecotoxicology, Faculty of Environmental Science and Biochemistry, Universidad de Castilla-La Mancha, Toledo, Spain

Introduction: Ethanol is known to cause severe systemic damage often explained as due to oxidative stress. Brain is particularly vulnerable to ethanol-induced reactive oxygen species (ROS) because the high amounts of lipids, and because nerve cell membranes contain high amounts of peroxidable fatty acids. Usually these effects of ethanol are associated to high and/or chronic exposure. However, several lines of evidence have shown that consumption of low doses of ethanol may provide neuroprotective effects against neurodegenerative diseases.

Objectives: The aim of the present study was to examine the possible mechanisms by which moderate ethanol concentrations might exert a neuroprotective effect on hippocampal cells through modification of cellular antioxidant capabilities.

Methods: The immortalized mouse hippocampal cell line, HT22, was cultured in standard Dulbecco's modified Eagle's medium (DMEM), replaced for the experiments with standard medium supplemented with ethanol in a $0.1 \%$ final concentration or vehicle phosphate buffered saline 
(PBS). Every $24 \mathrm{~h}$ of incubation the medium was replaced with fresh medium containing ethanol in PBS or PBS. Cell cultures were collected at $6,24,30$ and $48 \mathrm{~h}$, and immediately processed for either total RNA extraction, required for real-time reverse transcription quantitative PCR (RTqPCR) experiments, or preparation of total extracts required for determination of enzyme activities, and levels of glutathione and thiobarbituric reactive substances (TBARS).

Results: We have observed that $0.1 \%$ ethanol exposure to HT22 cells increases the transcriptional expression of different genes belonging to the classical, glutathione/glutaredoxin and thioredoxin/peroxiredoxin antioxidant systems, these including Sod1, Sod2, Gpx1, Gclc and Txnrd1. Paralleling these changes, enzyme activities of total superoxide dismutase (tSOD), catalase, total glutathione peroxidase (tGPx), glutathione- $S$-reductase (GSR) and total thioredoxin reductase (tTXNRD), were all increased, while the generation of TBARS, as indicators of lipid peroxidation, and glutathione levels remained unaltered. Ethanol exposure did not affect cell viability or cell growing as assessed by real-time cell culture monitoring, indicating that low ethanol doses are not deleterious for hippocampal cells, but rather prevented glutamate-induced excitotoxicity.

Conclusions We demonstrate for the first time, that sub-toxic ethanol exposure triggers the activation of cellular antioxidant systems and that its effects may be unravelled both at transcriptional and enzymatic levels. So, at low doses, ethanol exerts a role as an "Indirect Antioxidant", and that sub-toxic exposure may well be neuroprotective against oxidative insults, at least in hippocampal cells.

Acknowledgements: Supported by grants SAF2010-22114-C0201 and SAF2014-52582-R from MINECO (Spain).

Ethanol, antioxidant systems, hippocampal cells, HT22 cells

$\mathrm{P} 1-24$

LIPID RAFT ALTERATIONS IN HUMAN SHSY-5Y CELLS IN A MODEL OF PARKINSON'S DISEASE-LIKE NEUROTOXICITY

Ana Canerina-Amaro (1), Ricardo A. Puertas-Avendaño ${ }^{(1)}$, Miriam González-Gómez ${ }^{(1)}$, Ibrahim González-Marrero ${ }^{(1)}$, Carla Ferri ${ }^{(2)}$, Fátima Mesa-Herrera ${ }^{(2)}$, David Quinto-Alemany ${ }^{(2)}$, Mario Díaz ${ }^{(2)}$, Raquel Marín ${ }^{(1)}$

(1) Laboratory of Cellular Neurobiology, Department of Basic Medical Sciences, Universidad de La Laguna. Associate Research Unit ULL- CSIC "Membrane Physiology and Biophysics in Neurodegenerative and Cancer Diseases"., San Cristóbal de La Laguna, Spain; ${ }^{(2)}$ Laboratory of Membrane Physiology and Biophysics, Department of Animal Biology, Universidad de La Laguna. Associate Research Unit ULL- CSIC "Membrane Physiology and Biophysics in Neurodegenerative and Cancer Diseases". ., San Cristóbal de La Laguna, Spain

Previous work from our group has shown that structural lipids of Lipid Rafts microdomains are altered in cortical areas of Parkinson's disease (PD), even at early preclinical stages. In a murine model of PD treated with MPTP (1- methyl-4-phenyl1,2,3,6-tetrahydropyridine) as neurotoxic, we have previously found that similar alterations in the lipid composition of Lipid Rafts were associated with changes in the dynamic, aggregation and interaction of Lipid Raft resident proteins. Those alterations represent a potential trigger for aberrant signaling pathways, leading to cognitive decline that takes place during neuropathological progression.

In an attempt to further elucidate raft protein trafficking and intracellular signaling pathways involved in these findings, we have used here human neuroblastoma SHSY-5Y cells to induce PD-like neurotoxicity. Thus, SHSY- 5Y cells were treated with MPTP, and Lipid Rafts were isolated following Song et al., 1996. Lipid Rafts and Non-Raft membranes were analyzed by western blot and immunoprecipitation using alpha-synuclein specific antibodies. Ganglioside composition and cholesterol content was also determined. Remarkably, alpha-synuclein expression was increased in those cells treated with MPTP, as compared controls treated with vehicle. These results confirm our previous data in MPTP-treated mice, and indicate that SHSY-5Y cell line is a valid model for studying Lipid

Raft-mediated trafficking and signaling pathways, which potentially leads to cognitive deterioration present at certain stages of PD.

Supported by SAF2014-52582-R and SAF2014-6144-EXP A. Canerina-Amaro holds a fellowship from ACIISI. Se agradece la financiación concedida a la ULL por la Agencia Canaria de Investigación, Innovación y Sociedad de la Información, cofinanciada en un $85 \%$ por el Fondo Social Europeo

Neurodegeneration, Cellular model, Parkinson's Disease, Lipid Rafts, Alpha-synuclein.

\section{P1-25}

\section{ALTERED SYSTEMIC EHSP72 LEVELS AND NEUTROPHIL FUNCTION IN PATIENTS WITH OSTEOARTHRITIS.}

Isabel Gálvez ${ }^{(1)}$, María Dolores Hinchado ${ }^{(1)}$, Leticia MartínCordero ${ }^{(2)}$, Jorge Guerrero ${ }^{(3)}$, Silvia Torres-Piles ${ }^{(4)}$, Eduardo Ortega ${ }^{(1)}$

(1) Dpto. Fisiología (Grupo Inmunofisiología). Fac. Ciencias. UEX, Badajoz, Spain; ${ }^{(2)}$ Dpto. Enfermería (Grupo Inmunofisiología). Centro Universitario de Plasencia. UEX, Plasencia, Spain; ${ }^{(3)}$ Dpto. Enfermería. Facultad de Medicina. UEX, Badajoz, Spain; ${ }^{(4)}$ Dpto. Terapéutica Médico-Quirúrgica (Grupo Inmunofisiología). Fac. Medicina. UEX, Badajoz, Spain

Introduction: Osteoarthritis (OA) has predominantly been considered a non-inflammatory degenerative arthropathy leading to loss of cartilage, but today a growing number of evidences consistently suggest that several immunological and stress processes can also be altered in this pathology. In fact, aged patients with OA frequently refer a higher susceptibility to infections. Since neutrophils are the main circulating cells involved in the innate immune response against pathogens, and their function can be mediated by the stress protein $\mathrm{Hsp} 70$ (which has recently been reported to have a role in the pathophysiology of OA). 
Purpose: The present investigation was to evaluate potential alterations in the phagocytic and microbicidal activity of circulating neutrophils and in the concentration of eHsp72 in blood from OA patients.

Methods: Eleven patients diagnosed with primary OA according to the American College of Rheumatology (ACR) criteria (mean age 72,23 years old) and eight age-matched (mean age 67 years old) healthy volunteers (control group) were enrolled in the study. Serum eHsp72 levels were measured by ELISA. The phagocytic and oxygen-dependent microbicide capacities of circulating neutrophils were evaluated by flow cytometry.

Results: OA patients showed significantly high circulating levels of the stress protein eHsp72 along with a reduction in the phagocytic and microbicide capacities of neutrophils in relation to the age-matched healthy control group.

Conclusion: An immunoneuroendocrine dysregulation affecting systemic stress mediators, such as eHsp72, as well as the function of innate immune cells underlies OA. This dysregulation can contribute to an altered feedback between the innate/ inflammatory and stress responses in this pathology.

This investigation has been partially supported by Gobierno de Extremadura-FEDER (GR 15041) and an agreement UEX-"Balneario el Raposo" (036/15)

Osteoarthritis, Immunology, Innate immunity, Stress, eHsp72, Neutrophils

\section{P1-26}

AQUEOUS AND METHANOL EXTRACTS OF IBERVILLEA SONORAE POSSESS RELAXANT ACTIONS IN RABBIT SMALL INTESTINE: ROLE OF CALCIUM

Ana Del Carmen Susunaga-Notario (1), Raquel Forcén (1), Francisco Javier Alarcón-Aguilar ${ }^{(2)}$, María Divina Murillo ${ }^{(1)}$, Laura Grasa $(1,3,4)$

(1) Departamento de Farmacología y Fisiología, Facultad de Veterinaria, Universidad de Zaragoza, Zaragoza. Spain. ${ }^{(2)}$ Instituto de Investigación Sanitaria de Aragón (IIS-Aragón), Zaragoza. Spain. ${ }^{(3)}$ Instituto Agroalimentario de Aragón - IA2(Universidad de Zaragoza - CITA), Zaragoza. Spain

Background: Ibervillea sonorae (I. sonorae), or "wareque" (Cucurbitaceae) is a plant distributed in semi-arid areas in Mexico and it is widely used in Mexican traditional medicine as a topical antibiotic, cathartic, antirheumatic, hypoglycaemic substance and to treat stomach ulcers. However, the effects of I. sonorae on intestinal motility are totally unknown.

Objectives: The aim of the present study was to determine the effects of aqueous $\left(\mathrm{H}_{2} \mathrm{O}\right)$ and methanol $(\mathrm{MeOH})$ extracts from dried root of $I$. sonorae on the intestinal motility of rabbit ileum and evaluate the role of $\mathrm{Ca}^{2+}$ in these effects.

Methods: Whole segments of rabbit ileum were suspended in the direction of longitudinal muscle fibres in a thermostatically controlled organ bath, containing Krebs solution, at $37^{\circ} \mathrm{C}$ and gassed with $95 \% \mathrm{O}_{2}$ and $5 \% \mathrm{CO}_{2}$. Segments were connected to an isometric transducer and mechanical activities were recorded and analysed with a computer program. To study the effect of $\mathrm{H}_{2} \mathrm{O}$ and
$\mathrm{MeOH}$ extracts, we added $\mathrm{H}_{2} \mathrm{O}$ or $\mathrm{MeOH}$ extracts (1.2$3.2 \mathrm{mg} \mathrm{mL}^{-1}$ ) every $5 \mathrm{~min}$ and the cumulative concentrationresponse curves were performed.

In order to study the role of $\mathrm{Ca}^{2+}$ in $\mathrm{H}_{2} \mathrm{O}$ or $\mathrm{MeOH}$ extracts effects on the spontaneous contractions, Bay K8644, thapsigargin, trifluoperazine or verapamil were added to the bath, 15 min before $\mathrm{H}_{2} \mathrm{O}$ or $\mathrm{MeOH}$ extracts.

Results: The $\mathrm{H}_{2} \mathrm{O}$ and $\mathrm{MeOH}$ extracts evoked a concentrationdependent relaxation in the longitudinal smooth muscle of rabbit ileum. The incubation with Bay K8644 (a L-type $\mathrm{Ca}^{2+}$ channel activator), thapsigargin (an inhibitor of sarcoendoplasmic reticulum $\mathrm{Ca}^{2+}$ ATPase) or trifluoperazine (an inhibitor of the calcium-calmodulin complex) reverted the relaxation evoked by the $\mathrm{H}_{2} \mathrm{O}$ extract. The incubation with verapamil (a voltage dependent $\mathrm{Ca}^{2+}$ channels inhibitor) increased the relaxation induced by the $\mathrm{H}_{2} \mathrm{O}$ extract. The incubation with Bay K8644, thapsigargin, trifluoperazine or verapamil did not modify the relaxation evoked by the $\mathrm{MeOH}$ extract.

Conclusion: The $\mathrm{H}_{2} \mathrm{O}$ and $\mathrm{MeOH}$ extracts of $I$. sonorae possess relaxant actions in the rabbit small intestine. The relaxant effects of the $\mathrm{H}_{2} \mathrm{O}$ extract would be mediated by intracellular and extracellular $\mathrm{Ca}^{2+}$. The relaxant effects of the $\mathrm{MeOH}$ extract would not be mediated by $\mathrm{Ca}^{2+}$.

Ibervillea sonorae; rabbit, ileum, intestinal motility, Calcium

$\mathrm{P} 1-27$

DIFFERENT INTRACELLULAR PATHWAYS ARE INVOLVED IN THE MODULATION OF THE SEROTONIN TRANSPORTER BY NODS ACTIVATION IN CACO-2 CELLS

Elena Layunta ${ }^{(1)}$, Eva Latorre ${ }^{(2)}$, Rosario Alcutén ${ }^{(1)}$, Marta Castro ${ }^{(1)}$, Belén Gros ${ }^{(3)}$, Raquel Vicente ${ }^{(3)}$, María Pilar Arruebo ${ }^{(1)}$, Miguel Angel Plaza (1), Jose Emilio Mesonero ${ }^{(1)}$

(1) Departamento Farmacología y Fisiología, Facultad de Veterinaria, Instituto de Investigación Sanitaria de Aragón (IIS), Universidad de Zaragoza, Zaragoza. Spain. Instituto Agroalimentario de Aragón - IA2- (Universidad de Zaragoza CITA), Zaragoza, Spain., Zaragoza, Spain; ${ }^{(2)}$ RNA - Mediated Mechanisms of Disease. University of Exeter Medical School. Exeter. United Kingdom., Exeter, United Kingdom; ${ }^{(3)}$ Hospital Universitario Miguel Servet. Zaragoza. Spain., Zaragoza, Spain

Introduction: Innate immune system is the first defensive barrier in the intestinal epithelium, which includes some receptors (TLRs and NODs) that recognize microbiota to develop different responses. NOD1 and NOD2 are intracellular pattern recognition receptors involved in the recognition of peptidoglycan fragments from Gram-positive and Gramnegative bacteria. These receptors would have been involved in Inflammatory Bowel Diseases (IBD) due to a dysfunctional recognition of the microorganisms.

In this context, intestinal serotonergic system, which communicates nervous and immune system by serotonin (5-HT) seems to be altered in IBD with an increase of extracellular 5-HT availability. Intestinal 5-HT availability is regulated by the serotonin transporter (SERT), responsible for 5-HT reuptake in enterocytes. 
Aims \& Methods: The aim of this work has been to determinate whether SERT activity and expression is modified by NOD1 and NOD2 activation. The human enterocyte-like cell line Caco-2/TC7 was used as in vitro model. SERT activity was determined by 5 -HT uptake measurement. SERT, NOD1, and NOD2 mRNA expression were measured by RT-qPCR; and the corresponding protein expression was quantified by western blot, using specific antibodies.

Results: Both, NOD1 and NOD2 activation decreased SERT activity and expression in Caco-2 cells, by different mechanisms. NOD1 inhibitory effect seems to be mediated by ERK pathway, while NOD2 appears to activate RIP2 intracellular signaling pathway. Since SERT inhibition may result in increased extracellular 5HT levels, 5-HT effect on NODs expression was assayed, showing that NOD1 and NOD2 expression is decreased by 5 -HT in Caco-2 cells.

Conclusion: NOD1 and NOD2 activation may regulate intestinal 5-HT availability throughout different intracellular pathways in Caco-2 cells. In addition, serotonin also inhibits NOD expression, probably in a negative feed-back mechanism. Thus, this study confirms the interaction between serotonergic system and NOD receptors in the intestinal epithelium, which might contribute to intestinal homeostasis and pathological processes mediated by serotonin.

In Memoriam: This work is dedicated to the late Professor Anabel Alcalde, who started this research line.

Supported by MICINN-ERDF (BFU2010-18971), DGA-ESF (B61), Zaragoza University (UZ2014-BIO-03), and ARAINF (2012/0567)

NOD1, NOD2, 5-HT, CACO-2 CELLS, INTESTINE

P1-28

ANTIPROLIFERATIVE AND ANTIOXIDANT PROPERTIES OF ROSA CANINA PHYTOCHEMICALS ON CACO-2 CELLS

Sonia Gascón ${ }^{(1)}$, Sandra Jimenez ${ }^{(2)}$, Asunción Luquin ${ }^{(2)}$, María Ángeles Navarro ${ }^{(3)}$, Mariano Laguna (4), Carmen AncinAzpilicueta ${ }^{(2)}$, María Jesús Rodríguez-Yoldi ${ }^{(1)}$

(1) Departamento de Farmacología y Fisiología. CIBERobn. Facultad de Veterinaria. Universidad de Zaragoza, Zaragoza, Spain; ${ }^{(2)}$ Departamento de Química Aplicada. Universidad Pública de Navarra, Pamplona, Spain; ${ }^{(3)}$ Departamento de Bioquímica y Biología Molecular. Facultad de Veterinaria. Universidad de Zaragoza, Zaragoza, Spain; ${ }^{(4)}$ Departamento de Química Inorgánica. Facultad de Ciencias. Universidad de Zaragoza, Zaragoza, Spain

The commercial development of plants as sources of antioxidant that can be used to enhance the properties of foods, for nutritional purposes and preservation as well as for prevention of oxidant related diseases like cancer, it is the goal we perform has led to the present study. The in vitro antiproliferative and antioxidant effect of vitamin $\mathrm{C}$ (fraction 1), neutral polyphenols (fraction 2: myricetin, rutin, catechin and quercetin) and acidic polyphenols (fraction 3: vanillic acid, cafeic acid, syringic acid, gallic acid, ellagic acid and protocatechuic acid) of Rosa canina hips on human colon cancer cell lines (Caco-2) was studied. All extracts showed high cytotoxicity after $72 \mathrm{~h}$, both low and high concentrations. The flow cytometry analysis revealed that the extracts produce disturbances in the cell cycle resulting in a concomitant cell death by an apoptotic pathway. Changes in the redox status of Caco-2 cells in response to Rosa canina hips was determined. Cells were exposed to hydrogen peroxide in presence of plant fractions and the production of Reactive Oxygen Species (ROS) was decreased significantly. Therefore, our data show that the phytochemicals of rosehip are a powerful antioxidant that produces an antiproliferative effect in Caco- 2 cells. These results predict a promising future for Rosa canine in functional food science and more specifically natural products and their potential chemotherapeutic uses addressed for colorectal carcinoma.

Rosa canina, phytochemicals, cell viability, ROS, Caco-2 cells

P1-29

EFFECTS OF TOLL-LIKE RECEPTORS 2 AND 4 INHIBITION ON INTESTINAL CONTRACTILITY AND SEROTONERGIC SYSTEM OF MOUSE ENTERIC NERVOUS SYSTEM

Eva Latorre ${ }^{(1)}$, Ilaria Marsilio ${ }^{(2)}$, Valentina Caputi ${ }^{(2)}$, Elena Layunta $^{(3)}$, Silvia Cerantola ${ }^{(2)}$, Jose Emilio Mesonero ${ }^{(3)}$, Maria Cecilia Girón ${ }^{(2)}$

(1) RNA - Mediated Mechanisms of Disease. University of Exeter Medical School, Exeter, United Kingdom; ${ }^{(2)}$ Department of Pharmaceutical and Pharmacological Sciences, University of Padova, Padova, Italy; ${ }^{(3)}$ Departamento Farmacología y Fisiología, Facultad de Veterinaria, Instituto de Investigación Sanitaria de Aragón (IIS), Universidad de Zaragoza. Instituto Agroalimentario de Aragón - IA2- (Universidad de Zaragoza - CITA), Zaragoza, Spain

Introduction: Serotonin (5-HT) is a key neurotransmitter involved in maintaining gut homeostasis by modulating physiological functions, such as neuromuscular contractility, peristaltic and secretory reflexes and visceral sensitivity. Microbes influence host physiology either directly through their metabolic products or indirectly interacting with microbial associated molecular patterns receptors such as Toll-Like Receptors (TLRs). In gastrointestinal functional disorders (e.g. irritable bowel syndrome), changes in microbiota composition are associated with symptoms as well as alterations to both gut and brain serotonin levels.

Aims \& Methods: The aim of the present study was to assess whether TLR2 and TLR4 signaling affects the serotoninergic system expression on the enteric nervous system (ENS) of mouse ileum and the related consequences in neuromuscular motor function. Male mice C57BL/6J (2 weeks old) were daily treated intraperitoneally with OxPAPC (a TLR2 and TLR4 inhibitor; $1.5 \mu \mathrm{g} / \mathrm{g}$ body weight) for 7 days. The expression of 5-HT receptors 1A, 2A, 2B, 3, 4 and 7, 5-HT transporter (SERT) and enzyme 
tryptophan hydroxylase-2 (THP2) was analyzed by qPCR and western blot in isolated longitudinal muscle-myenteric plexus preparations. Contractile activity of isolated ileum segments, mounted vertically in organ baths, was evaluated as changes in isometric muscle tension following carbachol cumulative addition $(0.1-100 \mu \mathrm{M})$ or electric field stimulation (EFS, $1-40 \mathrm{~Hz}$ ).

Results: OxPAPC-treated mice showed an altered serotonergic system. A significant increase of mRNA levels of THP2, the enzyme responsible for nervous 5-HT production, and SERT, responsible for 5-HT uptake, were found, whereas SERT protein expression was reduced. The expression of the 5-HT receptors 1A, 2A, 2B, 4 and $7 \mathrm{mRNA}$ was also enhanced. In parallel, an altered contractility was found after TLR2 and TLR4 inhibition. Receptormediated response to carbachol and frequency-response curve to EFS resulted significantly increased in OXPAPC-treated mice $(\operatorname{Emax}=+42 \pm 3.5 \%, p<0.01$ and $+88 \pm 10 \%$ at $10 \mathrm{~Hz}, p<0.01$, respectively).

Conclusion: Our study provides evidence that TLR2 and TLR4 signaling is critical for gut homeostasis. The defective activity of these innate immunity receptors affects both the serotonergic system expression on the ENS and the intestinal neuromuscular motor function. The direct or indirect involvement of the serotoninergic system on gut dismotility remains to be elucidated.

In Memoriam: This work is dedicated to the late Professor Anabel Alcalde.

TLR2, TLR4, ENS, MOTILITY, 5-HT

P1-30

PROTOCATECHUIC ACID PREVENTS INFLAMMATION AND FIBROSIS, AND REGULATES THE SPHINGOSINE KINASE 1/SPHINGOSINE 1-PHOSPHATE SIGNALING PATHWAY IN AN ANIMAL MODEL OF COLITIS

Irene Crespo ${ }^{(1)}$, Beatriz San Miguel ${ }^{(2)}$, Valentín Manuel López ${ }^{(2)}$, Diana Isabel Sánchez ${ }^{(2)}$, Barbara Gonzalez-Fernandez ${ }^{(2)}$, Jose Luis Mauriz $^{(3)}$, Juan Ortiz De Urbina ${ }^{(4)}$, Javier González-Gallego (3), Mar Almar ${ }^{(2)}$, Maria Jesus Tuñon ${ }^{(3)}$

(1) IBIOMED and CIBERehd, León, Spain; (2) IBIOMED, León, Spain; ${ }^{(3)}$ IBIOMED and CIBEREHD, Leon, Spain; ${ }^{(4)}$ Servicio de Farmacia, Complejo Asistencial de León, León, Spain

Ulcerative colitis (UC) and Crohn's disease (CD) are the two major forms of inflammatory bowel disease (IBD) and are characterized by non-specific inflammation and intestinal tissue damage. Recent studies have shown that the inflammatory/oxidative and sphingolipid pathways are essential mechanisms that regulate the development of intestinal tissue damage and fibrosis. Sphingosine-1-phosphate (S1P), a pleiotropic bioactive lipid mediator, and sphingosine kinase 1 (SphK1), have now emerged as key regulators of numerous cellular processes involved in inflammation and cancer. The aim of this study was to assess the role of protocatechuic acid (PCA), a phenolic compound commonly found in many edible vegetables, on the mechanisms involved in inflammatory/oxidative S1P/Sphk1 pathways in a murine model of colitis. Colitis was induced in BALB/c mice by intracolonic administration of 2,4,6-trinitrobenzenesulfonic acid (TNBS) $(2 \mathrm{mg} / 0.1 \mathrm{~mL}$ of ethanol $50 \%$ ). PCA was dissolved into saline and administered at two different doses ( 30 or $60 \mathrm{mg} / \mathrm{kg}$ body wt) after colitis induction by rectal route daily up to the end of the study, at day 5. After sacrifice, expression of different markers was measured by qRT-PCR, Western blot and ELISA. Increased expression of proinflammatory and fibrogenic cytokines (IL-6 and TGF- $\beta$ ), in agreement with microscopic findings (Masson Trichrome Staining), was found in animals with TNBS-induced colitis. This effect was partially abolished by PCA administration in a dose dependent manner. Antioxidant status, evaluated by expression of superoxide dismutase (SOD) and catalase (CAT), was significantly reduced in the group with colitis, while PCAtreated animals recovered levels similar to the control group in a dose-dependent manner. Regarding the sphingolipid pathway, expression of SphK1, S1P and the receptor S1PR1 increased significantly in TNBS-treated mice, and this effect was abrogated by PCA administration. Recently has been reported that persistent activation of STAT3 by the Sphk1/S1P signaling pathway is observed in chronic intestinal inflammation and colitis-associated colorectal cancer. Interestingly, PCA treatment prevented the marked overexpression of phospho-STAT3 in animals with colitis. Our findings suggest that PCA treatment not only attenuates the outcome of TNBS-induced colitis by reducing the inflammatory response but also by downregulating the SphK1/S1P pathway. This molecule may be an interesting candidate to reduce the risk of inflammation and cancer progression in patients with IBD. Supported by Plan Nacional RTA 2012-00022-C02-02

Sphingosine Kinase, Sphisgosine-1-Phosphate, Protocatechuic acid, Inflammation, Fibrosis

P1-31

INVOLVEMENT OF TRPV2 IN COLONIC PROPULSION. "IN VIVO" STUDY IN AN EXPERIMENTAL MOUSE MODEL

Ester Murillo ${ }^{(1)}$, Miguel Angel Plaza ${ }^{(2)}$, Jesús María Muñoz ${ }^{(1)}$, Aurora Gimeno ${ }^{(1)}$, José Emilio Mesonero ${ }^{(2)}$, Laura Grasa ${ }^{(2)}$, María Pilar Arruebo ${ }^{(2)}$, Marta Castro ${ }^{(2)}$

(1) Departamento de Farmacología y Fisiología, Universidad de Zaragoza. Zaragoza. Spain, Zaragoza, Spain; ${ }^{(2)}$ Departamento de Farmacología y Fisiología. Instituto de Investigación Sanitaria Aragón. Instituto Agroalimentario de Aragón - IA2- (Universidad de Zaragoza - CITA). Zaragoza. Spain, Zaragoza, Spain

Introduction: Transient receptor potential vanilloid subtype 2 (TRPV2) is a cationic channel belonging to the superfamily of TRP channels. The role of TRPV2 is complex as it responds to mechanical forces and to several exogenous and endogenous substances such as hormones, growth factors, chemotactic peptides, lysophospholipids and cannabinoids. In the gastrointestinal tract TRPV2 expression has been observed in the inhibitory motor neurons of the myenteric plexus from mouse stomach, contributing to receptive relaxation and to gastric emptying. It has also been demonstrated its location in intrinsic primary afferent neurons (IPANs) or in inhibitory motor neurons modulating intestinal motility. However, the putative role of TRPV2 in the motor regulation of the distal parts of digestive tract remains unknown. 
Aim and Methods: The aim of the present study was to investigate the role of the TRPV2 channel in the regulation of the distal colonic motility, using in vivo techniques in mice.

After an overnight fasting period and under anesthesia with isoflurane, one of the following agents was intracolonically administered in a final volume of $100 \mu \mathrm{l}$ : the TRPV2 agonist probenecid $(100 \mathrm{mg} / \mathrm{kg})$, the TRPV2 antagonist tranilast $(10 \mathrm{mg} / \mathrm{kg}$ ), both together or their common solvent (carboxymethylcellulose $0.5 \%$ ). After $30 \mathrm{~min}$, colonic transit was measured through the time taken to expel a glass bead $(2 \mathrm{~mm}$ diameter) previously inserted anally $2 \mathrm{~cm}$ into the distal colon, under anesthesia. Distal colon samples were collected immediately after euthanasia to localize the TRPV2 receptor by immunofluorescence. All procedures were approved by the Ethics Committee for Animal Experiments at the University of Zaragoza (PI38/12).

Results and Conclusion: The results obtained showed that the intracolonic administration of the TRPV2 agonist probenecid decreased the time of expulsion of the glass bead $(p<0.05)$. On the other hand, the administration of the TRPV2 antagonist tranilast did not modify this parameter. Tranilast abolished the increase in the colonic transit induced by probenecid when they were administered together. In addition, immunoreactivity for TRPV2 was observed in neurons of the myenteric plexus as well as in the cytoplasm and in the brush border of epithelial cells of the colonic mucosa. Our results suggest that TRPV2 is involved in the regulation of distal colonic transit in mice.

Supported by DGA-FSE (B61, CTPP10/11), Universidad de Zaragoza (UZ2014-BIO-03) and Universidad de Zaragoza-Fundación Ibercaja (JIUZ-2015-BIO-02).

Colonic motility, colonic transit, TRPV2, transient receptor potential channel, mouse

\section{$\mathrm{P} 1-32$}

\section{ROLE OF GRANZYME A IN THE REGULATION OF SMALL} INTESTINAL MOTILITY IN MICE

José Luis Bueno ${ }^{(1)}$, Eduardo Moreo ${ }^{(1)}$, Marta Sofia Valero ${ }^{(2)}$, Llipsy Santiago (3), Maykel Arias (3), Elena Layunta ${ }^{(4)}$, Ana Cristina Calvo ${ }^{(5)}$, Julián Pardo ${ }^{(3)}$, María Pilar Arruebo ${ }^{(4)}$, Miguel Angel Plaza ${ }^{(4)}$, Marta Castro ${ }^{(4)}$

(1) Departamento de Anatomía, Embriología y Genética Animal, Zaragoza, Spain; ${ }^{(2)}$ Facultad de Ciencias de la Salud. Universidad San Jorge, Zaragoza, Spain; ${ }^{(3)}$ Departamento de Bioquímica y Biología Molecular y Celular. IIS Aragón -Universidad de Zaragoza, Zaragoza, Spain; ${ }^{(4)}$ Departamento de Farmacología y Fisiología. IIS Aragón-IA2-Universidad de Zaragoza. Zaragoza. Spain., Zaragoza, Spain; ${ }^{(5)}$ Departamento de Anatomía, Embriología y Genética Animal. LAGENBIO-IA2-Universidad de Zaragoza, Zaragoza, Spain

Introduction: Granzyme A (gzmA) is a serin protease produced by immune cells that has been implied in apoptosis and inflammation. Nevertheless, nothing is known about its putative role in the regulation of physiological functions, such as intestinal motility.
Aim and Methods: The aim of this work was to study the possible involvement of gzmA on the regulation of intestinal motility through the evaluation of in vitro ileal motor responses induced by several contractile agents in mice. Adult (8-12 weeks) wild-type (wt) male C57Bl/6 and gzmA knockout $\left(\mathrm{gzmA}^{-1}\right)$ mice were humanly killed by cervical dislocation and intestine was extracted. Whole ileum segments were longitudinally mounted in an organ bath containing Ringer Krebs ( $\mathrm{pH} 7.4)$ at $37{ }^{\circ} \mathrm{C}$ continually gassed with carbogen $\left(5 \% \mathrm{CO}_{2}, 95 \% \mathrm{O}_{2}\right.$ ). Each segment was connected to an isometric force transducer. The following agents were added to the bath and incubated for a 3-min period: acetylcholine (ACh, $\left.10^{-7} \mathrm{M}\right), \mathrm{KCl}(80 \mathrm{mM})$, substance P (SP, $\left.10^{-7} \mathrm{M}\right), 17$-fenilprostaglandin $\mathrm{E}_{2}\left(17-\mathrm{fenil}-\mathrm{PGE}_{2}, 10^{-7} \mathrm{M}\right)$ and serotonin (5-HT, $\left.10^{-4} \mathrm{M}\right)$. After registering responses, the preparations were washed with Krebs. The effect of each agent was measured as integrated motor activity and it was expressed as $\mathrm{mN}$ / second.

Results: In the longitudinal ileal smooth muscle segments obtained from wt mice, ACh $\left(10^{-7} \mathrm{M}\right), \mathrm{KCl}(80 \mathrm{mM})$ and 5HT $\left(10^{-4} \mathrm{M}\right)$ evoked contractions reaching a maximal response of $150.0 \pm 14.1 \mathrm{mN} / \mathrm{s}, 120.3 \pm 11.2 \mathrm{mN} / \mathrm{s}$ and 114.4 $\pm 7.1 \mathrm{mN} / \mathrm{s}$ respectively. In the segments from gzm A ${ }^{-/}$animals these agents induced contractile responses with a mechanical integrated activity being $\approx 20-25 \%$ lower (120.6 $\pm 9,1 \mathrm{mN} / \mathrm{s}, 94.3 \pm 3,2 \mathrm{mN} / \mathrm{s}$ and $94.8 \pm 11 \mathrm{mN} / \mathrm{s}$ respectively) than those obtained in wt animals. In contrast, we did not observed differences in the contractile responses evoked by $\mathrm{SP}\left(10^{-7} \mathrm{M}\right)$ and 17 -fenil-PGE $2\left(10^{-7} \mathrm{M}\right)$.

Conclusion: Our results suggest that granzyme A may be involved in the regulation of ileal motility, taking part at least in the contractions evoked by depolarization or by modulators such as ACh or 5-HT.

Supported by DGA-FSE (B61), Universidad San Jorge and Universidad de Zaragoza-Fundación Ibercaja (JUZZ-2015-BIO-02).

Granzyme A, intestinal motility, organ bath, mouse

P1-33

ROCK TEA NORMALIZES AUTOPHAGY BIOMARKERS IN A MURINE MODEL OF COLITIS

Eduardo Moreo ${ }^{(1)}$, José Luis Bueno ${ }^{(1)}$, Marta Sofía Valero ${ }^{(2)}$, Mariano Ramón Giménez ${ }^{(2)}$, María Pilar Arruebo ${ }^{(3)}$, Miguel Ángel Plaza ${ }^{(3)}$, Ana Cristina Calvo ${ }^{(1)}$, Marta Castro (3)

(1) Departamento de Anatomía, Embriología y Genética Animal. LAGENBIO-IA2-Universidad de Zaragoza, Zaragoza, Spain; (2) Facultad de Ciencias de la Salud. Universidad San Jorge, Zaragoza, Spain; ${ }^{(3)}$ Departamento de Farmacología y Fisiología. IIS Aragón-IA2-Universidad de Zaragoza. Zaragoza. Spain., Zaragoza, Spain

Introduction: Ulcerative colitis is a prevalent disease characterized by mucosal inflammation at colon and rectum. In the last years, the hypothesis of commensal bacteria of the intestinal lumen having an important role in the development of the disease has been gaining adepts. 
Autophagy is a physiological process activated in response to cellular stress such as starvation, inflammation and interaction with bacteria, and is altered in colitis.

Jasonia glutinosa (rock tea) is a plant found in some regions of Spain and southern France. It has been traditionally used to treat gastrointestinal diseases but its therapeutical role has not been scientifically validated. We have previously shown that rock tea reduces clinical score and inflammation induced by dextran sodium sulphate (DSS).

Objective: The main objective of this work was based on the study of the potential role of Jasonia glutinosa as a modulator of the dysregulation of autophagy induced in an animal model of colitis.

Materials and methods: C57Bl/6 male mice aged 8-12 weeks were used. Animals received for 20 days an extract of rock tea $(50 \mathrm{mg} / \mathrm{kg},-\mathrm{RT}$ group-), a standard drug for the treatment of ulcerative colitis (sulfasalazine, $100 \mathrm{mg} / \mathrm{kg},-\mathrm{S}$ group-) or their solvent (water,-control and DSS groups-). At day 10, colitis was induced in RT, S and DSS groups by the administration of $2.5 \%$ of DSS during 7 days. After sacrifice distal colon was removed. Homogenization of the tissue was carried out and autophagy biomarkers $\mathrm{BCN} 1, \mathrm{E} 2 \mathrm{~F} 1, \mathrm{LC} 3 \mathrm{~b}$ and $\mathrm{LC} 3 \mathrm{a} / \mathrm{b}$ ratio were analized by Western blot.

Results and conclusion: DSS led to a significant increase in $\mathrm{BCN} 1$. In contrast, both rock tea and sulfasalazine normalized the expression of this marker when compared to control. In rock tea treated mice E2F1, LC3b levels and $\mathrm{LC} 3 \mathrm{a} / \mathrm{b}$ ratio showed a tendency to normalization to control levels. These preliminary findings show that Jasonia glutinosa may ameliorate the dysregulation of autophagy induced by DSS in this animal model, suggesting promising therapeutic properties for the treatment of colitis.

This work was supported by Grant PI14/00947 from Fondo de Investigación Sanitaria of Spain, DGA-FSE (B61, CTPP10/11), Universidad de Zaragoza (UZ2014-BIO-03), Universidad de Zaragoza-Fundación Ibercaja (JIUZ-2015-BIO-02) and Universidad San Jorge.

Rock tea, colitis, autophagy, mouse

P1-34

ENVIRONMENTALLY RELEVANT DOSES OF XENOESTROGENS MODULATE MOUSE PANCREATIC BCELL FUNCTION

Sabrina Villar-Pazos (1), Juan Martinez-Pinna ${ }^{(2)}$, Manuel Castellano-Munoz ${ }^{(1)}$, Paloma Alonso-Magdalena ${ }^{(1)}$, Ivan Quesada $^{(1)}$, Angel Nadal ${ }^{(1)}$

(1) CIBER de Diabetes y Enfermedades Metabólicas Asociadas (CIBERDEM)-Universidad Miguel Hernández, Elche, Spain;

(2) Departamento de Fisiología, Genética y MicrobiologíaUniversidad de Alicante, Alicante, Spain

Background: Environmental endocrine-disrupting chemicals (EDCs) refer to a broad group of exogenous chemicals, or combination of them, that perturb hormonal signalling. It is well known that exposure to this compounds, most of which are originate for industrial purposes, are related to increased incidence of a huge subset of endocrine deregulations and pathologies. Among the EDCs are included xenoestrogens (e.g. bisphenol A), phthalates, herbicides and pesticides (e.g. DDT). Based on epidemiological studies and animal model research, a growing number of EDCs seem to be implicated in the disruption of glucose and lipid homeostasis, and thus the exposure to these compounds is considered a risk factor for the development of type 2 diabetes (T2D) and obesity. Xenoestrogens alter estrogenic signaling by binding estrogen receptors (ER). Some xenoestrogens have been shown to impair glucose homeostasis by affecting both insulin sensitivity and pancreatic $\beta$-cell function. However the underlying molecular mechanisms are still under research.

Aims and methods: We have investigated the effect of the persistent exposition to low doses of specific xenoestrogen on pancreatic $\beta$-cell function. For this purpose, we recorded intracellular calcium signals using fura- 2 fluorimetry. Additionally, whole cell currents were measured by patch-clamp technique and the exocytotic responses of $\beta$-cells were monitored by changes in single-cell capacitance. Finally, mRNA expression levels of different channel subunits were determined using quantitative RT-PCR.

Results and conclusions: Incubation with low doses of xenoestrogen promoted changes in intracellular calcium responses elicit by glucose as well as non-metabolic depolarizing stimuli. Whole-cell patch-clamp recordings suggested that these alterations were related to the modulation of macroscopic currents in treated pancreatic $\beta$-cells. Pharmacological approach, using specific current blockers, allowed to evaluate the specific contribution of the main current types, suggesting that non-L-type calcium current was altered. Finally, chronic exposition to exogenous estrogen regulated exocytotic responses in $\beta$-cells.

Funding: SAF2014-58335-P, PROMETEOII/2015/016 Generalitat Valenciana

Pancreatic $\beta$-cell, xenoestrogens, calcium signal

P1-35

FUNCTIONAL AND MORPHOLOGICAL ADAPTATIONS IN THE PANCREATIC A-CELL DURING PREGNANCY IN MICE

Cristina Quesada-Candela, Paloma Alonso-Magdalena, Ivan Quesada, Angel Nadal

CIBER de Diabetes y Enfermedades Metabólicas Asociadas (CIBERDEM)-Universidad Miguel Hernández, Elche, Spain

During pregnancy, the mother undergoes major hormonal and metabolic changes to meet the energy requirements of the growing fetus. Therefore, important functional and structural adaptations occur in the pancreatic beta-cells to maintain an optimal glucose homeostasis. These adaptations arise to compensate for the increased insulin resistance. However, little is known about the adaptations of alpha-cells during pregnancy. Based on the recent findings of our group, showing the 
adaptations of the alpha-cell in other model of insulin resistance (obesity), we are studying morphofunctional features of pancreatic alpha-cells during pregnancy in mice.

Our groups of study are female mice non-pregnant, as control group, and the gestational days G12.5, G15.5 and G18.5. Non-fasting plasma glucagon and insulin concentrations were determined by ELISA. Intracellular calcium signaling was examined by confocal microscopy in the control group and day G15.5. To analyze the $\mathrm{Ca}^{2+}$ mobilization, individual cells within freshly isolated islets were loaded with Fluo-4 and monitored. We used two concentrations of glucose, a stimulatory $(0.5 \mathrm{mM})$ and a non-stimulatory $(5.6 \mathrm{mM})$ concentration for the alpha-cells. $\mathrm{A} \mathrm{Ca}^{2+}$ oscillation was defined as a rapid increase in the intracellular $\mathrm{Ca}^{2+}$ concentration higher than twice of the background signal. We analyzed the area under the curve (AUC), as a global indicator of the mobilization of calcium during the stimulus, in the last $5 \mathrm{~min}$. of each one.

In the case of the non-fasting glucagon concentrations, we have observed a significant diminution in gestational days G12.5 and G18.5. Alternatively, non-fasting insulin concentration was elevated in G15.5, but not with statistical significance. The analysis of the intracellular calcium signaling showed that the percentage of alpha-cells not inhibited by the $5.6 \mathrm{mM}$ glucose stimulus is higher in the G15.5 mice, than in non-pregnant mice. Additionally, the AUC is significantly increased in G15.5 during the $0.5 \mathrm{mM}$ glucose stimulus.

Funding: SAF2014-58335-P, PROMETEOII/2015/016 (Generalitat Valenciana) and BFU2013-42789-P.

Pregnancy, pancreas, islet of Langerhans

P1-36

\section{EFFECTS OF PRENATAL EXPOSURE TO XENOESTROGENS IN MOUSE PANCREATIC B-CELL DEVELOPMENT}

Talía Boronat Belda, Marta García Arévalo, Esther Fuentes, Ivan Quesada, Angel Nadal, Paloma Alonso Magdalena

Centro de Investigación Biomédica en Red de Diabetes y Enfermedades Metabólicas Asociadas CIBERDEM-Universidad Miguel Hernández, Elche, Spain

Background: It is widely known that insults during early life may alter growth and maturation of key tissues and organs and this is related to the onset of adult metabolic disorders. Particularly, maternal nutrition is considered an important factor for $\beta$-cell development and maturation of pancreatic $\beta$-cell function. Here we explore if other factors like maternal hormone environment during pregnancy may also alter the development of pancreatic $\beta$ cell mass.

Objectives: The aim of this work was to study the consequences of the exposure to an abnormal estrogenic environment during gestation on pancreatic $\beta$-cell mass, together with $\beta$-cell proliferation and $\beta$-cell apoptosis. For this purpose pregnant animals were exposed to bisphenol-A (BPA), a widespread xenoestrogen that can disrupt estrogen signaling by binding estrogen receptors.

Materials and Methods: OF1 pregnant mice were treated with either vehicle (Control), 17- $\beta$ estradiol (E2) $(10 \mu \mathrm{g} /$ $\mathrm{kg}$ /day) or bisphenol-A (10 or $100 \mu \mathrm{g} / \mathrm{kg} /$ day) during days 9 to 16 of gestation. Pancreatic $\beta$-cell mass, $\beta$-cell division and apoptosis were examined in male offspring at 1 month of age. The ethical committee of Miguel Hernandez University specifically approved this study (UMH-IB-AN-01-14, IB-PAM-01-15).

Results: We found an increase in the percentage of $\beta$-cell area relative to the total pancreas area, as well as enhanced pancreatic $\beta$-cell mass, in BPA and E2 male mice compared to control animals at 1 month of age. An increased pancreatic $\beta$-cell mass can be due to an increase of $\beta$-cell proliferation capacity, a decrease of apoptosis rate, or both. We quantified the number of BrdU-positive $\beta$-cells as an indicator of the proliferation rate and found that this parameter was increased in BPA mice compared to controls. No effects were observed in E2 mice. In addition, TUNEL staining revealed decreased $\beta$-cell apoptosis both in BPA and E2 mice.

Conclusions: These results suggest that the exposure to an abnormal estrogenic environment during pregnancy alter pancreatic $\beta$-cell mass in the offspring. We hypothesize that these early changes may affect the phenotype later in life.

Funding: SAF2014-58335-P, PROMETEOII/2015/016 Generalitat Valenciana, Sociedad Española de Diabetes CY1002IL.

Pregnancy, xenoestrogens, pancreatic $\beta$-cell mass, $\beta$-cell division

P1-37

ASSOCIATION OF PLASMA OMENTIN LEVELS WITH CARDIOVASCULAR PARAMETERS IN PATIENTS WITH METABOLIC SYNDROME

Marta Martín Rincón ${ }^{(1)}$, Roberto Ortíz-Regalón ${ }^{(1)}$, José Felipe Varona $^{(2)}$, Isabel Sánchez-Vera ${ }^{(1)}$

(1) Universidad San Pablo CEU, Boadilla del Monte, Madrid, Spain; ${ }^{(2)}$ Hospital HM Montepríncipe, Boadilla del Monte, Madrid, Spain

Introduction: Omentin is a recently identified adipocytokine mainly secreted by human visceral fat tissue. Omentine has been associated to anti-diabetic, anti-inflammatory, antiatherogenic and anti-bone loss functions. Lower plasma omentin levels have been related with pathogenesis of insulin resistance, type 2 diabetes, obesity and endothelial dysfunction. In spite of this, the pathophysiological role of omentin in Metabolic Syndrome (MetS) is not clear, and there are few studies in MetS subjects. The aim of this study is to investigate the association between plasma omentin levels and cardiovascular risk in patients with MetS.

Methods: 71 patients with MetS according to NCEP ATP III criteria were included (age: $49.96 \pm 8.71$ years). Plasma omentin levels were determined by enzyme-linked immunosorbent assay 
kit. Clinical and biochemical parameters (total cholesterol, cHDL, cLDL, triglycerides, abdominal obesity, systolic and diastolic blood pressure), data for evaluating arteriosclerotic carotid damage (intima-media thickness [IMT] $>0.9 \mathrm{~mm}$ ) and left ventricular hypertrophy $(\mathrm{LVH})$ were collected.

Results: Circulating omentin levels correlated with c-HDL $(r=0.411 ; p=0.001)$ and with adiponectin $(r=0.905 ; p=0.001)$. Patients with arterial hypertension $(n=21)$ had lower plasma omentin levels than patients with normal blood pressure $(n=49)$, $(9.36 \pm 7.54$ vs $14.84 \pm 10.12 ; p=0.023)$. There was no statically difference in omentin levels between patients with or without hypertriglyceridemia, abdominal obesity, impairment fasting glucose or low cholesterol HDL. There were no association between omentin and IMT $>0.9 \mathrm{~mm}$ or LVH presence.

Conclusions: In MetS subjects, low levels of plasma omentin are associated to arterial hypertension. However, there are no statically differences in plasma omentin concentrations between patients with or without arteriosclerotic carotid damage (IMT $>0.9 \mathrm{~mm}$ ) or LVH.

Omentin, Metabolic Syndrome, arterioesclerotic carotid damage

\section{P1-38}

ORAL MELATONIN IMPROVES THE SLEEP QUALITY AND INCREASES 6-SULFATOXYMELATONIN LEVELS IN PATIENTS WITH FIBROMYALGIA

$\mathrm{M}^{\mathrm{a}}$ Yolanda Castaño Blanco, María Garrido Álvarez, Ma Ángeles Gómez Zubeldia, Ana Beatriz Rodríguez Moratinos

Universidad de Extremadura (Facultad de ciencias). Dpto Fisiología., Badajoz, Spain

Background/aim: Fibromyalgia (FM) is a chronic widespread pain syndrome that is accompanied by fatigue and sleep disturbances, among other symptoms. It has been suggested that patients with FM present low circulatory melatonin levels, an indole with chronobiotic properties. The objective of this study was to assess the effect of different doses of melatonin on both sleep quality and 6-sulfatoxymelatonin (aMT6-s) levels in patients with FM.

Material and methods: The study was carried out in 36 patients diagnosed with severe FM. The study had a placebo-controlled design. Before starting the assay, a 10-day baseline period was left. Then, patients took different doses of melatonin $(3,6,9,12$, $15 \mathrm{mg} /$ day). Each dose was taken as a single pill at night during 10 consecutive days. Placebo (1 pill/day) was given during 10 days either before or between (washout period) melatonin doses. Actigraphic monitoring was used to record and display the temporal patterns of the individuals' activity and rest. Each subject wore on a wrist an actimeter that logged activity in basal conditions (10 days before the beginning of the assay) and throughout the entire assay. Subjective sleep quality was measured by The Pittsburgh Sleep Quality Index (PSQI). This questionnaire was filled in at the end of each period: basal, placebo, melatonin (different doses) and washout. First-morning void urines were collected the day after the end of each period. For the quantification of aMT6-s, a commercial ELISA kit was used.

Results: The administration of 9, 12 and $15 \mathrm{mg} /$ day of melatonin decreased significantly the sleep latency, whereas doses of 12 and $15 \mathrm{mg} /$ day caused a significant improvement in sleep efficiency, assumed sleep, actual sleep time, immobility, and total nocturnal activity. The PSQI showed significant improvements of sleep quality after the ingestion of 6,9 , 12 and $15 \mathrm{mg} /$ day of melatonin; similar doses of melatonin were effective in increasing aMT6-s levels.

Conclusion: The administration of $6,9,12$, and $15 \mathrm{mg} /$ day of melatonin during 10 days improved the sleep quality as well as aMT6-s levels in patients with FM. Therefore, melatonin might be a potential adjuvant therapy in FM.

Acknowledgments: Supported by Junta de Extremadura-Fondo Social Europeo de Desarrollo Regional (FEDER) grant BBB021- GR15051. Maria Garrido holds a research postdoctoral fellowship from Gobierno de Extremadura [jointly financed by the European Regional Development Fund (ERDF); ref. PO14013].

Fibromyalgia, melatonin, sleep, 6-sulfatoxymelatonin

P1-39

SENSITIZING EFFECTS OF MELATONIN ON DOCETAXEL TREATMENT IN HUMAN BREAST CANCER CELLS (MCF-7)

Javier Menéndez-Menéndez, Carolina Alonso-González, Alicia González-González, Alicia González, Samuel Cos, Carlos Martínez-Campa

Department of Physiology and Pharmacology, School of Medicine, University of Cantabria and Instituto de Investigación Valdecilla (IDIVAL), Santander, Spain

The pathogenesis and growth of breast cancer are linked to estrogens, which are considered key molecules in mammary carcinogenesis. About $80 \%$ of all breast cancers are estrogen receptorpositive (ER+) and, therefore, they respond well to hormonal therapy. Melatonin, the pineal gland hormone, has been shown by numerous in vivo and in vitro studies to have an oncostatic role on hormone-dependent tumors, especially on estrogen-dependent mammary tumors. The inhibitory action of melatonin on breast cancer cells is based on its interaction with either the synthesis of estrogens or the estrogen-signaling pathway. In the past few years, evidence of a broader spectrum of actions of melatonin as an antitumoral agent have arisen, thus, melatonin seems to also have therapeutic effects in several types of hormone-independent cancer, such as ovarian, leukemic, pancreatic, gastric and non-smallcell lung carcinoma. Clinical trials conclude that patients who received melatonin showed substantial improvements in tumor remission, survival and side effects of chemotherapy, which points to this indolamine as a potential adjuvant for chemotherapy to be considered.

Docetaxel (Taxotere) is a microtubule inhibitor agent (MIA) commonly used in breast cancer chemotherapy treatment. The main mechanism of its cytotoxicity is due to disruption of microtubule function leading to the inhibition of microtubule dynamics, particularly of those comprising the mitotic spindle apparatus, directly causing metaphase arrest. Since docetaxel (and all chemotherapy agents) causes undesirable side effects, development of resistance and changes in gene expression, the main goal of this work was to 
test if melatonin can modulate the effects of docetaxel in the estrogen-responsive MCF-7 human breast cancer cells. We found that pharmacological doses of docetaxel $(1 \mathrm{mM})$ induce changes in gene expression and that melatonin is capable to modulate those changes. Physiological doses of melatonin $(1 \mathrm{nM})$ potentiate the antiproliferative activity of subpharmacological doses $(0.1 \mathrm{nM}$ and $1 \mathrm{nM}$ ) of docetaxel and further stimulates the apoptosis triggered by this agent. Interestingly, pretreatment with melatonin sensitizes MCF-7 cells to subpharmacological doses of docetaxel and potentiates its antiproliferative effects.

Our findings suggest that melatonin may benefit cancer patients who are receiving chemotherapy and therefore, clinical trials using melatonin as an adjuvant therapy in breast cancer should be conducted.

This work was supported by grants for the Spanish Science Technology and Innovation Ministry (SAF2013-42012-P) and IDIVAL (APG/12).

P2-01

HIGH-FAT DIETS AND ITS INFLUENCE ON HEPATIC AMINOPEPTIDASES AS REGULATORS OF INTAKE AND FUNCTIONAL MARKERS IN AGED WISTAR RAT

Sara Jimenez Serrano, Isabel Prieto Gomez, Ana Belén Segarra Robles, Manuel Ramírez Sánchez, Germán Domínguez Vías

Área de Fisiología, Universidad de Jaén, Jaen, Spain

Obesity is pathologically due to disbalance between intake and energetic expenditure. It is considered a serious chronic disease characterized by excessive accumulation of adipose tissue to cause adverse health consequences, and among them the ectopic lipid deposition in the liver.

Objectives: The main goal of the study is to investigate the association between intake of high-fat diets (HFD) and obesity, together with hepatic variations developed by different degrees of dietary fatty acid saturation.

Methodology: Old male Wistar rats were classified in groups, based on its feeding, for 6 months with a standard diet (S), and isocaloric diets enriched with monounsaturated fatty acids supplemented with extra virgin olive oil (VOO), and enriched with saturated fatty acids supplemented with butter plus cholesterol (Bch). Liver tissues were removed to measure soluble and membrane bound aminopeptidase activities, determined fluorometrically using arylamides as substrates.

Results: The VOO group did not induce obesity in contrast to Bch group. In turn, the significant decrease of the intake taken place in Bch group was corresponded with highest values of soluble enkephalinase/hepatic tyrosyl aminopeptidase (TyrAP), involved in the metabolic and hedonic control of the appetite and body weight. The significant increase of membrane bound gammaglutamyl transferase (GGT) and soluble leucyl aminopeptidase (LeuAP) activities, excluding prolyl iminopeptidase (PIP), showed deterioration in the state of hepatic functionality on the Bch group. Even so, positive correlations were obtained between soluble and membrane fraction of hepatic PIP and pairing GGT/LeuAP activities after feeding period with HFD. Besides, PIP, just like GGT, displayed significant correlations with its plasma lipid profile.
Conclusion: A high intake of monounsaturated fatty acids is proposed like a dietary factor which could improve metabolic disorders caused by obesity, and this way to regulate as hedonic control as impact of liver diseases and oxidative stress. These results would determine future treatments for obesity.

Obesity, intake, high-fat diet, aminopeptidase, liver disease, functional marker.

P2-02

THE USE OF THE ACIDOPHILIC MICROALGA COCCOMYXA SP. (STRAIN ONUBENSIS) AS SAFE FOOD FOR ANIMALS

Francisco Navarro ${ }^{(1)}$, Eduardo Forján ${ }^{(2)}$, Zaida Moreno ${ }^{(2)}$, Miguel Ángel Castaño (3), Alberto Toimil (1), Miguel Ángel García-Sevillano $^{(2)}$, Marisa Sánchez ${ }^{(4)}$, Inés Garbayo ${ }^{(2)}$, Carlos Vílchez ${ }^{(2)}$, José María Vega ${ }^{(5)}$, Rosario Pasaro ${ }^{(6)}$

(1) Dept. de Biología Ambiental y Salud Pública, Facultad de Ciencias Experimentales, Universidad de Huelva, Huelva, Spain;

(2) CIDERTA and Dept. Química y Ciencias de los Materiales, Facultad de Ciencias Experimentales, Universidad de Huelva, Huelva, Spain; ${ }^{(3)}$ Complejo Hospitalario Universitario de Huelva, Juan Ramón Jiménez, Huelva, Spain; ${ }^{(4)}$ Hospital de Riotinto, Huelva, Spain; ${ }^{(5)}$ Dept. de Bioquímica Vegetal y Biología Molecular. Facultad de Química. Universidad de Sevilla, Sevilla, Spain; (6) Dept. de Fisiología, Facultad de Biología, Universidad de Sevilla, Sevilla, Spain.

Relevant information: The commercial interest of microalgae has increased during the past decades because its biomass is a rich source of a vast group of chemicals with applications in feed, food, nutritional, cosmetic, pharmaceutical and fuel industries. Up to date, microalgae used as food for humans and animals are marine or fresh water mesophilic species, however, extremophilic microalgae has been scarcely used. Coccomyxa sp. (strain onubensis) was isolated from the Tinto River (Huelva, Spain) water, which has $\mathrm{pH} 2.5$, and high concentrations of sulfur-compounds and heavy metals, including iron, copper, magnesium and aluminium. This microalga was grown in the lab autotrophically, in liquid minimum mineral medium and have the advantage that due to the low $\mathrm{pH}$ used the cultures are not easily contaminated by other microorganisms. C. onubensis dry biomass is rich in proteins $(44.60 \%)$ and dietary fibers $(15.73 \%)$, moderate in carbohydrates $(24.8 \%)$ and low in lipids $(5.4 \%)$ in which polyunsaturated fatty acids represent $65 \%(w / w)$ of the total fattys acid content. In addition the microalga has a pigment profile that is rich in carotenoids, mostly lutein (5.0 mg.g ${ }^{-1}$ dry weight) and $\beta$-carotene $\left(1.0 \mathrm{mg} . \mathrm{g}^{-1}\right)$.

Methodology: We studied the effect of dietary supplementation of a powdered form of $C$. onubensis biomass on growth and health parameters of laboratory rats. Four randomly organized groups of rats $(n=6)$ were fed a standard diet (Diet1, control) or with a diet in which $0.4 \%$ (Diet2), $1.25 \%$ (Diet 3$)$, or $6.25 \%$ (Diet 4$)(w / w)$ of the standard diet weight was substituted with dried microalgal powder, respectively. The four groups of animals were provided ad libitum access to feed for 45 days.

Results: No significant difference was found in growth rates or feed efficiency ratios of the four groups of rats. Histological 
studies of liver and kidney tissue revealed healthy organs in control and microalga-fed animals, while plasma hematological and biochemical parameters were within healthy ranges for all animals. Furthermore, animals fed with diet 4 exhibited a statistically significant decrease of $50 \%$ in both blood VLDL-cholesterol and triglyceride levels.

Conclusions: These data suggest that $C$. onubensis biomass may be useful as a safe food supplement for laboratory animals with an important blood hypolipidemic effect. Furthermore, the microalga also could be used as nutraceuticals source for functional foods.

Aknowledgements: Authors thank the support from Plan Propio de Investigación of the University of Huelva.

Coccomyxa onubensis, rats, safe food, nutraceuticals, hypolipidemic induction

\section{P2-03}

EFFECT OF VIRGIN OLIVE OIL ON PLASMA HOMOCYSTEINE LEVELS IN APOLIPOPROTEIN E (APOE -/-) DEFICIENT MICE

José Manuel Lou-Bonafonte ${ }^{(1)}$, José Miguel Arbonés-Mainar ${ }^{(2)}$, María Angeles Navarro ${ }^{(3)}$, Jesús Osada ${ }^{(4)}$

(1) Dpto. Farmacología y Fisiología (Universidad de Zaragoza); IISA, IA2, CIBER-OBN (ISCIII), Zaragoza, Spain; ${ }^{(2)}$ IACS, IISA, CIBER-OBN (ISCIII), Zaragoza, Spain; (3) Dpto. Bioquímica y Biología Molecular (Universidad de Zaragoza); IACS, IISA, I IA2, CIBER-OBN (ISCIII), Zaragoza, Spain; (4) Dpto. Bioquímica y Biología Molecular (Universidad de Zaragoza); IISA, I IA2, CIBER-OBN (ISCIII), Zaragoza, Spain

Previous results showed that Extra Virgin Olive Oil (EVOO), especially varieties rich in polyphenols, increases betainehomocysteine-methyl-transferase -an enzyme involved in the hepatic metabolism of homocysteine- in the liver of apoE -/- mice. Homocysteine has been considered a very important risk factor for cardiovascular disease that could be influenced by different olive oils.

To this end, we have tested the effect of two types of EVOO (Picual and Arbequina) in order to study plasma homocysteine levels in apoE -/- mice, a well characterized animal model that develops atherosclerosis spontaneously, similar in characteristics to those found in humans. Three groups of mice were fed a standard diet with $0.15 \%$ cholesterol supplemented with either $20 \%$ palm oil (control; $n=11), 20 \%$ VOO Arbequina $(25 \mathrm{mg} / \mathrm{kg}$ of polyphenols; $n=11)$, or VOO $20 \%$ Picual $(90 \mathrm{mg} / \mathrm{kg}$ of polyphenols; $n=10$ ). At the end of the 10 th week of the intervention period, the overnight-fasted mice were sacrificed by $\mathrm{CO}_{2}$ suffocation. Blood samples were collected by cardiac puncture and the plasma was stored at $-80^{\circ} \mathrm{C}$ until further analysis. Thiols (cysteine, glutathione and homocysteine) were analyzed in plasma samples by HPLC.

The results showed: 1) Picual EVOO significantly decreased plasma homocysteine compared to the control and Arbequina groups; 2) Picual EVOO significantly increased the plasma concentration of glutathione compared to the control group; 3) Arbequina EVOO showed no effect on plasma homocysteine concentration with respect to the control group, but increased the concentration of plasma glutathione, although this increase was not statistically significant compared to the control group.

In conclusion: 1) Consumption of Picual EVOO could be especially beneficial for patients with cardiovascular risk factors; 2) These preliminary data in mice warrant further investigation regarding the effects of "picual EVOO" on plasma homocysteine levels in hyperhomocysteinemic patients.

Apolipoprotein E, Knockout mice, Homocysteinemia, Olive oil, Atherosclerosis.

P2-04

MODIFYING EATING HABITS AFTER AN EDUCATIONAL INTERVENTION IN UNIVERSITY WOMEN: A PILOT STUDY

Roberto Ramírez-Domínguez ${ }^{(1)}$, Isabel Bayona ${ }^{(1)}$, Teresa Mingo ${ }^{(1)}$, Mariángeles Ferrer ${ }^{(1)}$, Enrique Márquez ${ }^{(2)}$, Francisco Navas (1) Lucía Pérez-Gallardo ${ }^{(1)}$

(1) UVA, Soria, Spain; ${ }^{(2)}$ CAEP Soria, Soria, Spain

Objective: To assess the impact of an educational intervention in undergraduate women about changes in dietary habits, anthropometric parameters and body composition.

Subject and Methods: Seventeen women aged between 19 and 23 years old have been involved in the intervention program (6 months length). Both at the start (November) and at the end of program (May) anthropometric and body composition data was obtained by bioimpedance. In the same periods, food consumption data was also collected for seven days and were transformed into energy and nutrient intake (Nutriber program). Statistical analysis was carried out by SPSS.22 program, considering statistically significant differences at $p<0.05$.

Results: Following the educational intervention we did not observe any changes in anthropometric parameters or body composition. An increase in the frequency of consumption of fruits, vegetables and fish and in intake of vitamin B3, vitamin $\mathrm{C}$ and folic acid was observed. The intake of total energy (ET) / day and the \% ET from fat was lower after the intervention. Results also revealed an increase in the $\%$ ET provided by proteins $(p=0.009)$ and a tendency to reduce sodium intake $(p=0.054)$.

Conclusions: The implementation of this program of nutritional education contributed to partially improve the eating habits of the participants.

Educational intervention, University students, Women, Food Habit, Dietary Modifications

\section{P2-05}

\section{RELATIONSHIP BETWEEN MATERNAL OBESITY AND} FATTY ACID PROFILE OF BREAST MILK

Sara García ${ }^{(1)}$, Nieves Marta Díaz ${ }^{(2)}$, Maria Virginia Martín ${ }^{(3)}$, Roberto Dorta ${ }^{(4)}$, Nieves Guadalupe Acosta ${ }^{(1)}$, Mercedes Murray (5), Inmaculada González ${ }^{(6)}$, Diana Escuder ${ }^{(7)}$, Covadonga Rodríguez $^{(1)}$ 
(1) Facultad de Ciencias. Universidad de La Laguna, La Laguna, Spain; (2) Facultad de Ciencias de La Salud. Universidad de La Laguna, La Laguna, Spain; (3) Centro Oceanográfico de Las Islas Canarias, Santa Cruz de Tenerife, Spain; ${ }^{(4)}$ Departamento de Estadística I. O. y C. Universidad de La Laguna., La Laguna, Spain; ${ }^{(5)}$ Hospital Universitario de Canarias, La Laguna, Spain; ${ }^{(6)}$ Hospital Universitario de La Candelaria, Santa Cruz de Tenerife, Spain; ${ }^{(7)}$ Hospital 12 de Octubre, Madrid, Spain

Introduction: Pregnancy is characterized by changes in lipid profiles, and an increase of inflammatory response and oxidative stress. In case of maternal obesity (MO) these processes would be aggravated due to increment of lipids, considered harmful for pregnancy development.

Breast feeding is believed a potential factor against obesity. n-3 and n-6 LCPUFA (long chain polyunsaturated fatty acid) are essential fatty acids in animals. Certain n-6 LCPUFA (20:4n-6; ARA) serve as precursors of eicosanoid synthesis as $\mathrm{PGE}_{2}$ (Prostaglandine $\mathrm{E}_{2}$ ), which is involved into adipocytes differentiation and obesity. However eicosanoids derived from eicosapentaenoic acid (EPA; 20:5n-3), such as $\mathrm{LTE}_{4}$ (Leukotriene $\mathrm{E}_{4}$ ), can play a role against obesity. Metabolic changes promoted by obesity can also modify biochemical parameters of breast milk (BM), which composition is affected by maternal diet. In this sense, the fatty acid profile and the eicosanoid levels of BM, could give us as a clue of maternal nutritional status and its relationship with MO condition.

Therefore it is hypothesized that maternal n-3 LCPUFA intake determines the nutritional status in these LCPUFAs, and eicosanoids derived, in BM in relation with $\mathrm{MO}$.

Objective: To study the relationship between maternal obesity and levels of LCPUFAs and eicosanoids in breast milk

Methodology: Lipid composition from BM of 86 normal weight mothers (NWM) and 46 overweight and obese mothers (OWOM) were analyzed. Total protein (TP), lipid class (LC), fatty acid profiles, lipid peroxidation index $(\mathrm{PI})$ and eicosanoids $\left(\mathrm{LTE}_{4}\right.$ and $\mathrm{PGE}_{2}$ ) were determined.

Results: Not differences in total lipid content, TP, LC, and PI were found between groups. BM of NWM showed significantly higher levels of C18:0, C20:0, 20:5n-3 and 22:6 n-3, and total content of n-3-LCPUFA compared to OWOM. Therefore, the ratios n-3/n-6, EPA/AA and DHA/AA were also higher in the BM of NWM. In contrast, C16:1 (n-7), C16:1 (n-9), n-6 LCPUFA such as 20:2 (n-6), 20:3 (n-6), 22:4 (n-6), and total amount of n-6 LCPUFA were significantly higher in BM of OWOM.

$\mathrm{LTE}_{4}$ was in a higher level in BM of NWM, whereas $\mathrm{PGE}_{2}$ level did not shown significant differences between groups. There was a significant negative correlation between $\mathrm{LTE}_{4}$ level and body mass index.

Conclusion: The higher contents of n-3 LCPUFA found in $\mathrm{BM}$ of NWM, as well as $\mathrm{LTE}_{4}$ levels, in contrast with higher levels of n-6 LCPUFA in BM of OWOM, may indicate a differential dietary and metabolic pattern between both groups.

Breast milk, fatty acids, eicosanoids, maternal obesity
P2-06

IN COMPARISON WITH PALM OIL, DIETARY SKIN WALNUT SUPPLEMENTATION DELAYS PROGRESSION OF ATHEROSCLEROSIS LESION IN APOE-DEFICIENT MICE

Sonia Gascón (1), Luis Vicente Herrera (2), Cristina Barranquero (3), Roberto Martínez-Beamonte (4), Joaquin Surra ${ }^{(5)}$, Carmen Arnal (6), Jesus De La Osada Garcia ${ }^{(5)}$, $\mathrm{M}^{\mathrm{a}}$ Angeles Navarro ${ }^{(5)}$

(1) Dpto Fisiología y Farmacología. Fac Veterinaria. Universidad de Zaragoza. CiberObn., Zaragoza, Spain; (2) Dpto. Bioquímica y Biología Molecular y Celular. Fac Veterinaria. Universidad de Zaragoza., Zaragoza, Spain; (3) Dpto. Bioquímica y Biología Molecular y Celular. Fac Veterinaria. Universidad de Zaragoza. CiberObn., Zaragoza, Spain; ${ }^{(4)}$ Dpto. Bioquímica y Biología Molecular y Celular. Fac Veterinaria. Universidad de Zaragoza. CiberObn, Zaragoza, Spain; ${ }^{(5)}$ Dpto. Bioquímica y Biología Molecular y Celular. Fac Veterinaria. Universidad de Zaragoza. CiberObn. Instituto de Investigación Sanitaria de Aragón, Zaragoza, Spain; (6) Dpto. de Patología Animal. Fac Veterinaria. Universidad de Zaragoza. CiberObn. Instituto de Investigación Sanitaria de Aragón, Zaragoza, Spain

Background and objective: Epidemiological studies have demonstrated benefits of nut consumption in a healthy pattern on cardiovascular risk factors and coronary heart diseases, attributed to their fatty acid profile, rich in unsaturated fatty acids, and also to other nutrients. The nut components contribution in the development of atherosclerosis remains incomplete.

Design and Methods: The effect of walnuts on the atherosclerotic lesion was studied in female and male Apoe-knockout mice fed a diet supplemented with $3 \%(w / w)$ walnut and compared with mice receiving an isocaloric diet of similar fat content provided as palm oil, a third group of mice receiving only the walnut skin to evaluated its antioxidants. After 11 weeks, plasma lipid parameters, Pcyox 1 and paraoxonase family hepatic mRNA and aortic en face lesions were measured.

Results: Males receiving walnut and skin walnut diets had lower plasma triglycerides than palm oil group, and both sex groups receiving walnuts and skin walnut diets had lower plasma HDL cholesterol than mice receiving the palm oil diet. Hepatic Pon 2 mRNA increased in skin walnut containing diet compared with palm oil group in both sexes, and no change was observed in Pcyoxl expression. Females consuming the walnut and skin walnut diets showed lower hepatic Pon 3 mRNA than females consuming palm oil, and a decrease of hepatic Ponl mRNA was observed in males supplemented with walnut compared with palm oil group. Males and females consuming the skin walnut diet showed smaller aortic lesion areas than those consuming palm oil. 
Conclusions: Our results suggest that the antiatherosclerotic effect of skin walnut intake in Apoe-deficient mice may be attributed to higher hepatic mRNA Pon2 expression

Walnuts, ApoE-deficient mice, Atherosclerosis, Lipoproteins

P2-07

IN VITRO ANTIDIABETIC POTENTIAL OF COMMERCIAL JUICES WITH HIGH POLYPHENOL CONTENT

Francisco Les ${ }^{(1)}$, Guillermo Cásedas ${ }^{(1)}$, Marta Sofía Valero ${ }^{(1)}$, Jose Miguel Arbonés-Mainar ${ }^{(2)}$, Maria Pilar Gómez-Serranillos ${ }^{(3)}$, Carine Smith ${ }^{(4)}$, Víctor López ${ }^{(1)}$

(1) Department of Pharmacy, Faculty of Health Sciences, Universidad San Jorge, Villanueva de Gállego (Zaragoza), Spain; (2) Adipocyte and Fat Biology Laboratory (AdipoFat), Translational Research Unit, University Hospital Miguel Servet, Instituto Aragonés de Ciencias de la Salud (IACS), Instituto de Investigación Sanitaria Aragón (IIS Aragón), Zaragoza, Spain; ${ }^{(3)}$ Department of Pharmacology, Faculty of Pharmacy, Universidad Complutense de Madrid, Madrid, Spain; ${ }^{(4)}$ Department of Physiological Sciences, Stellenbosch University, Stellenbosch, South Africa

WHO estimates that diabetes will be the seventh cause of death by 2030 and in most cases type 2 diabetes can be prevented modifying lifestyle (physical activity and diet). In recent years, numerous studies have shown the beneficial effects of polyphenols and its protective effects on diabetes and the metabolic syndrome. American cranberry or "cranberry" (Vaccinium macrocarpon), blueberry or bilberry (Vaccinium myrtillus), cherry (Prunus cerasus) and pomegranate (Punica granatum) are fruits with an interesting polyphenol content. That is why they have been selected to perform this preliminary study in terms of its antioxidant properties and effects on glycaemic control-related enzymes such as $\alpha$ glucosidase and dipeptidyl peptidase 4.

Juices selected for this study (Rabenhorst ${ }^{\mathbb{R}}$ ) were lyophilized and analyzed by HPLC-DAD. The Folin-Ciocalteu method was used for the determination of polyphenols. Effects on $\alpha$-glucosidase and dipeptidyl peptidase 4 were measured by enzyme inhibition tests using colorimetric and fluorimetric assays. The antioxidant activity was measured as scavenging properties of superoxide radical generated by xanthine oxidase system. Data analysis was performed using GraphPad Prism.

The juices showed a significant but a variable content of polyphenols, being blueberry (Vaccinium myrtillus) and pomegranate (Punica granatum) the greatest in terms of polyphenolic composition and antioxidant capacity against superoxide radicals. They also showed inhibitory effects on the tested enzymes, but in no case greater than acarbose or sitagliptin, used as reference drugs. The results seem to indicate these juices might be of nutritional interest to help in the glycemic control and diabetes prevention.

Natur Import is acknowledged for providing juices for this study as well as CITA Aragón for lyophilization

Pomegranate, blueberry, antioxidant, diabetes
P2-08

EFFECT OF CONSUMPTION OF A LYCOPENE-ENRICHED COMMERCIAL PRODUCT OF ORGANIC EXTRA VIRGIN OLIVE OIL (ACEITEROL ${ }^{\circledR}$ ) ON PROSTATE HEALTH Cristina Carrasco
Beatriz Rodríguez $^{(1)}$, Luis Blanco

(1) University of Extremadura, Badajoz, Spain; (2) Dieta Mediterránea Aceites y Vinagres S.A., Zafra (Badajoz), Spain;

(3) Urology Service of Hospital Infanta Cristina, Badajoz, Spain

Introduction: Lycopene is the most abundant dietary carotenoid, appearing in high concentrations in foods such as tomato (Lycopersicum spp.) and derived products (sauce, ketchup, juice, etc.). Epidemiological evidence shows that its consumption would be effective both in the prevention and therapy of various diseases, such as prostate cancer. Despite of be a compound widely accepted in the field of food industry due to these healthy properties, the influence on some of the most basic aspects of human health remains unknown.

Objectives: The aim of this study was to determine the effect of consumption of a lycopene-enriched commercial product of organic extra virgin olive oil (Aceiterol ${ }^{\circledR}$ ) on sleeping quality and prostate, emotional and dermal health of men.

Material and Methods: The study was carried out on a total of 20 men, all over the age of 50 . Volunteers were divided into control group ( $n=10$, without prostate pathologies) and pathological group ( $n=10$, diagnosed with prostate adenoma). Participants were assessed initially for obtaining their baseline values of sleeping quality, prostate symptoms (International Prostate Specific Scale or IPSS), prostate specific antigen (PSA) and protein C reactive (PCR) serum levels, emotional health (Test State AnxietyTrait or STAI) and dermal health (hydration, sebum, elasticity, melanin and erythema). Then, participants consumed the product Aceiterol $^{\circledR}(20 \mathrm{ml} ; 0.4 \mathrm{mg} / \mathrm{ml}$ of LYC) daily, at breakfast or lunch, for 30 days (month treatment). Beyond the month treatment, participants were assessed again attending the above specified parameters.

Results: This preliminary study showed that Aceiterol ${ }^{\circledR}$ consumption induced a slight positive tendency in all parameters studied. More remarkable results were those related to PSA serum levels and scores of IPSS and STAI tests of pathological group, as well as sleeping quality (in terms of nocturnal activity) of both groups. Additionally, dermal health (particularly, the apparition of erythema) was significantly reduced in control group after treatment.

Conclusions: Aceiterol ${ }^{\circledR}$ consumption during 1 month seems to positively influence sleeping quality, prostate symptoms, PSA and PCR serum levels and emotional and dermal health, probably due to the diversity of antioxidant and anti-inflammatory components, particularly lycopene, contained in the food matrix of extra virgin olive oil.

Acknowledgments: Supported by Junta de Extremadura-Fondo Social Europeo de Desarrollo Regional (FEDER) grant BBB021GR15051 and by research agreement between University of Extremadura and Dieta Mediterránea Aceites y Vinagres S.A. (Ref. 142/15).

Extra virgin olive oil, lycopene, prostate adenoma 


\section{ASSESSMENT OF DIETARY HABITS AND NUTRITIONAL STATUS IN UNIVERSITY STUDENTS}

María Daniela García, Cristina Carrasco, María Garrido, Ana Beatriz Rodríguez

University of Extremadura, Badajoz, Spain

Inadequate eating behaviors such as high-fat or low-nutrient diets are growing, particularly between young people. These unbalanced eating habits may lead to the development of future health-related diseases. The aim of this study was to evaluate the dietary habits and nutritional status in university students in order to determine excesses and/or deficiencies in their diet according recommended dietary allowances. The study was conducted on 52 students (19-21 years-old) who belong to the Faculty of Science at the University of Extremadura (Badajoz, Spain). All the participants collected all the information about food and beverage consumption during 5 days. The nutritional evaluation was carried out using the DIAL $^{\circledR}$ software (Alce Ingenieria, Madrid). Descriptive statistics were used to describe the features of the data. The results showed that the students had a normal body mass index (BMI), correct water consumption and minimal distilled alcohol consumption. However, an excessive caloric intake provided by proteins and fat was found. Moreover, an inadequate carbohydrate intake was detected. Related to vitamins and minerals, deficiencies were found in vitamins A, $\mathrm{D}, \mathrm{E}$, and folic acid, as well as potassium, magnesium and iron. Lipid profile showed an excessive ingestion of saturated fatty acids, whereas the monounsaturated and polyunsaturated fatty acids remained under recommended values. Graphic representation of calorie intake by food groups showed that cereals, followed by meat and dairy products, were the most consumed between university students. On the contrary, sauces, condiments, snacks and eggs were the least represented. Moreover, the nutritional analysis of the results revealed a low presence of fruit, vegetables and fish in the diet of participants. Additionally, calorie intake representation along the day showed that the percentage of consumed calories was higher at lunch, dinner and breakfast compared to the calories supplied by the mid-morning snack as well as late-night snack. In conclusion, the nutritional status of the population evaluated might be improved. In this regard, user-friendly nutritional tools such as DIAL software in degrees and masters of Health Sciences (Biology, Biotechnology and Medicine), might be useful to make young people (and future professionals) aware of need to change health habits in the prevention of most prevalent diseases.

Acknowledgments: Supported by Junta de Extremadura-Fondo Social Europeo de Desarrollo Regional (FEDER) grant BBB021-GR15051. Maria Garrido holds a research postdoctoral fellowship from Gobierno de Extremadura [jointly financed by the European Regional Development Fund (ERDF); ref. PO14013].
EVALUATION OF THE ANTIOXIDANT ACTIVITY OF TWO ARAGON EDIBLE FLOWERS (BORAGO OFFICINALIS AND ALLIUM CEPA) IN THE MODEL ORGAMISM CAENORHABDITIS ELEGANS

Cristina Moliner, Inés Reigada, Elisa Langa, Marta Sofia Valero, Carlota Gómez-Rincón

Universidad San Jorge, Villanueva de Gállego, Spain

Aging and age-associated diseases, such as diabetes, atherosclerosis or neurodegeneration disease, has long been associated with oxidative stress. Some time ago is beeing paid attention the use of food and plants as natural antioxidants.

Edible flowers have always been part of the human diet, but recently their use has been increased, not only for their culinary properties but also due to their antioxidant capacity, considering them nutraceuticals, but most of the time their properties still officinalis (borage flowers) and Alliun cepa (onion flowers). Both of them grown in Aragon, Spain.

The objetive of this research was to establish the capacity of the ethanolic extract of two Aragon edible flowers: Borago officinalis and Alliun cepa to improve the response in oxidative stress induced by juglone in C. elegans.

Both extracts were prepared from flowers by percolation using ethanol as solvent. The antioxidant activity was evaluated in vitro using DPPH photometric assay[1] in a range of concentrations between 2,5 and $250 \mathrm{mg} / \mathrm{mL}$ and in vivo measuring the response to reduce oxidative stress induced by a lethal dose of juglone[2] $(150 \mu \mathrm{M})$ in C. elegans pretreated with the extracts at doses ranging beetween 50 and $500 \mathrm{mg} / \mathrm{mL}$.

Neither of the two extracts had reached the $\mathrm{IC}_{50}$ (concentration of extract required to scavenge $50 \%$ of DPPH radicals). Studied populations of $C$. elegans significantly increased the rate of survival when treated with both extracts when compared to control. A greater protection against oxidative stress induced by juglone was provided by onion flowers (increased of around $15,6 \%$ in the survival rate) than by borage flowers $(11,40 \%)$.

The results support the potencial use of flowers from B. officinalus and A. cepa as nutracetics due to its antioxidant properties.

[1] López, Akerreta, Casanova, García-Mina, Cavero, Calvo. In vitro antioxidant and anti-rhizopus activities of Lamiaceae herbal extracts. Plant Foods for Human Nutrition : 2007: 62(4): 151-155.

[2] Surco-Laos, Cabello, Gómez-Orte, et all. Effects of O- methylated metabolites of quercetin on oxidative stress, thermotolerance, lifespan and bioavailability on Caenorhabditis elegans. Food \& Function 2011, 2, 445-456.

Onion, borage, nutraceutical, model organism, DPPH 
ANTIOXIDANT CAPACITY IN VITRO AND IN VIVO OF TWO MEDICINAL PLANTS MINT (MENTHA PIPERITA) AND FENNEL (FOENICULUM VULGARE)

Inés Reigada, Cristina Moliner, Carlota Gómez-Rincón, Marta Sofía Valero, Elisa Langa

Universidad San Jorge, Villanueva de Gállego, Spain

The rising popularity of phytoterapic remedies makes necessary to prove scientifically the therapeutically proprieties granted to the most used plants. Antioxidant consumption has been associated with several positive effects on the organism. It has been postulated that antioxidant substances protect our cells from oxidative stress, slowing ageing. On the other hand oxidative stress is considered an influencing factor on pathological process such as cardiovascular, inflammatory, cancer and neurodegenerative. Because of these reasons there is an increase of medicinal plants consumption. Two of these popular plants are mint (Mentha piperita) and fennel (Foeniculum vulgare).

The aim of this study is to check the antioxidant capacity of these two plants, both in vitro and in vivo.

To do this, an ethanolic soxhlet extraction of the dried plant drugs was carried out. The antioxidant potential of that extract was tested both in vitro (by the DPPH Photometric Assay[i]) and in vivo (through an assessment of Caenorhabditis elegans resistance to oxidative stress induced by juglone [ii]).

At DPPH method Mint had an $\mathrm{IC}_{50}$ of $34.574 \mu \mathrm{g} / \mathrm{mL}$ while fennel showed an $\mathrm{IC}_{50}$ of $259.685 \mu \mathrm{g} / \mathrm{mL}$. In vivo essay worms pretreated with fennel at a concentration of $500 \mu \mathrm{g} / \mathrm{mL}$ and $250 \mu \mathrm{g} / \mathrm{mL}$ showed an increase of the survival of $32.01 \pm 0.31 \%$ and 12.62 $\pm 0.13 \%$ respectively. Mint didn't show differences with the control.

In conclusion it could be said that there is not a relevant correlation between the results obtained by DPPH method and the ones obtained in the in vivo assay. Mint is a better antioxidant at the in vitro method, but fennel proves to be a better antioxidant in vivo.

[i] López V, Akerreta S, Casanova E, García-Mina JM, Cavero RY, Calvo MI, 2007. In vitro antioxidant and anti-rhizopus activities of Lamiaceae herbal extracts. Plant Foods for Human Nutrition 62(4): 151-155.

[ii] Kampkötter, A.; Timpel, C.; Zurawski, R. F.; Ruhl S.; Chovolou, Y.; Proksch, P.; Wätjen, W. Increase of stress resistence and lifespan of Caenorhabditis elegans . Comparative Biochemistry and Physiology Part B 149 (2008), 314-323.

DPPH method, Caenorhabditis elegans, Mentha piperita, Foeniculum vulgare

\section{SOLUBLE ENDOGLIN MODIFIES THE INFLAMMATORY RESPONSE IN MICE}

Laura Ruiz Remolina, Claudia Ollauri Ibáñez, Lucia Pérez Roque, Elena Núñez Gómez, Cristina Egido Turrión, Fernando Pérez Barriocanal, José Miguel López Novoa, Miguel Pericacho Bustos, Alicia Rodríguez Barbero

Departamento de Fisiología y Farmacología-USAL, Universidad de Salamanca, Spain

The acute inflammatory response is characterized by three main vascular effects: vasodilation with increased blood flow, higher vascular permeability, and leukocytosis into the injured tissues. Inflammation is associated with the tissue repair process, in which our group has shown that endoglin plays an important role. Endoglin (CD105), a transforming growth factor beta $(\mathrm{TGF} \beta)$ co-receptor, is related with vascular and angiogenic diseases. The soluble form of endoglin (sEng) has been less studied. Elevated levels of sEng have been found in women with preeclampsia and patients with cancer or myocardial infarction, all of them with associate inflammation. sEng has been proposed as a marker of preeclampsia and cancer, however, the role of endoglin in these diseases remains unknown. We hypothesize that sEng play a role in the inflammatory process associated with the start of this diseases. To test the hypothesis, we generated an animal model with elevated circulating levels of human sEng. We induced in these animals an acute inflammation through two models: 1) LPSinduced acute lung injury and 2) carrageenan-induced air pouch. Recruitment of leukocytes was measured in the air pouch lavage and in the bronchoalveolar lavage fluid (BALF). In both cases we found a reduction in the leukocyte extravasation in sEng compared with the WT. Leukocyte infiltration was analyzed by histological examination of LPS-treated lungs from sEng and WT mice. We found again, lower leukocyte infiltration in the sEng LPS-treated lungs than in WT lungs. In order to investigate whether the decrease of leukocyte infiltration and extravasation induced by circulating sEng is mediated by changes in vascular permeability, we measured the amount of proteins and FITC-Dextran in the BALF. We found a correlation between the decrease in leucocyte infiltration and the reduction in permeability in the sEng mice respect to the WT. Finally, we analyze whether circulating sEng was involved in the release of inflammatory cytokines in BALF and lung tissue from LPS-treated mice. The concentration of TNF $\alpha$, IL $1 \mathrm{~b}$ and IL6 were significantly lower in the samples from sEng mice respect the WT.

Thus, circulating sEng plays an active role in the inflammatory response, modifying key processes of inflammation such as leukocyte recruitment to the site of inflammation, vascular permeability and release of inflammatory cytokines. These results open a new therapeutic target for the treatment of diseases with an inflammatory component in the initial state where early intervention is essential to the management of the disease.

Inflammation, soluble endoglin, leukocyte recruitment, vascular permeability, cytokines, mouse 
P2-13

AGING EFFECTS ON CAROTID BODY AND VASCULAR RESPONSES FROM RATS EXPOSED TO CHRONIC INTERMITTENT HYPOXIA

Elena Olea ${ }^{(1)}$ Inmaculada Docio ${ }^{(1)}$, Teresa Gallego-Martín ${ }^{(1)}$, Ana Obeso (1), Teresa Agapito (1), Ricardo Rigual (1), Angela Gomez-Niño ${ }^{(2)}$, Asunción Rocher ${ }^{(1)}$

(1) Dpto. Bioquímica y Biología Molecular y Fisiología, IBGM. Universidad de Valladolid/CSIC. CIBERES, ISCiii, Valladolid, Spain; ${ }^{(2)}$ Dpto. Biología Celular, Histología y Farmacología, IBGM. Universidad de Valladolid/CSIC. CIBERES, ISCiii, Valladolid, Spain

Chronic Intermittent Hypoxia $(\mathrm{CIH})$ is considered one of the main causes of cardiovascular and metabolic alterations observed in the obstructive sleep apnea syndrome. Repetitive episodes of hypoxia/ re-oxygenation produce oxidative stress and inflammation that could predispose to cumulative injury and acceleration of the aging process. Previous observations show that deleterious effects of $\mathrm{CIH}$ seem to be less pronounced in aged animals. It has also been reported that $\mathrm{CIH}$ produces sympathetic hyperactivity, endothelial dysfunction and systemic hypertension. Carotid body (CB) sensitization by recurrent hypoxic stimulation has been proposed as the origin of these alterations.

The aim of this study is to compare vascular and CB responses and hypertensive changes in young and aged animals exposed to $\mathrm{CIH}$, relating age and $\mathrm{CIH}$ induced effects. Four groups of male Wistar rats were used: young ( 3 months; $n=32$ ) and aged (18 months; $n=32)$ in normoxia (C 3/18 months; $n=16)$ and exposed to ( $21 \%$ $\mathrm{O}_{2}-80 \mathrm{~s} / 5 \% \mathrm{O}_{2}-40 \mathrm{~s} 8 \mathrm{~h} /$ day; 14 days) CIH (3/18 months; $\left.n=16\right)$. Ventilatory responsiveness to hypoxic and hypercapnic test was measured by whole body plethysmography. Aged rats ventilated less than younger under any conditions. On animals anaesthetized with ketamine $(100 \mathrm{mg} / \mathrm{Kg}$ ) and diazepam ( $5 \mathrm{mg} / \mathrm{Kg}$; ip.), blood $\mathrm{pO}_{2}, \mathrm{pCO}_{2}$ and $\mathrm{SatO}_{2}$ were analyzed by gasometry at different inspired air $\mathrm{O}_{2}$ percentages (5-21\%). In old control and $\mathrm{CIH}$ rats, $\mathrm{SatO}_{2}$ at $\mathrm{PO}_{2} \leq 50 \mathrm{mmHg}$ was lower than in young control rats. Mean arterial blood pressure (MABP) and pulse pressure were higher in aged than young control rats but got similar values after $\mathrm{CIH}$. Vascular contractile responses were studied by wire myography. Using external carotid arteries, wall tension generated in response to phenylephrine $(0.01$ to $3 \mu \mathrm{M})$ was higher in aged and $\mathrm{CIH}$ than young control rat arteries. Carbachol $(10 \mu \mathrm{M})$ dependent relaxations were greater in young control than in aged and $\mathrm{CIH}$ rat arteries. Larger MABP and vascular properties alterations in aged animals could explain the lack of MABP increase observed in old rats after $\mathrm{CIH}$. Conversely, $\mathrm{CIH}$ enhanced the $\mathrm{CB}$ secretory response to hypoxia $\left(5 \% \mathrm{O}_{2}\right)$ in young and aged rats ( 250 vs. $300 \%$ ), correlating with $\mathrm{Ca}_{\mathrm{i}}{ }^{2+}$ increases in freshly dissociated chemoreceptor cells. Mechanisms underlying the enhanced $\mathrm{CB}$ reactivity to hypoxia induced by $\mathrm{CIH}$ could be due to a prooxidative status, as observed in other tissues.

Supported by grants: MINECO BFU2015-70616R; ISCiii CIBER CB06/06/0050

Intermittent hypoxia; carotid body; aging; catecholamines; ventilation
P2-14

\section{POSITIVE BIOCOMPATIBILITY OF NANOCRYSTALLINE} GLASS-LIKE CARBON THIN FILMS

Noela Rodriguez-Losada ${ }^{(1)}$, Pablo Romero ${ }^{(2)}$, Roberto Guzman De Villoria $^{(2)}$, Jose A. Aguirre ${ }^{(1)}$

(1) Departamento de Fisiologia. Facultad de Medicina. Campus de Teatinos, 29010 Malaga, Spain; ${ }^{(2)}$ IMDEA Materials Institut, C/ Eric Kandel 2, 28906 Getafe, Madrid, Spain

The interest in carbon nanomaterials with high transparency and electrical conductivity has grown within the last decade in view of a wide variety of applications, including biocompatible sensors, diagnostic devices and bioelectronic implants. The aim of this work is to test the biocompatibility of particular nanometer-thin nanocrystalline glass-like carbon films (NGLC), a disordered structure of graphene flakes joined by carbon matrix (Romero et al., 2016). We used the SN4741 cell line from substantia nigra dopaminergic cells derived from transgenic mouse embryo cells (Son et al., 1999). Some cells were cultured on top of NGLC films $(5,20$ and $80 \mathrm{~nm})$ and other with NGLC nanoflakes (approx. 5$10 \mu \mathrm{m}^{2}$ ) in concentrations: $1,5,10,20$ and $50 \mu \mathrm{g} / \mathrm{ml}$, during $24 \mathrm{~h}$, 3 days and 7 days. Cells growing in normal conditions were defined under culture with DMEM supplemented with $10 \%$ FCS, Glucose $(0,6 \%)$, penicillin-streptomycin $(50 \mathrm{U} / \mathrm{ml})$ and Lglutamine $(2 \mathrm{mM})$ at $5 \% \mathrm{CO} 2$ humidified atmosphere. Nanoflakes were resuspended in DMEM at the stock concentration (2 g/l). The experiments were conducted in 96 well plates (Corning) using 2500 cells per well. For MTT analysis, the manufacturer recommendations were followed (Roche, MTT kit assay): positive control with a $10 \%$ Triton X-100 treatments $(15 \mathrm{~min})$ and negative control without neither Triton X-100 nor NGLC. As apoptosis/ necrosis assay we used LIVE/DEAD ${ }^{\circledR}$ Viability/Cytotoxicity Assay Kit (Invitrogen). In a separate experiment, cells were cultured on top of the NGLC films for 7 days. Primary antibodies: antisynaptophysin (SYP, clone SY38, Chemicon) and goat antiGIRK2 (G-protein-regulated inward-rectifier potassium channel 2 protein) (Abcom) following immunofluorescence protocol. WB for proteins detection performed with a polyclonal antirabbit proliferating cell nuclear antigen (PCNA). Results demonstrated the biocompatibility with different concentration of NGLC varying the degree of survival from a low concentration $(1 \mu \mathrm{g} / \mathrm{ml})$ in the first $24 \mathrm{~h}$ to high concentrations $(20-50 \mu \mathrm{g} / \mathrm{ml})$ after 7 days as it is corroborated by the PCNA analysis. Cells cultured on top of the film showed after 7 days axonal-like alignment and edge orientation as well as net-like images. Neuronal functionality was demonstrated to a certain extent through the analysis of coexistence between SYP and GIRK2. In conclusion, this nanomaterial could offer a powerful platform for biomedical applications such as neural tissue engineering. Supported by UMA, Campus Excelencia Internacional Andalucia Tech, Spain. We thank Prof. Arenas, Karolinska Institutet, Stockholm, Sweden, for SN4741 cell line. R.G.V. acknowledges Spanish Ministry of Science and Innovation for funding through a Ramon y Cajal Fellowship.

Glass-like carbon, SN4741 cell line, biocompatibility, neuronal differentiation 
P2-15

STATIC MAGNETIC FIELDS ON VISUAL AND SOMATOSENSORY CORTEX: EFFECT ON SPONTANEOUS AND EVOKED ACTIVITY IN ANESTHETIZED RAT AND MONKEY

Casto Rivadulla ${ }^{(1)}$, Jordi Aguilá ${ }^{(1)}$, Sandra Prieto ${ }^{(1)}$, Juan Aguilar (2) Javier Cudeiro ${ }^{(1)}$

(1) Neurocom Universidad de A Coruña and INIBIC, A Coruña, Spain; ${ }^{(2)}$ Hospital Nacional de Parapléjicos de Toledo, Toledo, Spain

Static magnetic stimulation (tSMS) has emerged as an affordable and easy to use alternative to well stablished neuromodulatory techniques (TMS or tDCS) in some procedures. The aim of this study was to analyze the effect of static magnetic stimulation on visual and somatosensory cortex in anaesthetized monkey and rats.

EEG recordings were made in the somatosensory cortex of 6 rats, and electrocorticogram (tungsten electrodes) was recorded in the visual cortex of 1 monkey under sevofluorane anesthesia. tSMS was made through a cylindrical neodymium magnet of $6 \mathrm{~cm}$ radius and 0,5 Tesla.

In control conditions, in rats, the dominant frequencies in the Fast Fourier Transform (FFT) during spontaneous activity were $0.5 \mathrm{~Hz}$, while in monkey FFT has shown two peaks at 2 and $7 \mathrm{~Hz}$. In both cases tSMS tend to potentiate lower frequencies. In rat there was a shift in the dominant peak to $0.4 \mathrm{~Hz}$, and in monkey TMS reduced frequencies around $7 \mathrm{~Hz}$ and increased the power of lower frequencies.

Responses to visual stimulation in monkey visual cortex were reduced during tSMS, however in rat somatosensory cortex, the responses to electrical stimulation $(0.1 \mathrm{~Hz})$ in the hindpaw, varied according to the cortical state.

In control conditions, the amplitude of the evoked potential was higher during up states $(0.21 \mathrm{mV})$ versus down states $(0.12 \mathrm{mV})$, and interestingly, tSMS reduced the amplitude of evoked potentials during up states $(41.26 \%)$ while it increases the responses during the down states $(27.1 \%)$.

Despite this uniform effect on the frequency domain, the results achieved on evoked responses showed a much more complex profile indicating that the sign of the effect is depending on cortical activity.

Supported by: Ministerio de Ciencia e Innovación (Spain), BFU2013-45343-P

Neuromodulation, oscillatory activity, magnetic fields

P2-16;

EFFECT OF EPIGALLOCATECHIN GALLATE OR MELATONIN TREATMENT ON RETINAL MORPHOLOGY IN THE P23H RAT

Lorena Perdices ${ }^{(1)}$, Lorena Fuentes-Broto ${ }^{(2)}$, Laura FernándezSánchez ${ }^{(3)}$, Francisco Segura ${ }^{(1)}$, Ana Isabel Sánchez-Cano ${ }^{(4)}$, Nicolás Cuena ${ }^{(3)}$, Isabel Pinilla ${ }^{(5)}$
(1) Institute for Health Research of Aragón (IIS Aragón), Zaragoza, Spain; ${ }^{(2)}$ Institute for Health Research of Aragón (IIS Aragón); Department of Pharmacology and Physiology, University of Zaragoza, Zaragoza, Spain; ${ }^{(3)}$ Department of Physiology, Genetics and Microbiology, University of Alicante, Alicante, Spain; ${ }^{(4)}$ Department of Applied Phsysics, University of Zaragoza, Zaragoza, Spain; ${ }^{(5)}$ Institute for Health Research of Aragón (IIS Aragón); Department of Surgery, University of Zaragoza; Universitary Hospital Lozano Blesa, Zaragoza, Spain

Retinitis Pigmentosa (RP) constitutes a large heterogeneous group of inherited neurodegenerative retinal disorders that cause a progressive loss of retinal function and represent a major cause of blindness. $\mathrm{P} 23 \mathrm{H}$ is one of the most common mutations in rhodopsin gene, being responsible for one third of RP cases in the USA. This dominant mutation triggers numerous modifications at anatomical level, which are exacerbated by oxidative stress. Several investigations have documented the high antioxidant and neuroprotective properties of epigallocatechin gallate (EGCG) and melatonin. In this study, we evaluate the morphological changes that these natural compounds might produce in the retina of an animal model of RP, the $\mathrm{P} 23 \mathrm{H}$ rat.

Twenty heterozygotes $\mathrm{P} 23 \mathrm{H}$ line 1 rats, offspring of $\mathrm{P} 23 \mathrm{H}$ Sprague-Dawley(SD)-background crossed with Long Evans (LE) rats, were used and compared to twenty SD crossed with $\mathrm{LE}$ rats, the reference group. Vehicle, or $10 \mathrm{mg} / \mathrm{kg} /$ day of Melatonin and/or EGCG were administered in the drinking water from 30 to 180 postnatal days. Animals were euthanized with CO2 at P180 and their eyes were then enucleated, fixed and cut on a cryostat, obtaining sections which were treated for immunostaining. As primary antibodies, retinas were labeled with anti-recoverin and anti-protein-kinase $\mathrm{c} \alpha(\mathrm{PKC} \alpha)$. Recoverin was used to identify all photoreceptor cells, while $\mathrm{PKC} \alpha$ labeled rod bipolar cells, which are the only output cells of rod photoreceptors in the outer plexiform layer (OPL), being important to see whether rod bipolar cells are particularly vulnerable during the course of rod photoreceptor degeneration.

Heterozygous $\mathrm{P} 23 \mathrm{H}$ rats showed a progressive loss of rod photoreceptors over several months compared to reference group's retinas. At the end of the experiment, it is observed signs of degenerative changes in all the layers: cell damage and death and a completely disorganization of the whole retina. $\mathrm{P} 23 \mathrm{H}$ rats treated with antioxidants exhibits a slower degeneration than rats without any treatment.

Our study revealed that oral treatment of melatonin or EGCG delayed retinal degeneration in $\mathrm{P} 23 \mathrm{H}$ rats, stimulating cell surviving.

Funded by Carlos III Health Institute, FIS Grant PI13/01124

Predoctoral Grant of Aragon Government (L. Perdices, F. Segura)

Antioxidants, retinal degeneration, immunohistochemistry

P2-17

POSSIBLE THERAPEUTIC EFFECTS OF MESENCHYMAL STEM CELLS OBTAINED FROM ADIPOSE TISSUE RAT IN PARKINSON MODELS

Mercedes Cano ${ }^{(1)}$, Mario Muñoz-Pinto ${ }^{(2)}$, Leila Berrakad ${ }^{(1)}$, Rafael Medina ${ }^{(1)}$, Antonio Ayala ${ }^{(3)}$ 
(1) Departament of Physisiology, Faculty of Pharmacy, University of Seville, Seville, Spain; ${ }^{(2)}$ Department of Biochemistry, Faculty of Pharmacy, University of Seville., Seville, Spain; ${ }^{(3)}$ Department of Biochemistry, Faculty of Pharmacy, University of Seville, Seville, Spain

The gradual aging is not considered as a physiological disorder. The problem begins when the diseases associated with age increase, like Parkinson's disease (PD). It is a chronic, progressive disorder characterized by continuous and selective loss of dopaminergic neurons located in the substantia nigra pars compacta $(\mathrm{SNpc})$. The stem cells derived from adipose tissue (ADSC) have been proposed as an ideal source of stem cells for regenerative medicine because its numerous advantages, not only for the potential to differentiate into neurons but for their immunomodulatory properties. So, the aim of this work has been to contribute to the possibility that cell therapy with ADSC might be a good strategy to address the PD.

To evaluate the therapeutic potential of the stem ADSC behavioral rotation test and immunohistochemistry asssays were performed in rats with two different models of PD (LPS or 6- OHDA), in which ADSCs were injected as cell therapy and the number of dopaminergic neurons (positive neurons for TH antibody) or the amount of activated microglia (positive for 6-OX antibody) were quantified at 10 days and 4 month after treatment with ADSC.

Stereology revealed that both models of PD, treated with LPS or injured with 6-OHDA and cell therapy, increased TH positive neurons and decreased microglial activation. However, comparing both neurodegenerative models, microglial activation resulting from microinjection of 6-OHDA was less than compared to the model injured with LPS. In turn, ADSC treatment had no significant effect in the case of 6-OHDA microgial activation.

The rotation test revealed that 10 days after animals were treated with ADSC, hardly changes observed in the number of turns relative to untreated animals. However, at 4 months, the number of turns decreases to resemble the control group.

In conclusion, we show that ADSCs have a neuroprotective effect on dopaminergic neurons possibly through a antiinflammatory mechanism by modulation of microglial activation in LPS model case. While in the model injured 6-OHDA, it appears that the neuroprotective effect is unrelated to the microglia activation, but could be due to a direct effect on dopaminergic neurons.

Aging, Parkinson disease, Adipose stem cells

P2-18

CALCIUM SIGNAL REMODELLING AND SUSCEPTIBILITY TO CELL DEATH IN AGED HIPPOCAMPAL NEURONS IN LONG-TERM CULTURE

María Calvo-Rodríguez ${ }^{(1)}$, Monica García Durillo ${ }^{(1)}$, Carlos Villalobos $^{(1)}$, Lucia Nuñez ${ }^{(2)}$

(1) Institute of Molecular Biology and Genetics (IBGM), University of Valladolid and Spanish National Research Council (CSIC), Valladolid, Spain; ${ }^{(2)}$ Dept. of Biochemistry and Molecular Biology and Physiology. University of Valladolid, Valladolid, Spain
Cognitive decline and susceptibility to neuron cell death after insult are strongly associated to aging, yet mechanisms of agingdependent neuron dysfunction and death are poorly known. Neuron death after excitotoxicity and Alzheimer's disease (AD) have been related to intracellular $\mathrm{Ca}^{2+}$ dishomeostasis. Accordingly we asked whether $\mathrm{Ca}^{2+}$ homeostasis in neurons is remodelled during aging. For this end, we employed long-term cultures of rat hippocampal neurons, a model of in vitro aging, along with $\mathrm{Ca}^{2+}$ imaging and immunofluorescence. We found that the glutamate receptor agonist NMDA and amylod $\beta$ peptide oligomers $\left(A \beta_{o}\right)$, the most likely neurotoxin in $\mathrm{AD}$, induced apoptotic cell death in long-term cultures of hippocampal neurons (15-20 DIV), corresponding to aged neurons, but not in short-term cultures $(2-7$ DIV), corresponding to young neurons. Interestingly, $\mathrm{Ca}^{2+}$ increases induced by NMDA and $\mathrm{A} \beta_{\mathrm{o}}$ are much larger in aged neurons than in young cultures. Moreover, bioluminescence imaging of mitochondrial $\mathrm{Ca}^{2+}$ concentration in neurons transfected with targeted aequorin showed that NMDA and $A \beta_{0}$ induced mitochondrial $\mathrm{Ca}^{2+}$ uptake in aged neurons but not in young ones. We also tested whether transfer of $\mathrm{Ca}^{2+}$ from the ER to mitochondria is influenced by in vitro aging. We found that aged-neurons show an increase of the filling state of the stores. In addition, $\mathrm{Ca}^{2+}$ responses due to mobilization of $\mathrm{Ca}^{2+}$ stored in the ER, through inositol-1,4,5-triphosphate receptors $\left(\mathrm{IP}_{3} \mathrm{Rs}\right)$ with acetilcholine $(\mathrm{ACh})$ are higher in aged cultured neurons that in young cultures. In addition, $\mathrm{Ca}^{2+}$ release induced by $\mathrm{ACh}$ was taken up by mitochondria in aged neuros but not in young cultures, thus suggesting enhanced transfer of $\mathrm{Ca}^{2+}$ from the ER to mitochondria in aging. Finally, we found that store-operated $\mathrm{Ca}^{2+}$ entry (SOCE), a Ca ${ }^{2+}$ entry pathway required for mushroom spines stability and memory storage, decreases with age in neurons. Qntitative immnufluorescence suggested that the above changes were mediated by age-related changes in NMDA receptor subunit composition, mitochondrial $\mathrm{Ca}^{2+}$ uniporter and molecular players involved in SOCE. Collectively, these data suggest that aging promotes an important remodeling of subcellular $\mathrm{Ca}^{2+}$ homeostasis that may contribute to cognitive decline and susceptibility to neuron damage in the elderly.

This work was supported by grants from Junta de Castilla y León, Spain (VA145U13) and Ministerio de Economía y Competitividad (BFU2012-37146 and BFU2105-70131R), Spain. MCR was supported by Junta de Castilla y León and the European Social Fund.

Calcium, hippocampal neurons, aging

P2-19

RESTRAINT STRESS-INDUCED C-FOS EXPRESSION IN GOLDFISH (CARASSIUS AURATUS) PALLIUM

Rosa María Giraldez-Perez ${ }^{(1)}$, Susana P. Gaytan ${ }^{(1)}$, Sara Uceda (2), Benjamin Rodríguez-Expósito ${ }^{(2)}$, Fernando Rodriguez ${ }^{(2)}$, Rosario Pasaro ${ }^{(1)}$

(1) Dept. Physiology, Faculty of Biology, University of Seville, Seville, Spain; ${ }^{(2)}$ Laboratory of Psychobiology, Faculty of Psychology, University of Seville, Seville, Spain 
c-Fos is an immediate early gene, and is rapidly and transiently induced in neurons of the central nervous system of mammals and fish as a result of neuronal stimulation. The purpose of this study was to test if the exposure to a stressful situation increases the activity in the teleost dorsomedial pallium (Dm) proposed as homologous to the pallial amygdala of land vertebrates. In this experiment, we stressed goldfish (Carassius auratus) by means of immobilizing them in a restraint chamber. The animals in the Restraint group $(N=8)$ were taken from the home aquaria and immobilized for 90 min between two curved plastic pads in the chamber. At the end of the restraint period, the animals were deeply anaesthetized inside the chamber. The Control animals $(N=8)$ were taken from the home aquaria and immediately deeply anaesthetized. c-Fos expression was visualized using immunohistochemistry. Representative sections used to count c-Fos-positive (c-Fos + ) cells along the rostro-caudal extent of the telencephalon were chosen at $200 \mu \mathrm{m}$ intervals. The rostral extreme of the anterior commissure was taken as the zero transversal reference point for all the brains. c-Fos + neurons were observed in the pallium, within Dm and dorsolateral pallial regions, dorsal and ventral parts (Dld and Dlv). The densities and distribution of the positive nuclei were different for Control and Restraint-stress groups. The Restraint stress situation induced a significant and important increase in c-Fos + cells density in the Dm area along its complete rostro-caudal extent $(P<0.01$ for all the analyzed levels) but not in the dorsolateral pallium (Dld and Dlv). Furthermore, the level of activation changed along the rostro-caudal axis of Dm; the highest number of c-Fos + cells was found in a region located between the 1.4 to $0.8 \mathrm{~mm}$ with respect to the zero reference, which suggests that Dm could be a heterogeneous region. We also observed a high level of c-Fos expression in the dorsal part of ventral telencephalic area, subpallial area $(\mathrm{Vd})$. We therefore quantified the number of c-Fos expressing cells in this area, showing a significant increase in the number of c-Fos positive nuclei in the Restraint animals relative to Controls along the rostrocaudal extent of this nucleus (Ps $<0.001)$. The above c-Fos expression experiments suggest that the Dm is activated during exposure to an anxiogenic situation adding functional support to its proposed homology with the amygdala.

c-Fos protein, immunohistochemistry, goldfish, pallium, restraintstress

P2-20

PARKINSONIAN PATIENTS SUFFER FROM A SERIOUS DISTURBANCE OF REDOX STATE IN THE CENTRAL NERVOUS SYSTEM, AS EVALUATED THROUGH THE CEREBROSPINAL FLUID

Angel Martin De Pablos (1), Jose Manuel Garcia-Moreno ${ }^{(2)}$, Emilio Fernandez-Espejo ${ }^{(3)}$

(1) Universidad de Sevilla, Departamento de Cirugía, Sevilla, Spain; ${ }^{(2)}$ Hospital Macarena de Sevilla-SAS, Sevilla, Spain; ${ }^{(3)}$ Universidad de Sevilla, Facultad de Medicina, Sevilla, Spain

Alteration in neurotrophic factors support and anti-oxidative defenses in the central nervous system have been associated with idiopathic Parkinson's Disease (PD). The objectives were to analyze in the cerebrospinal fluid (CSF) of patients with PD and controls: 1) the levels of the neuroprotective factors GDNF, persephin, neurturin, and BDNF, 2) the levels of TGF $\beta_{1}$ and $\mathrm{TGF} \beta_{2}$, proinflammatory factors, 3 ) the activity of the antioxidative enzymes glutathione-peroxidase, glutathione-reductase, glutathione-S-transferase, catalase, superoxide-dismutases, and peroxiredoxins, and 4) ferritin levels, iron storage protein. The study revealed that, among neurotrophic factors, only TGF $\beta_{1}$ levels were found to be enhanced in patients with PD (early, $p<0.05$; advanced, $p<0.02$ ). Regarding anti-oxidative enzymes, the activity of glutathione-peroxidase, catalase and peroxiredoxins, all hydrogen peroxide scavengers, was found to be significantly reduced in patients (glutathione-peroxidase, $p<0.001$; catalase, $p<0.01$; peroxiredoxins, $p<0.01$, one-way ANOVA). Finally, CSF ferritin content was significantly diminished over time in patients (early, $p<0.01,-49 \%$; advanced, $p<0.001,-80.7 \%$ ). Our observations lead to the hypothesis that parkinsonian patients suffer from a serious disturbance of redox state in the central nervous system, as evaluated through the cerebrospinal fluid, characterized by reduced hydrogen peroxide scavenging and iron storage.

Supported to EFE by Junta de Andalucia (BIO127) and Sociedad Andaluza de Neurología (SUBAIA2015/006; SAN).

Parkinson, Redox, CSF, hydrogen peroxide

POTENTIAL NON-CLASSICAL BIOMARKERS IN THE CEREBROSPINAL FLUID FOR THE DIAGNOSIS OF ALZHEIMER'S DISEASE

Fátima Mesa-Herrera ${ }^{(1)}$, David Quinto-Alemany ${ }^{(1)}$, Carla Ferri (1), Ana Canerina-Amaro ${ }^{(2)}$, Raquel Marin ${ }^{(2)}$, Luis GalindoMartín ${ }^{(3)}$, Mario Díaz ${ }^{(1)}$

(1) Laboratory of Membrane Physiology and Biophysics, Department of Animal Biology, Universidad de La Laguna. Associate Research Unit ULL- CSIC "Membrane Physiology and Biophysics in Neurodegenerative and Cancer Diseases", La Laguna, Spain; ${ }^{(2)}$ Laboratory of Cellular Neurobiology, Department of Basic Medical Sciences, Universidad de La Laguna. Associate Research Unit ULL- CSIC "Membrane Physiology and Biophysics in Neurodegenerative and Cancer Diseases", La Laguna, La Laguna, Spain; (3) Department of Analytical Chemistry, Universidad de La Laguna, La Laguna, Spain

Introduction: Alzheimer disease (AD) is a progressive neurodegenerative disorder which may be originated as result of genetic alterations (Familial form) or non-genetic factors (Sporadic form). Sporadic $\mathrm{AD}$ is the most common form of the disease and its causes are currently unknown. Different hypothesis indicate that a dysfunctional homeostasis of electrolytes and transition metals may play a role in the pathogenesis of $\mathrm{AD}$. It is known that aberrant homeostasis of brain copper, zinc, and particularly, iron, can generate oxidative stress, a process that has been associated to pathogenesis of sporadic AD.

Objectives: In the present work we have assessed whether variations in the composition of electrolyte and transition metals, as 
well as superoxide dismutase activity (SOD), in cerebrospinal fluid might be correlated to $\mathrm{AD}$ occurrence.

Methods: We have obtained cerebrospinal fluids from welldefined AD patients $(n=8)$ and control patients $(n=7)$. Atomic elements ( $\mathrm{Na}, \mathrm{K}, \mathrm{Ca}, \mathrm{Mg}, \mathrm{Cu}, \mathrm{Fe}, \mathrm{Mn}$ and $\mathrm{Zn}$ ) were measured by flame or graphite furnace atomic absorption spectrometry. SOD activity was measured spectrophotometrically.

Results: We have observed that $\mathrm{K}$ and Fe concentrations were significantly increased in the cerebrospinal fluid of $\mathrm{AD}$ individuals, while the concentration of $\mathrm{Zn}$ was decreased, compared to controls. Also, we observed that total proteins as well as total superoxide dismutase were increased in cerebrospinal fluids of $\mathrm{AD}$.

Conclusions: We have demonstrated disturbances in the concentrations of $\mathrm{K}, \mathrm{Fe}$ and $\mathrm{Zn}$, total protein and $\mathrm{SOD}$ activity in $\mathrm{AD}$ subjects, compared to controls. Therefore ionic markers and their relationship with classical and oxidative stress biomarkers could provide new diagnostic tools for $\mathrm{AD}$.

Alzheimer disease, biomarkers, electrolytes, superoxide dismutase, trace elements

P2-22

IMBALANCE GLUTAMATERGIC COMPOUNDS IN ANTERIOR CINGULATE CORTEX IN ADULTS AUTISM SPECTRUM DISORDER - DEMONSTRATED BY ${ }^{1} \mathrm{H}$ MRS

Carmen D. Jimenez De Espinoza ${ }^{(1)}$, Francisco Marcano Serrano ${ }^{(2)}$, José Luis González Mora ${ }^{(3)}$

(1) Universidad de La Laguna, Santa Cruz de Tenerife, Spain; ${ }^{(2)}$ IMETISA/Hospital Univ.de Canarias, Santa Cruz de Tenerife, Spain; ${ }^{(3)}$ Universidad de La Laguna/IMETISA/ Hospital Universitario de Canarias, Santa Cruz de Tenerife, Spain

Background: The L-glutamate (Glu) and L-acetyl-aspartate (NAA) are products of $\mathrm{N}$-acetyl-aspartyl-glutamate (NAAG) which require the participation of neurons, oligodendrocytes and atrocytes. On the one hand, NAAG is synthesized from NAA and Glu by an NAAG synthase, forming a dedicated pool of Glu that also cannot be further metabolized, and on the other hand, NAAG is then hydrolyzed by NAAG peptidase releasing Glu which activates the GRM3 receptor. Altered NAAG metabolism has been described, in some neurological conditions but not in autism spectrum disorders (ASD). Our previous studies using proton-Magnetic Resonance Spectroscopy $\left({ }^{1} \mathrm{H}-\mathrm{MRS}\right)$ in bilateral anterior (ACC) and posterior cingulate cortex (PCC) have described the altered neurometabolic patterns in adults with ASD.

Aim: To compare NAAG, NAA, and Glu concentrations in the ACC and PCC in adults with ASD and typically developed (control) individuals.

Methods: Single-voxel ( $\left.{ }^{1} \mathrm{H}-\mathrm{MRS}\right)$ in bilateral ACC and PCC, in 19 adults with a clinical diagnosis of ASD and 41 controls, matched for age, gender. Autism quotients (AQ) score were assessed. One-way ANOVA and Bonferroni correction were applied.
Results: The ASD group had a significant increase in glutamate $[12.10 \pm 3.92(\mathrm{mM})] * p=0.02$; as well as a significant decrease in $\mathrm{N}$-acetylaspartylglutamate $[9.78 \pm 0.49(\mathrm{mM})] * p=0.02$; compared with controls.

Conclusions: Altered $\mathrm{N}$-acetylaspartylglutamate levels were found by MRS in individuals with ASD, suggesting new therapeutic avenues.

Autism spectrum disorders, Human Brain, imaging, spectroscopy

NEUROMETABOLIC DIFFERENCE BETWEEN ANTERIOR AND POSTERIOR CINGULATED CORTICES IN AUTISM SPECTRUM DISORDERS: TARGET ON ETIOLOGY?

Carmen D. Jimenez - Espinoza ${ }^{(1)}$, Francisco Marcano ${ }^{(2)}$, José Luis González-Mora ${ }^{(3)}$

(1) Universidad de La Laguna, Santa Cruz de Tenerife, Spain; (2) IMETISA/Hospit Univ. de Canariasal, Santa Cruz de Tenerife, Spain; ${ }^{(3)}$ Universidad de La Laguna/IMETISA/Hospital Univ. de Canarias, Santa Cruz de Tenerife, Spain

Background: Phenotypic plasticity is the ability of the human brain to change its morphological patterns, interactions in its sensory modalities and patterns of release of neurotransmitters. Metabolic abnormalities of the posterior cingulated cortex (PCC) are seen in autistic spectrum disorders. In general, studies have also demonstrated reductions in functional connectivity. These reductions are particularly prominent in the $\mathrm{PCC}$, compatible with an abnormality in local circuit inhibitory processing (Oblak et al., 2011).

Aim: To study the metabolic changes in the anterior (ACC) and posterior cingulated cortices (PCC) in adults with ASD compared with typically developed individuals.

Methods: In this case-control study, in vivo single-voxel proton magnetic resonance spectroscopy signals $\left({ }^{1} \mathrm{H}-\mathrm{MRS}\right)$ were recorded in 12 right-handed young adults with ASD (median age, 22 years \pm 2.2 ), and 19 typically developing (TD) controls (mean age, $22.80 \pm 3.25$ ) who were well matched for age, and different AQ score. The Autism Spectrum Quotient (AQ) designed by Baron-Cohen et al., 2001 to assess Autistic Spectrum traits in intellectually competent adults in both the general population and the Autism Spectrum community. Imaging was performed on a 3.0-T scanner using a spectroscopy technique. The volume of interest (VOI) was located in the posterior cingulate cortex (as an important part of the Default Mode Network-DMN) and the anterior cingulate cortex. The absolute concentrations of creatine + phosphocreatine ( $\mathrm{Cr}+\mathrm{PCr}$ ), N-acetyl-aspartate (NAA), N-acetylaspartate+N-acetyl-aspartyl-glutamate (NAA+NAAG), N-acetylaspartyl-glutamate (NAAG), Glycerol-phosphocholine + phosphatidylcholine $(\mathrm{GCP}+\mathrm{PCh})$, myo-inositol $(\mathrm{mI})$, glutamate $(\mathrm{Glu})$ and glutamate + glutamine $(\mathrm{Glu}+\mathrm{Gln})$ were processed by the LC Model 6.2-3A.

Results: After the previous comparisons we decided to perform comparisons between anterior and posterior cingulated cortices on each group. In these analyses we found metabolic differences between the two regions (ACC and PCC) specifically in the ASD group. Although each region has different functions the 
control group reveals that metabolically maintain a balance, and that in the ASD group is not. Our findings shown significant lower $\mathrm{NAA} / \mathrm{Cr}(p=0.002)$ and NAA/Cho $(p=0.001)$ ratios in ACC compared with PCC in ASD group. While TD group shown no significant neurometabolic difference between ACC vs. PCC. One-way ANOVA and Bonferroni Multiple Comparison Test. $P<0.05$ were applied to perform the statistical analysis.

Conclusions: The ASD group has a highly metabolic PCC. The elucidation of the etiology of this difference is a research goal for our team.

Posterior and anterior cingulated cortices; phenotypic plasticity; proton magnetic resonance spectroscopy

$\mathrm{P} 2-24$

\section{LIPID PEROXIDATION INDUCED BY CUMENE HYDRO- PEROXIDE PRODUCES SYNAPTIC DEPRESSION IN RAT PRIMARY MOTOR CORTEX NEURONS}

Livia Carrascal ${ }^{(1)}$, Ricardo Pardillo-Díaz ${ }^{(1)}, \mathrm{M}^{\mathrm{a}}$ Eugenia MartínPachón ${ }^{(1)}$, German Barrionuevo ${ }^{(2)}$, Pedro Núñez-Abades ${ }^{(1)}$

(1) Department of Physiology. University of Seville, Sevilla, Spain; (2) Department of Neuroscience. University of Pittsburgh., Pittsburgh, United States

Pyramidal neurons of the motor cortex layer $\mathrm{V}$ that regulate voluntary control of the motor output are selectively degenerated in Amyotrophic Lateral Sclerosis (ALS). A minority of ALS cases have genetic origin due to multiple missense mutations in the cytosolic $\mathrm{Cu} / \mathrm{Zn}$ superoxide dismutase 1 gene. ALS is, however, a predominantly sporadic disorder probably triggered by multiple mechanisms. Protein misfolding and aggregation, excitotoxicity, mitochondrial dysfunction, impaired axonal transport and neurofilament aggregation, oxidative stress and lipid peroxidation has been proposed as cellular mechanism linked to motor neuron degeneration in this pathology. To understand how lipid peroxidation induces neuronal damage, whole-cell patch-clamp recordings in voltage clamp mode were obtained from pyramidal neurons of the motor cortex layer $\mathrm{V}$ in rat brain slices and changes in synaptic transmission were studied. Lipid peroxidation was induced by the administration of Cumene Hydroperoxide (10 mM HC), a stable organic oxidizing agent that is known to penetrate into the inner hydrophobic part of the membrane lipid bilayer, causing extensive peroxidation of the lipid. Spontaneous (sEPSC) and miniatures excitatory postsynaptic currents (mEPSC) were analyzed in control condition, and after $\mathrm{CH}$ application to the bath. Frequency, amplitude and EPSC shape parameters were measured. $\mathrm{CH}$ induced a decrease in sEPSC frequency that persists even after washout. However, there was no significant difference in event amplitude caused by $\mathrm{CH}$. To determine the relative contribution of presynaptic mechanisms underlying decreased sEPSC frequency and the kinetics of neurotransmitter release, TTX was added to observe action potential-independent release only. We found a decrement in mEPSC frequency indicating that the decrease in EPSC is AP-independent. A decrease in mEPSC frequency indicates a decreased release indicative of a presynaptic mechanism. No change in amplitude or time-to-peak was found in mEPSC but decay times were shortened during $\mathrm{CH}$ application. This latter finding correlates with the decrease in membrane time constant found in current clamp experiments after $\mathrm{CH}$ application, which could be due to postsynaptic effects of the $\mathrm{CH}$. Altogether, our patch-clamp data suggest that the functional changes induced by $\mathrm{CH}$ upon pyramidal neurons of the motor cortex layer $\mathrm{V}$ are due to pre- and postsynaptic mechanisms. We conclude that both the decrease in membrane time constant (which in turn may shorten decay times of the EPSCs), and the decrease of the EPSC frequency produce a synaptic depression onto pyramidal neurons of the motor cortex in oxidative stress condition that is caused by lipid peroxidation.

Amyotrophic Lateral Sclerosis, Cumene hydroperoxide, Lipid peroxidation, Patch clamp, Pyramidal neurons, Synaptic transmission.

\section{P2-25}

\section{LOCAL INHIBITION OF ADAM17/TACE PROMOTES NEUROGENESIS AFTER TRAUMATIC BRAIN INJURY}

Noelia Geribaldi-Doldán, Manuel Carrasco, Maribel MurilloCarretero, Juan Pedro Muñoz-Miranda, Francisco García-Cozar, Carmen Romero-Grimaldi, Gema García-Aguirre, Juan José Ramos-Rodríguez, María Sánchez-García, Álvaro Vidal-Suárez, Valme Del Río-Garía, Carmen Castro

\section{Facultad de Medicina, Cádiz, Spain}

Neural precursors cells (NPCs) are activated in central nervous system in response to different types of injuries. Traumatic brain injuries are accompanied by a rapid neurogenic response inside neurogenic niches. However, this response does not contribute to the generation of new neurons within the damaged area since injuries contribute to the generation of a non-neurogenic environment around the lesion area. Previously, we have demonstrated the role of the sequential activation of ADAM17/TGF $\alpha / E G F R$ signaling cascade in the generation of a gliogenic environment within brain lesions. In here, we demonstrated that chronic local infusion of a general metalloprotease inhibitor, increases the number of neuroblasts around the lesion. In addition we have created a lentivirus containing an ADAM17 negative-dominant mutant $\left(\right.$ ADAM17 $\left.\Delta_{215-473}\right)$. Adult male mice were anesthetized and controlled mechanical injuries that produced discrete lesions restricted to the gray matter in the primary motor cortex were performed. Mice were divided in 3 groups, either receiving a local injection of ADAM17 $\Delta_{215-473}$, an empty lentivirus or vehicle only. The efficacy of this therapy tool was tested at 14 and 28 days after the injury and the injection of the lentiviral vector. Local injection of this virus dramatically increased the number of neuroblasts in damaged cortical regions 14 days after injury. Those neuroblasts were able to generate new mature neurons at 28 days after injury. We have identified the phenotype of these new neurons as cholinergic, GABAergic. We conclude that ADAM17 can be targeted in order to develop new therapeuthic tools for the treatment of traumatic brain injury.

This work was funded by the Spanish Ministerio de Economía y Competitividad MINNECO (grant number: BFU2015-68652-R), Consejería de Economía Innovación, Ciencia y Empleo, Junta de 
Andalucía, España (grant numbers P10-CTS6639 P11-CTS7847), and Fundación Rodríguez Pascual 2015 grants.

ADAM17, injured brain, metalloproteases, neural stem cells, neurogenesis

P2-26

INCREASED CENTRAL BLEEDING AND COMPROMISED PROLIFERATION AND NEUROGENESIS IN A MURINE MODEL OF PRETERM INFANT GERMINAL MATRIXINTRAVENTRICULAR HEMORRHAGE

Antonio Segado Arenas ${ }^{(1)}$, Daniel José Sánchez Sotano ${ }^{(2)}$, Juan José Ramon Rodriguez ${ }^{(2)}$, Carmen Infante García (2), Simón Lubián ${ }^{(1)}$, Monica García Alloza ${ }^{(2)}$

(1) Hospital Universitario Puerta del Mar, Cádiz, Spain;

(2) Universidad de Cádiz, Cádiz, Spain

Prematurity is a worldwide health problem, with an increasing incidence of up to $10 \%$ around the world. In recent years we have improved the survival of premature infants, although the incidence of related diseases has also raised, including bronchopulmonary dysplasia or central nervous system related disorders. Among these, one of the most feared central complications is the germinal matrix-intraventricular hemorrhage (GM-IVH), affecting up to $30 \%$ of babies with extremely low birth weight $(<1000$ grs) and $15 \%$ of those with very low birth weight $(<1500 \mathrm{grs})$. On the other hand, previous studies have shown that central proliferation and neurogenesis may respond to different insults, although it remains unclear whether these mechanisms might be altered after the lesion of a neurogenic niche, as in GM-IVH.

In order to further characterize GM-IVH and its long-term effects on central proliferation and neurogenesis, we have induced mild or severe GM-IVH by injecting 0.1 or 0.3 IU of proteolytic enzyme collagenase, into the right ventricle of $\mathrm{p} 7$ (equivalent to 28 weeks of human gestation) CD1 mice. Sham animals followed the same protocol and they received PBS instead. Animals were allowed to complete development into adulthood and received ip BrdU $(70 \mathrm{mg} / \mathrm{kg}$ ) for 3 consecutive days immediately before sacrifice at $\mathrm{p} 70$. We quantified the presence of hemorrhages by Prussian blue staining and we also analyzed central proliferation and neurogenesis by BrdU and doublecourtin immunostaining. Hemorrhage burden was increased in a dose-dependent manner after collagenase lesions in $\mathrm{p} 70$ mice. Cell proliferation was maintained in the subventricular zone and the dental gyrus, while neurogenesis was significantly reduced after collagenase lesions in 770 mice. When we quantified the cortex and whole hippocampus we detected an overall dose-dependent reduction in the number of BrdU and doublecourtin positive cells after collagenase lesions.

Altogether our data show that intracerebroventricular collagenase administration provides a useful tool to recreate a GM-IVH in a murine model, showing bleeding and reduced neurogenesis. Moreover, these outcomes are chronically observed, suggesting a long-term effect of this type of perinatal lesions.
Acknowledgements: MG-A: Fundación Eugenio Rodriguez Pascual 2015. Junta de Andalucia, Proyectos de Excelencia (P11-CTS-7847); Instituto de Salud Carlos III and FEDER (European Union), co-financed by Fondo Europeo de Desarrollo Regional "Una manera de hacer Europa" (PI12/00675).

collagenase, preterm neonate, germinal matrix hemorrhage, proliferation, neurogenesis, subventricular zone

P2-27

INSULIN RESCUES LONG-TERM CENTRAL COMPLICATIONS IN A MURINE MODEL OF OFFSPRING FROM TYPE I DIABETES MOTHERS

Juan Jose Ramos Rodríguez, Alberto Doblas Márquez, Carmen Infante García, Daniel José Sánchez Sotano, Mónica García Alloza

Universidad de Cádiz, Cádiz, Spain

Diabetes mellitus (DM) is one of the most common chronic diseases nowadays, with a prevalence of 387 million diabetics worldwide. Type I diabetes (T1D) is the second most common cause of diabetes and it is characterized by the selective destruction of pancreatic $\beta$ cells, in most cases due to an autoimmune mechanism, causing an insulin deficit. Previous studies have shown that T1D may lead to central complications such as cognitive problems, cortical atrophy or stroke. However, the potential long-term effects of T1D, in pregnant women, on their offspring central nervous system are not entirely clarified. In order to further explore the relationship between T1D during the pregnancy and central pathology in their offspring, we have induced T1D in CD1 mice by streptozotozin (STZ) administration immediately before pregnancy.

Puppies from T1D mothers showed severe brain atrophy, that affected the cortex and hippocampus, and this effect was reversed by intracerebroventricular or intranasal insulin administration. Similarly, reduced neuronal density in offspring from T1D mothers was rescued by insulin administration. Insulin also recovered neuronal density in treated offspring from diabetic mothers. Moreover, at subcellular level, neuronal complexity was significantly affected in puppies from STZ-treated mothers and insulin reversed this situation. While local intracerebroventricular administration rescued observed central pathological features, intranasal administration resulted in a complete reversion of all deficits under study, supporting the role of this administration pathway to successfully access the central nervous system.

Altogether, our data suggest that intracerebroventricular or intranasal insulin treatment could improve the prognosis of infants from T1D mothers in the long term.

Acknowledgements: MG-A: Fundación Eugenio Rodriguez Pascual 2015. Junta de Andalucia, Proyectos de Excelencia (P11-CTS-7847); Instituto de Salud Carlos III and FEDER (European Union), co-financed by Fondo Europeo de Desarrollo Regional "Una manera de hacer Europa" (PI12/00675).

Type 1 diabetes, offspring, insulin, intracerebroventricular administration, intranasal administration, neuronal simplification 
P2-28

A HIGH-FAT DIET IN RATS PRODUCES CHRONODISRUPTION IN PHAGOCYTIC ACTIVITY IN NEUTROPHILS

Rafael Bravo (1), Lierni Ugartemendia ${ }^{(1)}$, Lourdes Franco ${ }^{(1)}$, Mónica Mesa ${ }^{(1)}$, Ana B. Rodríguez ${ }^{(1)}$, Javier Cubero ${ }^{(2)}$, Carmen Barriga $^{(1)}$

(1) Department of Physiology. Faculty of Science. University of Extremadura., Badajoz, Spain; ${ }^{(2)}$ Experimental Science Education Area. University of Extremadura., Badajoz, Spain

Introduction: Obesity is an epidemic disease in all around the world with many deleterious effects on life quality. It has been reported that obesity affects to immune system. These alterations during obesity have been appointed to cytokines imbalance which provokes a chronic inflammation which effect on leucocytes. Objectives: Our aim was to elucidate if a high-fat diet may produce chronodisruption in phagocytosis circadian rhythm in neutrophils. Materials \& Method: 42 wistar rats were fed another high-fat diet (60\% lipids) for 11 weeks, another control group $(n=42)$ was considered for the same period. At the end of the 11th week rats were sacrified at 00:00, 02:00, 04:00, 10:00, 14:00, 18:0 and 22:00 h (6 rats per group). After sample collections we measured phagocytic activity in neutrophils and circadian rhythm variables were calculated with the software Ritme ${ }^{\mathbb{R}}$

Results: Our results showed that control group had a circadian rhythm in phagocytic activity in plasma neutrophils (MESOR: 18.17, Amplitude: 7.43 , Acrophase: $3: 14 \mathrm{~h} ; p<0.01$ ). On the other hand, high-fat fed rats did not show a circadian rhythm in neutrophils phagocytosis and even showed higher values (MESOR: 27.34, Amplitude: 3.42 , Acrophase: 6:05 h; $p=0.48$ ).

Conclusion: Our data revealed that high-fat diets may lead to a chronodisruption in immune functions such as phagocytic activity. Acknowledgments: Authors want to express their gratitude to Junta de Extremadura (Fondos FEDER: BB021- GR15051).

Chronobiology, chronodisruption, immune system, high-fat diets, rats,

P2-29

IMPACT OF BISPHENOL-A TREATMENT DURING PREGNANCY ON MICE KIDNEY: A PRELIMINARY STUDY

Paula Núñez ${ }^{(1)}$, María Teresa Fernández ${ }^{(1)}$, Marta García-Arévalo (2), Paloma Alonso-Magdalena ${ }^{(2)}$, Ángel Nadal ${ }^{(2)}$, Carmen Perillán ${ }^{(1)}$, Juan Argüelles ${ }^{(1)}$

${ }^{(1)}$ Universidad de Oviedo, Oviedo, Spain; ${ }^{(2)}$ Universidad Miguel Hernández, Elche, Spain

The developmental exposure to endocrine-disrupting chemicals such as bisphenol A (BPA) contributes to an increase in various metabolic diseases incidence, including type 2 diabetes. In the literature, animal studies report a nephron deficit in offspring exposed to maternal diabetes. The nephron is the basic structural and functional unit of the kidney, so this reduced nephron number may produce glomerular hyperfiltration that is associated with augmented glomerular hydrostatic pressure and renal hypertrophy, increasing the risk for adult development of renal and cardiovascular diseases.

The aim of this preliminary study was to investigate prenatal BPA exposure effects on renal function in an animal model. This study quantitatively evaluated the renal structural changes by means of stereology and histomorphometry methods.

OF1 pregnant mice were treated with either vehicle or BPA (10 or $100 \mathrm{mg} / \mathrm{kg} /$ day) during days 9 to 16 of gestation. The 30 -day-old offspring were sacrificed and tissue samples were collected. Kidneys were dissected and prepared for light microscopy, histomorphometry and stereology studies. The ethical committee of Miguel Hernandez University "Comisión de Ética en la Investigación Experimental" specifically reviewed and approved this study (UMH-IB-AN-01-14).

Glomerular abnormalities and reduced glomerular formation have been observed in BPA-treated offspring. The kidneys of the BPA10 and BPA100 treated-offspring had significantly fewer glomerular number and density than CONTROL offspring. Glomerular histomorphometry revealed significant difference between females and males of the CONTROL offspring with respect to the analyzed glomerular parameters (mean glomerular diameter, glomerular volume). This difference disappeared in the BPA10 and BPA100 treated-offspring. We also observed in treatment groups a glomerular enlargement and tubular dilation without evidence of other damages. Additionally, kidney histopathological examination showed the typical male cuboidal epithelial cells of the Bowman's capsule in the female BPA-treated offspring.

All these data suggest that the exposure to environmentally relevant doses of BPA during embryonic development may alter nephrogenesis. Further studies are needed to clarify the potential role of BPA in the pathogenesis in the progression of renal diseases, particularly in diabetic patients.

Funding: MINECO BFU2011-28358

Kidney,bisphenol-A,mice,pregnancy

P2-30

\section{CARDIOTROPHIN-1 UPREGULATES EXTRACELLULAR MATRIX PROTEIN EXPRESSION}

Nuria Perretta Tejedor ${ }^{(1)}$, Jose Manuel Munoz Felix ${ }^{(2)}$, Cristina Cuesta Apausa ${ }^{(1)}$, Isabel Fuentes Calvo ${ }^{(2)}$, Nelida Eleno ${ }^{(2)}$, Jose Miguel Lopez Novoa ${ }^{(2)}$, Carlos Martinez Salgado ${ }^{(1)}$

(1) TRECARD, Departamento de Fisiologia, USAL, IESCYL,IBSAL, Salamanca, Spain; ${ }^{(2)}$ TRECARD, Departamento de Fisiologia, USAL, IBSAL, Salamanca, Spain

Renal tubule-interstitial fibrosis is characterized by myofibroblast proliferation and activation in the renal interstitium, and extracellular matrix (ECM) synthesis. Transforming growth factor-b1 (TGF- $\beta 1$ ) is the main profibrotic cytokine involved in this process. Cardiotrophin-1 (CT-1), a member of the interleukin-6 (IL-6) family of cytokines, has a protective effect in several organs, promoting survival or antinflamatory effects. We have previously shown that $\mathrm{CT}-1^{-/}$mice showed higher renal fibrosis than WT mice after 15 days of unilateral ureteral obstruction (UUO), an 
experimental model of tubule-interstitial fibrosis. In addition, CT-1 treatment reduced renal fibrosis in WT mice. Thus, we analysed the effect of CT-1 treatment at the severity of renal tubuleinterstitial damage induced by UUO, in animals lacking CT-1 (KO). We also analysed the CT- 1 and TGF- $\beta 1$-induced effects on ECM protein synthesis in renal myofibroblasts obtained from obstructed kidneys in WT and KO mice

UUO was performed in CT- $1^{-/-}$mice and their respective controls (WT) during 15 days. Cultured myofibroblasts were stimulated with $40 \mathrm{ng} / \mathrm{ml} \mathrm{CT}-1$ and with $1 \mathrm{ng} / \mathrm{ml}$ TGF- $\beta 1$ during $24 \mathrm{~h}$.

We observed a higher CT-1 expression in obstructed (O) than in non-obstructed (NO) kidneys after 15 days of UUO in WT mice. CT-1 treatment (100 and 400ug/kg) in CT-1 KO mice reduced collagen I, CTGF, fibronectin, $\alpha \mathrm{SMA}$ and PCNA expression as well as Sirius red staining in O kidneys after 15 days UUO. On the other hand, the in vitro studies showed that renal myofibroblasts express CT-1. In basal conditions, CT-1 KO cells show a higher expression of collagen I and fibronectin than WT cells. Stimulation with TGF- $\beta 1$ increases CT- 1 expression in WT cells and leads to a higher expression in collagen I and fibronectin in KO than in WT cells. Stimulation with CT-1 increases expression of collagen I, however, co-stimulation with CT- 1 and TGF- $\beta 1$ blocks TGF- $\beta 1$ induction of collagen I.

This study shows that CT- 1 regulates renal fibrosis induced by obstructive nephropathy in $\mathrm{KO}$ mice and it also shows that endogenous CT-1 regulates the expression of ECM proteins in vitro. We suggest that the protective effects of CT-1 administration are related with a blockade of the TGF- $\beta 1$ intracellular pathway.

Cardiotrophin-1, renal fibrosis, extracellular matrix

P2-31

CLONING AND CHARACTERIZATION OF PHOSPHATE TRANSPORTERS IN OK-P CELLS

Susana Lucea Arnas, Natalia Guillen Monzon, Victor Sorribas Alejaldre

Universidad de Zaragoza, Zaragoza, Spain

Pi transport through epithelia has been extensively studied, and several $\mathrm{Na}+-$ dependent $\mathrm{Pi}$ transporters have been identified. These transporters are very frequently expressed in the same cell, and the need for such apparent redundant function remains unclear. In this work we have identified, characterized and studied the regulation of three sodium-dependent Pi transporters involved in Pi uptake that were still not sequenced, in a classical model of renal proximal tubule: the Opossum kidney (OK-P) cell line, from Didelphis virginiana.

The type II Na-Pi transporter NaPi-2c, and the two type III transporters (Pit-1 and Pit-2) were amplified using RNA from OK cells and RNA ligase-mediated (RLM-RACE) oligo-capping rapid amplification of cDNA ends (RACE) techniques. The cDNAs were cloned, sequenced, and analyzed for homology with other species. $32 \mathrm{P}$ uptake and the specific mRNA and protein content of the transporters were studied in OK-P cells under different conditions. To study the regulation by phosphate deprivation, the cells were incubated for $24 \mathrm{~h}$ in DMEM with $0.1 \mathrm{mM}, 1 \mathrm{mM}$ and $2 \mathrm{mM}$ Pi.
$\mathrm{Na}+-$ dependent 32Pi uptake was then determined, which was, as expected, significantly increased in the cells incubated with low $(0.1 \mathrm{mM})$ Pi concentration in DMEM.

The involvement of the different Na-Pi transporters in Pi uptake in OK-P cells was then studied. Analysis of the previously identified $\mathrm{NaPi} 2 \mathrm{a}(\mathrm{NaPi} 4$ in $\mathrm{OK})$ protein expression showed that the protein abundance changes paralleled those of Pi transport. We failed to produce valid antibodies against the other three transporters. With respect to RNA, the type II Na-Pi transporters showed higher expressions than type III transporters under basal conditions (1 mM Pi), which was confirmed by transport studies: OK-P showed higher $\mathrm{Pi}$ uptake at alkaline than acidic $\mathrm{pH}$, suggesting the preference of HPO42- (a type II substrate) over H2PO4- (a type III substrate).

The use of the transcription inhibitor actinomycin D (AD) for $24 \mathrm{~h}$ clearly inhibited Pit1, Pit2 and NaPi2c mRNA abundance, and had no effect on NaPi4 mRNA expression but it prevented transport adaptation to Pi depletion. Total Pi uptake at pHs 6.0 and 8.5 was also significantly inhibited by $\mathrm{AD}$ incubation as well. $5 \mathrm{mM}$ phosphonoformic acid (PFA), a specific inhibitor of type II Na$\mathrm{Pi}$ transporters uptake, partially decreased $\mathrm{Pi}$ uptake at $\mathrm{pH} 8.5$ but not at $\mathrm{pH}$ 6. These findings corroborate the role of type II Na-Pi transporters as the main Pi transporters in the proximal tubule, but the specific relevance of the transporters are being assayed with siRNAs.

Opossum Kidney cells, Pi transport, RACE cloning, NaPi2c, PiT1, PiT2

P2-32

ASSOCIATION OF PLASMA CYTOKINE LEVELS WITH CLINICAL RENAL PARAMETERS IN PATIENTS WITH METABOLIC SYNDROME

Isabel Sánchez-Vera, Cira García De Durango, Roberto OrtízRegalón, José Felipe Varona

Universidad San Pablo CEU, Boadilla del Monte, Madrid, Spain

Introduction: Chronic kidney disease (CKD) is associated with increased risk for cardiovascular morbidity and mortality. The development of CKD is commonly the result from the interactions among hemodynamic factors and a cluster of risk factors, as insulin resistance, visceral obesity, hypertension and dyslipidemia, all of them components of the metabolic syndrome (MetS). In the present study we analyze in patients with risk factors for MetS renal clinical data and their correlation with plasma cytokine levels and MetS components, in order to determine possible links between MetS and development of CKD.

Methods: 71 patients with components of MetS according to NCEP ATP III criteria were included. None of them had established kidney disease. Plasma concentrations of cytokines and the soluble cell adhesion molecules were determined on the Luminex-100 system (Luminex Corporation). Clinical renal data were analyzed: urine albumin to creatinine ratio (UACR), plasma uric acid (UA), plasma creatinine and glomerular filtration rate (GFR) estimated by MDRD formula. 
Results: Plasma creatinine correlated with leptin $(r=-0.305$; $p=0.017)$ and PAI-1 $(r=0.262 ; p=0.039)$; UA correlated with ICAM-1 $(r=0.328 ; p=0.007)$, resistin $(r=0.235 ; p=0.05)$, PAI-1 $(r=0.411 ; p=0.001)$ and MCP-1 $(r=0.246 ; p=0.046)$. Patients with abdominal obesity $(n=49)$ had lower plasma creatinine level $(0.88 \pm 0.18 v s 1.14 \pm 0.3 ; p=0.001)$ and higher GFR (94.88 \pm 17.02 vs $78.95 \pm 20.57 ; p=0.007)$ than patients without it $(n=12)$. In contrast, patients with hypertriglyceridemia $(n=42)$ showed higher plasma creatinine level $(0.99 \pm 0.25$ vs $0.84 \pm 0.16$; $p=0.01)$, lower GFR $(86.86 \pm 19.71$ vs $98.55 \pm 14.62 ; p=0.013)$ and higher UA $(6.87 \pm 1.14$ vs $5.7 \pm 0.99 ; p=0.001)$ than patients without it $(n=26)$. There was no statically difference in renal parameters between patients with or without hypertension, impairment fasting glucose or low cholesterol HDL.

Conclusions: Leptin, PAI-1, ICAM-1, resistin and MCP-1 were the cytokines related to renal clinical data, supporting their pathophysiological role in the proinflammatory and prothrombotic status of the patients with high risk of developed a CKD. With respect to MetS components, hypertriglyceridemia was the only one clearly associated with renal dysfunction.

Chronic kidney disease, Metabolic Syndrome, cytokines, renal dysfunction.

P2-33

ROLE OF CARDIOTROPHIN-1 IN THE DEVELOPMENT OF RENAL COMPENSATORY HYPERTROPHY AFTER UNILATERAL NEPHRECTOMY IN MICE

Cristina Cuesta Apausa ${ }^{(1)}$, Nuria Perretta-Tejedor ${ }^{(1)}$, María Paniagua-Sancho ${ }^{(2)}$, José M López-Novoa ${ }^{(2)}$, Nélida Eleno ${ }^{(2)}$, Francisco J López-Hernández ${ }^{(1)}$, Carlos Martínez-Salgado (1), Isabel Fuentes-Calvo ${ }^{(2)}$

(1) Instituto de investigación Biomédica de Salamanca, S, Spain;

(2) Universidad de Salamanca, Salamanca, Spain

Introduction: Compensatory renal hypertrophy occurs in renal disease as a consequence of the reduction in the number of functioning nephrons. This process is more important following partial or complete uninephrectomy (UNX) and very relevant after renal transplantation in both living donors and recipients. An excessive compensatory hypertrophy could be determinant for progression of renal disease, so the knowledge of signals and mechanisms that regulate this process might limit the progressive kidney damage. Cardiotrophin-1 (CT-1) is a molecule widely studied for its hypertrophic effects in heart. We hypothesized that CT-1 could modulate renal hypertrophy after UNX and thus have a protective role against renal impairment.

Methods: Left nephrectomy was performed in $\mathrm{CT}^{-/}$mice and WT controls during 3 and 8 days; mice were treated with $400 \mathrm{mg} / \mathrm{Kg}$ mouse CT- 1 or $50 \mathrm{mg} / \mathrm{Kg}$ anti-mouse CT- 1 antibody for $24 \mathrm{~h}, 4$ and 6 days post- nephrectomy to evaluate the short-term role of CT-1. Moreover, left nephrectomy was performed in $\mathrm{CT}^{-/-}$mice and WT controls for 7 months to evaluate the long-term role of CT-1. At the end of each period, the ratio kidney weight/body weight (BW) was calculated. Also PCNA expression, to confirm the development of hypertrophy instead hyperplasia, was analyzed.
Fibronectin, collagen and $\alpha$-SMA expressions were analyzed to evaluate the presence or not of fibrosis.

Results: Uninephrectomy increased the ratios kidney/BW in both WT and $\mathrm{CT} 1^{-1}$ mice after 3,8 days and 7 months, without any significant difference between groups. After 8 days nephrectomy, treatment with CT-1 or anti- CT1 antibody did not have any effect on kidney mass either in sham or in uninephrectomiced mice. No difference were observed in PCNA, fibronectin, $\alpha$-SMA and collagen expressions between uninephectomiced WT and $\mathrm{CT}^{-1-}$ mice. Therefore cardiotrophin-1 does not participate in the development of compensatory renal hypertrophy. Moreover the absence of cardiotrophin does not induce fibrosis after unilateral nephrectomy in rats.

Conclusions: Our results showed that the compensatory renal hypertrophy induced after unilateral nephrectomy is not regulated by CT-1.

Cardiotrophin-1, compensatory renal hypertrophy, fibrosis

P2-34

GPR30 EFFECTS ON RENOVASCULAR FUNCTION

Alvaro Casanova, Yaiza Marzo, Desiree Pereboom, Jose Octavio Alda

Universidad de Zaragoza, Zaragoza, Spain

The recent discovery of the new receiver GPR 30 , a G proteincoupled receptor for estrogens, has led to create the research field for non-genomic effects of steroid hormones. However, we still are not able to conclude its effects on the kidney. GPR30 was located predominantly in the endothelium of smooth muscle of inter-renal vessels, whereas dispersed markings have been found in the cortex. The functions mediated by GPR30 share an effective hypotension on the whole organism.

Materials and methods: The kidney is an outstanding target in drug effect studies. The isolated perfused kidney technique provides us with an ex vivo model, and it allows to test different agents which interfere with the GPR30 receiver. It is pharmacologically interesting to find different compounds with selective estrogenic activity to GPR30, which do not mediate in the classical receiver's action. There are both natural and synthetic options, so we decided to use estrogen agonists: genistein (phytoestrogen, with a natural origin), the strogens G1 $\left(\mathrm{C}_{21} \mathrm{H}_{18} \mathrm{BrNO}_{3}\right.$, highly selective with synthetic origin) and $17 \beta$-Estradiol (E2, the most potent mammalian estrogenic steroid) plus an antagonist, $\mathrm{G} 15\left(\mathrm{C}_{19} \mathrm{H}_{16} \mathrm{NBrO}_{2}\right.$, with a synthetic origin).

Results: E2 produces a visible vasorelaxation by decreasing a $24.39 \%$ the Renal Vascular Resistance (RVR) at a dose of $50 \mu \mathrm{M}$. A $50 \mu \mathrm{M}$ concentration of G1 causes a $12.27 \%$ decrease of RVR, a $183.14 \%$ increase of diuresis and a $171.89 \%$ increase of natriuresis. G15 as an antagonist of GPR 30 causes constriction itself, increasing the $\%$ of RVR in a $11.60 \%$. The addition of the G15 to kidney treated with G1, E2 and genistein decreased the relaxing effect of these estrogens on $6.47,21.63$ and $29 \%$ respectively. 
Conclusion: In our model of isolated perfused kidney, E2 and G1 treatment significantly decreased renal vascular resistance.

Furthermore, our results suggest the involvement of GPR30 in RVR regulation.

GPR30, Genistein, G1, E2, G15, Vasorelaxation

P2-35

NRK-52 RENAL CELL LINE CISPLATIN SENSITIVITY: RELATIONSHIP TO GGT1 ACTIVITY AND SHEAR STRESS

Cristina Arias ${ }^{(1)}$, Natalia Sanchez-Romero ${ }^{(2)}$, Laura MartinezGimeno ${ }^{(2)}$, Miriam Esteban (1), Alina Khramova ${ }^{(3)}$, Patricia Meade ${ }^{(3)}$, Ignacio Gimenez ${ }^{(2)}$

${ }^{(1)}$ Instituto Aragones Ciencias Salud, Zaragoza, Spain; ${ }^{(2)}$ Instituto Aragones Ciencias Salud-IISAragon, Zaragoza, Spain; (3) Universidad de Zaragoza, Zaragoza, Spain

Our long-term aim is the generation of a biomimicking cell culture system to study human proximal tubule cells (PT) damage and regeneration mechanisms. Here we describe our method to analyze, in living cells, their viability and the activity of gammaglutamyl transferase (GGT1), an enzyme involved in cell defense against oxidative stress.

NRK-52, a rat cell line of renal origin is used as a model for proximal tubule cells. NRK cells are grown in 96 well plates and exposed to a well-known nephrotoxicant (cisplatin, CDDP) $48 \mathrm{~h}$ before the assay. Cell viability is determined using resazurin reduction method (PrestoBlue). A colorimetric assay for GGT1 activity takes advantage of GGT1 transpeptidase activity.

We have optimized the assay conditions to allow us determine both reactions sequentially and to use culture medium as the assays solution, so that cells can be monitored over time by repeated assaying. NRK-52 cells exhibit cisplatin sensitivity with an LD50 of 50-60 $\mu \mathrm{M}$. Cell viability and cell number (determined by crystal violet staining) decrease as cisplatin doses increase. However, we did not observe a corresponding change in GGT1 activity. A cytochemistry staining for GGT1 activity revealed a large heterogeneity of GGT1 activity in control NRK-52 cells, suggesting the simultaneous existence of different phenotypes despite a rather homogeneous morphology. Cisplatin exposure caused an increase in the observed ratio of GGT1 positive cells. Thus, either GGT1 positive NRK cells are resistant to cisplatin or cisplatin exposure induces GGT1 activity as a defense against oxidative stress. These mechanisms are relevant to our understanding of the biology of PT cells but are also shared by many tumoral cells that become resistant to cisplatin.

To introduce the physiological parameter of fluid-flow mediated shear stress, we have adapted our method for its use with cells growing inside microfluidic channels. NRK cells tolerated well being cultured under these conditions. However, a few parameters needed to be adjusted to replicate the same NRK cells response to cisplatin observed in the 96 -well format.
Financial Support: Spain's Ministerio de Economía, grant DPI2011-28262/C02 and fellowship BEP-2012-059562 to NS-R

Nephrotoxicity, in vitro cell models, kidney, gamma-glutamyl transferase, NRK52

P2-36

HETEROGENEOUS PHENOTYPE IN LONG-TERM PRIMARY CULTURES OF HUMAN PROXIMAL TUBULE: EFFECTS OF ECM

Miriam Esteban ${ }^{(1)}$, Natalia Sanchez-Romero ${ }^{(2)}$, Laura MartinezGimeno ${ }^{(2)}$, Pilar Torcal ${ }^{(2)}$, Manuel Sanchez-Zalabardo ${ }^{(2)}$, Pablo Íñigo ${ }^{(2)}$, Ignacio Gimenez ${ }^{(2)}$

${ }^{(1)}$ Instituto Aragones Ciencias Salud, Zaragoza, Spain; ${ }^{(2)}$ Instituto Aragones Ciencias Salud-IISAragon, Zaragoza, Spain

Primary cultures are a valuable tool to study function and regulation of specialized tissues in vitro. However, one of their limitations is the difficulty to isolate a pure population. For the kidney, this is even more difficult given the number of different cell types forming every nephron. Here we report our efforts in characterizing the cell populations found in primary cultures of human renal cortex, which we term hPTPC because the proximal tubule contributes the largest fraction of initial tissue.

We have studied the expression of epithelial and mesenchymal markers, and of markers for specific nephron segments at different levels: transcripts (RT-PCR, qPCR), protein (IF, WB) and activity (gamma-glutamyl transferase 1, GGT1 assays). The effects of culture length and of ECM-like substrates composition and geometry were observed and analyzed.

At least four different cell populations are present in our hPTPC cultures. These cell types are distinguished by their morphology, expression of epithelial and mesenchymal markers, and levels of GGT1 activity. One cell type is clearly negative for GGT1 activity and shows strong expression of epithelial markers ZO-1 and CK19. Preliminary results suggest it may be originated from the distal nephron. Accordingly, RT-PCR analysis demonstrates the presence of the sodium transporter specific to the distal convoluted tubule. Other cell types appear to represent intermediate stages of epithelial-tomesenchymal transition of proximal tubule cells. We observed a positive effect on differentiation of maintaining the cultures for 14 days over 7 days. GGT1 activity in 14 days cultures was two-fold that determined in the 7 days cultures. On the other hand, there were no significant changes dependent on the kind of ECM used as coating substrate (collagen I, collagen IV, Matrigel).

Our results confirm the heterogeneity of human renal cortex primary cultures. The identity of the different cell types is more evident when cultures are maintained for longer periods of time, something that is not very commonly done. A detailed analysis revealed that a small fraction of our cells retain a pure epithelial phenotype and confirm previous observations that cells in culture undergo epithelial to mesenchymal transition as an adaptation to in vitro conditions. We are currently studying how to reduce this process by providing more biomimicking conditions through the use of 3D scaffolds and microfluidics. 
Financial Support: Spain's Ministerio de Economía, grant DPI2011-28262/C02 and fellowship BEP-2012-059562 to NS-R

Kidney, proximal tubule, primary cultures, gamma-glutamyl transferase

P2-37

\section{INDEPENDENT PHOSPHOREGULATORY DOMAINS IN NKCC2 N-TERMINUS}

Carmen Ibañez ${ }^{(1)}$, Laura Martinez-Gimeno ${ }^{(2)}$, Patricia Meade ${ }^{(1)}$, Ignacio Gimenez ${ }^{(2)}$

(1) Universidad de Zaragoza, Zaragoza, Spain; (2) Instituto Aragones Ciencias Salud, Zaragoza, Spain

Kidney specific Na-K-Cl cotransporter (NKCC2, Slc12A1 gene) is expressed at the apical membrane in cells of the thick ascending limb (TAL) of Henle's loop. NKCC2's role in water and electrolyte homeostasis is critical, and is the target for widely-used loop diuretics. . AMP-activated kinase (AMPK) acts as a physiological regulator of NKCC2, but the relationship of this pathway to other regulatory pathways is not fully understood.

In this work we analyzed in detail the regulatory role of residues in the $\mathrm{N}$-terminal cytosolic domain of $\mathrm{NKCC} 2$. NKCC2 cDNA cloned in a vector for heterologous expression in Xenopus leavis oocytes was subjected to site-directed mutagenesis to introduce single amino acid substitutions in phosphoregulatory domains. Mutation effects were assessed by quantifying rubidium uptake. Specific threonines phosphorylation was determined by Western blotting. NKCC2 exhibits maximal stimulation and threonine phosphorylation when oocytes are incubated in hypertonic solutions or coexpressed with WNK kinases. Conversely, NKCC2 activity is reduced to $50 \% \max$, and threonine phosphorylation turns negligible, when incubated in hypotonic medium.

We first confirmed that the main differences in regulation between NKCC2 and the ubiquitously expressed NKCC1 are due to divergent sequences in the $\mathrm{N}$-terminal region of the transporter, although the involvement of the transmembrane and $\mathrm{C}$-terminal regions was required for a complete activation profile.

There are two phosphoregulatory domains in the $\mathrm{N}$-amino terminal region in NKCC2, which act independently in response to different stimuli. Substitution by alanines of threonines T99, T104 and T117 abrogated the changes induced by hypertonicity and those of WNK and SPAK kinases. Ser126 replacement was shown to be required for activity under hypotonic conditions. Other potential phosphoacceptors such as Ser90 and T94 do not appear to be important for NKCC2 activity.

In the absence of S126, there were no changes in NKCC2 regulation by the WNK-SPAK pathway, suggesting this mechanism is independent of S126 phosphorylation. This result was obtained independently of whether the kinase under study was WNK3 or WNK4. In contrast to previous studies that had shown the need for SPAK co-expression, expression of WNK4 alone was able to induce changes in
NKCC2 activity and to increase the phosphorylation of threonines T99-T104.

Our results confirm an independent regulatory role for S126 phosphorylation, which has been shown to be mediated in vivo by kinases like AMPK and PKA.

Financial Support: Spain's Ministerio de Economía, grant DPI2011-28262/C02

Kidney, thick ascending limb, NKCC2, AMPK

P2-38

THE EFFECT OF A SIX-MONTH PHYSICAL ACTIVITY AND NUTRITION EDUCATION PROGRAM ON HEALTH PARAMETERS, PHYSICAL ACTIVITY AND BODY COMPOSITION IN HEALTHY YOUNG WOMEN

Roberto Ramírez, Teresa Mingo, Isabel Bayona, Mariángeles Ferrer, Enrique Márquez, Francisco Navas, Lucía Pérez-Gallardo. UVA, SORIA, Spain

It was designed an educational intervention program on nutrition and physical activity (6 months) in order to observe its effect on general and specific biochemical parameters; anthropometric parameters; body composition; fitness $\left(\mathrm{VO}_{2}\right.$ $\max$ ) and level of physical activity in college women.

Subjects and methods: 28 healthy women were included, aged 18-28. The serum glucose, HDL cholesterol, LDL cholesterol, total cholesterol, triglycerides, prealbumin, albumin, leptin and production of malondialdehyde (MDA); body mass index (BMI); body composition; $\mathrm{VO}_{2}$ max and exercise performance (hours/week) were evaluated before and after the intervention educative program.

Results: In the participants leptin increased from mean (SD) 7.2 (3.4) to $11.7(4.8) \mathrm{ng} / \mathrm{mL}(p<0.001)$. The albumin concentration increased from $4.6(0.2)$ to $4.7(0.2) \mathrm{mg} / \mathrm{dL}(p<0.05)$. Both time spent walking as time spent doing vigorous physical activity decreased from $7.36(4.03)$ to 6.33 (3.7) hours/week $(p<0.05)$ and from $1.65(2.71)$ to $1.02(1.38)$ hours/week $(p<0.05)$ respectively, and increased time spent doing moderate intensity activity from 1.37 (2.08) to $2.96(2.23)$ hours/week $(p<0.001)$. Before the intervention a negative correlation of leptin with the $\%$ water $(r=-0.664 ; p<0.001)$ and positive correlations of leptin with BMI $(r=0.582 ; p<0.01)$ and $\%$ fat mass $(r=0.659 ; p<0.001)$ respectively were observed. These correlations remained after the intervention and also negative correlations between leptin and $\mathrm{VO}_{2} \max (r=-0.498 ; p<0.05)$, between albumin and triglycerides $(r=-0.404 ; p<0.05)$ and between albumin and MDA $(r=-0.419 ; p<0.05)$ were observed.

Conclusions: Following this program contributed to change the level of physical activity of the participants and an increase in serum albumin and leptin was also noted. However, it did not significantly alter the biochemical parameters related to health, body composition or level of oxidative stress.

Biochemical health parameters; body composition; fitness; physical activity and leptin 
P2-39

ANALYSIS OF THE ELECTROMYOGRAPHIC ACTIVITY OF THE QUADRICEPS FEMORIS APPLYING KINESIOTAPING AND ELECTROTHERAPY IN TWO GROUPS OF UNIVERSITY STUDENTS: SEDENTARY AND ATHLETES

Fanny Izchel Cepeda Salazar, Emmanuel Salvador Hernández Alvarado, Cinthia Samantha Carrillo Alcantar, Miguel Angel Rivas García, Ivon Analleli De Paz Garduño, Virgilio Eduardo Trujillo Condes

Facultad de Medicina, Universidad Autónoma del Estado de México, Toluca, Estado de México, Mexico

Kinesiology taping (KT) uses elastic tape that is fixed onto the skin. Kinesio Tape is thinner and more elastic than conventional tape, which is hypothesised to allow greater mobility and skin traction. The possible mechanism of increasing proprioceptive function with KT was cutaneous afferent stimulation through the skin. Neuromuscular electrical stimulation (NMES) is the application of electrical current to elicit a muscle contraction and seems to be helpful for strengthening the muscles. The objective of this study was to asses, the effect of KT and NMES on surface electromyographic (sEMG) activity of the Quadriceps Femoris Muscle in two groups of healthy universitary females: basketball players and sedentary students. Ten basketball players (age: 19.8 \pm 1.22 years; BMI $24.6 \pm 3.43 \mathrm{~kg} / \mathrm{m}^{2}$ ) and ten sedentary students (age: $20.1 \pm 1.28$ years; BMI $22.8 \pm 3.52 \mathrm{~kg} / \mathrm{m}^{2}$ ) were enrolled in the research and submitted to the qualification procedures. The individuals in both groups received neuromuscular stimulation procedures in right quadriceps (four electrodes on the muscle attachment sites). A portable electrical stimulator (Chattanooga 2-channel Mobile stim) delivered biphasics symmetric rectangular pulses (frec. 25-80 Hz)). The stimulus output is interrupted every $10 \mathrm{~ms}$ to create "bursts" of stimulation every second. The $10 \mathrm{~ms}$ off period was not detectable by the subject. The total time of single procedure was $15 \mathrm{~min}$. Quadriceps was stimulated in extention. On the left quadriceps of the subjects the Interventions using the Kinesiology tape (Kinesiotex gold, Kinesiotaping) were applied by ocupational therapist who has KT certification. The Kinesiology tape was applied with approximately $40 \%$ stretch of its maximal length on the rectus femoris muscle to the superior border of the patella. Both procedures (NMES/KT) were repeated daily for ten days. The electromyographic signal was registered by a dual-channel sEMG Biopac Systems, Inc. ${ }^{\circledR}$ device integrated with computer software for digital analysis and report creation. We realizaed two registrers: before and 10 days after NMES/KT interventions. There were significant differences in resting sEMG activity between inicial and final evaluations for the NMES of all participants (p 0.05) Mean Values of resting EMG activity for the basketball players/ sedentary groups was increased by 90 and $152.3 \%$ respectively while in maximum contraction was 39.8 and $38.36 \%$. There were no significant differences in resting and maximum contraction sEMG activity between initial and final observations for the KT for all participants.

Kinesiology taping, Neuromuscular electrical stimulation, Electromyography, functional performance, Quadriceps Femoris
$\mathrm{P} 2-40$

FAT OXIDATION RATE RELATED TO PLASMA LIPIDS RESPONSE IN INCREMENTAL EXERCISE

\author{
Marisol Soria ${ }^{(1)}$, Miguel Anson ${ }^{(2)}$, Manuel Guerra ${ }^{(3)}$, Jesus \\ Escanero ${ }^{(3)}$ \\ (1) Facultad Ciencias de la salud y del deporte, Huesca, Spain; \\ (2) Hospital Clinico, Zaragoza, Spain; ${ }^{(3)}$ Facultad de Medicina, \\ Zaragoza, Spain
}

Fat and carbohydrate $(\mathrm{CHO})$ are the major substrates in skeletal muscle for energy production during exercise. The exercise intensity that causes the maximal fat oxidation (MFO) rate is

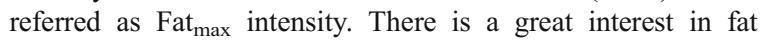
metabolism and its regulation because It has been suggested that training at Fat ${ }_{\max }$ intensity is recommended to maximize the beneficial effects of exercise and may have utility for endurance performance. The aim of this study was to investigate the relationship among fat oxidation rate and fat max zone and plasma lipids concentrations in well-trained athletes. A total of twenty-five trained triathletes completed a graded cycleergometer test to exhaustion with increasing by $0,5 \mathrm{~W} \cdot \mathrm{Kg}^{-1}$ every $10 \mathrm{~min}$. Fat oxidation rates were determined using indirect calorimetry. The intensity which elicited maximal fat oxidation (Fat $\max _{\text {a }}$ was found at $59,3 \pm 4,1 \% \mathrm{VO} 2_{\max }$ and the intensity at which fat oxidation became negligible (Fat min $)$ was found at a relative work intensity of $88,4 \pm-7,2 \% \mathrm{VO} 2_{\text {max }}$. Fat max zone were calculed around $\pm 20 \%$ of Fat max. Blood samples for lipids analysis were collected at the end of each stage of the graded exercise test. All variables studied showed an increase at the end of incremental protocol respect basal levels.

In Fat max zone free fatty acids (FFA) variations not showed significant because there increase observed between values at $-20 \%$ of Fat max intensity and Fat max return to the same level of the initial. Triglycerides (TG), CHOL total, LDL, HDL, increased significantly along Fat max zone. These results suggest that exercise intensity is related to plasma lipids levels. At intensities around fat max $( \pm 20 \%)$ plasma FFA variation differs from other lipids. These results may have application for the more adequate exercise intensity prescription to maximize the beneficial effects of exercise.

\section{P2-41}

RELATION BETWEEN PLASMA PROLACTIN CONCENTRATION AND PLASMA OSMOLALITY IN EUHYDRATED ATHLETES

Marisol Soria (1), Katty Espinosa ${ }^{(2)}$, Mercedes Zaragueta ${ }^{(2)}$, Miguel Anson (3), Jesus Escanero ${ }^{(2)}$

(1) Facultad de Ciencias de la Salud y deporte, Huesca, Spain; (2) Facultad de Medicina, Spain; ${ }^{(3)}$ Hospital Clinico, Zaragoza, Spain

Exercise represents a type of stress condition to the body that can affect neuroendocrine function. An often reproducible finding is an increase in PRL secretion. It is known that exercise stimulates the release of the hormone prolactin (PRL) depending on the 
intensity and duration. After cessation of acute exercise this effect persists and continues during the recovery period. It remains unknown whether exercise induced PRL secretion. The variations of PRL have been related to metabolic disturbances occurring during exercise: changes in osmolality, blood volume or lactate concentration.

The aim of this study was to investigate the relationship among plasma prolactin variation, plasma osmolality and plasma ions concentrations in well-trained and euhydrated athletes during incremental exercise. A total of twenty-five trained triathletes completed a graded cycle-ergometer test to exhaustion with increasing by $0,5 \mathrm{~W} \cdot \mathrm{Kg}^{-1}$ every $10 \mathrm{~min}$. Blood samples for lipids analysis were collected at the end of each stage of the graded exercise test. All variables studied showed an increase at the end of incremental protocol respect basal levels.

Plasma prolactin levels showed a progressive increment between basal and the end of exercise protocol. This increment becomes significant when intensity of work-load was $>80 \%$ VO2. Plasma osmolality increased along the exercise between $287 \mathrm{mOsm} \mathrm{L}^{-1}$ until $300 \mathrm{mOsm} \mathrm{L}^{-1}$ at the end of the exercise $(p<0,05)$. Plasma sodium $(\mathrm{Na})$, chloride $(\mathrm{Cl})$ and potassium $(\mathrm{K})$ concentrations increased significantly along exercise but these increases were different for each ion. Plasma $\mathrm{Na}$ values increased only at the end of incremental exercise ( $>80 \% \mathrm{VO} 2$ max) while plasma $\mathrm{Cl}$ increases along the exercise protocol. Plasma PRL variation showed no correlation whit plasma osmolality or plasma $\mathrm{Na}$ or $\mathrm{K}$ levels. Plasma PRL was correlated with Plasma $\mathrm{Cl}$ variation. Positive correlation was found between osmolality and plasma $\mathrm{Na}$ concentration.

These results suggest that exercise intensity is related to plasma PRL variation in incremental exercise. The association between plasma PRL and plasma $\mathrm{Cl}$ concentrations suggest a relation between plasma PRL increase and hyperchloraemia.

\section{P3-01}

IMPAIRED HEMOSTASIS IN HEREDITARY HEMORRHAGIC TELANGIECTASIA MICE MODEL

Cristina Egido Turrión, Claudia Ollauri Ibáñez, Laura Ruiz Remolina, José Miguel López Novoa, Miguel Pericacho

Departamento Fisiología y Farmacología. Universidad de Salamanca, Salamanca, Spain

Hereditary Hemorrhagic Telangiectasia (HHT) is a rare disease caused by mutations in ENG (endoglin) and ACVRL1 (ALK1) genes in $90 \%$ of cases. HHT is characterized by the presence of telangiectasias and arteriovenous malformations (AVMs). Telangiectasias and AVMs rupture causes severe and difficult to stop bleedings that compromises patients' life, causing death in one out of three HHT patients. Hemorrhage frequency and intensity leads to periodical transfusion needed, depleting patient's life quality and causing large healthcare spending. It is considered that HHT is caused by an impaired angiogenesis that leads to the formation of fragile and immature vessels. However, our hypothesis propose that frequent hemorrhages would be explain by the rupture of malformation of blood vessels, while the severity of the bleedings could be consequence of an impaired hemostasis.
Previous results from our group demonstrate that endothelial endoglin RGD domain interacts with integrins of leukocytes. This suggest that endothelial endoglin could also interact with platelet integrins having a role in hemostasis. To assess this hypothesis, we analyze different components of hemostasis in a HHT animal model, endoglin heterozygous mice $\left(\mathrm{Eng}^{+/}\right)$.

Our results prove that bleeding time is increased in endoglin deficient mice compared with WT. This alteration seems not to be due to an impaired platelet activation or aggregation, since there are no differences in these processes between platelets obtained from $\mathrm{Eng}^{+/+}$or $\mathrm{Eng}^{+/-}$mice. Moreover, we analyze in vivo thrombogenesis injecting a sub-lethal mixture of collagen and epinephrine, and no differences were obtained in heterozygous mice compared with control animals. Finally, we induce closure of carotid artery by the action of ferric chloride placed on the surface of exposed carotid. Data acquired shows that thrombus stabilization is decreased in $\mathrm{Eng}^{+/-}$ mice, whose capacity to form thrombus able to occlude the vessel are diminished. Thus, we can conclude that $\mathrm{Eng}^{+/-}$mice have a lower capacity of hemostasis. We suggest that this issue is not due to problems in platelet function but an impaired interaction between platelets and endothelial endoglin trigger defective thrombus stabilization. These could represent a great progress in the knowledge of hemorrhage etiopatogenia of HHT patients.

Hereditary Hemorrhagic Telangiectasia, HHT, Endoglin, ALK1, Hemostasis

P3-02

METABOLIC SYNDROME: BLOOD PRESSURE DURING PREGNANCY AND LACTATION. GESTATIONAL PARAMETERS IN OFFSPRING

Alejandra Serrano, Paula Sobrino Calero, María Luisa Ojeda Murillo, Fátima Nogales Bueno, Olimpia Carreras Sánchez

Departamento de Fisiología. Facultad de Farmacia, Sevilla, Spain

Objective: The aim of this study is to analyze blood pressure (BP) levels in dams exposed to metabolic syndrome in both gestation and lactation periods. In order to evaluate if these effects are dangerous to offspring, gestation and lactation parameters will also be analyzed.

Material and methods: Male and female Wistar rats were randomized into two experimental groups: Control group fed with basic diet and Metabolic Syndrome group fed with a high-fructose diet $(65 \%)$. These treatments lasted 9 weeks (3 induction weeks, 3 pregnancy weeks and 3 lactation weeks). The tail occlusion method was employed to measure blood pressure and heart rate using the meter NIPREM 645, cylindrical traps, infrared light lamp and a computer program. Gestational and lactation indexes were also calculated.

Results: At the end of pregnancy, metabolic syndrome dams' presented increased systolic BP and decreased diastolic BP values; heart rate values were unaltered. However, at the end of lactation, these rats had increased systolic and diastolic BP values; heart rate values were decreased. Fructose exposed 
offspring had higher birth weight than control rats, but at the end of lactation, they weighed less than control rats. Despite gestational index were similar in both groups, lactation survival index was significantly decreased in fructose exposed rats. Moreover, fructose exposed lactating pups had cardiomegaly and a low urinary flow.

Conclusion: These results show that metabolic syndrome has cardiovascular effects in dams, and that these effects are more pronounced during lactation period. These disorders seem to affect to their offspring especially during lactation.

Metabolic Syndrome, Pregnancy, Lactation, Blood Pressure

P3-03

“BINGE DRINKING" AFFECTS THE AMOUNT OF SELENIUM BIOLOGICALLY ACTIVE IN THE TISSUES. SUPPLEMENTATION AS THERAPY

Paula Sobrino Calero, Alejandra Serrano Ruiz, Maria Luisa Ojeda Murillo, Fatima Nogales Bueno, Olimpia Carreras Sanchez

Departamento de Fisiologia. Facultad de Farmacia. Univ. Sevill, Sevilla, Spain

Introduction: Alcohol consumption type "binge drinking" (BD) causes cardiovascular alteration and oxidative stress. Selenium (Se) is a essential micronutrient, present in many foods with an important role as an antioxidant, being part of the antioxidant enzyme glutathione peroxidase (GPx), which slows the oxidative damage caused by alcohol.

Objectives: Analyze how "binge drinking" affects absorption, retention and tissue distribution of Se in the organism as well as the antioxidant activity of selenoprotein GPx in the tissues.

Methods: It was used teenagers rats divided in four groups: control, selenium control, alcohol, selenium alcohol. Alcohol was administered intraperitoneally ( $3 \mathrm{~g} / \mathrm{kg} /$ day) and in the supplemented groups selenium was administered as sodium selenite $(0.14 \mathrm{ppm})$ in the drinking water. SE levels were determinated by atomic absorption spectrophotometry while GPx activity was analyzed by spectrophotometry UV / Vis in the liver, heart and kidney.

Results: The apparent absorption and retention of Se is not affected by alcohol. The supplementation increases retention of Se. Nevertheless, tissue deposits of Se in liver, heart and kidney, as well as in serum are decreased in the alcohol group. Supplementation with Se increased these deposits. Similarly, the activity of the antioxidant selenoprotein GPx diminished after alcohol consumption and increased after supplementation with Se.

Conclusions: "Binge drinking" type of alcohol consumption alters homeostasis of $\mathrm{Se}$ in the organism. After analyzing the results it can be appreciated a possible homeostatic attempt by the body to retain $\mathrm{Se}$ in alcohols groups.

Supplementation with Se of the diet could be a good therapy to increase the levels of Se in serum, liver, heart and kidney and the antioxidant activity of the enzyme GPx in the organs studied, minimizing the oxidants effects of alcohol in these tissues.

Binge drinking, selenium supplementation, selenium homeostasis

P3-04

ORAI1 AND TRPC1 INTERACT WITH CA 1.2 L-TYPE CA $^{2+}$ CHANNELS IN MOUSE AND RAT VASCULAR SMOOTH MUSCLE CELLS

Javier Ávila-Medina ${ }^{(1)}$, Eva Calderón-Sánchez ${ }^{(1)}$, Paula CallejoGarcía $^{(1)}$, Juan Antonio Rosado ${ }^{(2)}$, Antonio Ordóñez-Fernández (1), Tarik Smani ${ }^{(1)}$

(1) Group of Cardiovascular Phisiopathology, Department of Medical Physiology and Biophysics, Institute of Biomedicine of Seville, Hospital of Virgen del Rocío/CSIC/University of Seville, Sevilla, Spain; ${ }^{(2)}$ Departament of Physiology, University of Extremadura, Cáceres, Spain

Objetives: Vasoactive agonists regulate vascular tone by an increase of the intracellular calcium concentration in vascular smooth muscle cells (VSMCs), due to a rapid calcium release from sarcoplasmic reticulum and later through calcium influx from extracellular medium via voltagedependent calcium channels (VDCC), particularly by $\mathrm{Ca}_{\mathrm{V}} 1.2$ L-type calcium channel (LTCC), and/or voltageindependent channels including store operated calcium channels (SOCC).

Evidences suggested that Orail and TRPC1 form SOCC in VSMCs. Here we studied the distribution of these two proteins and their functional interaction with $\mathrm{Ca}_{\mathrm{V}} 1.2$ LTCC in VSMCs in vascular tone regulation.

Materials and methods: Organ chamber was used to study the contraction in mouse aorta and rat coronary artery. Protein distribution was assessed by in situ Proximity Ligation Assay (PLA) in freshly isolated VSMCs.

Results: Serotonin (5-HT) or endothelin-1 (ET-1), stimulate vasoconstriction involving calcium entry through SOCC and LTCC in aorta and coronary arteries. The induced vasoconstriction was sensitive to widely used inhibitors of LTCC and SOCC. PLA immunofluorescence experiments determined that under basal conditions, Orail and TRPC1 colocalize separately with $\mathrm{Ca}_{\mathrm{V}} 1.2$ and share the same subcellular microdomains in aortic and coronary VSMCs. Interestingly, upon agonists treatment Orail and TRPC1 enhance their interaction with Cav1.2 in VSMCs.

Conclusions: Our data suggest that vasoactive agonists promote a vessel contraction as a result of a co-activation of both $\mathrm{Ca}_{\mathrm{V}}$ 1.2-dependent LTCC and SOCC channels, favoured by their functional interaction.

Supported by Spanish Ministry of Economy and Competitiveness (BFU2013-45564-C2); and by Andalucia Government (P10-CVI6095).

Orai1, TRPC1, CaV1.2, vascular tone regulation 
ELECTROPHYSIOLOGICAL EFFECTS OF THE NO DONOR S-NITROSOGLUTATHION DURING MYOCARDIAL ACUTE LOCAL STRETCH

Luis Such-Miquel $^{(1)}$, Irene Del Canto ${ }^{(2)}$, Carlos Soler ${ }^{(1)}$, Manuel Zarzoso ${ }^{(1)}$, Laia Brines ${ }^{(2)}$, Óscar Julián Arias-Mutis ${ }^{(2)}$, Patricia Genovés ${ }^{(2)}$, Germán Parra ${ }^{(1)}$, Antonio Manuel Alberola ${ }^{(1)}$, Luis Such $^{(1)}$, Francisco Javier Chorro ${ }^{(1)}$

${ }^{(1)}$ Universitat de València (E. G.), Valencia, Spain; ${ }^{(2)}$ Fundación de Investigación, Hospital Clínic Universitari de Valencia (INCLIVA), Valencia, Spain

Introduction: Stretching causes changes in responses of cell membranes. Some are carried out by effect of nitric oxide (NO) released after stretching when activating, among others, mechanosensitive ion channels. Moreover, ventricular fibrillation (VF) is produced by tissue heterogeneities from intracardiac pressures, by destructuring the walls or for example, by damage in postinfarction scarring. We wanted to try to unravel one of the mechanisms after the generation of local stretch, present in pathologies that cause heterogeneities. The analysis of these heterogeneities, interpreting the characteristics of complexity of the electrical activation in epicardia during VF (CEVF), can help to predict the difficulty in defibrillating and the recovery of cardiac mechanics, and it allows to describe effects of various factors involved in VF. The markers of the degree of CEVF could be useful to characterize the effects of various aspects on the fibrillation processes for an effective defibrillation. We have used a NO donor, the SNitrosoglutathione (GSNO), to investigate changes in the CEVF in an isolated heart, after local stretching.

Methods: Hearts from male NZW rabbits (10 controls, 11 treated with GSNO 10micromolar, and 10 with GSNO 50micromolar) were used. After euthanasia (according ethical recommendations) and heparinization, the hearts were placed in a Langendorff setup. A stimulation electrode and a recording multielectrode were placed on left ventricular epicardium. VF was caused by overstimulation. After $5 \mathrm{~min}$ of $\mathrm{VF}$ (with a nonstop coronary perfusion), stretching was induced with a device into the left ventricle throughout the left atrium. Measurements were made 1 min before and $3 \mathrm{~min}$ after the onset of the stretch. After drawing the electrical activation epicardia maps, an index of complexity (IC) was calculated $=[($ number of type I maps $x 0.1)+($ number of type II maps $\mathrm{x} 1)+($ number of type III maps $x 2)]$ / number of analyzed maps. Type I: simple wave fronts without blocking lines or areas of slow conduction. Type II: two wavefronts, or one with areas of conduction block or slow driving. Type III: 3 or more wavefronts associated with blocking areas and slow conduction. Differences were accepted when $p<0.05$.

Results: In Control group, stretching caused an increase in the complexity $(1.27 \pm 0.13$ vs $1.60 \pm 0.15)$, as well as in the GSNO 10 micromolar group $(1.32 \pm 0.15$ vs $1.61 \pm 0.15)$. Nevertheless, no differences were observed in GSNO 50micromolar group when stretching $(1.77 \pm 0.18$ vs $1.84 \pm 0.17)$.

Conclusion: The nitric oxide donor S-Nitrosoglutathion, showed a protective effect against the heterogeneities due to stretching at the highest concentration used.

Stretch, heart, electrophysiology, nitric oxide, GSNO
EFFECTS OF THE NITRIC OXIDE DONOR SNITROSOGLUTATHION AND ACUTE LOCAL VENTRICLE STRETCHING ON REFRACTORINESS AND ELECTROPHYSIOLOGICAL HETEROGENEITY

Luis Such-Miquel $^{(1)}$, Irene Del Canto ${ }^{(2)}$, Carlos Soler ${ }^{(1)}$, Manuel Zarzoso $^{(1)}$, Laia Brines ${ }^{(2)}$, Óscar Julián Arias-Mutis ${ }^{(2)}$, Patricia Genovés ${ }^{(2)}$, Germán Parra ${ }^{(1)}$, Antonio Alberola ${ }^{(1)}$, Luis Such ${ }^{(1)}$, Francisco Javier Chorro ${ }^{(1)}$

(1) Universitat de València (E. G.), Valencia, Spain; ${ }^{(2)}$ Fundación de Investigación, Hospital Clínic Universitari de Valencia (INCLIVA), Valencia, Spain

Introduction: Refractoriness and heterogeneity of the electrical activation are related to the genesis and maintenance of reentry responsible for arrhythmias such as ventricular fibrillation (VF). Stretching produces acute electrophysiological effects as shortening of refractoriness and increased myocardial electrophysiological heterogeneity. Nitric oxide (NO) modulates inotropic responses caused by stretching and transmembrane currents. Other electrical responses are not so well-known. We investigated two concentrations of the NO donor S-Nitrosoglutathione (GSNO), in order to analyze changes in the spectral characteristics of VF: dominant frequency (DFr) and spectral concentration $(\mathrm{SpC})$, produced by ventricular acute local stretching during the arrhythmia.

Methods: We used 31 hearts from White New Zealand rabbits (10 in Control group, 11 treated with GSNO 10 micromolar and 10 treated with GSNO 50 micromolar). After heparinization and euthanasia (according European ethical laws), their hearts were removed and placed in a Langendorff setup. A stimulation electrode and a recording multielectrode were placed in left ventricular epicardium. VF was induced stimulating at increasing frequencies keeping coronary perfusion. After $5 \mathrm{~min}$ of VF, the left ventricle was stretched from inside using a device introduced through the left atrium. Measurements were made 1 , and $3 \mathrm{~min}$ before and after the onset of stretching.

Results: There were no differences in the percentage of increase in the DFr during stretching. When comparing with the Control group $(47.1 \%)$ the percentage was lower in the 10 micromolar group $(23.3 \%)$, while there were no differences when comparing with 50 micromolar group $(28 \%)$ at baseline. There was a decrease in the percentage of $\mathrm{SpC}$ after stretching in the 10 micromolar group (33.4\% vs 18.6 ; $p<0.05)$. No significant differences were found when comparing with the 50micromolar group (29\%).

Conclusions: The nitric oxide donor S-Nitrosoglutathione (10 micromolar), reduces the effects of ventricular acute local stretching on the dominant frequency of ventricular fibrillation (as an index of refractoriness) and spectral concentration (as an index of heterogeneity), indicating a beneficial effect.

Stretch, heart, refractoriness, heterogeneity, nitric oxide, GSNO 
HYPOXIA AND COLD EFFECTS ON MYOCARDIUM, DIAPHRAGM AND HEMATOLOGICAL PARAMETERS OF LABORATORY RATS

Cristian Arvey Guzmán David ${ }^{(1)}$, Ana Maria Ortega Hernández ${ }^{(1)}$, Deisy Carolina Ortiz González ${ }^{(1)}$, Garoa Santocildes ${ }^{(2)}$, Teresa Pagès ${ }^{(2)}$, David Rizo Roca ${ }^{(2)}$, Joan Ramon Torrella ${ }^{(2)}$, Ginés Viscor ${ }^{(2)}$

(1) Universidad Nacional de Colombia, Bogotá, Colombia;

${ }^{(2)}$ Universitat de Barcelona, Barcelona, Spain

Introduction: Acclimation to hypoxia and cold involves different responses at physiological level. Both conditions affect $\mathrm{O}_{2}$ homeostasis due to shortages caused by hypoxia and increased coldinduced metabolic rate. This suppose changes in systems of uptake, distribution and oxygen delivery. Our goal was to analyze the effects of combined intermittent exposure to cold and hypobaric hypoxia on the heart, diaphragm muscle and some hematological parameters of rats.

Methods: Male Sprague-Dawley rats were randomly assigned to two groups: hypoxia + cold (COHY) and control (CTRL). The COHY group was subjected to $4 \mathrm{~h}$ daily sessions of intermittent hypobaric hypoxia into a refrigerated $\left(6^{\circ} \mathrm{C}\right)$ hypobaric chamber 6 days by week for 21 days at a simulated altitude of $4500 \mathrm{~m}$. At the end of the protocol, we extracted a blood sample, and tibialis anterior, gastrocnemius, diaphragm muscles, the heart and the perigonadal white and brown adipose tissues were obtained. Organs were freshly weighted and relative to body mass ratios were calculated. Blood counts and hematimetric parameters were obtained.

Results: No significant differences were observed in body weight between animals of CTRL and COHY groups, but significant differences $(P<0.05)$ were detected in the relative weights (organ weight/body weight) of heart (CTRL $3.12 \pm 0.20 \mathrm{vs.} \mathrm{COHY} 3.50$ $\pm 0.16 \mathrm{mg} / \mathrm{g}$ ), diaphragm (CTRL $1.92 \pm 0.24$ vs. COHY 2.46 $\pm 0.36 \mathrm{mg} / \mathrm{g}$ ), white adipose tissue (CTRL $9.78 \pm 0.75$ vs. COHY $11,25 \pm 0,80 \mathrm{mg} / \mathrm{g}$ ) and brown adipose tissue (CTRL $1.11 \pm 0.13$ vs. COHY $1.33 \pm 0.14 \mathrm{mg} / \mathrm{g})$. Significant differences $(P<0.001)$ were also observed for: RBC count (CTRL $6.60 \pm 0.43$ vs. COHY $8.65 \pm 0.2510^{6} \cdot \mu \mathrm{L}^{-1}$ ), hemoglobin concentration (CTRL 14.0 \pm 0.9 vs. COHY $18.9 \pm 0.6 \mathrm{~g} \cdot \mathrm{dL}^{-1}$ ), hematocrit (CTRL $36.4 \pm 2.5$ vs. COHY $48.1 \pm 1.3 \%$ ) and MCHC (CTRL $38.5 \pm 0.3$ vs. COHY $\left.39.3 \pm 0.6 \mathrm{~g} \cdot \mathrm{dL}^{-1}\right)$.

Conclusions: Cold and hypoxia exposure altered distribution and oxygen delivery in order to maintain cellular bioenergetics status. The main physiological adjustments found were: 1) Hypertrophy of the diaphragm, reflecting the respiratory overload caused by hypoxia. 2) Moderate cardiac hypertrophy due to the blood and plasma volume expansion caused by both stimuli and induced by hypoxic pulmonary vasoconstriction. 3) Erythropoietic response was high in animals exposed to hypoxia and cold, thus ensuring an adequate oxygen supply for the increased heat production due to thermoregulation. 4) Moreover, the differences observed in the brown adipose tissue were the result of a physiological adjustment to non-shivering thermogenesis.

Hypoxia, cold, erythropoiesis, heart, diaphragm muscle, adipose tissue
THE AKUREYRI (AEY) PERFORMANCE SCORE FOR LABORATORY RATS RUNNING ON TREADMILL

Juan Gabriel Ríos-Kristjánsson ${ }^{(1)}$, Karen Mist Kristjánsdóttir ${ }^{(2)}$, David Rizo Roca ${ }^{(1)}$, Cristian Núñez Espinosa ${ }^{(3)}$, Joan Ramon Torrella $^{(1)}$, Teresa Pagès ${ }^{(1)}$, Ginés Viscor ${ }^{(1)}$

(1) Universitat de Barcelona, Barcelona, Spain; ${ }^{(2)}$ Aarhus University, Aarhus, Denmark; ${ }^{(3)}$ Universidad de Magallanes, Punta Arenas, Chile

Introduction: Although a lot of research has been carried out in exercise physiology with rats running on a treadmill, the particular characteristics of individual running style and physical performance of rats have never been considered closely. It seems clear that not all rats (just as not all humans) are equally good runners.

Methods: The score developed and presented in this study derived as a secondary observational registry from experimental rats running on a treadmill used in a wider research project, the primary experiment, approved by the Experimental Animal Ethics Committee of the University of Barcelona. During the course of 2.5 years, the primary experiment involved 130 male Sprague Dawley rats training on a treadmill to study the recovery of induced skeletal muscle damage in the hind limbs. The rats' exercise regimen was divided into several parts and took 4-7 weeks depending on to which experimental group each rat was assigned. The animals received humane treatment fulfilling the Spanish and European directives for the care of animal uses for scientific purposes.

Results: The score is based on direct observation of the running characteristics of each animal, according to 3 registry criteria, each giving their own rank. These are identified by the letters A, E and Y; which coincides with the IATA airport code for the Icelandic town of Akureyri (AEY) from where the two developmental experimenters come. The criteria for the A-score concerns the physical positioning and actions of the rat whilst running (or avoiding to run). The E-score concerns the position of the rat's tail, specifically, as an indicator of how tired a rat is based on its effort to hold it up (to avoid receiving an electric shock from the grid). The Y-score addresses artefacts and potential problems considering the digital representation of the electric shock count.

Conclusions: Without any new special equipment or expensive investments, we propose a new performance score (AEY) allowing to distinguish between good and bad runners, as a useful way to increase the accuracy of the runningtraining of rats which can reduce the number of required animals. We found noticeable differences along a training program, thus providing evidence of sufficient sensitivity of the AEY score to follow-up the performance improvement in exercise capacity due to training. In addition, we provide a new tool that can be correlated with the variations in other physiological or metabolic parameters.

Laboratory rats, performance, treadmill running, exercise, training 
P3-09

WILD OLIVE OIL INTAKE REDUCES BLOOD PRESSURE AND IMPROVES ANTIOXIDANT CAPACITY AND ENDOTHELIAL FUNCTION IN NITRIC OXIDE-DEPLETED HYPERTENSIVE RATS

Alfonso Mate ${ }^{(1)}$, María Victoria Ruiz-Armenta ${ }^{(2)}$, Gema AguilarEspejo ${ }^{(2)}$, Claudia Reyes-Goya ${ }^{(2)}$, José Luis Miguel-Carrasco ${ }^{(2)}$, María Del Carmen Pérez-Camino ${ }^{(3)}$, Estefanía Burgos-Morón ${ }^{(4)}$, Miguel López-Lázaro ${ }^{(4)}$, Carmen M Vázquez ${ }^{(1)}$

(1) Departamento de Fisiología, Facultad de Farmacia, Universidad de Sevilla. Sevilla, Spain. - Instituto de Biomedicina de Sevilla (IBIS). Sevilla, Spain, Sevilla, Spain; ${ }^{(2)}$ Departamento de Fisiología, Facultad de Farmacia, Universidad de Sevilla. Sevilla, Spain., Sevilla, Spain; (3) Instituto de la Grasa, CSIC. Sevilla, Spain., Sevilla, Spain; ${ }^{(4)}$ Departamento de Farmacología. Facultad de Farmacia. Universidad de Sevilla. Sevilla, Spain., Sevilla, Spain

Background: Wild olive tree (Olea europaea, var. sylvestris) produces a fruit called "acebuchina", which is a drupe with less fleshy pulp and smaller size than its cultivated olive tree counterpart has. The oil obtained from wild olives possesses quite peculiar properties, with higher purity and higher quality than standard extra virgin olive oils. Unfortunately, its market prize is also notably more expensive. There is extensive literature regarding the chemical composition, and pharmacological properties, of leaves and olive oils obtained from cultivated olive trees; on the other hand, studies on the chemical constituents and possible beneficial effects of wild olive byproducts are scarce.

Purpose of the study: To characterize the chemical profile of extra virgin oil obtained from wild olives, and to analyze the antioxidant capacity and endothelial function of rats with hypertension induced by chronic administration of $\mathrm{N}(\mathrm{G})$-nitro-L-arginine methyl ester (L-NAME), with or without simultaneous chronic administration of "acebuchina" oil.

Methods: Four groups of male Wistar rats were used: i) rats fed with a standard maintenance diet (AIN-93 M); ii) rats subjected to 12-week treatment with AIN-93 M supplemented with $15 \%$ acebuchina oil; iii) rats fed with standard AIN-93 M diet, and also subjected to 12 -week administration of L-NAME $(20 \mathrm{mg} / \mathrm{kg}$ body weight/day, dissolved in drinking water); and iv) rats subjected to 12-week treatment with both L-NAME and $15 \%$ acebuchina oilenriched AIN-93 M. The chemical composition of wild olive oil was determined by gas chromatography and high performance liquid chromatography. Systolic blood pressure was monitored weekly throughout the experimental period. Upon completion of treatments, blood samples were collected, and the animals were humanely killed for harvesting the thoracic aorta, in order to perform cardiovascular reactivity experiments in aortic ring segments in a compact organ bath filled with Krebs physiological solution. The activities of antioxidant enzymes, glutathione peroxidase, glutathione reductase and superoxide dismutase, were assayed using commercial kits.

Results: High levels of oleic acid and triterpenes, namely maslinic acid and oleanolic acid, as well as erythrodiol, were found in wild olive oil. In addition, acebuchina oil administration resulted in a significant reduction of systolic blood pressure in hypertensive rats. L-NAME-treated rats also presented with alterations in the antioxidant capacity and with endothelial dysfunction, which was ameliorated by simultaneous intake of acebuchina oil.

Conclusions: Antioxidant properties of wild olive oil result in beneficial effects on endothelial function and blood pressure regulation. This might support the use of this product as a preventive agent against cardiovascular disease.

Endothelial dysfunction, L-NAME-induced arterial hypertension, olive oil, oxidative stress, wild olive tree

P3-10

DIFFERENTIAL VULNERABILITY TO GLOBAL CEREBRAL ISCHEMIA. THE ROLE OF THE NEUROVASCULAR UNIT

Berta Anunciabay-Soto, Diego Pérez Rodríguez, María Santos Galdiano, Enrrique Font Belmonte, Irene Fernández Ugidos, Paloma González Rodríguez, José Manuel Gonzalo Orden, Marta Regueiro Purriños, Arsenio Fernández López

Ibiomed, León, Spain

Stroke is one of the main causes of mortality in developed countries. The higher vulnerability to stroke of hippocampus compared to other brain structures is still an unsolved paradigm. In the past, intrinsic properties of the neurons of the nervous system were though to be responsible for this differential vulnerability, but recently it has been proposed the hypothesis that the neurovascular unit (endothelial cells, pericytes and glial cells) are also involved in this issue. The present study analyzes this hypothesis using a model of global cerebral ischemia in rat, which was carried out by clamping for $15 \mathrm{~min}$ both common carotid arteries with moderate hypotension. Three different reperfusion times ( $24 \mathrm{~h}, 48 \mathrm{~h}$ and 7 days) were analyzed and the response in three brain structures reported to present different ischemia vulnerability (hippocampal CA1 and CA 3 areas as well as the cerebral cortex above them) was compared. The total number of neurons was estimated by Nissl staining to assess ischemia induced cell mortality. The endoplasmic reticulum stress and the auophagy pathway was analyzed by qPCR, Western blot and immunofluorescence assays. To elucidate the role of the neurovascular unit, parameters of immflamation, gliosis, leukocyte migration and metalloproteases were quantified. After ischemia, differences between the three brain structures were observed, not only in the neural homeostatic response but also in the neurovascular unit, which indicates that this latter is also involved in the differential vulnerability to ischemia. This study was granted by MINECO (reference BIO2013-49006C2-2-R), which also supports Berta Anuncibay-Soto and María Santos-Galdiano by fellowships. Diego Pérez Rodríguez and Irene Fernández Unidos have grants from Junta de Castilla y León (EDU/346/2013).

Global ischemia, Cell homeostasis, ER-stress, Autophagy, Neurovascular unit 
P3-11

DAB-2 PROTEIN REGULATES APICAL JUNCTION PROTEINS IN CACO-2 CELLS

$\mathrm{M}^{\mathrm{a}}$ Dolores Vazquez-Carretero ${ }^{(1)}$, Pablo García-Miranda ${ }^{(1)}$, José Manuel Serrano-Morales ${ }^{(1)}, \mathrm{M}^{\mathrm{a}}$ José Peral $^{(1)}$, A. Ana Ilundáin ${ }^{(1)}$, Karl Matter $^{(2)}$, M ${ }^{\text {a }}$ Susana Balda ${ }^{(2)}$

(1) Department of Physiology. University of Seville, Sevilla, Spain;

(2) Department of Cell Biology, Institute of Ophthalmology, University College London., London, United Kingdom

Dab2 is an intracellular adaptor protein involved in clathrin mediated endocytosis in the kidney, visceral endoderm and intestine ${ }^{1}$. We previously proposed that Dab2 mediates the endocytosis of milk macromolecules in the intestine of suckling rats ${ }^{2,3}$ and in the current study we investigate whether it mediates the endocytosis of components of the intestinal epithelium's intercellular junctions. For this, Dab2 gene was silenced in human cultured cells, derived from either epithelial colorectal adenocarcinoma cells (Caco-2) or dermal microvascular EC cells (HDMEC), by RNA-mediated interference experiments using Dab2 targeting siRNAs and nontargeting siRNAs (control). Location and abundance of proteins of tight, adherens and focal junctions were evaluated by immunofluorescence and western-blot assays, respectively. The functionality of the tight junctions was tested by determining paracellular permeability to ions and to hydrophilic tracers by measuring the transepithelial electrical resistance (TER) and transmonolayer flux of fluorescent dextrans, respectively. Silencing Dab2 protein alters the distribution of tight and adherens junction components, but not of proteins of focal adhesions in both cell types. Though the location of the proteins is modified by Dab2 depletion neither their expression, nor the monolayer permeability is affected by Dab2 silencing. Our data thus suggest that Dab2 has a role on junction stability. In conclusion, the results show for the first time that the depletion of Dab2 leads to a disruption of tight and adherens junctions, suggesting the participation of Dab2 in the transport of proteins to and from the cell junctions by acting as an adapter protein in endocytic/exocytic processes. (EMBO short term fellowship ASTF 45-2012)

1. Madden and Swiatecka-Urban (2012) Commun Integr Biol 5:473-476.

2. Vázquez-Carretero et al. (2011) J Cell Biochem 112:354-361.

3. Vázquez-Carretero et al. (2014) J Cell Biochem 115:510-522.

Dab2, caco-2, intercellular junctions

P3-12

STIMULATION OF PHAGOCYTOSIS AND MICROBICIDAL ACTIVITY OF PERIPHERAL GRANULOCYTES BY THE BIOREGULATORY MEDICAMENT ENGYSTOL. ROLE OF TLR2

Leticia Martín-Cordero $^{(1)}$, Juan Luis Quevedo ${ }^{(2)}$, María Dolores Hinchado ${ }^{(3)}$, Pedro Manonelles ${ }^{(2)}$, Eduardo Ortega ${ }^{(3)}$
(1) Dpto. Enfermería (Grupo Inmunofisiología). C.U.P. UEx, Plasencia, Spain; ${ }^{(2)}$ Universidad Católica San Antonio de Murcia, Murcia, Spain; ${ }^{(3)}$ Dpto. Fisiología (Grupo Inmunofisiología). Facultad de Ciencias. UEx, Badajoz, Spain

In the context of complementary "bioregulatory medicine", which is intended to naturally stimulate the innate host defences, it is crucial to explore how the innate immune system is affected by multi-target combinations in order to demonstrate the scientific rational underlying the benefits frequently reported in clinical practice. Engystol (Heel GmbH, Baden-Baden, Germany) is a multi-target combination (containing Vicetoxicum hirundinaria and sulphur) commonly used for the profilaxis and treatment of infectious diseases. Previous clinical studies have demonstrated good tolerability, efficacy and safety, particularly in respiratory tract infections. Neutrophils (the main circulating innate immune cells) constitute the first line of defence against pathogens that have overcome the organism's "natural barriers". The objective of the present study was to investigate a potential mechanism regulating the effectiveness of Engystol through stimulation of the phagocytic and microbicidal capacity of granulocytes; as well as a potential role of Toll-like receptor (TLR) 2, which is the most important TLR present on the surface of human neutrophils for mediating the stimulation of their phagocytic activity. Methods: The phagocytosis and microbicidal activity of opsonised bacteria in response to Engystol were evaluated in whole blood from human volunteers by flow cytometry. Prior to phagocytosis and oxidative burst assay, blood from each donor was incubated with Engystol (or the same volume of $0.9 \% \mathrm{ClNa}$ as vehicle) during $1 \mathrm{~h}$. To determine a potential role of TLR2, assays involving the preincubation $(10 \mathrm{~min}$ ) of blood with anti-TLR2 prior addition of Engystol were included in the experiment. Results: Engystol was found to stimulate the phagocytosis and microbicidal activity of granulocytes. Additionally, TLR2 seems to be involved in the effects of Engystol on phagocytosis because the pre-incubation of whole blood with anti-TLR2 abolished the Engystol-induced stimulation of phagocytic activity. Conclusion: Engystol stimulates the phagocytic and microbicidal activities mediated by granulocytes, which are essential in host defences to combat infectious pathogens. These results provide a potential mechanism for the previously reported benefits of this medicament observed in clinical studies and could also contribute to our understanding of the mechanisms of action of other natural products or multi-target combinations.

This investigation has been partially supported by Gobierno de Extremadura-FEDER (GR 15041) and an agreement UEX-“Laboratorios Heel España S.A.U” (211/14)

Engystol, Granulocytes, Phagocytic microbicidal activity, TLR2

P3-13

\section{EFFECTS OF THE HEAT SHOCK IN THE CHEMOTHERAPY AGENTS-INDUCED APOPTOSIS}

Miriam Rol, Ana María Marchena, Lourdes Franco, Lorena H Martínez, José Antonio Pariente, Ana Beatriz Rodríguez, Ignacio Bejarano

Universidad de Extremadura, Badajoz, Spain 
Facing with cellular stress, HSP are molecular chaperones which prevent denatured protein aggregation accelerating the enzyme refolding, in particular heat stress. HSP are highly expressed in acute myeloid leukaemia cells (AML) as the human U937 cell line. The HSP over-expression in cancer cells confers resistance to apoptosis. Changes in HSP degradation could be an essential parameter to become cancer cells more sensitive, for instance to the apoptosis induced by anticancer drug, however it is poorly studied. For this aim U937 cells or human blood leucocytes were exposed to $42{ }^{\circ} \mathrm{C}$ for $1 \mathrm{~h}$. MTT assay showed a time-decreasing viability of U937 cells after the heat shock in parallel to the decreasing HSP90 levels analyzed by western blot. Moreover, after $2 \mathrm{~h}$ of recovery at $37{ }^{\circ} \mathrm{C}$, U937 were treated with $50 \mu \mathrm{M}$ etoposide or citarabine at increasing time. Chemotherapeutic agents by themselves caused a decrease in MTT activity and an increase in the percentage of U937 cells with apoptotic nucleus. The induced heat shock evoked such a decrease of MTT activity that did not indicate any difference between shocked cells with and without chemotherapeutic agents. On the other hand, fresh leucocytes undergo a protection against etoposide or citarabine when they were previously heat shocked, as indicated by a decrease in the number of cells active caspase- $3^{+}$or LIVE/DEAD fixable aqua dead cell staining. This protection is known as thermotolerance based in the induction of HSP. Given the high levels of HSP in U937 cells, an acute heat stress could overcomes the HSP protection threshold, madding cells either more sensitive to drugs or directly leading them to cell death. On the contrary, normal cells were much more resistant to the same stress, even showing a higher protection to further insults. This study shows HSP as a valuable target to beat the leukaemia resistance preserving at the same time the healthy cells.

Supported by Junta de Extremadura-Fondo Social Europeo de Desarrollo Regional (FEDER) grant IB13072 and GR15051.

HSP, Apoptosis, Leukaemia, Chemotherapy, U93.

P3-14

\section{REELIN EXPRESSION IN COLON CANCER PROGRESSION IN MICE}

José Manuel Serrano Morales, María Dolores Vázquez Carretero, Ana Eloísa Carvajal Vázquez, María Luisa Calonge Castrillo, María José Peral Rubio, Anunciación Ilundáin, Pablo García Miranda

Facultad de Farmacia, Universidad de Sevilla, Sevilla, Spain

Colorectal cancer (CRC) is one of the most common cancers in the developed world and we have reported that reelin expression is downregulated in human CRC (1). This study evaluates reelin and $\alpha$-smooth muscle actin ( $\alpha$-SMA) expression along CRC progression and determines whether the absence of reelin increases the sensibility of the organism to tumour development.

Three month-old reeler and wild-type mice were used. Aberrant Crypt Foci (ACF) were induced by intraperitoneal injection of $10 \mathrm{mg} / \mathrm{kg}$ body weight of azoxymethane (AOM) during 6 weeks and small-sized tumours by the same protocol except that the interval kept after the last AOM dose was 24 weeks instead of 4 . Large-sized tumours were induced by a single AOM dose followed by 7 days without treatment, 4 days with $1 \%$ dextran sodium sulphate (DSS) administration in the drinking water and 14 days with tap water. The DSS/tap water cycle was repeated three times. Control group received injections of phosphate buffer solution and tap water. Animals were humanely handled and sacrificed in accordance with the European Council legislation 86/609/EEC concerning the protection of experimental animals. Tumours and the normal adjacent colon were analysed under light microscope and extracted by microdissection. mRNA was isolated from the tissue and gene expression was analysed using RT-PCR.

Reelin mRNA expression is upregulated in ACF and small-sized tumours compared with control group, without significant changes in $\alpha$-SMA mRNA levels. However, in AOM/DSS tumours, reelin expression is downregulated, and this deactivation is accompanied by a smaller downregulation of $\alpha$-SMA. The number of AOM/DSSinduced tumours, expressed as either total or per colon length, are higher in the colon of reeler mice than in the wild-type mice. The mutation also increases the size of the tumours and tumours larger than $2 \mathrm{~mm}$ are only observed in reeler mice. Reeler mice present a lower survival compared to wild-type mice ( 80 and $100 \%$ respectively).

We conclude that: 1. Reelin expression varies with the development of the tumours. 2. Reeler mice present an increased sensitivity to tumour development, suggesting that reelin might play a protective role in CRC progression. 3. The upregulation of reelin in early CRC stages might be a protective reaction. 4 . The reelin downregulation found in later stages of CRC may be produced by other tumour related factors.

Work supported by a grant from the Junta de Andalucía (CTS 5884).

\section{References}

(1)García-Miranda et al. Genes Nutr 2012;6 Suppl 1:51-81.

Reelin, colon cancer, mice

\section{P3-15}

\section{MEMBRANE ARRANGEMENT OF KV1.3 VIA CAVEOLIN INTERACTIONS}

Jesusa Capera ${ }^{(1)}$, Mireia Pérez-Verdaguer ${ }^{(1)}$, Ramón MartínezMármol (2), Marta Camps ${ }^{(3)}$, Comes Núria ${ }^{(4)}$, Michael M. Tamkun ${ }^{(5)}$, Antonio Felipe ${ }^{(1)}$

(1) Molecular Physiology Laboratory, Departament de Bioquímica i Biomedicina Molecular, Institut de Biomedicina (IBUB), Universitat de Barcelona, 08028 Barcelona, Spain; ${ }^{(2)}$ Clem Jones Centre for Ageing Dementia Research, Queensland Brain Institute, The University of Queensland, Brisbane, Queensland 4072, Australia; ${ }^{(3)}$ Departament de Bioquímica i Biomedicina Molecular, Institut de Biomedicina (IBUB), Universitat de Barcelona, 08028 Barcelona, Spain; ${ }^{(4)}$ Departament de Ciències Fisiologia I, Institut de Neurociències, Universitat de Barcelona, 08036 
Barcelona, Spain; ${ }^{(5)}$ Department of Biomedical Sciences, Colorado State University, Fort Collins, CO 80523, United States

Lipid rafts are specialized membrane microdomains rich in sphingolipids and cholesterol. These assemblies are fluid but more ordered and tightly packed than the surrounding bilayer and have been implicated in the organization of many membrane-associated signaling pathways. The targeting of ion channels to these microdomains has emerged as a crucial mechanism of ion channel localization and function. The voltage gated potassium channel Kv1.3 targets to the immunological synapse (IS), which concentrates lipid rafts, and modulates the physiology of $\mathrm{T}$ lymphocytes. In this study, we demonstrate a central role for caveolin in the trafficking of Kv1.3 to lipid raft microdomains. We identified a Caveolin Binding Domain (CBD) motif, located in the amino terminus of the channel, which is a putative caveolin-interacting domain conserved in the Shaker family. Both Kv1.3 and Kv1.5 target lipid rafts, but only Kv1.3 efficiently interacted with caveolin via the CBD. Such association is essential for the channel localization in the lipid raft domains, such caveolae or IS. Moreover, Kv1.3 behavior and activity was conditioned by the presence of caveolin-1. Therefore, the presence of a CBD near the T1 of Kv1.3 has important functional consequences for Kv1.3 channel physiology.

Supported by MINECO, Spain (BFU2014-54928-R and BFU2015-70067-REDC).

Kv1.3, Caveolin, Protein-protein interactions, Lipid raft targeting

P3-16

NEW GENERATION OF CHEMOTHERAPEUTIC AGENTS, EFFECTS ON U937 CELLS

Lourdes Franco, E Fernández, I Bejarano, J Espino, F Luna, E Viñuelas, AB Rodríguez, JA Pariente

Universidad de Extremadura, Badajoz, Spain

Cisplatin (Cis-Pt) and Paladium (Pt) are ones of the most common anti-cancer agents. It is administrated in chemotherapy triggering programmed cell death on cancer cells. However, treatments with Cis-Pt entail unwanted side effects. Therefore, ongoing researches are focused on designing more tumor cell-selective approaches, being more innocuous for healthy cells. In this way, platinum complexes with heterocycles derivative ligands which contain $\mathrm{N}, \mathrm{N}$ donors, as pyrazole, are of great interest as antitumor agents since they allow maintaining the chemical structure of cisplatin varying the stereochemistry of the ligands attached to the metal ion in which lies the bioactivity of the complex. In this study we aim to evaluate the cytotoxic abilities of compounds like Pt and Pd such as TdTn (2-(3,4-diclorofenil)imin-N-(2-tiazolin-2-il)tiazolidin), PdTdTn (TdTn-palladium(II)) and PtTdTn (TdTn-platinum(II) on human lymphoma cell line U-937.

Cells were incubated with $30 \mu \mathrm{M}$ of these compounds for $24 \mathrm{~h}$. Cell enzyme Caspase- 8 activity was measured by fluorimetric methods using the specific fluorigenic substrate. ROS activity was quantified through CM-H2DCFDA. The chromatin condensation of apoptotic cells was revealed upon vital staining (10 min in the dark) with Hoechst 33342.

Our results show that treatments with PtTdTn and PdTdTn during $24 \mathrm{~h}$, produced an important increase of toxicity in U937 cells. Both compounds induced a rise on percentage of cells showing apoptotic nucleus, caspase 8 enzymatic activity and ROS generation with respect not only to the control values, but also to the CisPt values.

New generation of chemotherapeutic agents shows more efficay than the CisPt at inducing apoptosis in U937 cells.

Supported by Junta de Extremadura-Fondo Social Europeo de Desarrollo Regional (FEDER) grant IB13072 and GR15051.

Cisplatin, Chemotherapy, Pyrazole-based complexes

P3-17

PREDICTIONS FROM AN AGENT-BASED MATHEMATICAL MODEL INDICATE THAT LIPID RAFT SIZE AND LIPID MOBILITY IN NON-RAFT DOMAINS INCREASE DURING NORMAL AGING BUT ARE ACCELERATED IN APP/ PS1 MICE, A MODEL OF ALZHEIMER'S DISEASE

Carla Ferri ${ }^{(1)}$, Guido Santos ${ }^{(2)}$, Néstor Torres ${ }^{(2)}$, Mario Díaz ${ }^{(1)}$

(1) Laboratory of Membrane Physiology and Biophysics, Department of Animal Biology, Universidad de La Laguna. Associate Research Unit ULL- CSIC "Membrane Physiology and Biophysics in Neurodegenerative and Cancer Diseases"., San Cristóbal de La Laguna, Spain; ${ }^{(2)}$ Laboratory of Systems Biology and Mathematical Modelling, Department of Biochemistry, Universidad de La Laguna., San Cristóbal de La Laguna, Spain

Mathematical modeling approaches have recently been used to correlate the effects of lipid composition changes in the physicochemical properties of lipid rafts and non-raft microdomains. In the present study, we have developed an agent based model to assess the effects of normal aging and development of Alzheimer's disease on the evolution of lipid alterations in lipid rafts and nonraft microdomains. We have used experimental data describing the lipid profiles observed in an established model of familial Alzheimer's disease, the APP/PS1 mice model, which we have published recently (Fabelo et al., 2012). We could predict that lipid raft size and lipid mobility in non-raft domains increase during normal aging but these effects are accelerated in the transgenic mouse model. The consequences of these changes are translated to the context of neurotoxic amyloid $\beta$ production. The agent based model predicts that altering membrane microdomain composition (mainly by increasing cholesterol and long-chain polyunsaturated fatty acid docosahexaenoic acid proportions) might delay the onset and progression of Alzheimer's disease.

\section{Reference}

Fabelo, N., Martín, V., Santpere, G., Aso, E., Ferrer, I., and Díaz, M. (2012). Evidence for premature 'Lipid raft aging' in APP/PS1 double transgenic mice, a familial model of Alzheimer's disease. J. 
Neuropathol. Exp. Neurol. 71,858-881.

Alzheimer's disease, lipid rafts, mathematical modeling, agent based model, DHA, cholesterol

P3-18

ARSENITE REGULATES THE EXPRESSION OF PROTEINS INVOLVED IN THE TRANSPORT AND METABOLISM OF ARSENIC SPECIES IN RAT

Cecilia Sosa ${ }^{(1)}$, Natalia Guillén ${ }^{(1)}$, Luis Hortells ${ }^{(1)}$, Cristina Sánchez ${ }^{(2)}$, Victor Sorribas ${ }^{(1)}$

${ }^{(1)}$ Laboratory of Molecular Toxicology, Universidad de Zaragoza, Zaragoza, Spain; ${ }^{(2)}$ Laboratory of Physiology. Universidad de Zaragoza, Zaragoza, Spain

Arsenic is a worldwide environmental pollutant, producing a wide range of adverse health effects. Many studies have focused in the molecular processes by which arsenic promotes disease or in how it is metabolized after absorption, but less is known about the impact on the expression and activity of its transport systems and metabolic routes in vivo.

In this study, the effects of arsenite (As ${ }^{\text {III }}$ ) on the expression of proteins implicated or related to the cellular uptake and efflux of $\mathrm{As}^{\mathrm{III}}$ and arsenate have been analyzed: aquaglyceroporins AQP3/7/9; type II and III phosphate transporters; multidrug resistance protein 2, MRP2, and in the metabolism of arsenical species (arsenite methyltrasferase: AS3MT). Twelve male, two-month old Wistar rats were offered sodium arsenite in drinking water ad libitum: $0 \mathrm{ppb}$ (control group, $n=4), 10 \mathrm{ppb}(n=4)$ and $1000 \mathrm{ppb}(n=4)$. After 10 days of treatment, all rats were sacrificed and tissues were sampled, and frozen immediately. Relative gene expressions were studied by real time PCR.

The RNA expression of $A S 3 M T$ was increased by both concentrations of $\mathrm{As}^{\mathrm{III}}$ in lung, but not in other tissues. Aquaporins constitute an entrance to the cell for $\mathrm{As}^{\mathrm{III}}$. As ${ }^{\mathrm{III}}$ reduced $A Q P 3$ in duodenum, jejunum and kidney, whereas $A Q P 7$ was not affected. $A Q P 9$ could not be detected. The expression of $M R P 2$ was reduced by both concentrations of $\mathrm{As}^{\mathrm{III}}$ in testes, and the same trend was observed in duodenum and jejunum at $1000 \mathrm{ppb} \mathrm{As}^{\mathrm{III}}$. Arsenite reduced NaPiIIb expression in duodenum at $1000 \mathrm{ppb}$, and in jejunum at both concentrations. Pit 1 and Pit 2 were not affected in any of the tissues studied, except for a tendency to decrease at $1000 \mathrm{ppb}$ in jejunum. In kidney, NaPiIIa followed the same trend as NaPiIIb, with lesser expression in arsenite-treated rats.

To assess if the reduced gene expression of phosphate transporters was reflected in the actual phosphate transport, uptake assays were performed in brush border membrane vesicles of kidney and jejunum. While no effects were observed in kidney, the net phosphate transport in jejunum was reduced by 40 and $50 \%$ in rats treated with $10 \mathrm{ppb}$ and $1000 \mathrm{ppb}$ arsenite, respectively. Plasma PTH was increased at $1000 \mathrm{ppb} \mathrm{As}{ }^{\mathrm{III}}$, and FGF23 was not modified. However, further endocrine disruption cannot be discarded. In conclusion, exposure to arsenical species causes specific changes in gene expression that can explain some of the detrimental effects of the metalloid.
Arsenite, AQP, AS3MT, MRP2, phosphate transporters, rat

P3-19

ROLE OF HEAT SHOCK PROTEIN ON HEAT STRESS RESISTANCE OF HUMAN MONOCYTIC LEUKEMIA U937 CELLS

Ana María Marchena López, Ignacio Bejarano Hernando, Javier Espino Palma, Ana Beatriz Rodríguez Moratinos, José Antonio Pariente Llanos

Universidad de Extremadura, Badajoz, Spain

Background: Heat Shock Proteins (HSP) is a superfamily of highly conserved proteins with a cytoprotective role. Under physiological conditions HSP act as chaperones however, many different cell death stimuli are well-known as inducers of HSP expression. HSP have essential anti-apoptotic properties because can block both, the intrinsic and the extrinsic apoptotic pathways at different levels. In many kinds of tumor cells, the HSP overexpression is essential for their well-known resistance to apoptosis. Material \& Method: Exponential growing U937 cells were incubated $1 \mathrm{~h}$ at $42{ }^{\circ} \mathrm{C}$ and a time course of viability (MTT), caspase-3 and -9 activities by the cleavage of specific fluorogenic substrates were studied. To evaluate the prosurvival role of HSP in U937 cell line $100 \mathrm{nM}$ Geldanamycin (GA) and $100 \mathrm{nM}$ 17-N-allylamino-17demothoxygeldanamycin (17-AAG), HSP90 inhibitors, were preincubated for $24 \mathrm{~h} ; 1 \mu \mathrm{M}$ pifithrin- $\mu$ (PFT- $\mu$ ), HSP70 inhibitor, was preincubated for $48 \mathrm{~h}$. Results: Heat shock induced an evident drop in the U937 cells viability, just after the shock. During the recovery period the viability slightly decreased in a time dependent manner. When U937 cells were heat stressed in presence of HSP inhibitors, (GA, 17-AAG and PFT- $\mu$ ) the cell viability decreases drastically with respect to those which contained functional HSP.

Conclusion: Hsp 70 and 90 proteins play an essential role in cell protection against heat stress. Inhibition of Hsp 70 and Hsp 90 proteins makes tumor cells sensitive to induced heat stress leading them to apoptotic process. These results suggest HSP as a potential target to beat acute monocytic leukemias.

Supported by Junta de Extremadura-Fondo Social Europeo de Desarrollo Regional (FEDER) grants IB13072 and GR15051. AM Marchena was beneficiary of a grant by Fundación Tatiana Pérez De Guzmán El Bueno. J. Espino holds a research postdoctoral fellowship from Junta de Extremadura (jointly financed by the European Regional Development Fund (ERDF); ref. PO14011).

U937, HSP, caspase, cell viavility, HSP inhibitor

P3-20

MELATONIN INCREASES SORAFENIB SENSITIVITY IN HEP3B LIVER CANCER CELLS THROUGH MITOPHAGY INDUCTION

Raquel Ordóñez Fernández ${ }^{(1)}$, Anna Fernández Fernández ${ }^{(1)}$, Nestor Prieto-Dominguez ${ }^{(1)}$, Carolina Mendez-Blanco ${ }^{(1)}$, Andrés 
García-Palomo ${ }^{(2)}$, Anna Baulies Domènech ${ }^{(3)}$, Carmen GarcíaRuiz $^{(3)}$, José Carlos Fernández-Checa Torres ${ }^{(3)}$, José Luis Mauriz Gutiérrez $^{(1)}$, Javier González-Gallego ${ }^{(1)}$

(1) Instituto de Biomedicina (IBIOMED), Universidad de León and Centro de Investigación Biomédica en Red de Enfermedades Hepáticas y Digestivas (CIBERehd), Instituto de Salud Carlos III, Madrid, Leon, Spain; ${ }^{(2)}$ Servicio de Oncología Médica. Complejo Asistencial Universitario de León. Hospital de León, Leon, Spain; ${ }^{(3)}$ Department of Cell Death and Proliferation, IIBBCSIC, Liver Unit-Hospital Clinic-IDIBAPS, Barcelona and Centro de Investigación Biomédica en Red de Enfermedades Hepáticas y Digestivas (CIBERehd), Instituto de Salud Carlos III, Madrid, Barcelona, Spain

Background and aims: Hepatocellular carcinoma (HCC) is the fifth most common neoplasm and the third cause of cancer death. Sorafenib (sfb) is the only available non-curative approach that has shown improvement in survival rate in advanced HCC; however, sorafenib efficacy is frequently transient, and the activation of compensatory pathways in response to drug administration leads to tumor resistance. Mitophagy, promotes turnover of damaged mitochondria and numerous studies have analyzed the effects of mitophagy deficiency on tumorigenesis, revealing that inhibition of mitophagy promotes tumor progression. Melatonin has proapoptotic, anti-angiogenic and anti-invasiveness properties in HepG2 HCC cells. However, combined treatment of melatonin plus sorafenib has not been evaluated. The aim of the study was to analyze cell viability, mitochondrial biogenesis, mitophagy and apoptosis in human HCC when sorafenib and melatonin were coadministered.

Methods: melatonin and sorafenib effect on cell viability was measured by MTT and flow cytometry in three different HCC cell lines: HepG2, HuH7 y Hep3B. Mitophagy, apoptosis and mitochondrial biogenesis protein levels were evaluated by Western blot. Mitophagy was measured by confocal microscopy and mtDNA content, and its implication in cell survival was assessed by Parkin silencing. ROS production and mitochondrial transmembrane potential was analyzed by fluorescence. Results: Sorafenib and melatonin treatments decreased HepG2 and $\mathrm{HuH} 7$ cell viability in a dose-dependent manner, but only highest doses were able to reduce Hep3B viability. Combined treatment had a higher effect on cell death, being especially significant in Hep3B cells. Confocal microscopy images denoted that $2.5 \mu \mathrm{M}$ sorafenib plus $1 \mathrm{mM}$ melatonin cotreatment stimulated mitochondria and lysosome colocalization from 6 to $12 \mathrm{~h}$ in Hep3B cells, which correlates with Parkin and Pink expression enhancement, early ROS production and mitochondrial membrane depolarization necessary for mitophagy induction. mtDNA and mitochondria protein HSP60 levels were decreased by sorafenib plus melatonin treatment. Mitochondrial fusion proteins Mitofusin 2 and OPA-1 were downregulated by cotreatment while fission (analyzed by hFis-1 levels) was upregulated, denoting that mitochondrial dynamics could be implicated in mitophagy regulation. Prolonged treatments with both drugs induced an increase in apoptotic markers expression, but these increases were prevented when mitophagy was modulated through Parkin knockdown.

Conclusions: Our results suggest that combination with melatonin reduces resistance to sorafenib and enhances programmed cell death in Hep3B cells by a mechanism that could implicate mithophagy, mitochondrial fusion and fission processes
Hepatocarcinoma, melatonin, sorafenib, mitophagy, oxidative stress

P3-21

ROUTES FOR THE OLIGOMERIC FORMATION OF FUNCTIONAL KV7.1/KCNE1 CHANNELS

Anna Oliveras ${ }^{(1)}$, Clara Serrano-Novillo ${ }^{(1)}$, Irene Estadella ${ }^{(1)}$, Daniel Sastre $^{(1)}$, Núria Comes ${ }^{(2)}$, Antonio Felipe ${ }^{(1)}$

(1) Molecular Physiology Laboratory, Departament de Bioquímica i Biomedicina Molecular, Institut de Biomedicina (IBUB), Universitat de Barcelona, 08028 Barcelona, Spain; ${ }^{(2)}$ Departament de Ciències Fisiològiques I, Institut de Neurociències, Universitat de Barcelona, 08036 Barcelona, Spain

The voltage-gated potassium channel $\mathrm{K}_{\mathrm{v}} 7.1$ associates with the KCNE1 $\beta$-subunit. This oligomeric interaction generates the slowly activating delayed rectifying potassium current, $\mathrm{I}_{\mathrm{Ks}}$, which participates during the repolarization of cardiac action potential. Mutations in either subunit lead to severe cardiac channelopathies such as long QT syndrome. The functional effect of KCNE1 onto $\mathrm{K}_{\mathrm{v}} 7.1$ and the subsequent specific interaction domains have been widely described, but there is still controversy about the specific intracellular compartment where the assembly of the complex takes place. We demonstrate that $\mathrm{K}_{\mathrm{v}} 7.1-\mathrm{KCNE} 1$ complex is not built early at the initial stages of the secretory pathway, within the endoplasmic reticulum. In fact, both channel subunits can use different routes reaching the plasma membrane. Thus, the disruption of Golgi apparatus impairs $\mathrm{KCNE1}$, but not $\mathrm{K}_{\mathrm{v}} 7.1$, targeting to the cell surface. Our results indicate that $\mathrm{KCNE} 1$ relays on COPII-dependent forward trafficking machinery and $\mathrm{K}_{\mathrm{v}} 7.1$ can alternatively use a non-conventional secretory pathway. Upon the formation of the functional complex, $\mathrm{K}_{\mathrm{v}} 7.1$ redirects KCNE1 to the COPII-independent route to the membrane. Finally, studies with plasma membrane lawn preparations suggest that the oligomeric complex is fully assembled at the cell surface, thereby suggesting that the $\mathrm{K}_{\mathrm{v}} 7 \cdot 1-\mathrm{KCNE} 1$ association takes place in an alternative route late in the secretory pathway but nearby to the cell surface.

Supported by MINECO, Spain (BFU2014-54928-R and BFU2015-70067-REDC).

Kv7.1, KCNE1, Traffic, Assembly

P3-22

\section{EGF-MEDIATED ENDOCYTOSIS OF KV1.3}

Katarzyna Styrczewska ${ }^{(1)}$, Ramón Martínez-Mármol (2), Núria Comes ${ }^{(3)}$, Irene Estadella ${ }^{(1)}$, Mireia Peréz-Verdaguer ${ }^{(1)}$, Eduardo Soriano ${ }^{(4)}$, Alexander Sorkin ${ }^{(5)}$, Antonio Felipe ${ }^{(1)}$

(1) Molecular Physiology Laboratory, Departament de Bioquímica i Biomedicina Molecular, Institut de Biomedicina (IBUB), Universitat de Barcelona, Barcelona, Spain; ${ }^{(2)}$ Clem Jones Centre 
for Ageing Dementia Research, Queensland Brain Institute, The University of Queensland, Brisbane, Queensland 4072, Australia; (3) Laboraory of Neurophysiology, Institut de Neurociencia. Universitat de Barcelona, Barcelona, Spain; ${ }^{(4)}$ Departament de Biologia Celular, CIBERNED, Universitat de Barcelona, Barcelona, Spain; ${ }^{(5)}$ Department of Cell Biology and Physiology, University of Pittsburgh School of Medicine, Pittsburgh, PA 15261, United States

The voltage-dependent potassium channel Kv1.3 plays important roles in immunity, axonal growth targeting, sensory discrimination, metabolism and insulin resistance. Kv1.3 is one of the $\mathrm{Kv}$ channels whose regulation by phosphorylation has been most extensively studied. EGF, via binding to specific receptors (EGFR, ERB3), triggers important tyrosine kinase-dependent signaling which strongly down-regulates Kv1.3 currents. However, although EGF induces internalization of many membrane proteins, no evidence describes changes of Kv1.3 current amplitude as a consequence of channel endocytosis. Here we describe that Kv1.3 undergoes EGF-dependent endocytosis. Antibody-feeding internalization assay revealed that changes in Kv1.3 distribution upon EGFR activation corresponds to induced-endocytosed channels and immunocytochemistry approaches showed colocalization of intracellular Kv1.3 with endocytic vesicles markers. Mutational analysis revealed that tyrosines and other putative motive sequences where not apparently important for EGFR activationmediated internalization. However, newly identified putative sequence for ERK1/2-mediated threonine phosphorylation revealed its potential importance in surface Kv1.3 down-regulation. Knockdown of clathrin heavy chain or dynamin II by small interfering RNAs dramatically inhibited EGFR activation-mediated endocytosis of Kv1.3. By using a wide repertoire of techniques, we demonstrated that changes in Kv1.3 distribution upon EGFR activation corresponded to a massive Kv1.3 endocytosis via clathrin-coated pits mechanisms which localized the Kv1.3 channels in the lysosomal degradative pathway. This EGF mechanism is highly relevant because halted proliferation but increased neuronal migration from the subventricular zone of the forebrain to the olfactory bulb.

Supported by MINECO, Spain (BFU2014-54928-R and BFU2015-70067-REDC).

Endocytosis, Map kinases, Olfactory bulb, Sensory neurons, Tyrosine kinases, Voltage-dependent potassium channels

P3-23

STUDY OF GLYCOLIC ACID PEELING TREATMENT EFFECT AND QUERCETIN ON FIBROBLASTS

Alvaro Casanova, Dessiree Pereboom Maicas, Isabel Fernandez, Ruth Solanas, Jose Joaquin Garcia, José Octavio Alda

Universidad de Zaragoza, Zaragoza, Spain

Chemical peels have seen increased popularity in recent years as a treatment for many skin conditions with the main objective of skin rejuvenation. Chemical peels also described a variety of adverse effects. It may reduce wrinkles, acne scarring and improve many other skin pathologies. And they are also contraindicated for patients with bacterial, viral, fungal or with a history of photosensitivity.

Glycolic acid (hydroacetic acid or hydroxyacetic acid) is the smallest $\alpha$-hydroxy acid (AHA). This is the most used chemical agent in these peeling due to their ability to penetrate into the deeper skin. Typically the glycolic acid reacts with the upper layer of the epidermis, resulting in the release of dead cells.

Methods: We use human fibroblast primary cell culture with gradually increasing doses of the Glycolic acid treatment $(2.5,5$, $10,50,100,150$ y $300 \mathrm{mM}$ ).

The glycolic treatment solutions were previously buffered with Tris base for remove $\mathrm{pH}$ effect.

Quercetin flavonoid is an excellent free radicals scavenger, this research is quantifying the possible protective effects against the damage produced by Glycolic acid treatment.

Vitality levels and the different cells deaths determinations were performed by image cytometry (ImageStream X, Amnis) with Annexin V-IP Kit and MTT Test.

Results: DL ${ }_{50}$ was determined of $50-100 \mathrm{mM}$ for Glycolic acid treatment. The cytotoxic effect was shown to be dose-dependent increasing cell death. Very low doses of quercetin $(2.5 \mu \mathrm{M})$ are able to reduce the damage produced by $50 \mathrm{mM}$ Glycolic acid treatment in approximately $60 \%$.

The most part of the Glycolic acid damage was observed in late apoptosis with five times higher than controls cells. Quercetin proved able to reduce the damage of Glycolic acid through a reduction of the late apoptosis in approximately $50 \%$.

Conclusion: Quercetin decreases early and late apoptosis in damage by glycolic acid, an effect that could be explained by senolytic and rejuvenating properties of this drug.

Peeling, skin, Glycolic acid, Quercetin, MTT and image cytometry

P3-24

LETHAL EFFECTS OF SULFUR MUSTARD ON FIBROBLAST POPULATIONS

Alvaro Casanova Flor De Lis, Ana Muñoz, Yaiza Marzo, Jose Octavio Alda, Desiree Pereboom

Universidad de Zaragoza, Zaragoza, Spain

Sulfur mustard, commonly known as mustard gas, is a synthetic toxic agent which was used over the last century with warfare means. It is a vesicant substance due to its skin absorption producing blisters, edema, irritation, ulceration and burns. It can also be absorbed through ocular (conjunctiva) and respiratory tract. The main mechanism of action is alkylation of several cellular components, including the DNA. Its intervention in inflammatory mechanisms produces the liberation of reactive oxygen species that cause DNA mutations, altering the cellular division and finally cellular death. 
Previous studies show that quercetin can block the harmful effects of free radicals, decreasing oxidative stress. Therefore our objective is studying the possible protective effects of quercetin against mustard gas.

Materials and methods: We have used human fibroblast primary cell cultures with gradually increasing doses of sulfur mustard exposure $(200,500,1000$ and $2000 \mu \mathrm{M})$. We determined the $\mathrm{LD}_{50}$ (lethal dose $50 \%$ ) and used different quercetin doses to determine the possible protection of this antioxidant agent against mustard gas.

Vitality and mortality (early apoptosis, late apoptosis, necrosis and necroptosis) were analyzed by image cytometry (Amnis ImageStream X) with two fluorochromes, Annexin V-Propidium Iodide and colorimetric assay MTT (Thiazolyl Blue Tetrazolium) test.

Results: In this study fibroblasts were exposed to increasing concentrations of sulfur mustard gas (200-2000 $\mu \mathrm{M})$ and compared to untreated controls.

Mustard gas $\mathrm{LD}_{50}$ was determined at $1290 \mu \mathrm{M}$ exposure. The cytotoxic effect turned out to be dose-dependent. Low doses of quercetin $(0.5$ and $2.5 \mu \mathrm{M})$ reduced cell death produced by $1000 \mu \mathrm{M}$ mustard gas in $66.22 \pm 2.88 \%$ and $88.96 \pm 2.22 \%$ respectively. The worst damage of mustard gas was observed in late apoptotic and necroptotic cells. Late apoptosis was quadruplicated (from $6.697 \pm 0.860 \%$ to $25.383 \pm 1.787 \%$ ) and necroptosis (from $1.867 \pm 0.362 \%$ to $12.779 \pm 0.388 \%$ ) was sextuplicated compared to control cells.

Conclusion: Late apoptosis after mustard gas exposure was quadruplicated, whereas necroptosis was sextuplicated compared to control cells.

For the first time it has been confirmed the protective effect of the quercetin against cellular toxicity caused by sulfur mustard. This study has used quercetin concentrations of 0,5 and $2,5 \mu \mathrm{M}$ in human fibroblasts exposed to sulfur mustard at $1000 \mu \mathrm{M}$, obtaining a cellular protection of 60 and $90 \%$ respectively.

Sulfur mustard, Quercetin, Apoptosis, MTT, Image cytometry

P3-25

\section{UV RADIATIONS EFFECTS ON FIBROBLASTS}

Alvaro Casanova Flor De Lis, Desiree Pereboom, Yaiza Marzo Jose Octavio Alda

Universidad de Zaragoza, Zaragoza, Spain

Ultraviolet radiation (UV) belongs to the range of the electromagnetic spectrum and extends from the violet of the visible spectrum to X-ray area.

This radiation has produced a lot of beneficial effects to the skin such as vitamin D synthesis or production of endorphins, but also has some harmful effects. UV radiation causes apoptosis and necrosis in cellular models, in addition to causing human skin redness (erythema) and ocular inflammation (keratitis, conjunctivitis).
The objective of this study is to quantify the effect of UV radiation on mortality/vitality and oxidative stress production of the primary culture fibroblasts.

Methods: Fibroblasts were irradiated with ultraviolet light. So for UVB radiation it was achieved with a lamp Philips PL-L 36 W/01/ $4 \mathrm{P}$ with a bandwidth between 305 and $315 \mathrm{~nm}$. with a peak at $311 \mathrm{~nm}$. For UVC irradiations use a tube Philips TUV30W / G30 (Witt Lighting Co., Ltd., Guangdong, China) with a peak at $253.7 \mathrm{~nm}$.

So much for UVB and UVC will make the determination of oxidative stress (superoxide anion, hydrogen peroxide and singlet oxygen) and the different cells deaths determinations were performed by image cytometry (ImageStream X, Amnis) with $\mathrm{cFDA}($ Carboxyfluerescein diacetate)/IP(Propidium iodide), HE (Hidroetidine), DHR123(Dihydrorhodamine 123), ADPA (Anthracenedipropionic acid) and Annexin V-IP Kit.

Results: The effect of UV radiation on fibroblasts is dose dependent for UVB and for UVC. UVB radiation to get an $\mathrm{LD}_{50}$ (Lethal dose $50 \%$ ) of $6.97 \mathrm{~J} / \mathrm{cm}^{2}$ and for $\mathrm{UVC}^{\mathrm{L}} \mathrm{LD}_{50}$ is $1,269 \mathrm{~J} / \mathrm{cm}^{2}$.

Between the mechanisms of action of UV radiation is important to the genesis of oxygen free radicals. UVB and UVC increased superoxide anion, singlet oxygen and hydrogen peroxide; the effects of UVC were 30 to 50 times more potent than UVB. Moreover, UV radiation increases cell death rates of all study populations compared to the control (early apoptotic (21,4 \% UVB and $5.23 \%$ UVC), necrotic $(1,75 \%$ UVB and $4.87 \%$ UVC), late apoptotic(4,68\% UVB and $16.97 \%$ UVC) and necroptotic ( $8,7 \%$ UVB and $6.58 \%$ UVB)). For UVB radiation the highest growth is found in the early apoptotic population, for UVC is for late apoptotic population.

Conclusion: UVB and UVC radiation increases cell death on primary fibroblasts culture, mainly through apoptosis mechanism possibly caused by increased production of free radicals.

Ultraviolet radiation, skin, oxidative stress, apoptosis and image cytometry

\section{P3-26}

STUDY OF THE PREVALENCE OF THYROID DISORDERS IN PEOPLE LIVING IN ARAGON WITH PREMUTATION IN THE FMR1 GENE

Noelia Cuadrado ${ }^{(1)}$, Silvia Izquierdo ${ }^{(2)}$, José Ignacio González ${ }^{(2)}$, José Puzo ${ }^{(2)}$, Manuel Guerra ${ }^{(3)}$

(1) Blackhills Diagnostic Resources, Zaragoza, Spain; ${ }^{(2)}$ Hospital Universitario Miguel Servet, Zaragoza, Spain; ${ }^{(3)}$ Facultad de Medicina, Universidad de Zaragoza, Zaragoza, Spain

Introduction: For years existed the belief that premutation carriers [from 55 to 200 repeats of the cytosine-guanine-guanine (CGG) triplet] in the FMR1 gene showed no clinical manifestation. Associated alterations currently accepted and based on scientific studies are: emotional and psychological problems, premature ovarian failure associated with fragile X syndrome (FXPOI) and 
tremor-ataxia syndrome associated with fragile $\mathrm{X}$ syndrome (FXTAS). Thyroid problems have been described to occur more often among individuals with premutation than what would be expected in the general population. But this association of the premutation with the thyroid disorder is still poorly established. Thyroid hormones have an intracellular receptor and act on the nuclear DNA. FMRP, encoded by the FMR1 gene, is an intracellular protein that may be located at three sites: 1 . In the nucleus helps DNA transcription; 2. In the cytoplasm helps translation of mRNA to ribosomes and its transcription; and 3. In the membranes interacts with neurotransmitters and growth factors and promotes the development of microtubules.

Objective: To study the prevalence of alterations in thyroid hormones in patients with premutation in the FMR1 gene in population resident in Aragon.

Materials and methods: The clinical records of 11 patients genetically diagnosed of their condition as FMR1 premutated by Triplet Repeat Primed-PCR (TP-PCR) in DNA samples from peripheral blood were reviewed. The profile of thyroid hormones in these 11 patients was evaluated.

Results: In 3 of them (27.27\%) alterations compared to normal levels were found: 2 of them $(18.18 \%)$ presented hypothyroidism, and 1 (9.09\%) had T3 elevated keeping T4 and TSH in the normal range.

Conclusion: This last patient could present a subclinical hyperthyroidism worthy of being studied. This makes it necessary to consider a protocol for requesting thyroid function test in all patients genetically diagnosed as premutation carriers in the FMR1 gene, presenting a number of CGG triplet repeats between 55 and 200. It would be recommendable to assess in these individuals the presence of attention deficit disorder and hyperactivity in both adult and pediatric age patients.

FMR1, Premutation, Trinucleotide CGG, Repeat Expansion, Hyperthyroidism, Thyroid Hormones

\section{P3-27}

\section{STUDY OF QUERCETIN ON ULTRAVIOLET-IRRADIATED FIBROBLASTS}

Alvaro Casanova, Desiree Pereboom, Yaiza Marzo Jose Octavio Alda

Universidad de Zaragoza, Zaragoza, Spain

Summary: Quercetin is a typical flavonoid can also be present in high concentrations as a secondary metabolite in fruits and vegetables. Quercetin can also be present in some manufactured products such as red wine and olive oil. One of its main advantages is that flavonoids can regenerate other antioxidants by donating its hydrogen free radical. The antioxidant capacity of flavonoids due to the high number of hydroxyl substituents in each molecule of flavonoid, wich provides direct effect on the hydrogen donnathing ability and sweeper free radical.

Bearing in mind UV radiation increases cell death, especially by free radical liberation and that quercetin is an excellent scavenger, this study proposes to quantify the possible protective effect of quercetin on irradiated cells.
Methods: We use primary human fibroblast cell culture model exposed to UV radiation and we analize the different damages depending on previus quercetin treatments. We expect a reduction in the radiations damage exposed to we are going to measure the effects evaluating viability/mortality, oxidative stress and membrane potential and we expect due to quercetin pretreatment.

We using the morphological characteristics of image cytometry (Image Stream X, Amnis) With Annexin V-IP Kit by we will be able to differentiate five types ofcellular populations (live, early apoptotic, necrotic, late apoptotic and necroptotic).

Results: Treatment with quercetin produced reductions in mortality and increased vitality of all samples irradiated with UVB and UVC. At a concentration of $1 \mathrm{mM}$ quercetin treatment reduces $60 \%$ of UVB radiation damage and $25 \%$ UVC damage. Also in fibroblasts subjected to pretreatment with quercetin decreased superoxide anion and singlet oxygen production up to $90 \%$, with $100 \%$ protection to hydrogen peroxide.

Quercetin reduces Reducers population of five study Populations against irradiated fibroblasts. The greatest reduction effect occurs in populations that more increase with UV radiation, early apoptosis for UVB and late apoptosis for UVC.

Conclusion: Quercetin is capable of reducing the increase mortality and oxidative stress produced by UV radiation, mainly reducing the production of apoptotic mechanism. These results show quercetin that would be reliable to selectively induce death only senescent and damaged cells.

Ultraviolet radiation, skin, oxidative stress, apoptosis, quercetin and image cytometry

\section{P3-28}

FUNCIONAL CHARACTERIZATION OF 11 MISSENSE MUTATIONS ASSOCIATED WITH HUMAN MITOCHONDRIAL HMG-COA SYNTHASE DEFICIENCY

María Hernández-Marcos ${ }^{(1)}$, M a Esperanza Teresa-Rodrigo ${ }^{(1)}$, Sheila López-Triguero ${ }^{(1)}$, Sergio Gil-Clavero ${ }^{(1)}$, Francisco Javier Santiago-Arcos ${ }^{(1)}$, Jorge García Ortín ${ }^{(1)}$, Paulino Gómez-Puertas ${ }^{(2)}$, Beatriz Puisac-Uriol ${ }^{(1)}$, Juan Pié-Juste ${ }^{(1)}$

(1) Unit of Clinical Genetics and Functional Genomics, Departments of Pharmacology-Physiology and Paediatrics, School of Medicine, University of Zaragoza, CIBERER-GCV and IIS-Aragon, Zaragoza, Spain; ${ }^{(2)}$ Center of Molecular Biology "SeveroOchoa” CSIC-UAM, Madrid, Spain

The human mitochondrial 3-hydroxy-3-methylglutaryl-CoA (HMG-CoA) synthase deficiency is a rare autosomal recessive metabolic disorder caused by mutations in the HMGCS2 gene. This illness affects ketone-body synthesis inside the mitochondria, and can be critical in hypoglycemic conditions, when the absence of an alternative fuel may evolve to hypoglycemic coma. Early diagnosis is essential because most symptoms can be reverted after glucose administration.

Hither to, the enzymatic assay in liver cells from the patient was the method of choice to confirm the diagnosis, since there are no specific clinical or biochemical markers. However, the need for performing a hepatic biopsy and the interference with the cytosolic isoform, complicated the diagnosis. 
Our group developed an in vitro enzymatic assay to demonstrate the pathogenicity of the mutations, thus avoiding the hepatic biopsy and the interference with the cytosolic enzyme. In a previous work we proved this method on 8 mutations, and here we extend the study to all known mutations, including four reported here for the first time.

Objectives: To confirm the diagnostic validity of the in vitro method to study mutations in the HMGCS2 gene through the analysis of 11 missense mutations. Moreover, the impact of each mutation on enzymatic activity will be evaluated with a 3D model. Methods: Functional studies were carried out on eleven mutations of the HMGCS2 gene found in seven patients, using the new method of measurement of enzyme activity. The impact of each mutation on protein structure was analyzed with a $3 \mathrm{D}$ bioinformatic model.

Results: All the recombinant proteins were overexpressed. Total absence of enzymatic activity was observed in eight of them (p.R122W, p.M146R, p.G169D, p.W185R, p.G232V, p.L266S, p.I407T and p.R501Q), while three of them showed some activity: p.V144L and p.Y503C about $8 \%$, and p.R505Q about $70 \%$ compared to the wild-type protein. Bioinformatic predictions correlated with the experimental observations, except for the mutation p.R505Q located at the carboxyl terminal end. In addition, four new missense mutations in the HMGCS2 gene are reported for the first time; c.334C $>$ T (p.R112W), c.430G $>$ T (p.V144L), c. $437 \mathrm{~T}>\mathrm{G}$ (p.M146R) and c.1502G $>$ A (p.R501Q).

Conclusion: These results confirm the reliability of the new method to analyze mutations in the HMGCS2 gene. Furthermore, the finding of an allelic variant with high activity (70 \%), and two variants with $8 \%$ of activity, demonstrates that total depletion of enzymatic activity is not essential for the disease. Additionally, a fine correlation between the experimental results and the 3D model predictions is established.

3-hydroxy-3-methylglutaryl-CoA synthase deficiency, HMGCS2 gene, enzymatic activity

P3-29

CHARACTERIZATION OF THE EXPRESSION PATTERNS OF ISOFORMS A AND B OF THE NIPBL GENE IN FETAL AND ADULT HUMAN TISSUES: EXPLANATION FOR UNUSUAL MILD PHENOTYPE

$\mathrm{M}^{\mathrm{a}}$ Esperanza Teresa-Rodrigo ${ }^{(1)}$, María Hernández-Marcos ${ }^{(1)}$, Beatriz Puisac Uriol ${ }^{(1)}$, M ${ }^{\mathrm{a}}$ Concepción Gil-Rodríguez ${ }^{(1)}$, Carolina Baquero-Montoya ${ }^{(2)}$, Inés Bueno Martínez ${ }^{(3)}$, Sheila LópezTriguero ${ }^{(1)}$, Sergio Gil-Clavero ${ }^{(1)}$, Francisco Javier SantiagoArcos ${ }^{(1)}$, Jorge García Ortín ${ }^{(1)}$, Feliciano J. Ramos Fuentes ${ }^{(3)}$, Juan Pié Juste ${ }^{(1)}$

(1) Unit of Clinical Genetics and Functional Genomics, Departments of Pharmacology-Physiology and Paediatrics, School of Medicine, University of Zaragoza, CIBERER-GCV and IIS-Aragon, Zaragoza, Spain; ${ }^{(2)}$ Unit of Clinical Genetics and Functional
Genomics, Departments of Pharmacology-Physiology and Paediatrics, School of Medicine, University of Zaragoza, CIBERERGCV and IIS-Aragon, Zaragoza, Spain. Department of Paediatrics, Hospital Pablo Tobón Uribe, Medellín, Colombia; ${ }^{(3)}$ Unit of Clinical Genetics and Functional Genomics, Departments of Pharmacology-Physiology and Paediatrics, School of Medicine, University of Zaragoza, CIBERER-GCV and IIS-Aragon, Zaragoza, Spain. Clinical Genetics Unit, Service of Paediatrics, Hospital "Lozano Blesa" Medical School, University of Zaragoza, CIBERER-GCV and IIS-Aragón, Zaragoza, Spain

Background: Cornelia de Lange syndrome (CdLS) is a congenital developmental disorder characterized by craniofacial dysmorphia, growth retardation, limb malformations and intellectual disability. Approximately $60 \%$ of patients have pathological variants in the $N I P B L$ gene. Although several physiological splicing variants of $N I P B L$ have been detected, the main and largest ones are isoforms $\mathrm{A}$ and $\mathrm{B}$, which only differ in their C-terminal end. We have performed the first evaluation of the expression patterns of these variants in fetal and adult tissues. The results obtained support the atypical mild CdLS phenotype observed in two brothers carrying a mutation in NIPBL that only affects to the isoform A.

Objective: To know the expression patterns of the main physiological splicing variants of $N I P B L$ in fetal and adult tissues. Additionally, we have used this data to explain the mild phenotype observed in two brothers with a mutation in NIPBL.

Material and methods: Expression patterns of the isoforms A and $\mathrm{B}$ were evaluated on the most relevant fetal and adult tissues obtained from commercial cDNA panels. Genomic DNA of the patients was isolated from peripheral blood leukocytes, fibroblasts, oral mucosa epithelial cells and urine epithelial cells. Pyrosequencing analyses were performed to evaluate the relative amount of each allele.

Results: Isoforms A and B show a similar pattern of distribution across all the tissues, though the expression of the isoform A looks slightly higher in all of them. The expression of both isoforms is very homogenous in fetal tissues, but big differences can be observed among adult tissues. In particular, adult liver, pancreas, placenta and heart show the highest level of expression; followed by lung and kidney. The weakest expression appears in brain and skeletal muscle, with no presence of isoform $\mathrm{B}$ in this last tissue. The mutation c.8387A $>$ G, p.Y2796C found in two brothers with atypical mild CdLS phenotype, is located at the end of the NIPBL gene, and it only affects to the isoform A. Pyrosequencing studies of different tissues of both patients ruled out the presence of somatic mosaicism.

Conclusions: Isoforms A and B are expressed in all the tissues evaluated, except for isoform B on skeletal muscle. They show a similar pattern, homogeneous in fetal tissues and very heterogeneous in adult tissues. The coexistence of both isoforms supports that the location of the variant $c .8387 \mathrm{~A}>\mathrm{G}$, that only alters the isoform A, is likely to be the cause of the atypical mild phenotype observed in the patients.

Cornelia de Lange syndrome (CdLS), NIPBL gene, expression patterns, physiological splicing variants, isoform A, isoform B. 